\title{
X-ray spectral variability of Seyfert 2 galaxies ${ }^{\star}$
}

\author{
L. Hernández-García ${ }^{1}$, J. Masegosa ${ }^{1}$, O. González-Martín² ${ }^{2}$ and I. Márquez ${ }^{1}$ \\ 1 Instituto de Astrofísica de Andalucía, CSIC, Glorieta de la Astronomía, s/n, 18008 Granada, Spain \\ e-mail: lorena@iaa.es \\ 2 Centro de radioastronomía y Astrofísica (CRyA-UNAM), 3-72 (Xangari), 8701 Morelia, Mexico
}

Received 18 March 2015 / Accepted 2 May 2015

\begin{abstract}
Context. Variability across the electromagnetic spectrum is a property of active galactic nuclei (AGN) that can help constrain the physical properties of these galaxies. Nonetheless, the way in which the changes happen and whether they occur in the same way in every AGN are still open questions.

Aims. This is the third in a series of papers with the aim of studying the X-ray variability of different families of AGN. The main purpose of this work is to investigate the variability pattern(s) in a sample of optically selected Seyfert 2 galaxies.

Methods. We use the 26 Seyfert $2 \mathrm{~s}$ in the Véron-Cetty and Véron catalog with data available from Chandra and/or XMM-Newton public archives at different epochs, with timescales ranging from a few hours to years. All the spectra of the same source were simultaneously fitted, and we let different parameters vary in the model. Whenever possible, short-term variations from the analysis of the light curves and/or long-term UV flux variations were studied. We divided the sample into Compton-thick and Compton-thin candidates to account for the degree of obscuration. When transitions between Compton-thick and thin were obtained for different observations of the same source, we classified it as a changing-look candidate.

Results. Short-term variability at X-rays was studied in ten cases, but variations are not found. From the 25 analyzed sources, 11 show long-term variations. Eight (out of 11) are Compton-thin, one (out of 12) is Compton-thick, and the two changing-look candidates are also variable. The main driver for the X-ray changes is related to the nuclear power (nine cases), while variations at soft energies or related to absorbers at hard X-rays are less common, and in many cases these variations are accompanied by variations in the nuclear continuum. At UV frequencies, only NGC 5194 (out of six sources) is variable, but the changes are not related to the nucleus. We report two changing-look candidates, MARK 273 and NGC 7319.

Conclusions. A constant reflection component located far away from the nucleus plus a variable nuclear continuum are able to explain most of our results. Within this scenario, the Compton-thick candidates are dominated by reflection, which suppresses their continuum, making them seem fainter, and they do not show variations (except MARK 3), while the Compton-thin and changing-look candidates do.
\end{abstract}

Key words. galaxies: active - X-rays: galaxies - ultraviolet: galaxies

\section{Introduction}

It is widely accepted that active galactic nuclei (AGN) are powered by accretion onto a supermassive black hole (SMBH, Rees 1984). Among them, the different classes of Seyfert galaxies (type 1/type 2) have led to postulating a unified model (UM) for all AGN (Antonucci 1993; Urry \& Padovani 1995). Under this scheme, the SMBH is fed by the accretion disk that is surrounded by a dusty torus. This structure is responsible for obscuring the region where the broad lines are produced (known as broad line region, BLR) in type 2 objects, while the region where the narrow lines are produced (narrow line region, NLR) is still observed at optical frequencies. The difference between type 1 and 2 objects is therefore due to orientation effects.

In agreement with the UM, the type 1/type 2 classifications at X-ray frequencies are based on the absorption column density, $N_{\mathrm{H}}$, because it is related with the obscuring material along our line of sight (Maiolino et al. 1998); therefore, we observe a type 1 Seyfert if $N_{\mathrm{H}}<10^{22} \mathrm{~cm}^{-2}$, i.e., unobscured view of the inner parts of the AGN, and a type 2 if the column density is higher, i.e., obscured view through the torus (e.g., Risaliti et al. 2002).

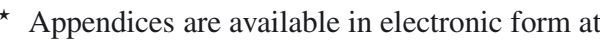
http: //www . aanda.org
}

When $N_{\mathrm{H}}>1.5 \times 10^{24} \mathrm{~cm}^{-2}$, the absorbing column density is higher than the inverse of the Compton-scattering cross-section, and the sources are known as Compton-thick (Maiolino et al. 1998).

In fact, X-rays are a suitable tool for studying AGN because they are produced very close to the SMBH and because of the much smaller effect of obscuration at these frequencies than at UV, optical, or near-IR. Numerous studies have been made at $\mathrm{X}$-ray frequencies to characterize the spectra of Seyfert galaxies (e.g., Turner et al. 1997; Risaliti 2002; Guainazzi et al. 2005b,a; Panessa et al. 2006; Cappi et al. 2006; Noguchi et al. 2009; LaMassa et al. 2011; Brightman \& Nandra 2011a). The present work is focused on Seyfert 2 galaxies, which represent $\sim 80 \%$ of all AGN (Maiolino \& Rieke 1995). The works mentioned above have shown that the spectra of these objects are characterized by a primary power-law continuum with a photoelectric cut-off, a thermal component, a reflected component, and an iron emission line at $6.4 \mathrm{keV}$. It is important to appropiately account for the physical parameters of their spectra in order to constrain physical properties of the nuclei.

Given that variability across the electromagnetic spectrum is a property of all AGN, understanding these variations offers an exceptional opportunity to constrain the physical characteristics 
of AGN, which are known to show variations on timescales ranging from a few days to years (Peterson 1997). The first systematic variability study of Seyfert 2 galaxies was performed by Turner et al. (1997) using ASCA data. Their results show that short-term variability (from hours to days) is not common in Seyfert 2s, in contrast to what is observed in Seyfert 1 (e.g., Nandra et al. 1997). Because these galaxies are obscured by the torus, the lack of variations could come from these sources being reflection-dominated, as shown by some authors that studied Compton-thick sources (Awaki et al. 1991; LaMassa et al. 2011; Matt et al. 2013; Arévalo et al. 2014). However, a number of Seyfert 2 s actually do show variations. The study of the variability has been approached in different ways from the analysis of the light curves to study of short-term variations (Awaki et al. 2006), through count-rate or flux variations (Isobe et al. 2005; Trippe et al. 2011), or comparisons of spectra of the same source at different epochs (LaMassa et al. 2011; Marinucci et al. 2013; Marchese et al. 2014). The observed variations may be related with absorbing material that crosses our line of sight (Risaliti et al. 2002, 2010) and/or can be intrinsic to the sources (Evans et al. 2005; Sobolewska \& Papadakis 2009; Braito et al. 2013). A few Seyfert 2s also showed changes from being reflectiondominated to transmission-dominated objects, so were called changing-look objects (Guainazzi et al. 2002; Guainazzi 2002; Matt et al. 2003; Risaliti et al. 2010).

Although it is well established that a number of Seyfert $2 \mathrm{~s}$ are variable, it is unknown whether the same kind of variation is common for all the nuclei or, more important, what drives those variations. It is the purpose of this paper to systematically study the variability pattern in X-rays in Seyfert 2 nuclei. This is the third in a series of papers aimed at studying the X-ray variability in different families of AGN. In Hernández-García et al. $(2013,2014)$, this study was made for LINERs, while the study of Seyfert 1 and the comparison between different families of AGN will be presented in forthcoming papers.

This paper is organized as follows. In Sect. 2 the sample and the data are presented, and data reduction is explained in Sect. 3. The methodology used for the analysis is described in Sect. 4, including individual and simultaneous spectral fittings, comparisons using data with different instruments, longterm X-ray and UV variations, short-term X-ray variations, and Compton-thickness analysis. The results derived from this work are explained in Sect. 5 and are discussed in Sect. 6. Finally, the main conclusions are summarized in Sect. 7.

\section{Sample and data}

We used the 13th edition of the Véron-Cetty and Véron catalog (Véron-Cetty \& Véron 2010), which contains quasars and active galactic nuclei. We selected galaxies located at redshift below 0.05 and classified as Seyfert 2 (S2) or objects with broad polarized Balmer lines detected (S1h). Indeed, S1h objects are those optically classified as Seyfert 2 that show broad lines in polarized light, which is the reason for their selection. This subsample includes $730 \mathrm{~S} 2$ and $27 \mathrm{~S} 1 \mathrm{~h}$.

We searched for all the publicly available data for sources with observations in more than one epoch with Chandra and/or XMM-Newton using the HEASARC ${ }^{1}$ browser up to May 2014. This first selection includes 73 nuclei. To be able to properly fit and compare spectra at different epochs, we selected sources with a minimum of 400 number counts in the $0.5-10.0 \mathrm{keV}$ energy band, as required to use the $\chi^{2}$-statistics. Thirty-four

\footnotetext{
1 http://heasarc.gsfc.nasa.gov/
}

galaxies and nine observations did not met this criterium and were excluded from the sample. Objects affected by a pileup fraction higher than $10 \%$ were also removed, which made us exclude three objects and 14 observations.

For the remaining 36 nuclei we searched for their optical classifications in the literature with the aim of including only pure Seyfert 2 objects in the sample. Nine galaxies were excluded following this condition: NGC 4258, and NGC 4374 (S1.9 and L2 in Ho et al. 1997), 3C 317.0 and 3C 353.0 (LINERs in NED ${ }^{2}$ ), NGC 7314 (S1.9 in Liu \& Bregman 2005), MCG-03.34.064 (S1.8 in Aguero et al. 1994), NGC 5252 (S1.9 in Osterbrock \& Martel 1993), and NGC 835 and NGC 6251 (LINERs in González-Martín et al. 2009b). NGC 4472 was also excluded because its classification is based on the upper limits of line intensity ratios (Ho et al. 1997), and other classifications have been found in the literature (e.g., Boisson et al. 2004).

The final sample of Seyfert 2 galaxies contains 26 objects, 18 classified as S2 and 8 classified as S1h in Véron-Cetty \& Véron (2010). However, we revisited the literature to search for hidden broad-line-region (HBLR, an usual name for S1h) and non-hidden broad-line-region (NHBLR) objects (e.g., Tran et al. 1992; Tran 1995; Moran et al. 2000; Lumsden et al. 2001; Gu $\&$ Huang 2002). We found two additional HBLR (MARK 1210 and MARK 477) and five NHBLR (MARK 1066, NGC 3079, NGC 5194, NGC 5643, and NGC 7172) sources. We did not find information about the remaining 11 nuclei, so we assumed they are most probably not observed in polarized light.

The final sample of Seyfert $2 \mathrm{~s}$ in our work thus contains 26 objects (including 10 HBLR and five NHBLR). The target galaxies and their properties are presented in Table 1. Tables are in Appendix A, and notes on the individual nuclei in Appendix B and images at different wavelenths in Appendix C.1.

\section{Data reduction}

\subsection{Chandra data}

Chandra observations were obtained from the ACIS instrument (Garmire et al. 2003). Data reduction and analysis were carried out in a systematic, uniform way using CXC Chandra Interactive Analysis of Observations $\left(\mathrm{CIAO}^{3}\right)$, version 4.3. Level 2 event data were extracted by using the task ACIS-PROCESS-EVENTS. Background flares were cleaned using the task LC_CLEAN.SL ${ }^{4}$, which calculates a mean rate from which it deduces a minimum and maximum valid count rate and creates a file with the periods that are considered by the algorithm to be good.

Nuclear spectra were extracted from a circular region centered on the positions given by $\mathrm{NED}^{5}$. We chose circular radii, aiming to include all possible photons, while excluding other sources or background effects. The radii are in the range between 2-5" (or 4-10 pixels, see Table A.1). The background was extracted from circular regions in the same chip that are free of sources and close to the object.

For the source and background spectral extractions, the DMEXTRACT task was used. The response matrix file (RMF) and ancillary reference file (ARF) were generated for each source region using the MKACISRMF and MKWARF tasks, respectively. Finally, the spectra were binned to have a minimum of 20 counts per spectral bin using the GRPPHA task (included in FTOOLS), to be able to use the $\chi^{2}$ statistics.

\footnotetext{
http://ned.ipac.caltech.edu/

http://cxc.harvard. edu/ciao4.4/

4 http://cxc.harvard.edu/ciao/ahelp/lc_clean.html

5 http://ned.ipac.caltech.edu/
} 
Table 1. General properties of the sample galaxies.

\begin{tabular}{|c|c|c|c|c|c|c|c|c|}
\hline $\begin{array}{l}\text { Name } \\
\text { (1) }\end{array}$ & $\begin{array}{c}\text { RA } \\
(\mathrm{J} 2000) \\
(2)\end{array}$ & $\begin{array}{c}\text { Dec } \\
(\mathrm{J} 2000) \\
(3)\end{array}$ & $\begin{array}{c}\text { Dist. }^{1} \\
(\mathrm{Mpc}) \\
(4) \\
\end{array}$ & $\begin{array}{c}N_{\mathrm{Gal}} \\
\left(10^{20} \mathrm{~cm}^{-2}\right) \\
(5)\end{array}$ & $\begin{array}{l}m_{V} \\
(6) \\
\end{array}$ & $\begin{array}{l}\text { Morph. } \\
\text { type } \\
(7) \\
\end{array}$ & $\begin{array}{c}\text { HBLR } \\
(8) \\
\end{array}$ & $\begin{array}{l}\text { Ref } \\
\text { (9) }\end{array}$ \\
\hline MARK 348 & 04847.2 & 315725 & 63.90 & 5.79 & 14.59 & S0-a & $\checkmark$ & 1 \\
\hline NGC 424 & 11127.7 & -3851 & 47.60 & 1.52 & 14.12 & SO-a & $\checkmark$ & 1 \\
\hline MARK 573 & 14357.8 & 22059 & 71.30 & 2.52 & 14.07 & SO-a & $\checkmark$ & 1 \\
\hline NGC 788 & 216.5 & -64856 & 56.10 & 2.11 & 12.76 & S0-a & $\checkmark$ & 1 \\
\hline ESO 417-G06 & 25621.5 & -32116 & 65.60 & 2.06 & 14.30 & SO-a & - & \\
\hline MARK 1066 & 25958.6 & 364914 & 51.70 & 9.77 & 13.96 & S0-a & $x$ & 2 \\
\hline $3 C 98.0$ & 35854.5 & 10262 & 124.90 & 10.20 & 15.41 & $\mathrm{E}$ & - & \\
\hline MARK 3 & 61536.3 & 71215 & 63.20 & 9.67 & 13.34 & So & $\checkmark$ & 1 \\
\hline MARK 1210 & 845.9 & 5650 & 53.60 & 3.45 & 13.70 & - & $\checkmark$ & 2 \\
\hline NGC 3079 & 10158.5 & 554050 & 19.10 & 0.89 & 12.18 & SBcd & $x$ & 2 \\
\hline IC 2560 & 101619.3 & -333359 & 34.80 & 6.40 & 13.31 & $\mathrm{SBb}$ & - & \\
\hline NGC 3393 & 104823.4 & -25944 & 48.70 & 6.03 & 13.95 & $\mathrm{SBa}$ & - & \\
\hline NGC 4507 & 123536.5 & -395433 & 46.00 & 5.88 & 13.54 & $\mathrm{Sab}$ & $\checkmark$ & 1 \\
\hline NGC 4698 & 124822.9 & 82914 & 23.40 & 1.79 & 12.27 & $\mathrm{Sab}$ & - & \\
\hline NGC 5194 & 132952.4 & 471141 & 7.85 & 1.81 & 13.47 & $\mathrm{Sbc}$ & $x$ & 2 \\
\hline MARK 268 & 134111.1 & 302241 & 161.50 & 1.37 & 14.66 & SO-a & - & \\
\hline MARK 273 & 134442.1 & 555313 & 156.70 & 0.89 & 14.91 & $\mathrm{Sab}$ & - & \\
\hline Circinus & 14139.8 & -652017 & 4.21 & 74.40 & 12.1 & $\mathrm{Sb}$ & $\checkmark$ & 1 \\
\hline NGC 5643 & 143240.7 & -441028 & 16.90 & 7.86 & 13.60 & $\mathrm{Sc}$ & $x$ & 2 \\
\hline MARK 477 & 144038.1 & 533015 & 156.70 & 1.05 & 15.03 & E? & $\checkmark$ & 2 \\
\hline IC $4518 A$ & 145741.2 & -43756 & 65.20 & 8.21 & 15. & $\mathrm{Sc}$ & - & \\
\hline ESO 138-G01 & 165120.5 & -591411 & 36.00 & 13.10 & 13.63 & E-S0 & - & \\
\hline NGC 6300 & 171659.2 & -62495 & 14.43 & 7.76 & 13.08 & $\mathrm{SBb}$ & - & \\
\hline NGC 7172 & 2221.9 & -31528 & 33.90 & 1.48 & 13.61 & $\mathrm{Sa}$ & $x$ & 2 \\
\hline NGC 7212 & 2272.0 & 10140 & 111.80 & 5.12 & 14.8 & $\mathrm{Sb}$ & $\checkmark$ & 1 \\
\hline NGC 7319 & 22363.5 & 335833 & 77.25 & 6.15 & 13.53 & $\mathrm{Sbc}$ & - & \\
\hline
\end{tabular}

Notes. ${ }^{(1)}$ All distances are taken from the NED and correspond to the average redshift-independent distance estimates. Column 1: Name; Col. 2: right ascension; Col. 3: declination; Col. 4: distance; Col. 5: galactic absorption; Col. 6: aparent magnitude in the Johnson filter $V$ from Véron-Cetty \& Véron (2010); Col. 7: galaxy morphological type from Hyperleda; Col. 8: hidden broad polarized lines detected.

References. (1) Véron-Cetty \& Véron (2010); (2) Gu \& Huang (2002).

\subsection{XMM-Newton data}

$X M M-N e w t o n$ observations were obtained with the EPIC pn camera (Strüder et al. 2001). The data were reduced in a systematic, uniform way using the Science Analysis Software (SAS ${ }^{6}$ ), version 11.0.0. First, good-timing periods were selected using a method that maximizes the signal-to-noise ratio of the net source spectrum by applying a different constant count rate threshold on the single events, $E>10 \mathrm{keV}$ field-of-view background light curve. We extracted the spectra of the nuclei from circles of $15-30^{\prime \prime}$ (or 300-600 px) radius centered on the positions given by NED, while the backgrounds were extracted from circular regions using an algorithm that automatically selects the best area - and closest to the source - that is free of sources. This selection was manually checked to ensure the best selection for the backgrounds.

The source and background regions were extracted with the EVSELECT task. The response matrix files (RMF) and the ancillary response files (ARF) were generated using the RMFGEN and ARFGEN tasks, respectively. To be able to use the $\chi^{2}$ statistics, the spectra were binned to obtain at least 20 counts per spectral bin using the GRPPHA task.

\subsection{Light curves}

Light curves in three energy bands $(0.5-2.0 \mathrm{keV}, 2.0-10.0 \mathrm{keV}$, and $0.5-10 \mathrm{keV}$ ) for the source and background regions as

\footnotetext{
6 http://xmm.esa.int/sas/
}

defined above were extracted using the DMEXTRACT task (for XMM-Newton) and EVSELECT task (for Chandra) with a $1000 \mathrm{~s}$ bin. To be able to compare the variability amplitudes in different light curves of the same object, only those observations with a net exposure time longer than $30 \mathrm{ks}$ were taken into account. For longer observations, the light curves were divided into segments of $40 \mathrm{ks}$, so in some cases more than one segment of the same light curve can be extracted. Intervals with flare-like events and/or prominent decreasing/increasing trends were manually rejected from the source light curves. We notice that after excluding these events, the exposure time of the light curve could be shorter, thus we recall that only observations with a net exposure time longer than $30 \mathrm{ks}$ were used for the analysis. The light curves are shown in Appendix D. We recall that these values are used only for visual inspection of the data and not as estimators of the variability (as in Hernández-García et al. 2014).

\section{Methodology}

The methodology is explained in Hernández-García et al. (2013) and Hernández-García et al. (2014). In contrast to the study of LINER nuclei, we added a new model (namely 2ME2PL), and a cold reflection component for the individual spectral fittings and an analysis of the Compton-thickness for the Seyfert galaxies. Additionally, we changed the way we estimate the nuclear contribution in XMM-Newton spectra to perform the simultaneous fit using different instruments (see Sect. 4.2). A comparison 
with a sample of LINERs will be performed in a forthcoming paper. For clarity, we recall the procedure below.

\subsection{Individual spectral analysis}

An individual spectral analysis allowed us to select the bestfit model for each data set. We added a new model with respect to previous works (2ME2PL), including an additional thermal component to the more complex model, ME2PL, to explain the two ionized zones observed in some Seyfert galaxies (e.g., Netzer \& Turner 1997; Bianchi et al. 2010). Then, we also added a cold reflection component (PEXRAV in XSPEC, Magdziarz \& Zdziarski 1995) to the best-fit model to check whether this component improves the fit. We used $\mathrm{XSPEC}^{7}$ version 12.7.0 to fit the data with six different models:

- PL: a single power law representing the continuum of a nonstellar source. The empirical model is $\mathrm{e}^{N_{\mathrm{Gal}} \sigma(E)} \cdot \mathrm{e}^{N_{\mathrm{H}} \sigma(E(1+z))}\left[N_{\mathrm{H}}\right] \cdot$ Norme $^{-\Gamma}[\Gamma$, Norm $]$.

- ME: the emission is dominated by hot diffuse gas, i.e., a thermal plasma. A MEKAL (in XSPEC) model is used to fit the spectrum. The model is $\mathrm{e}^{N_{\mathrm{Gal}} \sigma(E)} \cdot \mathrm{e}^{N_{\mathrm{H}} \sigma(E(1+z))}\left[N_{\mathrm{H}}\right] \cdot M E K A L[k T, N o r m]$.

- 2PL: in this model the primary continuum is an absorbed power law representing the non stellar source, while the soft energies are due to a scattering component that is represented by another power law. Mathematically the model is explained as

$\mathrm{e}^{N_{\mathrm{Gal}} \sigma(E)}\left(\mathrm{e}^{N_{\mathrm{H} 1} \sigma(E(1+z))}\left[N_{\mathrm{H} 1}\right] \cdot\right.$ Norm $_{1} \mathrm{e}^{-\Gamma}\left[\Gamma\right.$, Norm $\left._{1}\right]+$ $\mathrm{e}^{N_{\mathrm{H} 2} \sigma(E(1+z))}\left[N_{\mathrm{H} 2}\right] \cdot \operatorname{Norm}_{2} \mathrm{e}^{-\Gamma}\left[\Gamma\right.$, Norm $\left.\left._{2}\right]\right)$.

- MEPL: the primary continuum is represented by an absorbed power law, but at soft energies a thermal plasma dominates the spectrum. Empirically it can be described as

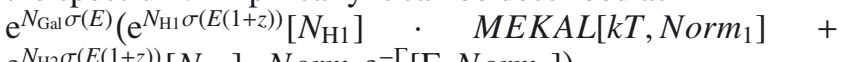
$\mathrm{e}^{N_{\mathrm{H} 2} \sigma(E(1+z))}\left[N_{\mathrm{H} 2}\right] \cdot$ Norm $_{2} \mathrm{e}^{-\Gamma}\left[\Gamma\right.$, Norm $\left.\left._{2}\right]\right)$.

- ME2PL: this is the same model as MEPL, but an additional power law is required to explain the scattered component at soft energies, so mathematically it is $\mathrm{e}^{N_{\mathrm{Gal}} \sigma(E)}\left(\mathrm{e}^{N_{\mathrm{H} 1} \sigma(E(1+z))}\left[N_{\mathrm{H} 1}\right] \cdot \operatorname{Norm}_{1} \mathrm{e}^{-\Gamma}\left[\Gamma\right.\right.$, Norm $\left._{1}\right]+$ MEKAL $[k T]+\mathrm{e}^{N_{\mathrm{H} 2} \sigma(E(1+z))}\left[N_{\mathrm{H} 2}\right] \cdot$ Norm $_{2} \mathrm{e}^{-\Gamma}\left[\Gamma\right.$, Norm $\left.\left._{2}\right]\right)$.

- 2ME2PL: the hard X-ray energies are represented by an absorbed power law, while the spectrum shows a complex structure at soft energies, where a composite of two thermal plasmas plus a power law are required. In Seyfert galaxies, at least two ionized phases (a warm and a hot) are required to properly fit their spectra (Netzer \& Turner 1997), which is confirmed by high resolution data (e.g., Bianchi et al. 2010; Marinucci et al. 2011). Ideally, the spectral fit should be made by using photoionization models to fit high quality data (e.g., RGS) and then use the obtained spectral parameters to fit lower quality data, as in Bianchi et al. (2010) or González-Martín et al. (2010). We tried to use photoionized models using Cloudy to fit the soft emission. We found that, due to the low resolution of our data, these models fit the data similarly to MEKAL models. Therefore, for simplicity, in this work we represent the photoionized gas by two thermal plasmas plus Gaussian lines when required (see below). The power law at soft energies represents the scattering component. Although this is probably a simple model for fitting the complexity of the spectra, the data analyzed in this

7 http://heasarc.nasa.gov/xanadu/xspec/ work do not have enough spectral resolution to properly fit the data with more realistic models, and therefore this model is enough for our purposes. It is represented as $\mathrm{e}^{N_{\mathrm{Gal}} \sigma(E)}\left(\mathrm{e}^{N_{\mathrm{H} 1} \sigma(E(1+z))}\left[N_{\mathrm{H} 1}\right]\right.$. Norm $_{1} \mathrm{e}^{-\Gamma}\left[\Gamma\right.$, Norm $\left._{1}\right]+$ $M E K A L\left[k T_{1}\right]+M E K A L\left[k T_{2}\right]+\mathrm{e}^{N_{\mathrm{H} 2} \sigma(E(1+z))}\left[N_{\mathrm{H} 2}\right]$. Norm $_{2} \mathrm{e}^{-\Gamma}\left[\Gamma\right.$, Norm $\left.\left._{2}\right]\right)$.

- (Best-fit model) + PEXRAV: from the six models described above, we selected the one that provided the best fit to the data and added a reflection component (we have chosen PEXRAV within XSPEC) to account for a plausible contribution of this component in highly obscured Seyfert 2s. The parameters of the MEKAL component(s) were frozen to the best-fit values. In this model the absorbed power law at hard energies represents the transmitted component, while the PEXRAV is indicative of the reflected fraction from the primary continuum alone, by setting the reflection scaling factor to 1 . The spectral index was set to be that of the power law(s), the exponential cutoff was fixed to $200 \mathrm{keV}$, and the inclination angle to $45^{\circ}$. These parameters are based on typical values obtained from X-ray analyses at harder energies (e.g., Guainazzi et al. 2005b; Matt et al. 2004; Akylas \& Georgantopoulos 2009; Noguchi et al. 2009). The free parameters in this model are therefore

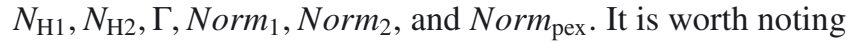
that we tried similar models to fit the data, such as exchanging the hard PL by PEXRAV or by an absorbed PEXRAV, and obtained very similar results, but the model explained above allowed the use of the F test to check for eventual improvements in the fits.

In the equations above, $\sigma(E)$ is the photo-electric cross-section, $z$ is the redshift, and $\operatorname{Norm}_{i}$ are the normalizations of the power law, the thermal component, or the reflected component (i.e., $\mathrm{Norm}_{1}, \mathrm{Norm}_{2}$, and Norm ters that vary are written in brackets. The Galactic absoption, $N_{\text {Gal }}$, is included in each model and fixed to the predicted value (Col. 5 in Table 1) using the tool NH within FTOOLS (Dickey \& Lockman 1990; Kalberla et al. 2005). Even if not included in the mathematical form above, all the models include three narrow Gaussian lines to take the iron lines at $6.4 \mathrm{keV}(\mathrm{FeK} \alpha)$, $6.7 \mathrm{keV}$ (FeXXV), and $6.95 \mathrm{keV}$ (FeXXVI) into account. In a few cases, additional Gaussian lines were required at soft energies from a visual inspection, including $\mathrm{Ne} \mathrm{X}$ at $1.2 \mathrm{keV}, \mathrm{Mg} \mathrm{XI}$ at $1.36 \mathrm{keV}$, Si XIII at $1.85 \mathrm{keV}$, and S XIV at $2.4 \mathrm{keV}$.

The $\chi^{2} /$ d.o.f. and $\mathrm{F}$ test were used to select the simplest model that represents the data best.

\subsection{Simultaneous spectral analysis}

Once the individual best-fit model is selected for each observation, and if the models are different for the individual observations, then the most complex model that fits each object was chosen. This model was used to simultaneously fit spectra obtained at different dates of the same nuclei. Initially, the values of the spectral parameters were set to those obtained for the spectrum with the largest number counts for each galaxy. To determine whether spectral variations are observed in the data, this simultaneous fit was made in three steps:

0. SMF0 (Simultaneous fit 0): the same model was used with all parameters linked to the same value to fit every spectra of the same object, i.e., the non-variable case.

1. SMF1: Using SMF0 as the baseline for this step, we let the parameters $N_{\mathrm{H} 1}, N_{\mathrm{H} 2}, \Gamma$, Norm $_{1}$, Norm $_{2}$, Norm $_{\text {pex }}, k T_{1}$, and 
$k T_{2}$ vary individually. The best fit was selected for the $\chi_{\mathrm{r}}^{2}$ closest to unity that improved SMF0 (using the F test).

2. SMF2: Using SMF1 as the baseline for this step (when SMF1 did not fit the data well), we let two parameters vary, the one that varied in SMF1 along with any of the other parameters of the fit. The $\chi_{\mathrm{r}}^{2}$ and $\mathrm{F}$ test were again used to confirm an improvement in the fit.

When data from the same instrument were available at different epochs, this method was applied separately for Chandra and/or XMM-Newton. However, in some cases only one observation was available per instrument. Instead of directly comparing the spectra from different instruments, we tried to decontaminate the extranuclear emission in XMM-Newton data, to make sure that the emission included in the larger aperture did not produce the observed variability. This additional analysis was performed by extracting an annular region from Chandra data, fitting the models explained above to its spectrum, and selecting the one that best fits the annular region. This model was later incorporated into the XMM-Newton spectrum (with its parameters frozen), so the parameters of the nuclear emission can be estimated. We determined the contribution by the annular region to the Chandra data from the number counts (i.e., modelindependent) in the $0.5-10.0 \mathrm{keV}$ energy band, and this percentage was used to estimate the number counts in the nuclear region of XMM-Newton data. Following the same criteria as we used to select the data (see Sect. 2), data from different instruments were compared when the number counts in the nuclear XMM-Newton spectrum was more than 400 counts. We note that this procedure differs from the one used in Hernández-García et al. (2013, 2014). When multiple observations of the same object and instrument were available, we compared the data with the closest dates (marked with $c$ in Table A.1).

\subsection{Flux variability}

The luminosities in the soft and hard X-ray energy bands were computed using XSPEC for both the individual and the simultaneous fits. For their calculation, we took the distances from NED, corresponding to the average redshift-independent distance estimate for each object, when available, or to the redshiftestimated distance otherwise; distances are listed in Table 1.

When data from the optical monitor (OM) onboard $X M M-N e w t o n$ were available, UV luminosities (simultaneously to X-ray data) were estimated in the available filters. We recall that UVW2 is centered at $1894 \AA(1805-2454) \AA$, UVM2 at $2205 \AA(1970-2675) \AA$, and UVW1 at $2675 \AA(2410-3565) \AA$. We used the OM observation FITS source lists (OBSMLI) ${ }^{8}$ to obtain the photometry. When OM data were not available, we searched for UV information in the literature. We note that in this case, the X-ray and UV data might not be simultaneous (see Appendix B).

We assumed an object to be variable when the square root of the squared errors was at least three times smaller than the difference between the luminosities (see Hernández-García et al. 2014 , for details).

\subsection{Short-term variability}

Firstly, we assumed a constant count rate for segments of 30-40 ks of the observation in each energy band and calculated

\footnotetext{
8 ftp://xmm2.esac. esa.int/pub/odf/data/docs/ XMM-SOC-GEN-ICD-0024.pdf
}

$\chi^{2} /$ d.o.f. as a proxy to the variations. We considered the source as a candidate for variability if the count rate differed from the average by more than $3 \sigma$ (or $99.7 \%$ probability).

Secondly, and to be able to compare the variability amplitude of the light curves between observations, we calculated the normalized excess variance, $\sigma_{\mathrm{NXS}}^{2}$, for each light curve segment with 30-40 ks following prescriptions in Vaughan et al. (2003; see also González-Martín et al. 2011b; Hernández-García et al. 2014). We recall that $\sigma_{\text {NXS }}^{2}$ is related to the area below the power spectral density (PSD) shape.

When $\sigma_{\text {NXS }}^{2}$ was negative or compatible with zero within the errors, we estimated the $90 \%$ upper limits using Table 1 in Vaughan et al. (2003). We assumed a PSD slope of -1, the upper limit from Vaughan et al. (2003), and we added the value of $1.282 \mathrm{err}\left(\sigma_{\mathrm{NXS}}^{2}\right)$ to the limit to account for Poisson noise. For a number of segments, $N$, obtained from an individual light curve, an upper limit for the normalized excess variance was calculated. When $N$ segments were obtained for the same light curve and at least one was consistent with being variable, we calculated the normalized weighted mean and its error as the weighted variance.

We considered short-term variations for $\sigma_{\mathrm{NXS}}^{2}$ detections above $3 \sigma$ of the confidence level.

\subsection{Compton thickness}

Highly obscured AGN are observed through the dusty torus, in some cases with column densities higher than $1.5 \times 10^{24} \mathrm{~cm}^{-2}$ (the so-called Compton-thick). In these cases the primary emission can be reflected at energies $\sim 10 \mathrm{keV}$. Since the primary continuum cannot be directly observed, some indicators using X-rays and [O III] data have been used to select candidates (Ghisellini et al. 1994; Bassani et al. 1999; Panessa \& Bassani 2002; Cappi et al. 2006).

To properly account for the slope of the power law, $\Gamma$, and the equivalent width of the iron line, $\mathrm{EW}(\mathrm{FeK} \alpha)$, an additional analysis was performed. We fit the 3-10 keV energy band of each spectrum individually with a PL model (see Sect. 4.1) to obtain the values of $\Gamma$ and $\mathrm{EW}(\mathrm{FeK} \alpha)$. Compton-thick candidates can be selected by using three different criteria:

$-\Gamma<1$ : since the transmitted component is suppressed below $10 \mathrm{keV}$, a flattening of the observed spectrum is expected (Cappi et al. 2006; González-Martín et al. 2009a).

- $E W(\mathrm{FeK} \alpha)>500 \mathrm{eV}$ : if the nuclear emission is obscured by a Compton-thick column density, the primary continuum underneath the $\mathrm{FeK} \alpha$ line is strongly suppressed, and the equivalent width of the line enhanced to $\sim \mathrm{keV}$ (Krolik et al. 1994; Ghisellini et al. 1994).

- $F(2-10 \mathrm{keV}) / F_{[\mathrm{OIII}]}<1$ : since the primary continuum is suppressed, the X-ray luminosity is underestimated, so when comparing with an isotropic indicator of the AGN power (as is the case for the [O III] emission line), the ratio between the two values decreases (Bassani et al. 1999; Guainazzi et al. 2005b; Cappi et al. 2006; González-Martín et al. 2009a). Thus, we have used this ratio to select Compton-thick candidates, where the extinction-corrected [O III] fluxes were obtained from the literature (and corrected when needed following Bassani et al. 1999), and the hard X-ray luminosities, $L(2-10 \mathrm{keV}$ ), from the individual fits were used (see Table A.3) for the calculation.

We considered that a source is a Compton-thick candidate when at least two of the three criteria above were met. Otherwise, 

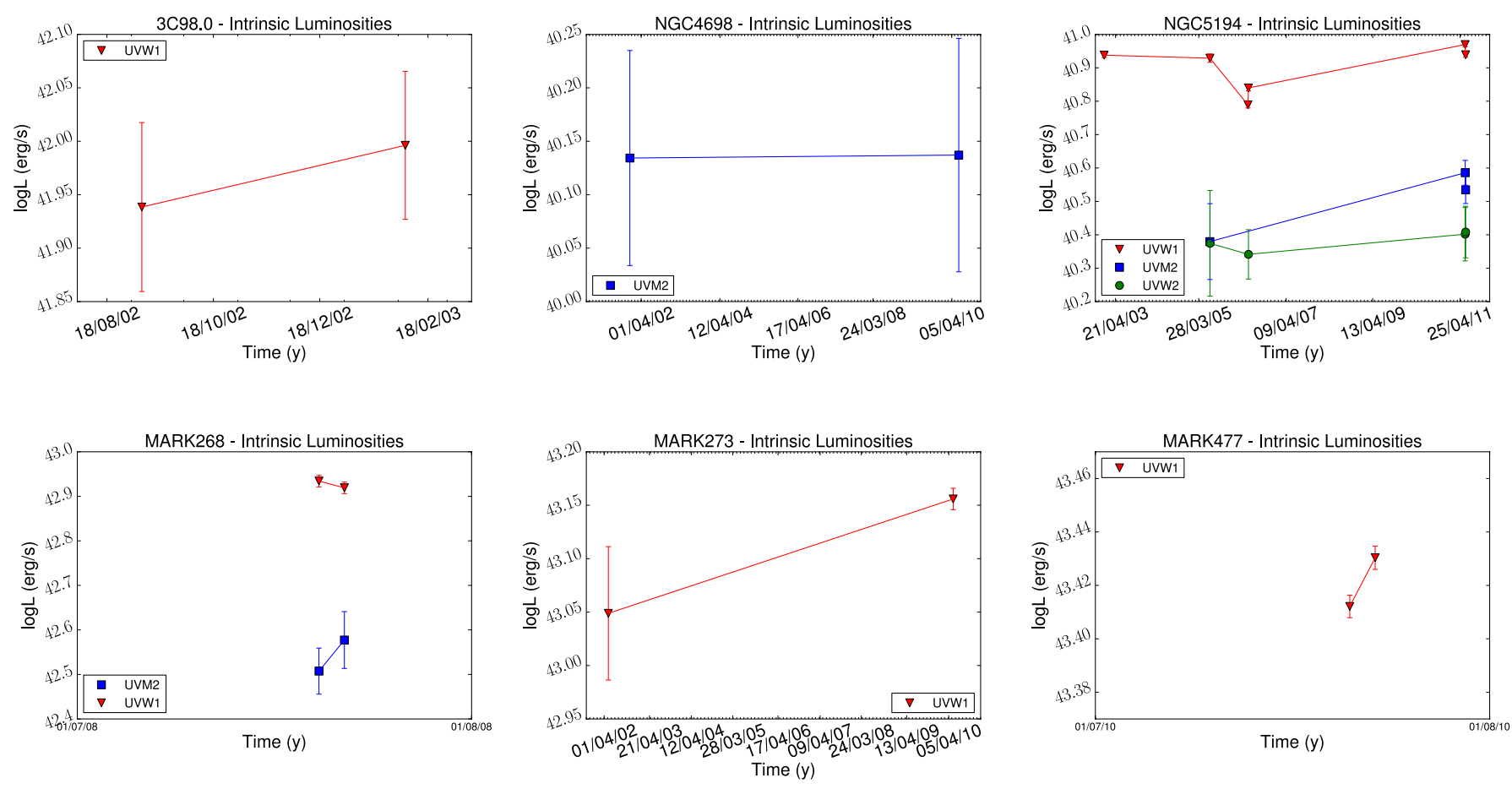

Fig. 1. UV luminosities obtained from the data with the OM camera onboard XMM-Newton, when available. Different filters have been used; UVW1 (red triangles), UVW2 (green circles), and UVM2 (blue squares).

the source is considered to be a Compton-thin candidate. When different observations of the same source result in different classifications, the object was considered to be a changing-look candidate.

The spectral fits reported in Sects. 4.1 and 4.2 are performed with the spectral indices of the soft, $\Gamma_{\text {soft }}$, and the hard, $\Gamma_{\text {hard }}$, power laws tied to the same value. When a source is Comptonthick, its spectrum is characterized by a flat power law at hard energies (see above), whereas the slope of the power law is dominated by the scattered component if we tied $\Gamma_{\text {soft }}=\Gamma_{\text {hard }}$, giving an unrealistic steep power-law index. Thus, the simultaneous analysis was repeated by leaving $\Gamma_{\text {soft }}$ and $\Gamma_{\text {hard }}$ free for the objects classified as Compton-thick candidates. We first made the SMF1 with $\Gamma_{\text {hard }}$ vary and found that this component does not vary in any case. The values of $\Gamma_{\text {hard }}$ obtained for the Compton-thick candidates following this procedure are reported in Table A.7 (Col. 9). We checked that the rest of the parameters in the model are consistent with those reported in Table A. 2 within the uncertainties. The same procedure was applied to Compton-thin candidates, and compatible values of $\Gamma_{\text {soft }}$ and $\Gamma_{\text {hard }}$ were found. It is worth pointing out that it is not within the scope of this work to obtain the best spectral parameters for each source, but to obtain their variability patterns. Thus, we have kept the same general analysis for all the objects (i.e., with $\Gamma_{\text {soft }}=\Gamma_{\text {hard }}$, although we notice that this is not the case for Compton-thick candidates), but this procedure does not affect the main results presented in this paper.

\section{Results}

In this section we present the results for the variability analysis of the Seyfert 2 galaxies individually (see Sect. 5.1), as well as the general results, including the characterization of the spectra of Seyfert 2s (Sect. 5.2), the long-term variability (Sect. 5.3), first for the whole sample in general and later divided into subsamples, X-ray short-term variations (Sect. 5.4), and flux variations at UV frequencies (Sect. 5.5). The main results of the analysis are summarized in Table 2. Individual notes on each galaxy and comparisons with previous works can be found in Appendix B.

\subsection{Individual objects}

For details on the data and results, we refer the reader to the following tables and figures: the observations used in the analysis (Table A.1); UV luminosities with simultaneous OM data (Col. 9 of Table A.1 and Fig. 1); individual and simultaneous best fit, and the parameters varying in the model (Table A.2 and Fig. 2); X-ray flux variations (Table A.3 and Fig. 3); comparison of Chandra and XMM-Newton data using the annular region (Table A.4); the simultaneous fit between these observations (Table A.5 and Figs. A.1 and A.2); short-term variability from the analysis of the light curves (Table A.6 and Appendix D); and the Compton-thickness analysis, where an object was classified on the basis that at least two of the three criteria presented in Sect 4.5 were met (Table A.7). We notice that the addition of a cold reflection component is not statistically required by the data, so we do not mention the analysis except in one case (3C 98.0) where the simultaneous fit was performed.

- MARK 348: SMF1 with variations in Norm $_{2}$ (69\%) represents the data best. These variations were found within a nine-year period, which implies intrinsic flux variations of $69 \%(68 \%)$ in the soft (hard) energy band. We classify it as a Compton-thin candidate.

- NGC 424: two XMM-Newton data sets are available. SMF0 results in $\chi_{\mathrm{r}}^{2}=2.20$, and SMF1 does not improve the fit; this is most probably because the spectra from 2008 shows a more complex structure compared to 2000, preventing a proper simultaneous spectral fitting. Thus, we do not perform the simultaneous spectral fit between the two $X M M-N e w t o n$ data sets. The contribution from the annular 
L. Hernández-García et al.: X-ray variability of Seyfert 2s

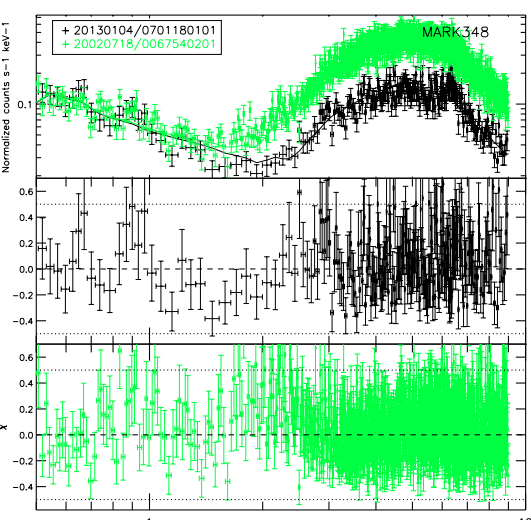

Energy $(k \in \mathrm{V})$

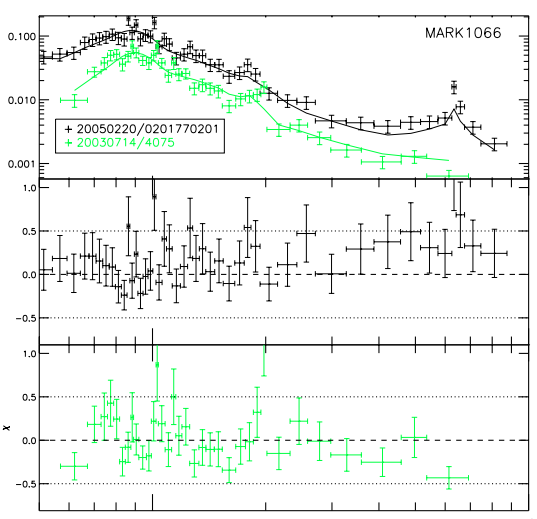

Energy (keV)
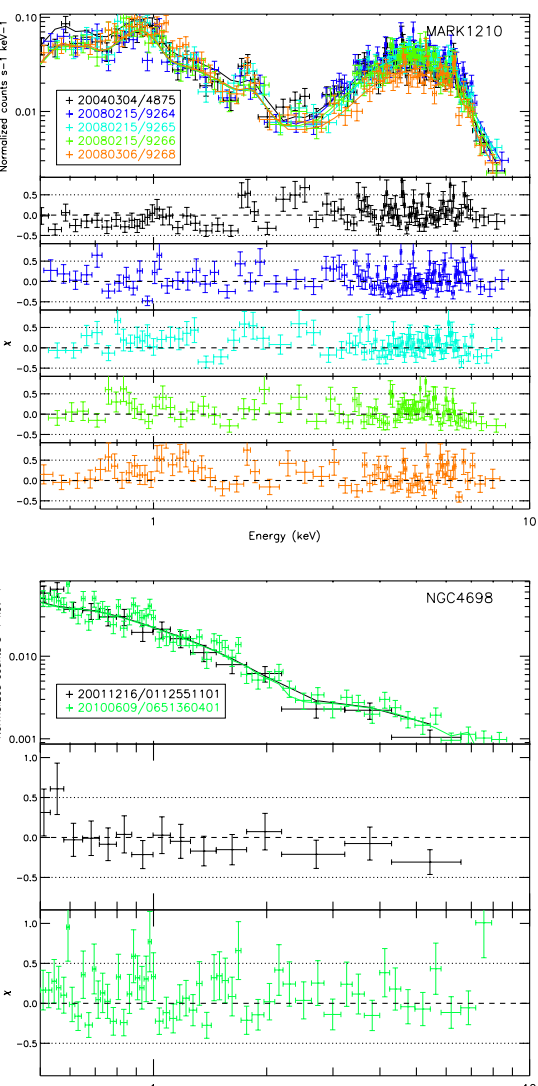

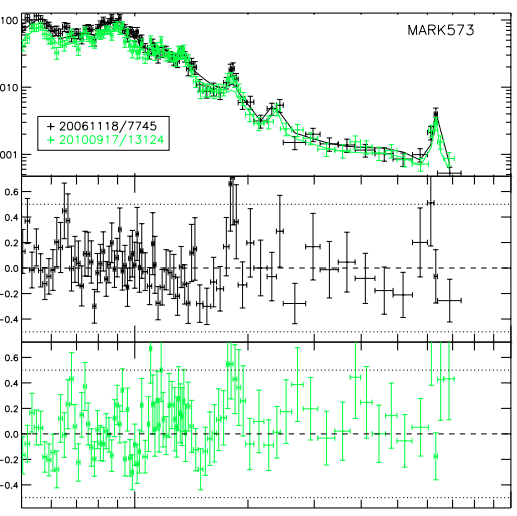

Energy (keV)

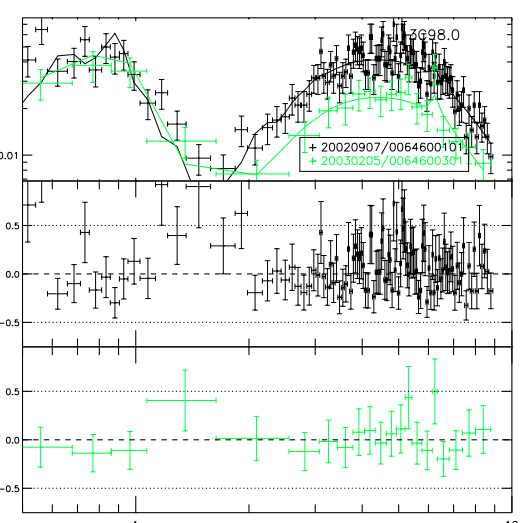

Energy (kev)

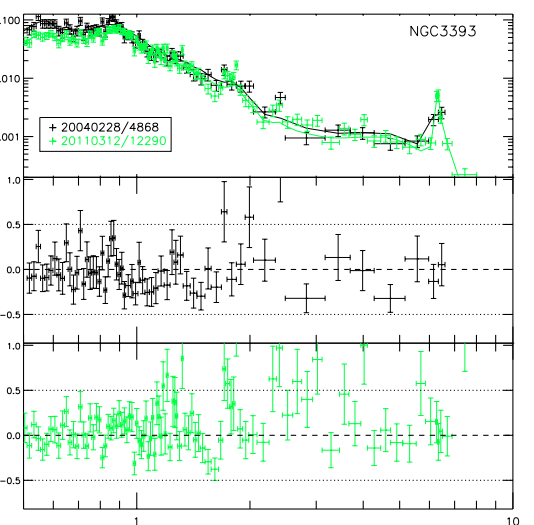

Energy (kev)

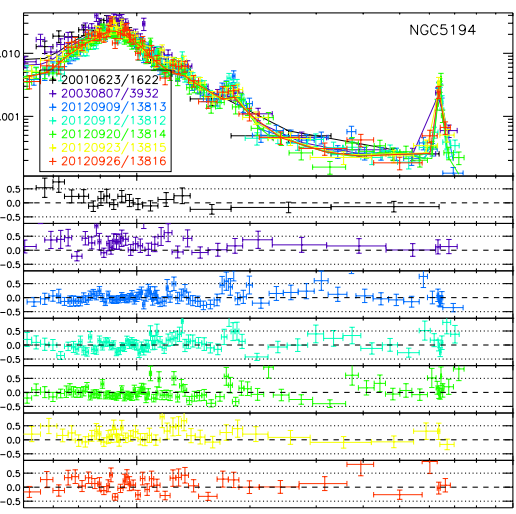

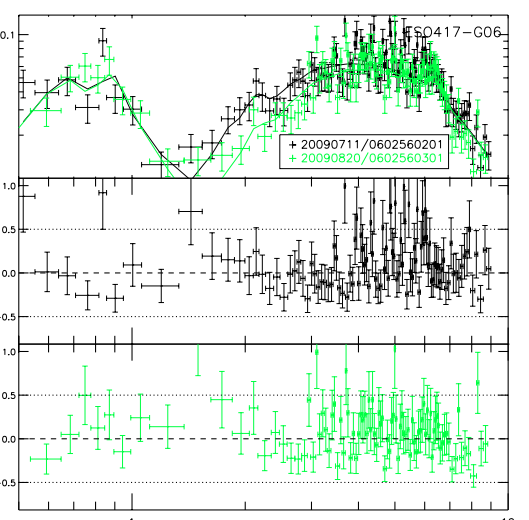

Energy (keV)

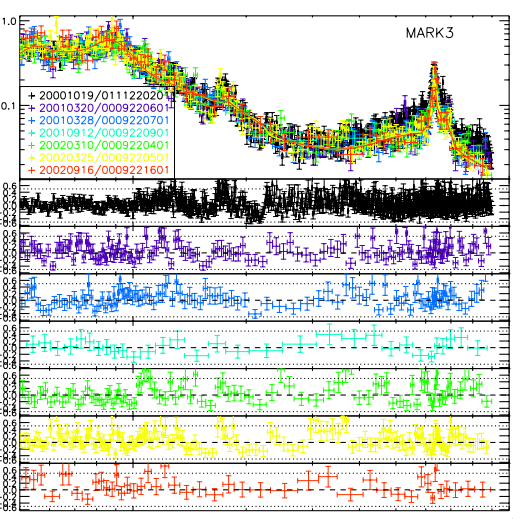

Energy (keV)
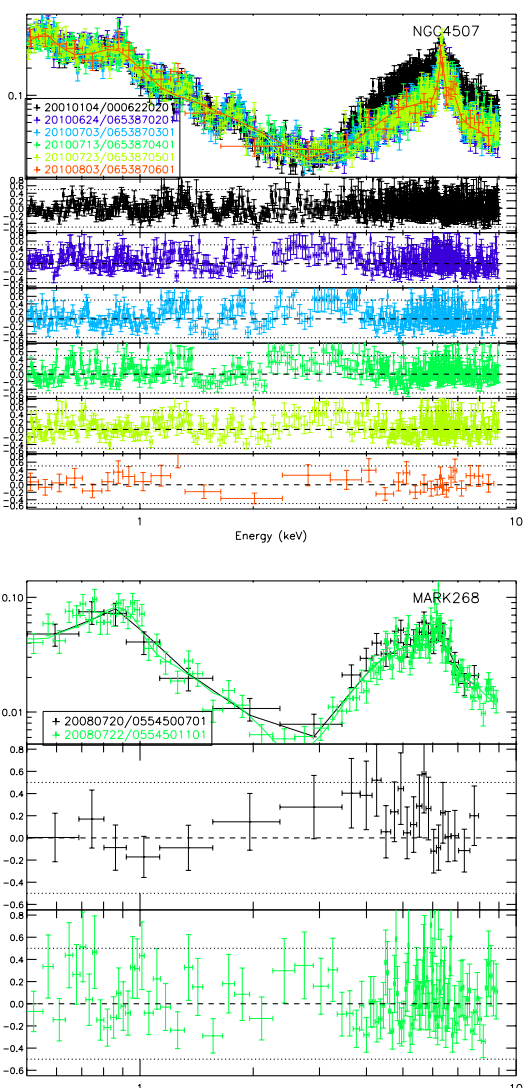

Fig. 2. For each object, top: simultaneous fit of X-ray spectra; from second row on: residuals in units of $\sigma$. The legends contain the date (in the format yyyymmdd) and the obsID. Details are given in Table 1 . 

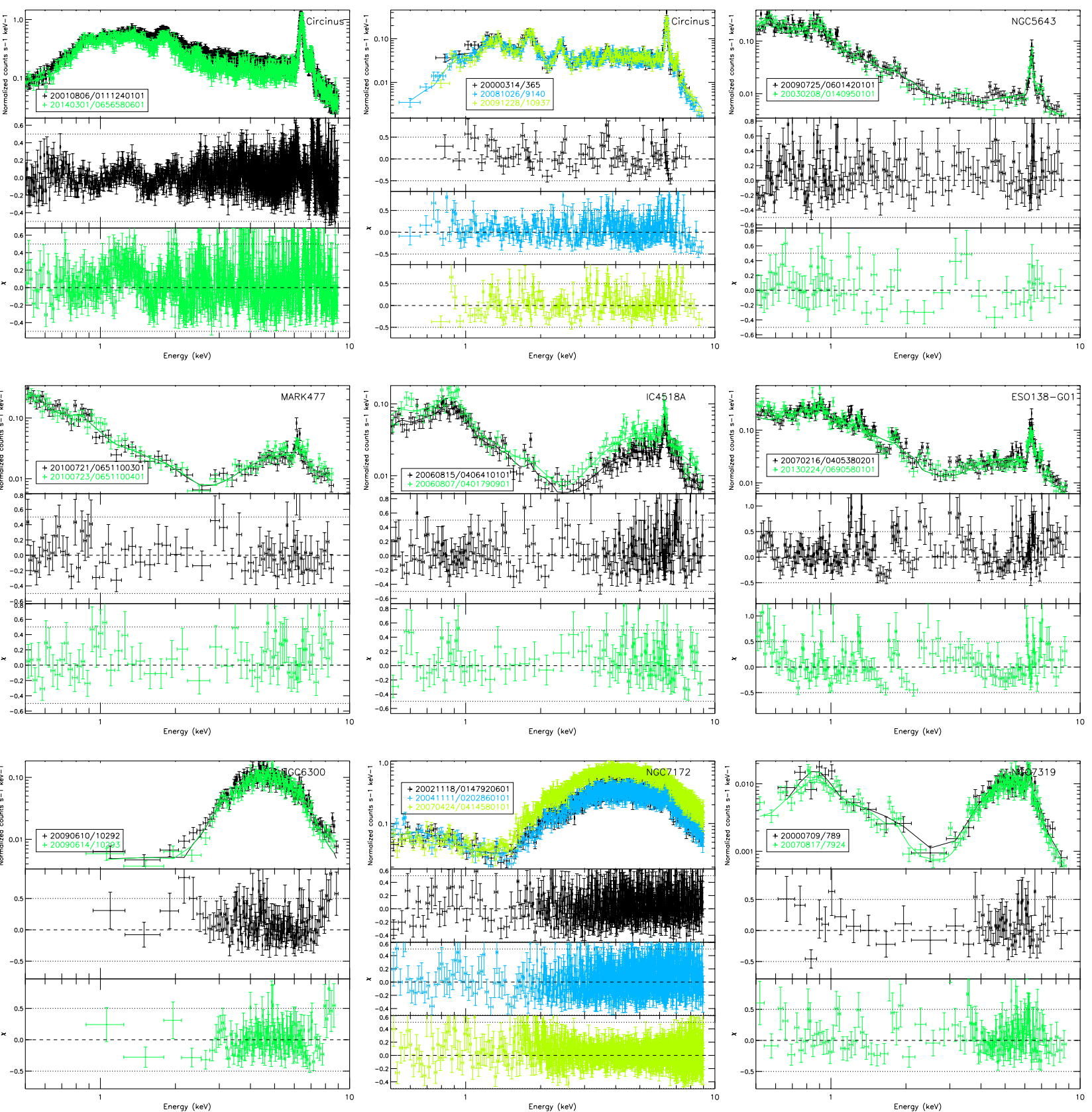

Fig. 2. continued.

region is negligible, thus the spectral analysis can be jointly performed using XMM-Newton and Chandra data together. SMF0 is the best representation of the data. Short-term variations from the XMM-Newton light curve are not found. We classify it as a Compton-thick candidate.

- MARK 573: the Chandra data do not show variations (SMF0 was used) within a four-year period. When compared with XMM-Newton data, the annular region contributes with $24 \%$ to the Chandra data. Again, SMF0 results in the best representation of the data. Three additional Gaussian lines are needed to fit the data at $1.20 \mathrm{keV}(\mathrm{Ne} \mathrm{X}), 1.36 \mathrm{keV}(\mathrm{Mg} \mathrm{XI})$, and $2.4 \mathrm{keV}$ (S XIV). Two Chandra light curves are analyzed, and variations are not detected. We classify it as a Compton-thick candidate.

- NGC 788: one observation per instrument is available. The emission from the annular region is negligible so we jointly fit Chandra and XMM-Newton data. SMF0 was used, thus no variations are found in a two years period. We classify it as a Compton-thin candidate.

- ESO 417-G06: SMF1 with $N_{\mathrm{H} 2}(21 \%)$ because the parameter varying represents the data best. These variations were obtained within about a one-month period, corresponding to no flux intrinsic variations. We classify it as a Compton-thin candidate.

- MARK 1066: only one observation per instrument is available. The annular region contributes with $8 \%$ to Chandra data. The simultaneous fit without allowing any parameter to vary (i.e., SMF0) results in a good fit of the data. We classify it as a Compton-thick candidate.

- 3C98.0: this is the only object where the unabsorbed PEXRAV component improves the fit. The values of the spectral parameters in this fit are 
Table 2. Results of the variability analysis.

\begin{tabular}{|c|c|c|c|c|c|c|c|c|c|}
\hline \multirow{2}{*}{$\begin{array}{l}\text { Name } \\
\text { (1) }\end{array}$} & \multirow{2}{*}{$\begin{array}{l}\text { Type } \\
\text { (2) }\end{array}$} & \multirow{2}{*}{$\begin{array}{c}\log \left(L_{\mathrm{soft}}\right) \\
(0.5-2 \mathrm{keV}) \\
(3)\end{array}$} & \multirow{2}{*}{$\begin{array}{c}\log \left(L_{\text {hard }}\right) \\
(2-10 \mathrm{keV}) \\
(4)\end{array}$} & \multirow{2}{*}{$\begin{array}{c}\log \left(M_{\mathrm{BH}}\right) \\
(5)\end{array}$} & \multirow{2}{*}{$\begin{array}{c}\log \left(R_{\mathrm{Edd}}\right) \\
(6)\end{array}$} & \multicolumn{3}{|c|}{ Variability } & \multirow{2}{*}{$\begin{array}{c}\Delta T_{\max } \\
\text { (Years) } \\
\text { (10) }\end{array}$} \\
\hline & & & & & & $\begin{array}{l}\text { SMF0 } \\
(7)\end{array}$ & $\begin{array}{c}\text { SMF1 } \\
(8)\end{array}$ & $\begin{array}{c}\text { SMF2 } \\
(9)\end{array}$ & \\
\hline MARK $348(\mathrm{X})$ & HBLR & $\begin{array}{c}42.76 \\
69_{-}^{+} 5 \%\end{array}$ & $\begin{array}{c}43.15 \\
68_{-}^{+} 3 \%\end{array}$ & 7.58 & -1.02 & ME2PL & $\begin{array}{l}\mathrm{Norm}_{2} \\
69^{+17} \%\end{array}$ & - & 10 \\
\hline NGC $424(\mathrm{C}, \mathrm{X})^{*}$ & HBLR & $\begin{array}{c}41.74 \\
0 \%\end{array}$ & $\begin{array}{c}41.85 \\
0 \%\end{array}$ & 7.78 & -2.53 & 2ME2PL & -14 & - & 0.16 \\
\hline MARK 573 (C)* & HBLR & $\begin{array}{c}41.65 \\
0 \%\end{array}$ & $\begin{array}{l}41.54 \\
0 \%\end{array}$ & 7.37 & -2.42 & $\begin{array}{l}\text { 2ME2PL } \\
\text { (+3gauss) }\end{array}$ & - & - & 4 \\
\hline$(\mathrm{X}, \mathrm{C})$ & & $\begin{array}{c}41.73 \\
0 \%\end{array}$ & $\begin{array}{l}41.41 \\
0 \%\end{array}$ & & & 2ME2PL & - & - & 2 \\
\hline NGC $788(X, C)$ & HBLR & $\begin{array}{c}42.11 \\
0 \%\end{array}$ & $\begin{array}{c}42.60 \\
0 \%\end{array}$ & 7.43 & -1.43 & 2ME2PL & - & - & 0.33 \\
\hline ESO 417-G06 (X) & - & $\begin{array}{c}42.46 \\
0 \%\end{array}$ & $\begin{array}{l}42.50 \\
0 \%\end{array}$ & 7.44 & -1.53 & MEPL & $\begin{array}{c}N_{\mathrm{H} 2} \\
21_{-5}^{+5} \%\end{array}$ & - & 0.08 \\
\hline MARK $1066(\mathrm{X}, \mathrm{C})^{*}$ & NHBLR & $\begin{array}{c}41.40 \\
0 \%\end{array}$ & $\begin{array}{c}41.43 \\
0 \%\end{array}$ & 7.23 & -2.38 & ME2PL & - & - & 2 \\
\hline 3C $98.0(\mathrm{X})$ & - & $\begin{array}{l}43.13 \\
5_{-4}^{+} \%\end{array}$ & $\begin{array}{c}42.80 \\
42_{-}^{+} 7 \%\end{array}$ & 7.75 & -1.73 & MEPL & $\begin{array}{l}\mathrm{Norm}_{2} \\
43^{+41 \%} \%\end{array}$ & - & 0.41 \\
\hline$(\mathrm{X}, \mathrm{C})$ & & $\begin{array}{c}42.40 \\
0 \%\end{array}$ & $\begin{array}{c}42.60 \\
0 \%\end{array}$ & & & MEPL & -26 & - & 5 \\
\hline MARK $3(\mathrm{X}) *$ & HBLR & $\begin{array}{c}42.24 \\
29_{-}^{+} 7 \%\end{array}$ & $\begin{array}{c}42.74 \\
32_{-}^{+} 4 \%\end{array}$ & 8.74 & -2.58 & 2ME2PL & $\begin{array}{l}\mathrm{Norm}_{2} \\
37_{-14}^{+16 \%}\end{array}$ & - & 1 \\
\hline MARK 1210 (C) & HBLR & $\begin{array}{l}42.31 \\
7+5 \%\end{array}$ & $\begin{array}{l}42.79 \\
7_{-1}^{+} 1 \%\end{array}$ & 7.70 & -1.50 & 2ME2PL & $\begin{array}{l}\text { Norm }_{2} \\
11_{-6}^{+10} \%\end{array}$ & $\begin{array}{c}N_{\mathrm{H} 2} \\
20_{-4}^{+5} \%\end{array}$ & 4 \\
\hline IC $2560(\mathrm{X}, \mathrm{C})^{*}$ & - & $\begin{array}{c}40.57 \\
0 \%\end{array}$ & $\begin{array}{c}41.03 \\
0 \%\end{array}$ & 6.46 & -2.02 & $\begin{array}{l}\text { 2ME2PL } \\
(+1 \text { gauss })\end{array}$ & -6 & - & 0.16 \\
\hline NGC $3393(\mathrm{C})^{*}$ & - & $\begin{array}{c}41.64 \\
0 \%\end{array}$ & $\begin{array}{l}41.29 \\
0 \%\end{array}$ & 8.10 & -3.41 & 2ME2PL & - & - & 7 \\
\hline$(\mathrm{X}, \mathrm{C})$ & & $\begin{array}{c}41.44 \\
0 \%\end{array}$ & $\begin{array}{c}41.26 \\
0 \%\end{array}$ & & & 2ME2PL & - & - & 0.66 \\
\hline NGC 4507 (X) & HBLR & $\begin{array}{c}42.04 \\
96_{-}^{+} 4 \%\end{array}$ & $\begin{array}{c}42.67 \\
81_{-}^{+} 10 \%\end{array}$ & 8.26 & -2.28 & $\begin{array}{l}\text { 2ME2PL } \\
\text { (+2gauss) }\end{array}$ & $\begin{array}{l}\mathrm{Norm}_{2} \\
51_{-20}^{+26 \%}\end{array}$ & $\begin{array}{c}N_{\mathrm{H} 2} \\
4_{-9}^{+12} \%\end{array}$ & 9 \\
\hline$(\mathrm{X}, \mathrm{C})$ & & $\begin{array}{c}41.96 \\
45_{-}^{+} 3 \%\end{array}$ & $\begin{array}{c}42.85 \\
38_{-}^{+} 3 \%\end{array}$ & & & & $\begin{array}{l}\mathrm{Norm}_{2} \\
53_{-27}^{+36} \%\end{array}$ & - & 0.41 \\
\hline NGC $4698(\mathrm{X})$ & - & $\begin{array}{c}40.14 \\
0 \%\end{array}$ & $\begin{array}{c}40.08 \\
0 \%\end{array}$ & 7.53 & -4.04 & $2 \mathrm{PL}$ & - & - & 9 \\
\hline NGC $5194(\mathrm{C})^{*}$ & NHBLR & $\begin{array}{c}39.53 \\
0 \%\end{array}$ & $\begin{array}{l}39.51 \\
0 \%\end{array}$ & 6.73 & -3.82 & ME2PL & - & - & 11 \\
\hline$(\mathrm{X}, \mathrm{C})$ & & $\begin{array}{c}39.94 \\
0 \%\end{array}$ & $\begin{array}{c}39.39 \\
0 \%\end{array}$ & & & 2ME2PL & - & - & 0.6 \\
\hline MARK 268 (X) & - & $\begin{array}{c}41.34 \\
0 \%\end{array}$ & $\begin{array}{c}42.92 \\
0 \%\end{array}$ & 7.95 & -1.62 & ME2PL & - & - & 0.01 \\
\hline MARK $273(\mathrm{X}, \mathrm{C})^{C L ?}$ & - & $\begin{array}{c}41.34 \\
24+2 \%\end{array}$ & $\begin{array}{c}42.29 \\
32+6 \%\end{array}$ & 7.74 & -2.05 & 2ME2PL & $\begin{array}{c}N_{\mathrm{H} 2} \\
51_{-14}^{+15} \%\end{array}$ & - & 2 \\
\hline Circinus $(\mathrm{C})^{*}$ & HBLR & $\begin{array}{c}39.80 \\
0 \%\end{array}$ & $\begin{array}{c}40.60 \\
0 \%\end{array}$ & 7.71 & -3.71 & $\begin{array}{l}\text { 2ME2PL } \\
\text { (+4gauss) }\end{array}$ & -14 & - & 9 \\
\hline NGC $5643(\mathrm{X})^{*}$ & NHBLR & $\begin{array}{c}40.44 \\
0 \%\end{array}$ & $\begin{array}{l}40.87 \\
0 \%\end{array}$ & 6.30 & -2.02 & 2ME2PL & - & - & 6 \\
\hline MARK $477(\mathrm{X})^{*}$ & HBLR & $\begin{array}{c}42.60 \\
0 \%\end{array}$ & $\begin{array}{c}43.11 \\
0 \%\end{array}$ & 7.20 & -0.68 & 2ME2PL & - & - & 0.01 \\
\hline IC 4518A (X) & - & $\begin{array}{c}42.06 \\
40_{-}^{+} 2 \%\end{array}$ & $\begin{array}{c}42.45 \\
41_{-}^{+} 6 \%\end{array}$ & 7.48 & -1.63 & 2ME2PL & $\begin{array}{l}\mathrm{Norm}_{2} \\
42_{-30}^{+45} \%\end{array}$ & - & 0.02 \\
\hline ESO 138-G01 (X)* & - & $\begin{array}{c}42.23 \\
0 \%\end{array}$ & $\begin{array}{c}42.11 \\
0 \%\end{array}$ & 5.50 & 0.01 & ME2PL & -30 & - & 6 \\
\hline
\end{tabular}

Notes. Column 1: name (the asterisks represent Compton-thick or changing look candidates), and the instrument (C: Chandra and/or X: XMM-Newton) in parenthesis; Col. 2: (non) hidden broad line region objects only in the cases where there are available observations; Cols. 3 and 4: logarithm of the soft $(0.5-2 \mathrm{keV})$ and hard $(2-10 \mathrm{keV}) \mathrm{X}$-ray luminosities, where the mean was calculated for variable objects, and percentages in flux variations; Col. 5: black-hole mass on logarithmical scale, determined using the correlation between stellar velocity dispersion (from HyperLeda) and black-hole mass (Tremaine et al. 2002), or obtained from the literature otherwise (MARK 1210 and NGC 4507 from Nicastro et al. 2003; IC 4518A from Alonso-Herrero et al. 2013; NGC 6300 and NGC 5643 from Davis et al. 2014; IC 2560 from Baloković et al. 2014; MARK 268 from Khorunzhev et al. 2012; and MARK 477 from Singh et al. 2011); Col. 6: Eddington ratio, $L_{\text {bol }} / ! L_{\text {Edd }}$, calculated from Eracleous et al. (2010) using $L_{\mathrm{bol}}=33 L_{2-10 \mathrm{kev}}$; Col. 7: best fit for SMF0; Col. 8: parameter varying in SMF1, with the percentage of variation; Col. 9: parameter varying in SMF2, with the percentage of variation; Col. 10: and the sampling timescale, corresponding to the difference between the first and the last observation. The percentages correspond to this $\Delta T_{\max }$.

$$
\begin{aligned}
& \text { Norm }_{1}=70.22_{58.82}^{81.21} \times 10^{-4} \text { Photons } \mathrm{keV}^{-1} \mathrm{~cm}^{-2} \mathrm{~s}^{-1} \text {, } \\
& N_{\mathrm{H} 2}=9.68_{8.20}^{11.31} \times 10^{22} \mathrm{~cm}^{-2}, \quad \Gamma=1.30_{1.07}^{1.54} \text {, } \\
& \text { Norm }_{\text {pex }}=0.10_{0.07}^{0.15} \times 10^{-4} \text { Photons } \mathrm{keV}^{-1} \mathrm{~cm}^{-2} \mathrm{~s}^{-1} \text {, } \\
& \text { Norm }_{2}=5.51_{3.55}^{8.72} \times 10^{-4} \text { Photons keV } \mathrm{cm}^{-1} \mathrm{~s}^{-1}
\end{aligned}
$$

(XMM-Newton obsID. $\quad 0064600101), \quad 3.03_{1.93}^{4.86} \times$ $10^{-4} \quad$ Photons $\mathrm{keV}^{-1} \quad \mathrm{~cm}^{-2} \mathrm{~s}^{-1} \quad$ (XMM-Newton obsID. 0064600301), and $\chi /$ d.o.f. $=109.30 / 126$. Thus, the best representation of the data requires $\mathrm{Norm}_{2}$ to 
Table 2. continued.

\begin{tabular}{|c|c|c|c|c|c|c|c|c|c|}
\hline \multirow{2}{*}{$\begin{array}{l}\text { Name } \\
\text { (1) }\end{array}$} & \multirow{2}{*}{$\begin{array}{l}\text { Type } \\
\text { (2) } \\
\end{array}$} & \multirow{2}{*}{$\begin{array}{c}\log \left(L_{\text {soft }}\right) \\
(0.5-2 \mathrm{keV}) \\
(3)\end{array}$} & \multirow{2}{*}{$\begin{array}{c}\log \left(L_{\text {hard }}\right) \\
(2-10 \mathrm{keV}) \\
(4)\end{array}$} & \multirow{2}{*}{$\begin{array}{c}\log \left(M_{\mathrm{BH}}\right) \\
(5)\end{array}$} & \multirow{2}{*}{$\begin{array}{c}\log \left(R_{\text {Edd }}\right) \\
(6) \\
\end{array}$} & \multicolumn{3}{|c|}{ Variability } & \multirow{2}{*}{$\begin{array}{c}\Delta T_{\max } \\
\text { (Years) } \\
(10) \\
\end{array}$} \\
\hline & & & & & & $\begin{array}{l}\text { SMF0 } \\
(7)\end{array}$ & $\begin{array}{c}\text { SMF1 } \\
(8)\end{array}$ & $\begin{array}{c}\text { SMF2 } \\
(9)\end{array}$ & \\
\hline NGC 6300 (C) & - & $\begin{array}{c}41.32 \\
0 \%\end{array}$ & $\begin{array}{c}41.95 \\
0 \%\end{array}$ & 7.18 & -2.68 & $2 \mathrm{PL}$ & - & - & 0.01 \\
\hline$(\mathrm{X}, \mathrm{C})$ & & $\begin{array}{c}41.06 \\
98_{-}^{+} 50 \%\end{array}$ & $\begin{array}{c}41.68 \\
98_{-}^{+} 16 \%\end{array}$ & & & $2 \mathrm{PL}$ & $\begin{array}{l}\mathrm{Norm}_{2} \\
98^{+12} \%\end{array}$ & $\begin{array}{l}\text { Norm }_{1} \\
93^{+25} \%\end{array}$ & 8 \\
\hline NGC $7172(\mathrm{X})$ & NHBLR & $\begin{array}{c}42.50 \\
51_{-}^{+} 2 \%\end{array}$ & $\begin{array}{c}42.82 \\
51_{-}^{+} 1 \%\end{array}$ & 8.20 & -1.98 & ME2PL & $\begin{array}{l}\text { Norm }_{2} \\
51_{-5}^{+5} \%\end{array}$ & - & 5 \\
\hline NGC $7212(\mathrm{X}, \mathrm{C})^{*}$ & HBLR & $\begin{array}{c}41.81 \\
0 \%\end{array}$ & $\begin{array}{c}42.60 \\
0 \%\end{array}$ & 7.54 & -1.55 & 2ME2PL & $\begin{array}{l}-5 \\
-\end{array}$ & - & 1 \\
\hline $\operatorname{NGC} 7319(\mathrm{C})^{C L ?}$ & - & $\begin{array}{c}42.99 \\
38_{-}^{+} 8 \%\end{array}$ & $\begin{array}{c}42.98 \\
38_{-}^{+} 5 \%\end{array}$ & 7.43 & -1.26 & ME2PL & $\begin{array}{l}\mathrm{Norm}_{2} \\
39_{-22}^{+53} \%\end{array}$ & $\begin{array}{c}N_{\mathrm{H} 1} \\
100_{-23}^{+27} \%\end{array}$ & 7 \\
\hline$(\mathrm{X}, \mathrm{C})$ & & $\begin{array}{c}42.58 \\
71_{-}^{+} 8 \%\end{array}$ & $\begin{array}{c}42.84 \\
69_{-}^{+} 7 \%\end{array}$ & & & ME2PL & $\begin{array}{l}\mathrm{Norm}_{2} \\
72_{-46}^{+64 \%} \%\end{array}$ & - & 6 \\
\hline
\end{tabular}
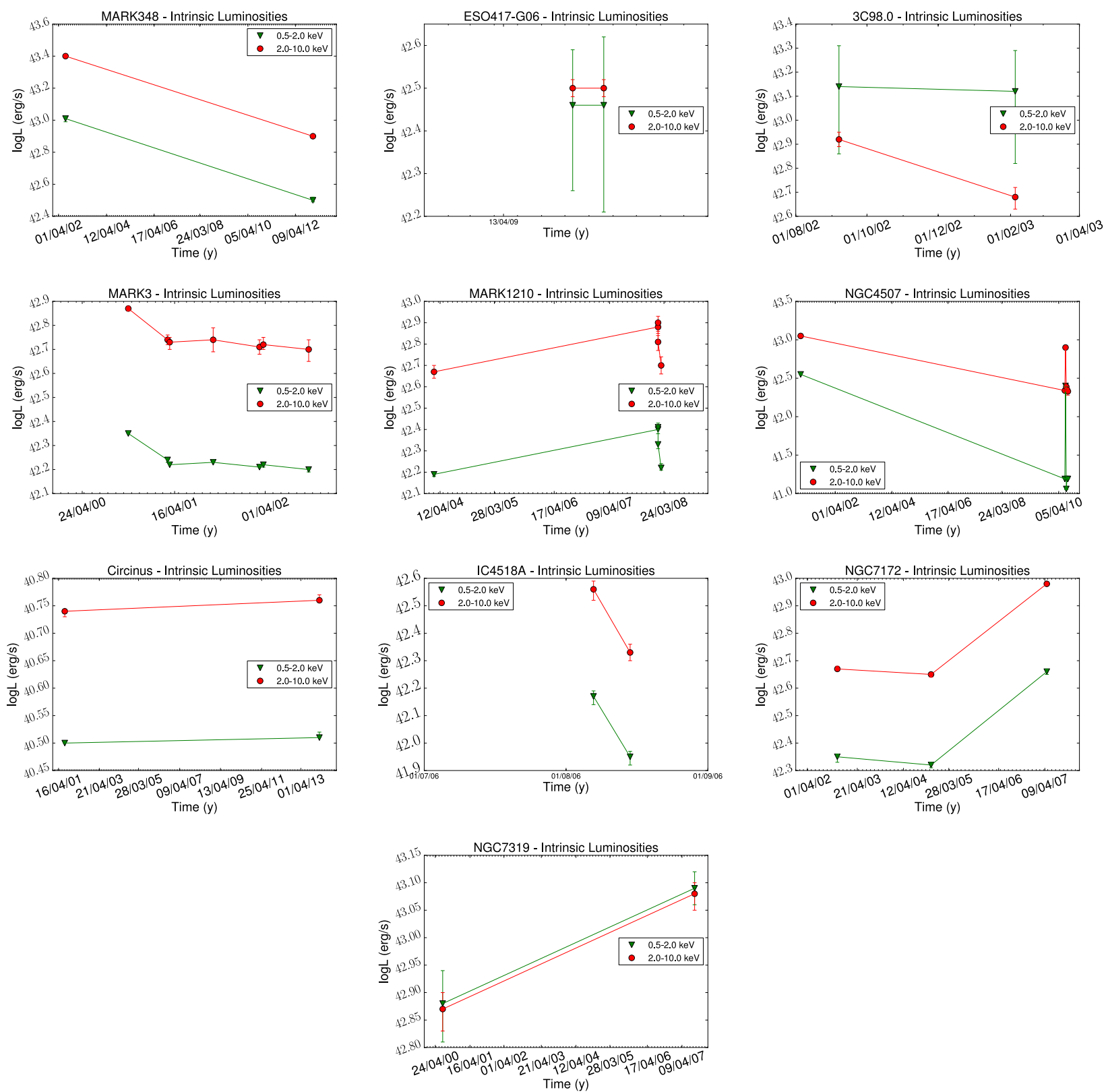

Fig. 3. X-ray intrinsic luminosities calculated for the soft $(0.5-2.0 \mathrm{keV}$, green triangles) and hard (2.0-10.0 keV, red circles) energies in the simultaneous fits, only for the variable objects. 
vary between the two XMM-Newton data sets, while the reflection component remains constant. This spectral fit with $\mathrm{Norm}_{2}$ varying agrees with the one using the MEPL model (Table A.2). The percentages of the variations are compatible between the two SMF1 and also the luminosities. For simplicity, we report the results of the MEPL model in the following. The simultaneous fit of the XMM-Newton data needs SMF1 with Norm $_{2}$ (43\%) varing over a period of about half a year. This implies an intrinsic flux variation of 5\% (42\%) at soft (hard) energies. The annular region contributes with $8 \%$ to the Chandra data, and SMF0 was used when comparing Chandra and $X M M-N e w t o n$ data, i.e., variations were not found within a five-year period. Short-term variations are not detected from the Chandra data. UV data from the UVW1 filter did not show any variability. We classify it as a Compton-thin candidate.

- MARK 3: the XMM-Newton data need SMF1 with Norm $_{2}$ $(37 \%)$ as the parameter responsible for the variations. This corresponds to flux variations of $29 \%(32 \%)$ in the soft (hard) energy band in a one-year period. We classify it as a Compton-thick candidate.

- MARK 1210: X-rays observations with Chandra covering a period of about four years are simultaneously fitted, resulting in SMF2 with $\mathrm{N}_{\mathrm{H} 2}(20 \%)$ and $\mathrm{Norm}_{2}(43 \%)$ as the parameters varying in this model. This corresponds to intrinsic flux variations of $40 \%(41 \%)$ at soft (hard) energies. We classify the object as a Compton-thin candidate.

- NGC 3079: one observation per instrument is available. The annular region contributes with $79 \%$ to Chandra data. The estimated number counts in the nuclear component of the XMM-Newton spectrum is 235 counts, so we do not perform a simultaneous fitting. This object will not be used to discuss long-term variations. We classify it as a Comptonthin candidate. We refer the reader to Appendix B for the discussion of this source.

- IC 2560: only one observation per instrument is available. When comparing the data, the annular region contributes with $11 \%$ to the Chandra data. No variations were observed within two months, i.e., SMF0 was used for the simultaneous fit. An additional Gaussian line was needed in the fit at $1.85 \mathrm{keV}$ (Si XIII). A XMM-Newton and a Chandra light curve were analyzed. We notice that the XMM-Newton light curve showed a positive value of $\sigma_{\mathrm{NXS}}^{2}$ at $2.5 \sigma$ of confidence level, close to our limit (see Sect. 4.4). We classify it as a Compton-thick candidate.

- NGC 3393: Chandra data are fitted with SMF0, resulting in no variations in a seven years period. When comparing with $X M M-N e w t o n$ data, the annular region contributes with $17 \%$, and SMF0 is needed to fit the data within a one-year period. Short-term variations are not found from one Chandra light curve. We classify it as a Compton-thick candidate.

- NGC 4507: SMF2 was used to fit the XMM-Newton data, with Norm $_{2}(36 \%)$ and $N_{\mathrm{H} 2}(21 \%)$ varying in a nine-year period. This corresponds to a flux variation of $96 \%(81 \%)$ in the soft (hard) energy band. Two additional Gaussian lines at $1.36(\mathrm{Mg} \mathrm{XI})$ and 1.85 ( $\mathrm{Si} \mathrm{XIII}) \mathrm{keV}$ are needed to fit the data. The annular region contributes with $13 \%$ to the Chandra data. When comparing Chandra and XMM-Newton data, the best fit resulted in SMF1 with Norm $_{2}$ (53\%) varying over nine years. Short-term variations are found from neither Chandra nor XMM-Newton light curves. We classify it as a Compton-thin candidate.
- NGC 4698: SMF0 was used in the simultaneous fit, resulting in no variations in a nine-year period. UV data in the UVM2 filter is available, where the object does not show changes. We classify it as a Compton-thin candidate.

- NGC 5194: the simultaneous fit results in no variations (i.e., SMF0 was used) within an 11-year period. The annular region contributes with $91 \%$ to the Chandra data. When comparing data from XMM-Newton and Chandra, SMF0 results in the best representation of the data. Six Chandra light curves were analyzed in three energy bands, but variations are not reported. UV data are available in three filters, one showing variations (UVW1) and the remaining two not (UVW2, UVM2). We classify it as a Compton-thick candidate.

- MARK 268: the XMM-Newton observations are separated by two days. SMF0 was used to fit the data. UV data are available in two filters (UVW1 and UVM2); none of them show variability. We classify it as a Compton-thin candidate.

- MARK 273: only one observation per instrument can be used for the variability analysis. The annular region contributes with $31 \%$ to the Chandra data. Variations in $N_{\mathrm{H} 2}(51 \%)$ were needed in the SMF1. This corresponds to a luminosity variation of $24 \%(32 \%)$ in the soft (hard) energy band over a two-year period. UV data are available in two epochs, with no variations observed. The analysis of the Chandra light curve results in no short-term variations. Compton-thick and Compton-thin classifications were obtained for different observations, so we classify it as a changing-look candidate (see Table A.7).

- Circinus: Chandra and XMM-Newton data are available at different epochs. The Chandra data analysis results in SMF0 (i.e., no variations) in a nine-year period, while the XMM-Newton data set needs SMF2 with Norm $_{1}$ (34\%) and $\mathrm{Norm}_{2}$ (31\%) varying within a 13-year period. However, the XMM-Newton data did not show any flux variations. The spectra are quite complex, so two (at 1.85 (Si XIII) and 2.4 (S XIV) $\mathrm{keV}$ ) and four (at 1.2 (Ne X), 1.36 (Mg XI), 1.85 (Si XIII), and 2.4 (S XIV) keV) additional Gaussian lines are required for the XMM-Newton and Chandra fits, respectively. The annular region contributes with $28 \%$ to the Chandra data. However, the comparison between the data sets was not carried out owing to the complexity of the spectra. Short-term variations are not found from a Chandra light curve. We classify it as a Compton-thick candidate. We notice that the variations obtained from XMM-Newton data will not be used for further discussion, because this variability seems to be caused by extranuclear sources (see B.18 for details), and therefore this nucleus is considered as nonvariable.

- NGC 5643: the XMM-Newton data were fitted with the SMF0; i.e., variations were not observed within a six-year period. We classify it as a Compton-thick candidate.

- MARK 477: the two observations are separated by two days. SMF0 was used, so no variations are reported. At UV frequencies variations are not found. We classify the source as a Compton-thick candidate.

- IC 4518A: the XMM-Newton data need SMF1 with Norm $_{2}$ (42\%) varying. The variations are found in an eight-day period, and correspond to a flux variation of $40 \%$ (41\%) in the soft (hard) energy band. We classify it as a Compton-thin candidate.

- ESO 138-G01: no variations are found (i.e., SMF0 was used) within a five-year period. We classify it as a Compton-thick candidate. 
- NGC 6300: The Chandra observations are separated by four days. SMF0 results in the best fit; i.e., variations are not found. The annular region contributes with $5 \%$ to the Chandra data. When comparing Chandra and XMM-Newton data, SMF2 was used, with Norm $_{1}$ (98\%) and Norm 2 (98\%) varying over an eight-year period. We classify it as a Compton-thin candidate.

- NGC 7172: SMF1 is the best representation of the XMM-Newton data, with $\mathrm{Norm}_{2}(54 \%)$ varying over a threeyear period. This implies an intrinsic flux variation of 54\% $(53 \%)$ at soft (hard) energies. We classify it as a Comptonthin candidate.

- NGC 7212: One observation per instrument is available. The annular region contributes with $16 \%$ to the Chandra data. When comparing both data sets, SMF0 is needed; i.e., variations are not found. We classify this source as a Comptonthick candidate.

- NGC 7319: the best representation of the data used SMF2 with $N_{\mathrm{H} 1}$ (passed from $N_{\mathrm{H} 1}=6.5 \times 10^{21} \mathrm{~cm}^{-2}$ to $N_{\mathrm{H} 1}=N_{\mathrm{Gal}}$ ) and $\mathrm{Norm}_{2}(39 \%)$ varying in a seven-year period. Intrinsic flux variations of $38 \%$ in both the soft and hard energy bands are obtained. The annular region contributes with $17 \%$ to the Chandra data. When comparing XMM-Newton and Chandra data, SMF1 with Norm $_{2}$ (54\%) varying is required, implying flux variations of $71 \%$ (69\%) at soft (hard) energies over six years. Short-term variations were not detected. We classify it as a changing-look candidate because Compton-thick and Compton-thin classifications were obtained for different observations (see Table A.7).

\subsection{Spectral characteristics}

The sample of 26 optically classified Seyfert 2 galaxies presented in this work show a variety of spectral shapes. None of them are well-fitted with the ME or the PL models alone. Composite models are required in all cases.

The models we used in previous works (to represent the spectra of LINERs, González-Martín et al. 2009b; Hernández-García et al. 2013, 2014) describe the spectra of 12 galaxies well (MARK 348, ESO 417-G06, MARK 1066, 3C 98.0, NGC 3079, NGC 4698, NGC 5194, MARK 268, ESO 138-G01, NGC 6300, NGC 7172, and NGC 7319). Three models are required (2PL, MEPL, and ME2PL) for the spectral fits. Among the 15 objects in our sample observed in polarized light (see Table 1), one galaxy in this group has a HBLR and four a NHBLR.

On the other hand, 14 objects (NGC 424, MARK 573, NGC 788, MARK 3, MARK 1210, IC 2560, NGC 3393, NGC 4507, MARK 273, Circinus, NGC 5643, MARK 477, IC 4518A, and NGC 7212) show a more complex structure at energies below and around $2 \mathrm{keV}$, which cannot be fitted with a single thermal component. These nuclei need the 2ME2PL model to fit the data. Besides, four of the objects need additional Gaussian lines to properly fit the data. Nine galaxies in this group have a HBLR and one a NHBLR.

The addition of a cold reflection component to the best-fit model is not statistically required by the data, except in obsID 0064600101 (XMM-Newton) of 3C 98.0. It is worth noting that even if a model including this component is physically more meaningful, the lack of data at harder energies prevents us from setting the best values required by the model, and therefore a single power law is enough for studying nuclear variations. On the other hand, we find that the cold reflection component remains constant for 3C 98.0 in SMF1. If this is the general scenario (see
Sect. 6.2), the lack of this component in the models will not introduce biases into the variability analysis.

A thermal component at soft energies is needed to fit the data in 24 out of the 26 sources; in 14 cases, two MEKAL are needed. It is worth recalling that even if a MEKAL model fits the data well, because of its spectral resolution, photoionized models would be required to properly describe the data (see Sect. 4.1). The values of the temperatures are in the range $k T_{1}=[0.04-0.26] \mathrm{keV}$ (only when the 2ME2PL model is fitted) with a mean value of $0.12_{-}^{+} 0.03 \mathrm{keV}$, and $k T_{2}=[0.13-1.00] \mathrm{keV}$ with a mean value of $0.60_{-}^{+} 0.14 \mathrm{keV}$. The values of the spectral index (which is the same at soft and hard energies, when two are required) is in the range $\Gamma=[0.61-3.23]$, with a mean value of $1.56_{-}^{+} 0.40$, and the absorbing column densities at hard energies $N_{\mathrm{H} 2}=[5.15-152.21] \times 10^{22} \mathrm{~cm}^{-2}$, with a mean value of $34.69_{-}^{+} 15.30 \times 10^{22} \mathrm{~cm}^{-2}$.

\subsection{Long-term $X$-ray spectral variability}

From the 26 galaxies in our sample, we compared data at different epochs from the same instrument in 19 cases. Among these, seven objects were observed with Chandra, 13 with $X M M-N e w t o n$, and in one case (namely Circinus) observations at different epochs with both instruments were available.

Chandra and XMM-Newton data are available for the same object in 15 cases (see Table A.1). We did not compare these data sets for NGC 3079 because the number counts of the nuclear contribution of XMM-Newton spectrum (after decontaminating from the annular region) is not enough for a reliable spectral fit. Given that NGC 3079 has one observation per instrument that cannot be compared, this object will not be used to discuss longterm variations. Additionally, the Chandra and XMM-Newton spectra of Circinus are very different, most probably because extranuclear sources are included in the XMM-Newton aperture radius, thus preventing us from properly comparing both. For the remaining 13 objects, the simultaneous analysis was carried out (Table A.5), where the extranuclear emission were negligible in two cases (NGC 424 and NGC 788). Four of these sources showed spectral variations.

In total, 25 (out of 26) nuclei have been analyzed to study long-term X-ray spectral variations, with 11 of them (excluding Circinus $^{9}$ ) showing variability. In Fig. 4 (left) we present a histogram of the luminosities of the variable and non-variable sources. A K-S test results in $p=0.006$, so we can reject the hypothesis that the sample came from the same normal distribution. The spectral changes are mainly due to variations in the nuclear power (i.e., Norm $_{2}$ ), which is observed in nine nuclei (MARK 348, 3C 98.0, MARK 3, MARK 1210, NGC 4507, IC 4518A, NGC 6300, NGC 7172, and NGC 7319). Changes in the column density (i.e., $N_{\mathrm{H} 2}$ ) are also present in four cases (ESO 417-G06, MARK 273, MARK 1210, and NGC 4507 - in the last two accompained by changes in Norm $_{2}$ ). Changes at soft energies are found in two objects: NGC $7319\left(N_{\mathrm{H} 1}\right.$ together with $\left.\mathrm{Norm}_{2}\right)$ and NGC $6300\left(\mathrm{Norm}_{1}\right.$ together with $\left.\mathrm{Norm}_{2}\right)$. This means that from the 11 sources showing variations, most of them (nine out of 11) show variations in the nuclear continuum (i.e., $\mathrm{Norm}_{2}$ ), while variations due to absorptions are less common (four in total, in two objects accompained by variations in $\left.\mathrm{Norm}_{2}\right)$.

9 We exclude the variations found with XMM-Newton data because they are most probably due to extranuclear sources, while variations with Chandra data are not reported. 

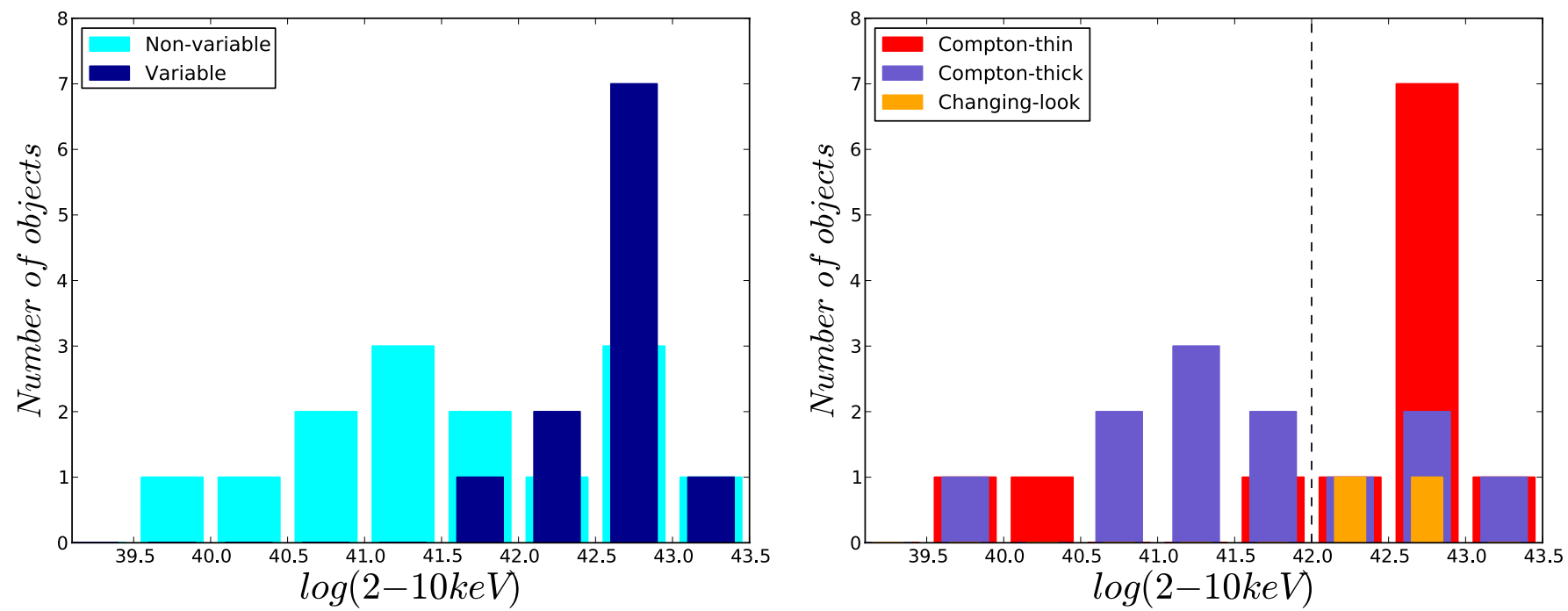

Fig. 4. Histograms of: left: the luminosities for the variable (dark blue) and non-variable (light blue) galaxies in the sample; and right: the luminosities for the galaxies in the sample divided into Compton-thick (purple), Compton-thin (red), and changing-look (orange) candidates. The dashed line represents the value for the selection of faint (below) and bright (above) Seyfert 2s.

Table 3. Mean values of the spectral parameters for the subgroups.

\begin{tabular}{l|ccc}
\hline \hline Group & $\Gamma$ & $N_{\mathrm{H} 2}$ & $\log (L(2-10 \mathrm{keV}))$ \\
\hline All & $1.56_{-}^{+} 0.40$ & $34.69_{-}^{+} 15.30$ & $42.56_{-}^{+} 0.89$ \\
HBLR & $1.34_{-}^{+} 0.43$ & $39.22_{-}^{+} 18.62$ & $42.72_{-}^{+} 0.80$ \\
NHBLR & $1.58_{-}^{+} 0.48$ & $40.17_{-}^{+} 20.23$ & $41.40_{-}^{+} 1.04$ \\
Compton-thick & $0.57_{-}^{+} 0.29^{1}$ & $43.95_{-}^{+} 19.53$ & $42.33_{-}^{+} 1.01$ \\
Compton-thin & $1.43_{-}^{+} 0.32$ & $20.31_{-}^{+} 14.39$ & $42.73_{-}^{+} 1.12$ \\
Changing-look & $1.68_{-}^{+} 0.49$ & $45.99_{-}^{+} 1.24$ & $42.76_{-}^{+} 0.49$ \\
Bright & $1.44_{-}^{+} 0.40$ & $32.11_{-}^{+} 20.12$ & $42.78_{-}^{+} 0.29$ \\
Faint & $1.69_{-}^{+} 0.61$ & $34.53_{-}^{+} 21.20$ & $41.38_{-}^{+} 0.82$ \\
\hline
\end{tabular}

Notes. Col. 1: Group; Col. 2: values of $\Gamma$; Col. 3: column density in units of $10^{22} \mathrm{~cm}^{-2}$; and Col. 4: intrinsic luminosity in the $2-10 \mathrm{keV}$ energy band. ${ }^{(1)}$ This value is calculated from the simultaneous values reported in Table A.7.

\subsubsection{HBLR vs. NHBLR}

From the 15 objects in the sample with available observations in polarized light (see Table 1), ten are HBLR objects and five NHBLR. Nine out of the ten HBLR objects need the 2ME2PL model for the spectral fits (except MARK 348). The mean values of the parameters in the simultaneous fits are reported in Table 3. From the ten HBLR, four (MARK 348, MARK 3, MARK 1210, and NGC 4507) show variations in $\mathrm{Norm}_{2}$, in two sources accompained by variations in $N_{\mathrm{H} 2}$. One (NGC 7172) out of the four NHBLR sources shows variations in $\mathrm{Norm}_{2}$.

Therefore, although the number of objects in this subsample is not enough to be conclusive, it seems that there is no difference in either the proportion of variable objects or in the pattern of the variations.

\subsubsection{Compton-thick vs. Compton-thin}

We select Compton-thick candidates when at least two out of the three indicators were met (see Sect. 4.5). These indicators are obtained from X-ray $(\mathrm{EW}(\mathrm{FeK} \alpha)$ and $\Gamma)$ and the [O III] line $\left(F_{\mathrm{x}} / F_{[\mathrm{OIII}]}\right)$ data. In Fig. 5 we represent the histogram of these values for the whole sample, where the mean was calculated when multiple observations were available (from
Table A.7). One Compton-thin candidate has $\Gamma<1$ (NGC 4698), one Compton-thick candidate has $E W(\mathrm{FeK} \alpha)<0.5 \mathrm{keV}$ (MARK 477), one Compton-thin candidate has $\log \left(F_{\mathrm{X}} / F_{[\mathrm{OIII}]}\right)<$ 0 (NGC 3079), and four Compton-thick candidates have $\log \left(F_{\mathrm{x}} / F_{[\mathrm{OIII}]}\right)>0$ (NGC 424, IC 2560, ESO 138-G01, and NGC 7212; see discussion in Sect. 6.2).

From the 26 nuclei, 12 are classified as Compton-thick candidates (NGC 424, MARK 573, MARK 3, MARK 1066, IC 2560, NGC 3393, NGC 5194, Circinus, NGC 5643, MARK 477, ESO 138-G01, and NGC 7212), 12 as Comptonthin candidates (MARK 348, NGC 788, ESO 417-G06, 3C 98.0, MARK 1210, NGC 3079, NGC 4507, NGC 4698, MARK 268, IC 4518A, NGC 6300, and NGC 7172), and two as changinglook candidates (MARK 273, and NGC 7319). The mean values of the spectral parameters in these subgroups are reported in Table 3, where Compton-thin candidates are more luminous and less obscured and have steeper spectral indices than Compton-thick candidates. The spectral index of Comptonthick candidates was estimated using $\Gamma_{\text {soft }} \neq \Gamma_{\text {hard }}$ (see details in Sect. 4.5) and the values are reported in Table A.7.

Only one (out of the 12) Compton-thick candidates shows variations (MARK 3), in $\mathrm{Norm}_{2}$. Eight (out of 11) Compton-thin candidates show changes, with these variations related mainly to $\mathrm{Norm}_{2}$ (seven cases, in three sources accompained by variations in $N_{\mathrm{H} 2}$ or Norm $_{1}$ ) and only in one case to $N_{\mathrm{H} 2}$ alone. The two changing-look candidates show $\mathrm{X}$-ray long-term variations, MARK 273 varies $N_{\mathrm{H} 2}$, and NGC 7319 needs variations in $N_{\mathrm{H} 1}$ plus $\mathrm{Norm}_{2}$.

Therefore, the number of variable Compton-thin and changing look candidates is notably higher than that of Compton-thick candidates.

\subsubsection{Bright vs. faint nuclei}

In Fig. 4 (right), we present the histogram of the luminositites of the AGN in the sample as reported in Table 2, for Comptonthick (purple), Compton-thin (red), and changing-look (orange) candidates. A bimodal distribution can be appreciatted (K-S test, $p=0.030)$, with the difference around $\log (L(2-10 \mathrm{keV})) \sim 42$. Based on this histogram we separate the objects into faint (with 
$\log (L(2-10 \mathrm{keV}))<42)$ and bright $(\log (L(2-10 \mathrm{keV}))>42)$ Seyfert $2 \mathrm{~s}$.

From these, 15 sources are bright, including four Comptonthick (one variable, MARK 3), two changing-look (both variable, MARK 273, and NGC 7319), and nine Comptonthin (seven variable, MARK 348, ESO 417-G06, 3C 98.0, MARK 1210, NGC 4507, IC 4518A, and NGC 7172). The remaining 11 objects are faint Seyfert $2 \mathrm{~s}$, including three Compton-thin (one shows variations, NGC 6300) and eight Compton-thick (none varies).

In total, 10 (out of 15) bright nuclei, and one (out of 10) faint nuclei show variations. Therefore, brighter sources include more variable sources and less Compton-thick candidates, a trend that can be derived by comparing left- and righthand panels in Fig. 4 . Moreover, we note that NGC 6300 (i.e., the only faint source that varies) has $\log (L(2-10 \mathrm{keV}))=41.95$, very close to the established luminosity limit. The mean values of the spectral parameters of these subgroups are reported in Table 3, where faint objects show a steeper power law index than bright objects.

\subsection{Short-term X-ray variability}

Observations with a net exposure time $>30 \mathrm{ks}$ are used to study short-term variations. This requirement leaves us with ten sources for the analysis (see Table A.6). Three of them (IC 2560, NGC 5194, and MARK 573) show positive values of $\sigma_{\mathrm{NXS}}^{2}$, but below $3 \sigma$ of confidence level in all cases. Therefore we cannot claim short-term variations in any of the objects in our sample. Upper limits of $\sigma_{\mathrm{NXS}}^{2}$ have been estimated for all the other cases.

\subsection{Long-term UV flux variability}

$X M M$-Newton data at different epochs were used to study longterm X-ray spectral variations in 13 sources. In nine of them data from the OM cannot be used because the source is outside the detector or because the same filter is not available at different epochs. In contrast, two objects (MARK 273 and NGC 5194) have OM data while the sources were out of the pn detector, so these data were also used to search for variations at UV frequencies. Thus, UV data for variability studies are available for six galaxies (3C 98.0, NGC 4698, NGC 5194, MARK 268, MARK 273, and MARK 477). Only NGC 5194 shows variations above $3 \sigma$ of the confidence level in one filter (UVW1).

We also searched in the literature for UV variations for the sources in the sample, but this information was available only for MARK 477 (see Appendix B). Comparing the analyses at $\mathrm{X}$-rays and UV, two out of the six sources do vary at X-rays but not at UV frequencies (3C 98.0 and MARK 273), and one (NGC 5194) does not show variations in X-rays but it does at UV. The remaining three objects do not vary neither in X-rays nor at UV frequencies.

\section{Discussion}

\subsection{X-ray spectral variability}

A long-term X-ray variability analysis was performed for 25 out of the 26 nuclei in our sample of Seyfert 2 galaxies ${ }^{10}$. From these, 11 sources are variable at X-rays. Among the remaining 14 nuclei where variations are not detected, 11 are Comptonthick candidates, and therefore variations are not expected (e.g.,

\footnotetext{
${ }^{10}$ We recall that NGC 3079 will not be used for the discussion of variability, see Sect. 5.1.
}

Matt et al. 2013, and references therein). This agrees well with our results, where only one out of the 12 Compton-thick candidates shows variations. We refer the reader to Sect. 6.2 for a complete discussion about Compton-thick candidates. The other three nuclei where variations are not detected are Compton-thin candidates (NGC 788, NGC 4698, and MARK 268). The lack of variations may be due to the short timescale between observations for MARK 268 (two days). The timescales between observations for the other two sources are on the order of years, so, in principle, variations could be detected. New data would therefore be required before confirming the non-variable nature of these sources.

In this section the discussion is focused on the different patterns of variability obtained for the 11 variable nuclei, including eight Compton-thin, two changing-look, and one Comptonthick candidates. We notice that this is the first time that transitions from a Compton-thin to a Compton-thick (or vice versa) appearance have been reported for MARK 273 and NGC 7319, which should be added to the short list of known changing-look Seyfert 2s, such as NGC 2992 (Gilli et al. 2000), MARK 1210 (Guainazzi et al. 2002), NGC 6300 (Guainazzi 2002), NGC 7674 (Bianchi et al. 2005a), and NGC 7582 (Bianchi et al. 2009).

\subsubsection{Variations at soft energies}

We found that most of the objects in our sample do not vary at soft X-ray energies, indicating that the mechanism responsible for the soft emission should be located far from the nucleus. Indeed, using artificial neural networks, González-Martín et al. (2014) compared the spectra of different classes of AGN and starburst galaxies and find that Seyferts 2 have a high contribution from processes that are related to star formation, which may be related to emission coming from the host galaxy.

Notwithstanding, two sources show variations at soft energies $(<2 \mathrm{keV})$, each showing a different variability pattern, but in both cases these variations are accompanied by variations in the normalization of the hard power law; NGC 6300 shows variations in the normalization at soft energies, $\mathrm{Norm}_{1}$, when comparing data from XMM-Newton and Chandra; and NGC 7319 showed variations in the absorber at soft energies, $N_{\mathrm{H} 1}$, when comparing two Chandra observations. It is worth noting that the soft X-ray fluxes are on the order of $10^{-13} \mathrm{erg} \mathrm{cm}^{-2} \mathrm{~s}^{-1}$ in the two nuclei, which is typical of Seyfert galaxies (Guainazzi et al. 2005 b), so these variations are not related to low-count number statistics. However, variations at soft energies in these sources have not been reported before. Up to now, such variations have only been found for two Seyfert 2s. Paggi et al. (2012) found variations at soft X-rays in the Seyfert 2 MARK 573 when comparing four Chandra observations. This nucleus is also included in the present sample, but variations are not found here, mainly because we did not use two of the observations included in the work of Paggi et al. (2012) since they were affected by a pileup fraction higher than $10 \%$. Guainazzi et al. (2012) speculate that variations at soft X-ray energies in MARK 3 may be present when comparing XMM-Newton and Swift data, but confirmation is still required. They argue that these variations are most probably due to cross-calibration uncertainties between the instruments, but if true, soft X-ray variations could be related to the innermost part of the narrow-line region.

On the other hand, the variability patterns found in this work have also been reported for other types of AGN. Variations in the absorbers, as seen in NGC 7319, were found by GonzálezMartín et al. (2011a), who used Suzaku data to study the LINER 2 NGC 4102. They argue that the variations at soft energies are 
due to an absorbing material located within the torus and perpendicular to the plane of the disk. Variability timescales can be used to estimate the lower limits of the cloud velocity (e.g., Risaliti et al. 2007). However, the timescales between our observations were obtained randomly, so the variability timescale of the eclipse can be shorter. In the case of NGC 7319, variations are obtained within a timescale of seven years, which is too long to estimate the distance at which the cloud is located. It is worth noting that we classified this object as a changing-look candidate. Besides, we found that NGC 6300 varied the normalizations at soft and hard energies. Using the same method as explained in this work, Hernández-García et al. (2013) find the same variability pattern in the LINER 2 NGC 4552, indicating that these variations may be intrinsic to the emitting material.

\subsubsection{Absorber variations}

Variations in the circumnuclear absorbers are thought to be very common in Seyfert galaxies. In fact, these variations are usually observed in Seyferts 1-1.9 (e.g., NGC 1365, Risaliti et al. 2007; NGC 4151, Puccetti et al. 2007; MARK 766, Risaliti et al. 2011), where it has been shown that the changes are most probably related to the broad line region (BLR), although it has been suggested that multiple absorbers may be present in an AGN, located at different scales (Braito et al. 2013). However, it is not so clear whether variations due to absorbers are common for optically classified Seyfert $2 \mathrm{~s}$, for which this kind of variation has only been reported in a few cases (e.g., MARK 348, Marchese et al. 2014; NGC 4507, Braito et al. 2013 and Marinucci et al. 2013; MARK 1210, Risaliti et al. 2010).

From the 11 variable sources in our sample, variations due to absorbers at hard energies are detected in four nuclei. In two of them, MARK 1210 and NGC 4507, variations in $N_{\mathrm{H} 2}$ are accompained by variations in the nuclear continuum, $\mathrm{Norm}_{2}$. The variability pattern reported for these objects agrees with previous results presented by Risaliti et al. (2010) and Braito et al. (2013), who argue that the physical properties of the absorber are consistent with these variations occurring in the BLR. Following prescriptions in Risaliti et al. (2010) and using the BH masses (Table 2) and variability timescales of one and ten days for MARK 1210 and NGC 4507, respectively, we estimate the cloud velocities to be higher than $10^{3} \mathrm{~km} \mathrm{~s}^{-1}$ in both cases, thus also locating the absorbers at the BLR.

On the other hand, ESO 417-G06 and MARK 273 showed variations only in $N_{\mathrm{H} 2}$. Trippe et al. (2011) report variations of a factor about two in the count rate of ESO 417-G06 from the 22-month survey of Swift, and Balestra et al. (2005) fit the XMM-Newton and Chandra spectra of MARK 273 studied in this work and note that different column densities were required to fit the data well (its values in good agreement with ours), indicating variations due to absorption. The timescale between observations for ESO 417-G06 is 40 days and two years for MARK 273. Therefore, we cannot estimate the cloud velocity for MARK 273 because the timescale is too large. Assuming the variability timescale of ESO 417-G06 (40 days) and following prescriptions in Risaliti et al. (2010), we estimate a cloud velocity $>60 \mathrm{~km} \mathrm{~s}^{-1}$, so too low to restrict the location of the cloud. Since this estimate is a lower limit of the cloud velocity, a monitoring campaign of these sources would be needed to constrain their variability timescales, in order to properly constrain the locus of the absorbers.

\subsubsection{Flux variations}

The most frequently varying parameter in our sample is $\mathrm{Norm}_{2}$, which is related to the nuclear continuum. These kinds of variations are observed in nine out of the $11 \mathrm{X}$-ray variable sources sometimes accompanied by variations in other parameters (see Sects. 6.1.1 and 6.1.2). Therefore the most natural explanation for the observed variations in Seyfert 2 galaxies is that the nuclear power is changing with time. We recall that variations are not due to changes in the power law index, $\Gamma$, but related to its normalization. It has been shown that hard X-ray variability is usual in Seyfert 2 galaxies (e.g., Turner et al. 1997; Trippe et al. 2011; Marchese et al. 2014). In fact, this kind of variation has already been reported in the literature for objects included in the present work from intrinsic flux variations indicating changes in the nuclear continuum (Isobe et al. 2005) or because they needed to set free the normalization of the power law for a proper fit to the data (LaMassa et al. 2011). Also at higher energies, Soldi et al. (2014) studied the long-term variability of 110 AGN selected from the BAT 58-month survey and argue in favor of a variable nuclear continuum plus a constant reflection component. Their result is independent of the classification of the objects, which includes Seyferts, NLSy1s, radio galaxies, and quasars.

Flux variations are indeed a property of AGN, and they have been reported at different frequencies for Seyfert 2s, such as in radio (Nagar et al. 2002; Neff \& de Bruyn 1983) or infrared (Sharples et al. 1984; Hönig et al. 2012). In the present work we used data from the OM onboard XMM-Newton to study UV variability. These data are available at different epochs for six objects in our sample, but only NGC 5194 shows variations in the UVW1 filter. This is a Compton-thick candidate that does not vary in X-rays, so variations at UV frequencies from the nuclear component are not expected. It has been shown that the UV/optical spectra of Seyferts 2 include scattered AGN light, and it can sometimes be produced by young starbursts, including supernovae explosions (e.g., González Delgado et al. 2004). In fact, supernovae explosions in NGC 5194 have been reported in 1945, 1994, 2005, and 2011 (Van Dyk et al. 2011), which could account for the observed variations in the UV.

None of the remaining five nuclei show variations at UV frequencies, although there are two nuclei that are variable in X-rays (3C 98.0 and MARK 273). The lack of UV variations could be explained because X-ray and UV variations might not happen simultaneously (e.g., Hernández-García et al. 2014) or because we are not directly observing the nucleus. Muñoz Marín et al. (2009) studied 15 Seyfert galaxies with Hubble Space Telescope (HST) data (including types 1 and 2) and found that most type 2 nuclei appear resolved or absent at UV frequencies, concluding that the UV emission in Seyfert 2 s does not come from the nucleus. Thus, the lack of UV variations in Seyfert $2 \mathrm{~s}$ is most probably because we are not directly observing the nucleus at UV.

\subsection{Compton-thickness}

Brightman \& Nandra (2011a) show that at column densities $\sim 4 \times 10^{24} \mathrm{~cm}^{-2}$, the observed flux below $10 \mathrm{keV}$ is half that of the intrinsic flux at harder energies (see also Ghisellini et al. 1994). This indicates that in Compton-thick objects, the primary continuum is so absorbed in the $2-10 \mathrm{keV}$ energy band that the emission is optically thick to Compton scattering, and the spectrum is reflection-dominated. For this reason, we have 

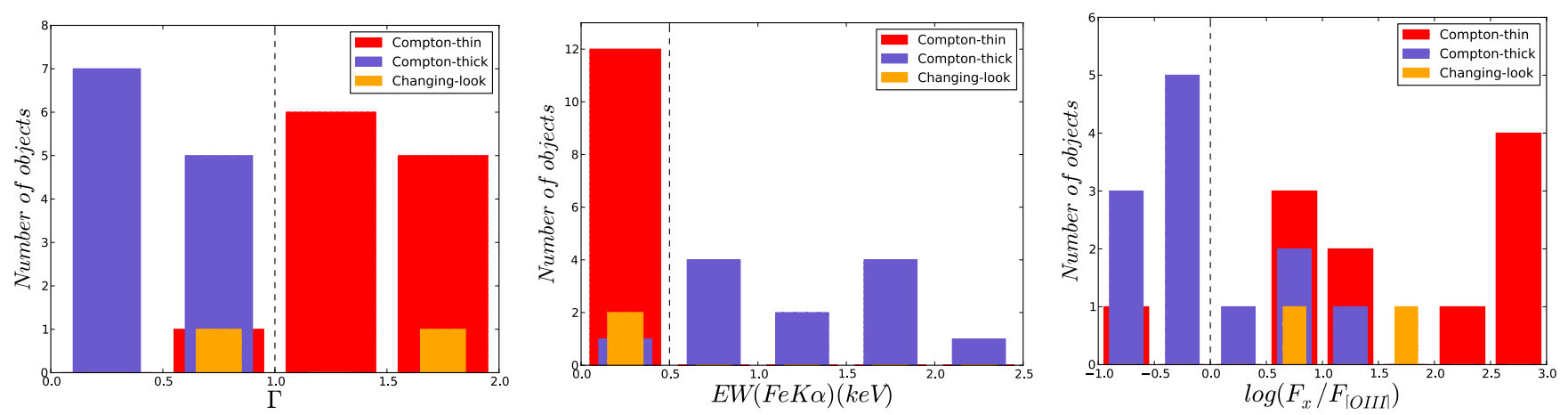

Fig. 5. Histograms of left: the slope of the power law, $\Gamma$ from Table A.7; middle: equivalent width of the iron line, $\mathrm{EW}(\mathrm{FeK} \alpha)$; and right: the X-ray to [O III] flux ratios. In all cases the sample is divided into Compton-thick (purple), Compton-thin (red), and changing-look (orange) candidates. The dashed lines represents the values for the selection of Compton-thick (below) and Compton-thin (above) candidates.

distinguished between Compton-thin and Compton-thick candidates (see Sects. 4.5 and 5.3).

However, the task of classifying Compton-thick objects with $\mathrm{X}$-ray data comprising energies up to $\sim 10 \mathrm{keV}$ is hard because the peak of the primary emission is above $10 \mathrm{keV}$. Instead, three different indicators involving X-ray and [O III] emission line data are used for their selection (see Sect. 4.5, for details). While the three criteria are met in most cases, our results have shown that the X-ray to [O III] line flux ratio, $\left.\log \left(F_{\mathrm{x}} / F_{[\mathrm{O}} \mathrm{III}\right]\right)$ is the most unsuitable indicator (see Fig. 5). This agrees with Brightman \& Nandra (2011b), who argue that this parameter can be inaccurate for classifying Compton-thick sources because of the uncertainty in the reddening correction of the [O III] line flux. Moreover, in Fig. 5 (right) there are four objects with $\log \left(F_{\mathrm{X}} / F_{[\mathrm{OIII}]}\right)>2.5$, which is higher than the values found by other authors (Bassani et al. 1999; Cappi et al. 2006; Panessa et al. 2006), what may be due to an underestimation of the [O III] line flux. Although the [O III] line is a good luminosity indicator, the reddening correction might depend on the geometry of the narrow line region, leading to an underestimation of its flux if we do not take it into account and leading to very high values of $F_{\mathrm{x}} / F_{\mathrm{OIII}}$.

In the present work, 12 nuclei are classified as Comptonthick candidates. Among them, variations are found only in MARK 3, which was previously classified as a Compton-thick candidate (Bassani et al. 1999; Goulding et al. 2012), with a column density of $1.1 \times 10^{24} \mathrm{~cm}^{-2}$ measured by BeppoSAX (Cappi et al. 1999). In fact, variations in MARK 3 have already been reported by Guainazzi et al. (2012), who studied its variability using XMM-Newton, Suzaku, and Swift data, and found variations on timescales of months. We found that the changes in MARK 3 are related to $\mathrm{Norm}_{2}$, i.e., intrinsic to the source. The most likely explanation for these variations could therefore be that part of the emission is still transmitted below $10 \mathrm{keV}$, so variations can be observed.

Interestingly, we found that most of the Compton-thick candidates are non-variable and tend to be fainter than Comptonthin and changing-look candidates, which show X-ray variations (see Fig. 4). This can be explained because the intrinsic luminosity is underestimated if the primary continuum is suppressed at energies below $10 \mathrm{keV}$, in agreement with the results of Brightman \& Nandra (2011a). In fact, the only Compton-thick candidate that shows variations in X-rays is included as a bright Seyfert 2 . It could be that variations are not observed because the spectra of Compton-thick sources are dominated by the reflection component. If so, this component might be located farther away from the central source, so it remains constant. This scenario agrees with the results we have obtained for the only source where a reflection component was statistically required by the data (namely $3 \mathrm{C}$ 98.0). These results are also in good agreement with those found by other authors, who did not find X-ray variability for objects classified as Compton-thick (e.g., NGC 424 and NGC 5194, LaMassa et al. 2011; Circinus, Arévalo et al. 2014; NGC 5643, Matt et al. 2013).

As noted above, if the reflection component does not vary, it might indicate that the reflection of the primary continuum occurs at large distances from the $\mathrm{SMBH}$. The same result was obtained by Risaliti (2002), who studied Seyfert 2s with BeppoSAX and found that the cold reflection component is compatible with being non-variable. They argue that if the reflection originates in the accretion disk, the reflection and the transmitted components must be closely related, but if the distance of the reflector to the $\mathrm{SMBH}$ is greater than the light crossing time of the intrinsic variations, the reflected component must remain constant. Therefore a reflector located far away from the SMBH is supported by our results, maybe in the torus or in the host galaxy.

\subsection{Caveats and limitations of the analysis}

The models used in this work to characterize the spectra of Seyfert 2 galaxies are a simplification of the true physical scenario occurring in these nuclei. In particular, the $2-10 \mathrm{keV}$ energy band - where variations are mostly found - is represented by an absorbed power law continuum, which could be an oversimplification of the real scenario.

Spectral variability analyses of seven sources studied in this work have been reported previously. Since at least some of these works study individual sources, the models used in their analyses might be more complex than ours (see Appendix B, for details). This comparison shows that our results are almost always compatible with those reported in the literature (MARK 1210, Matt et al. 2009 and Risaliti et al. 2010; NGC 4507, Matt et al. 2004; Marinucci et al. 2013; and Braito et al. 2013; MARK 273, Balestra et al. 2005; Circinus, Arévalo et al. 2014; NGC 6300, Guainazzi 2002; and NGC 7172, LaMassa et al. 2011). However, we cannot discard variations due to components that we did not fit in the models. For instance, Marchese et al. (2014) analyzed the XMM-Newton and Suzaku data of MARK 348 (also included in the present work), and report variations due to a neutral plus an ionized absorbers, together with a change in the ionization parameter of the ionized absorber. Their analysis is based on the residuals of the spectral fitting, where they include as many components as required, and the variability analysis is performed by testing different scenarios, including a variable continuum plus a constant reflection component 
$\left(\chi^{2} /\right.$ d.o.f. $\left.=567.7 / 407\right)$, a variable continuum plus a variable reflection component $\left(\chi^{2} /\right.$ d.o.f. $=551.1 / 406$, but variations are not observed), variations due to absorptions, and changes in the ionization state $\left(\chi^{2} /\right.$ d.o.f. $\left.=551.6 / 407\right)$. We notice that our spectral fit of MARK 348 with Norm $_{2}$ varies results in a very good fit $\left(\chi^{2} /\right.$ d.o.f. $\left.=1520.5 / 1368\right)$ when comparing the two $X M M-N e w t o n$ data sets, and residuals are mostly at energies below $\sim 2.5 \mathrm{keV}$ (see Fig. 2). Therefore, the presence of complex variations like these in at least some sources in our sample cannot be completely discarded.

\section{Conclusions}

Using Chandra and XMM-Newton public archives we performed a spectral, flux, short-, and long-term variability analysis of 26 optically selected Seyfert 2 galaxies. The main results of this study can be summarized as follows:

1. Long-term variability was found in 11 out of the 25 analyzed nuclei, which are more frequent among the brightest sources $\left(\log (L(2-10 \mathrm{keV}))>10^{42} \mathrm{erg} \mathrm{s}^{-1}\right)$. From the 11 variable sources, eight are Compton-thin candidates, two are changing-look, and only one (namely MARK 3 ) is a Compton-thick candidate. No difference in the variability is found among the HBLR and NHBLR objects. We report two changing-look candidates for the first time: MARK 273 and NGC 7319.

2. Short-term variability has not been detected in any of the sources. Nor UV variability.

3. The main driver of the observed variations is due to the power of the central engine manifested through variations in the normalization of the power law at high energies. At soft energies variations are rare, and column density variations have only been observed in four cases.

Our results are compatible with a scenario where a constant reflection component located far away from the nucleus and a variable nuclear continuum take place. Within this scenario, Compton-thick objects are dominated by reflection and do not show any X-ray spectral or flux variations. This implies that their luminosities are suppressed at hard X-rays, making them fainter sources than Compton-thin objects. In contrast, most of the Compton-thin or changing-look candidates are variable, showing different patterns of variability. These changes are mainly due to variations in the nuclear continuum. However, variations of the absorber or at soft energies are also found in some cases, with many of them accompanied by variations of the nuclear continuum. These variations are mainly due to clouds intersecting our line of sight.

Acknowledgements. We acknowledge the referee, M. Guainazzi, for his comments and suggestions that helped to improve the paper, and the AGN group at the IAA for helpful comments during this work. This work was financed by MINECO grant AYA 2010-15169, AYA 2013-42227-P, Junta de Andalucía TIC114 and Proyecto de Excelencia de la Junta de Andalucía P08-TIC-03531. L.H.G. acknowledges financial support from the Ministerio de Economía y Competitividad through the Spanish grant FPI BES-2011-043319. This research made use of data obtained from the Chandra Data Archive provided by the Chandra X-ray Center (CXC). This research made use of data obtained from the XMM-Newton Data Archive provided by the XMM-Newton Science Archive (XSA). This research made use of the NASA/IPAC extragalactic database (NED), which is operated by the Jet Propulsion Laboratory under contract with the National Aeronautics and Space Administration. We acknowledge the usage of the HyperLeda data base (http://leda.univ-lyon1. fr).

\section{References}

Afanas'ev, V. L., Lipovetskii, V. A., \& Shapovalova, A. I. 1981, Astrophys., 17, 342

Aguero, E. L., Calderon, J. H., Paolantonio, S., \& Suarez Boedo, E. 1994, PASP, 106, 978

Akylas, A., \& Georgantopoulos, I. 2009, A\&A, 500, 999

Akylas, A., Georgantopoulos, I., Griffiths, R. G., et al. 2002, MNRAS, 332, L23

Alloin, D., Bica, E., Bonatto, C., \& Prugniel, P. 1992, A\&A, 266, 117

Alonso-Herrero, A., Pereira-Santaella, M., Rieke, G. H., et al. 2013, ApJ, 765, 78

Antonucci, R. 1993, ARA\&A, 31, 473

Aoki, K., Kosugi, G., Wilson, A. S., \& Yoshida, M. 1999, ApJ, 521, 565 Arévalo, P., Bauer, F. E., Puccetti, S., et al. 2014, ApJ, 791, 81 Awaki, H., Koyama, K., Inoue, H., \& Halpern, J. P. 1991, PASJ, 43, 195 Awaki, H., Murakami, H., Leighly, K. M., et al. 2005, ApJ, 632, 793 Awaki, H., Murakami, H., Ogawa, Y., \& Leighly, K. M. 2006, ApJ, 645, 928 Balestra, I., Boller, T., Gallo, L., Lutz, D., \& Hess, S. 2005, A\&A, 442, 469 Baloković, M., Comastri, A., Harrison, F. A., et al. 2014, ApJ, 794, 111 Bassani, L., Dadina, M., Maiolino, R., et al. 1999, ApJS, 121, 473

Beckmann, V., Barthelmy, S. D., Courvoisier, T. J.-L., et al. 2007, A\&A, 475, 827

Bian, W., \& Gu, Q. 2007, ApJ, 657, 159

Bianchi, S., Guainazzi, M., Matt, G., et al. 2005a, A\&A, 442, 185

Bianchi, S., Miniutti, G., Fabian, A. C., \& Iwasawa, K. 2005b, MNRAS, 360, 380

Bianchi, S., Guainazzi, M., \& Chiaberge, M. 2006, A\&A, 448, 499

Bianchi, S., Piconcelli, E., Chiaberge, M., et al. 2009, ApJ, 695, 781

Bianchi, S., Chiaberge, M., Evans, D. A., et al. 2010, MNRAS, 405, 553

Bianchi, S., Panessa, F., Barcons, X., et al. 2012, MNRAS, 426, 3225

Boisson, C., Joly, M., Pelat, D., \& Ward, M. J. 2004, A\&A, 428, 373

Braito, V., Ballo, L., Reeves, J. N., et al. 2013, MNRAS, 428, 2516

Brightman, M., \& Nandra, K. 2011a, MNRAS, 413, 1206

Brightman, M., \& Nandra, K. 2011b, MNRAS, 414, 3084

Cappi, M., Bassani, L., Comastri, A., et al. 1999, A\&A, 344, 857

Cappi, M., Panessa, F., Bassani, L., et al. 2006, A\&A, 446, 459

Carilli, C. L., \& Taylor, G. B. 2000, ApJ, 532, L95

Collinge, M. J., \& Brandt, W. N. 2000, MNRAS, 317, L35

Comastri, A. 2004, in Supermassive Black Holes in the Distant Universe, ed. A. J. Barger, Astrophys. Space Sci. Libr., 308, 245

Corbett, E. A., Norris, R. P., Heisler, C. A., et al. 2002, ApJ, 564, 650

Costero, R., \& Osterbrock, D. E. 1977, ApJ, 211, 675

Davis, B. L., Berrier, J. C., Johns, L., et al. 2014, ApJ, 789, 124

de Rosa, A., Bassani, L., Ubertini, P., et al. 2008, A\&A, 483, 749

de Rosa, A., Panessa, F., Bassani, L., et al. 2012, MNRAS, 420, 2087

Dessauges-Zavadsky, M., Pindao, M., Maeder, A., \& Kunth, D. 2000, A\&A, 355,89

Dewangan, G. C., Griffiths, R. E., Choudhury, M., Miyaji, T., \& Schurch, N. J. 2005, ApJ, 635, 198

Diaz, A. I., Prieto, M. A., \& Wamsteker, W. 1988, A\&A, 195, 53

Dickey, J. M., \& Lockman, F. J. 1990, ARA\&A, 28, 215

Elmouttie, M., Haynes, R. F., Jones, K. L., Sadler, E. M., \& Ehle, M. 1998, MNRAS, 297, 1202

Eracleous, M., Hwang, J. A., \& Flohic, H. M. L. G. 2010, ApJ, 711, 796

Evans, D. A., Hardcastle, M. J., Croston, J. H., Worrall, D. M., \& Birkinshaw, M. 2005, MNRAS, 359, 363

Fairall, A. P. 1986, MNRAS, 218, 453

Falcke, H., Wilson, A. S., \& Simpson, C. 1998, ApJ, 502, 199

Ford, H. C., Crane, P. C., Jacoby, G. H., Lawrie, D. G., \& van der Hulst, J. M. 1985, ApJ, 293, 132

Fukazawa, Y., Iyomoto, N., Kubota, A., Matsumoto, Y., \& Makishima, K. 2001, A\&A, 374, 73

Garmire, G. P., Bautz, M. W., Ford, P. G., Nousek, J. A., \& Ricker, J. G. R. 2003, in SPIE Conf. Ser. 4851, eds. J. E. Truemper, \& H. D. Tananbaum, 28

Georgantopoulos, I., \& Zezas, A. 2003, ApJ, 594, 704

Ghisellini, G., Haardt, F., \& Matt, G. 1994, MNRAS, 267, 743

Gilli, R., Maiolino, R., Marconi, A., et al. 2000, A\&A, 355, 485

Gimeno, G. N., Díaz, R. J., \& Carranza, G. J. 2004, AJ, 128, 62

González Delgado, R. M., Cid Fernandes, R., Pérez, E., et al. 2004, ApJ, 605, 127

González-Martín, O., \& Vaughan, S. 2012, A\&A, 544, A80

González-Martín, O., Masegosa, J., Márquez, I., \& Guainazzi, M. 2009a, ApJ, 704,1570

González-Martín, O., Masegosa, J., Márquez, I., Guainazzi, M., \& Jiménez-Bailón, E. 2009b, A\&A, 506, 1107

González-Martín, O., Acosta-Pulido, J. A., Perez Garcia, A. M., \& Ramos Almeida, C. 2010, ApJ, 723, 1748

González-Martín, O., Papadakis, I., Braito, V., et al. 2011a, A\&A, 527, A142 
González-Martín, O., Papadakis, I., Reig, P., \& Zezas, A. 2011b, A\&A, 526, A132

González-Martín, O., Díaz-González, D., Acosta-Pulido, J. A., et al. 2014, A\&A, 567, A92

Goodrich, R. W., \& Osterbrock, D. E. 1983, ApJ, 269, 416

Goulding, A. D., Alexander, D. M., Bauer, F. E., et al. 2012, ApJ, 755, 5

Gu, Q., \& Huang, J. 2002, ApJ, 579, 205

Gu, Q., Melnick, J., Cid Fernandes, R., et al. 2006, MNRAS, 366, 480

Guainazzi, M. 2002, MNRAS, 329, L13

Guainazzi, M., Matt, G., Antonelli, L. A., et al. 1998, MNRAS, 298, 824

Guainazzi, M., Matt, G., Fiore, F., \& Perola, G. C. 2002, A\&A, 388, 787

Guainazzi, M., Fabian, A. C., Iwasawa, K., Matt, G., \& Fiore, F. 2005a, MNRAS, 356,295

Guainazzi, M., Matt, G., \& Perola, G. C. 2005b, A\&A, 444, 119

Guainazzi, M., La Parola, V., Miniutti, G., Segreto, A., \& Longinotti, A. L. 2012, A\&A, 547, A31

Hernández-García, L., González-Martín, O., Márquez, I., \& Masegosa, J. 2013, A\&A, 556, A47

Hernández-García, L., González-Martín, O., Masegosa, J., \& Márquez, I. 2014, A\&A, 569, A26

Ho, L. C., \& Ulvestad, J. S. 2001, ApJS, 133, 77

Ho, L. C., Filippenko, A. V., Sargent, W. L. W., \& Peng, C. Y. 1997, ApJS, 112, 391

Hönig, S. F., Kishimoto, M., Antonucci, R., et al. 2012, ApJ, 755, 149

Huchra, J. P., Wyatt, W. F., \& Davis, M. 1982, AJ, 87, 1628

Ishida, M., Tsujimoto, M., Kohmura, T., et al. 2011, PASJ, 63, 657

Isobe, N., Makishima, K., Tashiro, M., \& Hong, S. 2005, ApJ, 632, 781

Kalberla, P. M. W., Burton, W. B., Hartmann, D., et al. 2005, A\&A, 440, 775

Khachikian, E. Y., \& Weedman, D. W. 1974, ApJ, 192, 581

Khorunzhev, G. A., Sazonov, S. Y., Burenin, R. A., \& Tkachenko, A. Y. 2012 Astron. Lett., 38, 475

Kim, D.-C., Veilleux, S., \& Sanders, D. B. 1998, ApJ, 508, 627

Kinney, A. L., Antonucci, R. R. J., Ward, M. J., Wilson, A. S., \& Whittle, M. 1991, ApJ, 377, 100

Kirsch, M. G. F., Altieri, B., Chen, B., et al. 2004, in UV and Gamma-Ray Space Telescope Systems, eds. G. Hasinger, \& M. J. L. Turner, SPIE Conf. Ser., 5488,103

Komossa, S., \& Schulz, H. 1997, A\&A, 323, 31

Koski, A. T. 1978, ApJ, 223, 56

Kraemer, S. B., Schmitt, H. R., Crenshaw, D. M., et al. 2011, ApJ, 727, 130

Krolik, J. H., Madau, P., \& Zycki, P. T. 1994, ApJ, 420, L57

LaMassa, S. M., Heckman, T. M., Ptak, A., et al. 2011, ApJ, 729, 52

Leahy, J. P., Black, A. R. S., Dennett-Thorpe, J., et al. 1997, MNRAS, 291, 20

Levenson, N. A., Weaver, K. A., \& Heckman, T. M. 2001, ApJS, 133, 269

Liu, J.-F., \& Bregman, J. N. 2005, ApJS, 157, 59

Lumsden, S. L., Heisler, C. A., Bailey, J. A., Hough, J. H., \& Young, S. 2001, MNRAS, 327, 459

Magdziarz, P., \& Zdziarski, A. A. 1995, MNRAS, 273, 837

Maia, M. A. G., Machado, R. S., \& Willmer, C. N. A. 2003, AJ, 126, 1750

Maiolino, R., \& Rieke, G. H. 1995, ApJ, 454, 95

Maiolino, R., Salvati, M., Bassani, L., et al. 1998, A\&A, 338, 781

Marchese, E., Braito, V., Reeves, J. N., et al. 2014, MNRAS, 437, 2806

Marinucci, A., Bianchi, S., Matt, G., et al. 2011, A\&A, 526, A36

Marinucci, A., Risaliti, G., Wang, J., et al. 2013, MNRAS, 429, 2581

Marquez, I., \& Moles, M. 1996, A\&AS, 120, 1

Martini, P., Pogge, R. W., Ravindranath, S., \& An, J. H. 2001, ApJ, 562, 139

Matsumoto, C., Nava, A., Maddox, L. A., et al. 2004, ApJ, 617, 930

Matt, G., Guainazzi, M., Maiolino, R., et al. 1999, A\&A, 341, L39

Matt, G., Bianchi, S., Guainazzi, M., et al. 2003, A\&A, 399, 519

Matt, G., Bianchi, S., D’Ammando, F., \& Martocchia, A. 2004, A\&A, 421, 473

Matt, G., Bianchi, S., Awaki, H., et al. 2009, A\&A, 496, 653

Matt, G., Bianchi, S., Marinucci, A., et al. 2013, A\&A, 556, A91

Miller, J. S., \& Goodrich, R. W. 1990, ApJ, 355, 456

Moran, E. C., Barth, A. J., Kay, L. E., \& Filippenko, A. V. 2000, ApJ, 540, L73

Morganti, R., Tsvetanov, Z. I., Gallimore, J., \& Allen, M. G. 1999, A\&AS, 137, 457

Morris, S., Ward, M., Whittle, M., Wilson, A. S., \& Taylor, K. 1985, MNRAS, 216, 193

Muñoz Marín, V. M., Storchi-Bergmann, T., González Delgado, R. M., et al. 2009, MNRAS, 399, 842

Nagar, N. M., Wilson, A. S., Mulchaey, J. S., \& Gallimore, J. F. 1999, ApJS, 120,209
Nagar, N. M., Falcke, H., Wilson, A. S., \& Ulvestad, J. S. 2002, A\&A, 392, 53

Nandra, K., George, I. M., Mushotzky, R. F., Turner, T. J., \& Yaqoob, T. 1997, ApJ, 476, 70

Neff, S. G., \& de Bruyn, A. G. 1983, A\&A, 128, 318

Netzer, H., \& Turner, T. J. 1997, ApJ, 488, 694

Nicastro, F., Martocchia, A., \& Matt, G. 2003, ApJ, 589, L13

Noguchi, K., Terashima, Y., \& Awaki, H. 2009, ApJ, 705, 454

Oliva, E., Salvati, M., Moorwood, A. F. M., \& Marconi, A. 1994, A\&A, 288, 457

Oliva, E., Marconi, A., Cimatti, A., \& di Serego Alighieri, S. 1998, A\&A, 329, L21

Osterbrock, D. E., \& Martel, A. 1993, ApJ, 414, 552

Paggi, A., Wang, J., Fabbiano, G., Elvis, M., \& Karovska, M. 2012, ApJ, 756,

Panessa, F., \& Bassani, L. 2002, A\&A, 394, 435

Panessa, F., Bassani, L., Cappi, M., et al. 2006, A\&A, 455, 173

Pereira-Santaella, M., Alonso-Herrero, A., Santos-Lleo, M., et al. 2011, A\&A, 535, A93

Peterson, B. M. 1997, An Introduction to Active Galactic Nuclei (Cambridge University Press), 117, 314

Phillips, M. M., Charles, P. A., \& Baldwin, J. A. 1983, ApJ, 266, 485

Pogge, R. W., \& Eskridge, P. B. 1993, AJ, 106, 1405

Puccetti, S., Fiore, F., Risaliti, G., et al. 2007, MNRAS, 377, 607

Ramos Almeida, C., Pérez García, A. M., Acosta-Pulido, J. A., \& González-Martín, O. 2008, ApJ, 680, L17

Rees, M. J. 1984, ARA\&A, 22, 471

Risaliti, G. 2002, A\&A, 386, 379

Risaliti, G., Elvis, M., \& Nicastro, F. 2002, ApJ, 571, 234

Risaliti, G., Elvis, M., Fabbiano, G., et al. 2007, ApJ, 659, L111

Risaliti, G., Elvis, M., Bianchi, S., \& Matt, G. 2010, MNRAS, 406, L20

Risaliti, G., Nardini, E., Salvati, M., et al. 2011, MNRAS, 410, 1027

Rodríguez-Baras, M., Rosales-Ortega, F. F., Díaz, A. I., Sánchez, S. F., \& Pasquali, A. 2014, MNRAS, 442, 495

Ryder, S. D., Buta, R. J., Toledo, H., et al. 1996, ApJ, 460, 665

Severgnini, P., Caccianiga, A., \& Della Ceca, R. 2012, A\&A, 542, A46

Sharples, R. M., Longmore, A. J., Hawarden, T. G., \& Carter, D. 1984, MNRAS, 208, 15

Singh, V., Shastri, P., \& Risaliti, G. 2011, A\&A, 532, A84

Smith, M. G. 1975, ApJ, 202, 591

Smith, D. A., Georgantopoulos, I., \& Warwick, R. S. 2001, ApJ, 550, 635

Sobolewska, M. A., \& Papadakis, I. E. 2009, MNRAS, 399, 1597

Soldi, S., Beckmann, V., Baumgartner, W. H., et al. 2014, A\&A, 563, A57

Strüder, L., Briel, U., Dennerl, K., et al. 2001, A\&A, 365, L18

Teng, S. H., Veilleux, S., Anabuki, N., et al. 2009, ApJ, 691, 261

Terashima, Y., \& Wilson, A. S. 2001, ApJ, 560, 139

Tran, H. D. 1995, ApJ, 440, 578

Tran, H. D., Miller, J. S., \& Kay, L. E. 1992, ApJ, 397, 452

Tremaine, S., Gebhardt, K., Bender, R., et al. 2002, ApJ, 574, 740

Trinchieri, G., Sulentic, J., Breitschwerdt, D., \& Pietsch, W. 2003, A\&A, 401, 173

Trippe, M. L., Reynolds, C. S., Koss, M., Mushotzky, R. F., \& Winter, L. M. 2011, ApJ, 736, 81

Trotter, A. S., Greenhill, L. J., Moran, J. M., et al. 1998, ApJ, 495, 740

Tsujimoto, M., Guainazzi, M., Plucinsky, P. P., et al. 2011, A\&A, 525, A25

Turner, T. J., George, I. M., Nandra, K., \& Mushotzky, R. F. 1997, ApJS, 113, 23

Ulvestad, J. S., \& Wilson, A. S. 1984, ApJ, 278, 544

Ulvestad, J. S., \& Wilson, A. S. 1989, ApJ, 343, 659

Unger, S. W., Lawrence, A., Wilson, A. S., Elvis, M., \& Wright, A. E. 1987, MNRAS, 228, 521

Urry, C. M., \& Padovani, P. 1995, PASP, 107, 803

Van Dyk, S. D., Li, W., Cenko, S. B., et al. 2011, ApJ, 741, L28

Vaughan, S., Edelson, R., Warwick, R. S., \& Uttley, P. 2003, MNRAS, 345, 1271

Veilleux, S., \& Osterbrock, D. E. 1987, ApJS, 63, 295

Veilleux, S., Kim, D.-C., Sanders, D. B., Mazzarella, J. M., \& Soifer, B. T. 1995 ApJS, 98, 171

Veron, P., Goncalves, A. C., \& Veron-Cetty, M.-P. 1997, A\&A, 319, 52

Véron-Cetty, M.-P., \& Véron, P. 2010, A\&A, 518, A10

Xanthopoulos, E., Muxlow, T. W. B., Thomasson, P., \& Garrington, S. T. 2004, MNRAS, 353, 1117

Zaw, I., Farrar, G. R., \& Greene, J. E. 2009, ApJ, 696, 1218

Pages 19 to 77 are available in the electronic edition of the journal at http://www . aanda. org 


\section{Appendix A: Tables}

Table A.1. Observational details.

\begin{tabular}{|c|c|c|c|c|c|c|c|c|}
\hline Name & Instrument & ObsID & Date & $\begin{array}{l}R \\
(")\end{array}$ & $\begin{array}{c}\text { Net Exptime } \\
(\mathrm{ks})\end{array}$ & Counts & $\begin{array}{c}\log \left(L_{\mathrm{UV}}\right) \\
(\mathrm{erg} / \mathrm{s})\end{array}$ & Filter \\
\hline (1) & (2) & (3) & (4) & (5) & (6) & (7) & (8) & (9) \\
\hline \multirow[t]{2}{*}{ MARK 348} & XMM-Newton & 0067540201 & $2002-07-18$ & 25 & 18.5 & 39552 & - & \\
\hline & XMM-Newton & 0701180101 & 2013-01-04 & 25 & 7.2 & 5681 & - & \\
\hline \multirow[t]{3}{*}{ NGC 424} & XMM-Newton & $0002942301^{c}$ & 2001-12-10 & 20 & 4.5 & 1777 & - & \\
\hline & XMM-Newton & 0550950101 & 2008-12-07 & 20 & 127.5 & 33452 & - & \\
\hline & Chandra & $3146^{c}$ & 2002-02-04 & 2 & 9.2 & 1266 & - & \\
\hline \multirow[t]{4}{*}{ MARK 573} & Chandra & $7745^{c}$ & 2006-11-18 & 2 & 38.1 & 3181 & - & \\
\hline & Chandra & 13124 & 2010-09-17 & 2 & 52.4 & 3456 & - & \\
\hline & XMM-Newton & $0200430701^{c}$ & 2004-01-15 & 20 & 9.0 & 3605 & $42.75_{-}^{+} 0.01$ & UVW1 \\
\hline & & & & & & & $42.50_{-}^{+} 0.05$ & UVW2 \\
\hline \multirow[t]{2}{*}{ NGC 788} & XMM-Newton & $0601740201^{c}$ & $2010-01-15$ & 20 & 12.0 & 4464 & - & \\
\hline & Chandra & $11680^{c}$ & 2009-09-06 & 3 & 13.6 & 1155 & - & \\
\hline \multirow[t]{2}{*}{ ESO 417-G06 } & XMM-Newton & 0602560201 & 2009-07-11 & 20 & 5.9 & 2273 & - & \\
\hline & XMM-Newton & 0602560301 & 2009-08-20 & 20 & 6.1 & 2031 & - & \\
\hline \multirow[t]{2}{*}{ MARK 1066} & Chandra & $4075^{c}$ & $2003-07-14$ & 3 & 19.9 & 807 & - & \\
\hline & XMM-Newton & 0201770201 & $2005-02-20$ & 20 & 7.6 & 974 & - & \\
\hline \multirow[t]{3}{*}{ 3С 98.0} & XMM-Newton & 0064600101 & 2002-09-07 & 20 & 9.5 & 2453 & $41.94_{-}^{+} 0.08$ & UVW1 \\
\hline & XMM-Newton & $0064600301^{c}$ & 2003-02-05 & 20 & 2.9 & 422 & $41.99_{-}^{+} 0.07$ & UVW1 \\
\hline & Chandra & $10234^{c}$ & $2008-12-24$ & 2 & 31.7 & 1353 & - & \\
\hline \multirow[t]{7}{*}{ MARK 3} & XMM-Newton & 0111220201 & $2000-10-19$ & 30 & 35.2 & 30700 & - & \\
\hline & XMM-Newton & 0009220601 & 2001-03-20 & 30 & 4.3 & 3471 & - & \\
\hline & XMM-Newton & 0009220701 & 2001-03-28 & 30 & 3.1 & 2465 & - & \\
\hline & XMM-Newton & 0009220901 & $2001-09-12$ & 30 & 0.9 & 708 & - & \\
\hline & $X M M-N e w t o n$ & 0009220401 & $2002-03-10$ & 30 & 2.7 & 2215 & - & \\
\hline & XMM-Newton & 0009220501 & $2002-03-25$ & 30 & 4.3 & 3512 & - & \\
\hline & XMM-Newton & 0009221601 & 2002-09-16 & 30 & 1.3 & 1042 & - & \\
\hline \multirow[t]{5}{*}{ MARK 1210} & Chandra & 4875 & 2004-03-04 & 2 & 10.4 & 1998 & - & \\
\hline & Chandra & 9264 & $2008-02-15$ & 2 & 9.8 & 2052 & - & \\
\hline & Chandra & 9265 & $2008-02-15$ & 2 & 9.4 & 1873 & - & \\
\hline & Chandra & 9266 & $2008-02-15$ & 2 & 9.4 & 1752 & - & \\
\hline & Chandra & 9268 & 2008-03-06 & 2 & 9.8 & 1608 & - & \\
\hline \multirow[t]{2}{*}{ NGC 3079} & Chandra & $2038^{c}$ & 2001-03-07 & 4 & 27 & 414 & - & \\
\hline & XMM-Newton & $0110930201^{c}$ & 2001-04-13 & 25 & 5 & 1112 & - & \\
\hline \multirow[t]{2}{*}{ IC 2560} & XMM-Newton & $0203890101^{c}$ & 2003-12-26 & 20 & 70.7 & 7694 & - & \\
\hline & Chandra & $4908^{c}$ & $2004-02-16$ & 3 & 55.4 & 1583 & - & \\
\hline \multirow[t]{3}{*}{ NGC 3393} & Chandra & $4868^{c}$ & $2004-02-28$ & 5 & 29.3 & 1971 & - & \\
\hline & Chandra & 12290 & 2011-03-12 & 5 & 69.2 & 3716 & - & \\
\hline & XMM-Newton & $0140950601^{c}$ & 2003-07-05 & 20 & 10.1 & 2759 & - & \\
\hline
\end{tabular}

Notes. Column 1: name; Col. 2: instrument; Col. 3: obsID; Col. 4: date; Col. 5: aperture radius for the nuclear extraction; Col. 6: net exposure time; Col. 7: number of counts in the $0.5-10 \mathrm{keV}$ band; Cols. 8 and 9: UV luminosity from the optical monitor and filter. The $c$ represents data from different instruments that were compared as explained in Sect. 4.2. 
Table A.1. continued.

\begin{tabular}{|c|c|c|c|c|c|c|c|c|}
\hline Name & Instrument & ObsID & Date & $\begin{array}{l}R \\
\left({ }^{\prime \prime}\right) \\
(5)\end{array}$ & $\begin{array}{l}\text { Net Exptime } \\
\text { (ks) } \\
(6)\end{array}$ & Counts & $\begin{array}{c}\log \left(L_{\mathrm{UV}}\right) \\
(\mathrm{erg} / \mathrm{s}) \\
(8)\end{array}$ & Filter \\
\hline \multirow[t]{7}{*}{ NGC 4507} & XMM-Newton & 0006220201 & 2001-01-04 & 30 & 32.3 & 35004 & - & \\
\hline & $X M M-N e w t o n$ & 0653870201 & 2010-06-24 & 30 & 15.1 & 11977 & - & \\
\hline & XMM-Newton & 0653870301 & 2010-07-03 & 30 & 12.1 & 9574 & - & \\
\hline & XMM-Newton & $0653870401^{c}$ & $2010-07-13$ & 30 & 12.2 & 10023 & - & \\
\hline & XMM-Newton & 0653870501 & $2010-07-23$ & 30 & 10.3 & 8247 & - & \\
\hline & XMM-Newton & 0653870601 & 2010-08-03 & 30 & 1.0 & 752 & - & \\
\hline & Chandra & $12292^{c}$ & 2010-12-02 & 2 & 39.6 & 9048 & & \\
\hline \multirow[t]{2}{*}{ NGC 4698} & XMM-Newton & 0112551101 & $2001-12-16$ & 25 & 8 & 411 & $40.14_{-}^{+} 0.10$ & UVM2 \\
\hline & XMM-Newton & 0651360401 & 2010-06-09 & 25 & 28 & 1647 & $40.14_{-}^{+} 0.11$ & UVM2 \\
\hline \multirow[t]{20}{*}{ NGC 5194} & Chandra & 1622 & 2001-06-23 & 2 & 27 & 451 & - & \\
\hline & Chandra & $3932^{c}$ & 2003-08-07 & 2 & 48 & 940 & - & \\
\hline & Chandra & 13813 & 2012-09-09 & 2 & 179.2 & 2238 & - & \\
\hline & Chandra & 13812 & 2012-09-12 & 2 & 157.5 & 2516 & - & \\
\hline & Chandra & 13814 & 2012-09-20 & 2 & 189.9 & 2574 & - & \\
\hline & Chandra & 13815 & $2012-09-23$ & 2 & 67.2 & 1022 & - & \\
\hline & Chandra & 13816 & 2012-09-26 & 2 & 73.1 & 1033 & - & \\
\hline & $X M M-N e w t o n$ & $0112840201^{c}$ & 2003-01-15 & 25 & 17 & 11641 & $40.94_{-}^{+} 0.01$ & UVW1 \\
\hline & $X M M-N e w t o n$ & 0212480801 & 2005-07-01 & - & - & - & $40.93_{-}^{+} 0.01$ & UVW1 \\
\hline & & & & & & & $40.38_{-}^{+} 0.11$ & UVM2 \\
\hline & & & & & & & $40.37_{-}^{+} 0.16$ & UVW2 \\
\hline & $X M M-N e w t o n$ & 0303420101 & $2006-05-20$ & - & - & - & $40.79_{-}^{+} 0.01$ & UVW1 \\
\hline & XMM-Newton & 0303420201 & $2006-05-24$ & - & - & - & $40.84_{-}^{+} 0.01$ & UVW1 \\
\hline & & & & & & & $40.34_{-}^{+} 0.07$ & UVW2 \\
\hline & $X M M-N e w t o n$ & 0677980701 & 2011-06-07 & - & - & - & $40.97_{-}^{+} 0.01$ & UVW1 \\
\hline & & & & & & & $40.59_{-}^{+} 0.04$ & UVM2 \\
\hline & & & & & & & $40.40_{-}^{+} 0.08$ & UVW2 \\
\hline & $X M M-N e w t o n$ & 0677980801 & 2011-06-11 & - & - & - & $40.94_{-}^{+} 0.01$ & UVW1 \\
\hline & & & & & & & $40.53_{-}^{+} 0.04$ & UVM2 \\
\hline & & & & & & & $40.41_{-}^{+} 0.08$ & UVW2 \\
\hline \multirow[t]{4}{*}{ MARK 268} & XMM-Newton & 0554500701 & $2008-07-20$ & 20 & 2.3 & 547 & $42.59_{-}^{+} 0.05$ & UVM2 \\
\hline & & & & & & & $42.93_{-}^{+} 0.01$ & UVW1 \\
\hline & XMM-Newton & 0554501101 & 2008-07-22 & 20 & 10.5 & 2469 & $42.66_{-}^{+} 0.064$ & UVM2 \\
\hline & & & & & & & $42.92_{-}^{+} 0.01$ & UVW1 \\
\hline \multirow[t]{3}{*}{ MARK 273} & XMM-Newton & $0101640401^{c}$ & $2002-05-07$ & 20 & 17.8 & 1796 & $43.05_{-}^{+} 0.06$ & UVW1 \\
\hline & XMM-Newton & 0651360301 & 2010-05-13 & - & - & - & $43.16_{-}^{+} 0.01$ & UVW1 \\
\hline & Chandra & $809^{c}$ & 2000-04-19 & 4 & 44.2 & 1633 & - & \\
\hline \multirow[t]{5}{*}{ Circinus } & Chandra & 365 & $2000-03-14$ & 2 & 5.0 & 1638 & - & \\
\hline & Chandra & 9140 & $2008-10-26$ & 2 & 48.8 & 15594 & - & \\
\hline & Chandra & $10937^{c}$ & $2009-12-28$ & 2 & 18.3 & 5929 & - & \\
\hline & $X M M-N e w t o n$ & 0111240101 & 2001-08-06 & 15 & 63.8 & 139614 & - & \\
\hline & XMM-Newton & $0656580601^{c}$ & 2014-03-01 & 15 & 24.1 & 43031 & - & \\
\hline \multirow[t]{2}{*}{ NGC 5643} & XMM-Newton & 0140950101 & 2003-02-08 & 25 & 5.9 & 1419 & - & \\
\hline & XMM-Newton & 0601420101 & 2009-07-25 & 25 & 16.1 & 4142 & - & \\
\hline \multirow[t]{2}{*}{ MARK 477} & XMM-Newton & 0651100301 & $2010-07-21$ & 20 & 7.2 & 1898 & $43.41_{-}^{+} 0.01$ & UVW1 \\
\hline & XMM-Newton & 0651100401 & 2010-07-23 & 20 & 6.5 & 1761 & $43.43_{-}^{+} 0.01$ & UVW1 \\
\hline
\end{tabular}


L. Hernández-García et al.: X-ray variability of Seyfert 2s

Table A.1. continued.

\begin{tabular}{|c|c|c|c|c|c|c|c|c|}
\hline Name & Instrument & ObsID & Date & $\begin{array}{c}R \\
\left({ }^{\prime \prime}\right)\end{array}$ & $\begin{array}{c}\text { Net Exptime } \\
(\mathrm{ks})\end{array}$ & Counts & $\begin{array}{c}\log \left(L_{\mathrm{UV}}\right) \\
(\mathrm{erg} / \mathrm{s})\end{array}$ & Filter \\
\hline (1) & (2) & (3) & (4) & (5) & (6) & (7) & (8) & (9) \\
\hline \multirow[t]{2}{*}{ IC 4518A } & XMM-Newton & 0401790901 & 2006-08-07 & 20 & 7.5 & 2082 & - & \\
\hline & XMM-Newton & 0406410101 & 2006-08-15 & 20 & 21.1 & 4003 & - & \\
\hline \multirow[t]{2}{*}{ ESO 138-G01 } & XMM-Newton & 0405380201 & $2007-02-16$ & 20 & 10.5 & 4454 & - & \\
\hline & XMM-Newton & 0690580101 & $2013-02-24$ & 20 & 7.7 & 3179 & - & \\
\hline \multirow[t]{3}{*}{ NGC 6300} & Chandra & $10292^{c}$ & 2009-06-10 & 2 & 9.8 & 3686 & - & \\
\hline & Chandra & 10293 & 2009-06-14 & 2 & 9.8 & 3331 & - & \\
\hline & XMM-Newton & $0059770101^{c}$ & 2001-03-02 & 20 & 34.9 & 919 & - & \\
\hline \multirow[t]{3}{*}{ NGC 7172} & XMM-Newton & 0147920601 & 2002-11-18 & 25 & 10.9 & 19949 & - & \\
\hline & XMM-Newton & 0202860101 & 2004-11-11 & 25 & 18.1 & 31517 & - & \\
\hline & XMM-Newton & 0414580101 & 2007-04-24 & 25 & 26.9 & 92998 & - & \\
\hline \multirow[t]{2}{*}{ NGC 7212} & XMM-Newton & $0200430201^{c}$ & $2004-05-20$ & 20 & 9.6 & 1365 & Not detected & \\
\hline & Chandra & $4078^{c}$ & 2003-07-22 & 3 & 19.9 & 682 & - & \\
\hline \multirow[t]{3}{*}{ NGC 7319} & Chandra & 789 & 2000-07-09 & 3 & 19.7 & 880 & - & \\
\hline & Chandra & $7924^{c}$ & $2007-08-17$ & 3 & 93.2 & 3796 & - & \\
\hline & XMM-Newton & $0021140201^{c}$ & 2001-12-07 & 20 & 32.3 & 5839 & Not detected & \\
\hline
\end{tabular}


Table A.2. Final compilation of the best-fit models for the sample, including the individual best-fit model for each observation, and the simultaneous best-fit model with the varying parameters.

\begin{tabular}{|c|c|c|c|c|c|c|c|c|c|}
\hline Analysis & ObsID & Model & $N_{\mathrm{H} 1}$ & $N_{\mathrm{H} 2}$ & $\begin{array}{l}k T \\
\mathrm{keV} \\
(6)\end{array}$ & (7) & $\begin{array}{c}\text { Norm }_{1} \\
\left(10^{-4}\right) \\
(8)\end{array}$ & $\begin{array}{c}\mathrm{Norm}_{2} \\
\left(10^{-4}\right) \\
(9)\end{array}$ & $\begin{array}{c}\chi^{2} / \text { d.o.f. } \\
\text { F-test } \\
(10)\end{array}$ \\
\hline \multicolumn{10}{|c|}{ MARK 348} \\
\hline Ind & $0067540201 *$ & ME2PL & $0.00_{0.00}^{0.03}$ & $13.40_{12.94}^{13.61}$ & $0.18_{0.14}^{0.20}$ & $1.50_{1.44}^{1.56}$ & $0.59_{0.55}^{0.63}$ & $80.76_{80.76}^{101.65}$ & $1281.96 / 1132$ \\
\hline Ind & 0701180101 & ME2PL & - & $12.89_{11.74}^{14.12}$ & $0.20_{0.18}^{0.23}$ & $1.42_{1.26}^{1.58}$ & $0.37_{0.37}^{0.49}$ & $23.81_{17.39}^{32.79}$ & $219.05 / 227$ \\
\hline \multirow[t]{3}{*}{ SMF1 } & 0067540201 & ME2PL & - & $13.29_{12.90}^{13.70}$ & $0.19_{0.18}^{0.21}$ & $1.50_{1.44}^{1.56}$ & $0.54_{0.50}^{0.57}$ & $88.49_{79.91}^{98.95}$ & $1520.54 / 1368$ \\
\hline & 0701180101 & & & & & & & $27.17_{24.37}^{30.49}$ & 0 \\
\hline & \multicolumn{9}{|c|}{ NGC 424} \\
\hline Ind & 0002942301* & 2ME2PL & - & $34.89_{23.92}^{51.68}$ & $0.07_{0.06}^{0.09}\left(0.65_{0.58}^{0.72}\right)$ & $1.49_{1.07}^{1.91}$ & $0.48_{0.48}^{0.85}$ & $4.21_{1.44}^{10.69}$ & $66.80 / 54$ \\
\hline Ind & 0550950101 & 2ME2PL & - & $45.55_{41.29}^{51.01}$ & $0.10_{0.09}^{0.11}\left(0.71_{0.70}^{0.72}\right)$ & $2.03_{1.93}^{2.10}$ & $0.74_{0.74}^{0.83}$ & $11.47_{9.26}^{13.75}$ & $1165.90 / 532$ \\
\hline Ind & 3146 & 2ME2PL & - & $17.12_{13.14}^{22.84}$ & $0.10_{0.08}^{0.15}\left(0.71_{0.60}^{0.84}\right)$ & $2.35_{1.94}^{2.63}$ & $0.68_{0.68}^{1.01}$ & $13.76_{6.62}^{23.77}$ & $48.02 / 37$ \\
\hline SMF0 & $0002942301 / 3146$ & 2ME2PL & - & $24.49_{18.65}^{31.94}$ & $0.09_{0.07}^{0.10}\left(0.67_{0.61}^{0.73}\right)$ & $1.82_{1.46}^{2.15 \dagger}$ & $0.72_{0.58}^{0.88}$ & $6.70_{3.11}^{13.11}$ & $138.97 / 103$ \\
\hline \multicolumn{10}{|c|}{ MARK 573} \\
\hline Ind & 7745 & 2ME2PL & - & $33.28_{15.65}^{98.24}$ & $0.13_{0.11}^{0.15}\left(0.71_{0.66}^{0.76}\right)$ & $2.50_{2.02}^{2.78}$ & $0.48_{0.36}^{0.55}$ & $3.97_{1.51}^{16.71}$ & $71.22 / 67$ \\
\hline Ind & $13124 *$ & 2ME2PL & - & $38.48_{29.40}^{68.89}$ & $0.09_{0.07}^{0.13}\left(0.67_{0.62}^{0.72}\right)$ & $1.92_{1.61}^{2.27}$ & $0.57_{0.57}^{0.87}$ & $5.18_{2.49}^{11.29}$ & $92.51 / 78$ \\
\hline Ind & 0200430701 & 2ME2PL & - & $17.12_{10.02}^{28.98}$ & $0.14_{0.11}^{0.18}\left(0.73_{0.68}^{0.82}\right)$ & $3.23_{3.03}^{3.45}$ & $0.66_{0.66}^{0.87}$ & $9.85_{5.19}^{18.62}$ & $78.04 / 88$ \\
\hline SMF0 & All & 2ME2PL & - & $45.83_{25.56}^{103.01}$ & $0.10_{0.09}^{0.12}\left(0.67_{0.64}^{0.69}\right)$ & $2.12_{1.85}^{2.45 \dagger}$ & $0.41_{0.38}^{0.51}$ & $2.74 \begin{array}{l}5.16 \\
1.37\end{array}$ & $198.73 / 161$ \\
\hline \multicolumn{10}{|c|}{ NGC 788} \\
\hline Ind & $0601740201 *$ & 2ME2PL & - & $50.32_{44.62}^{56.40}$ & $0.11_{0.09}^{0.12}\left(0.71_{0.64}^{0.76}\right)$ & $1.41_{1.15}^{1.67}$ & $0.31_{0.31}^{0.47}$ & $16.84_{9.18}^{30.52}$ & $199.77 / 154$ \\
\hline Ind & 11680 & 2ME2PL & - & $44.35_{36.55}^{53.07}$ & $0.14_{0.09}^{0.17}\left(0.76_{0.67}^{0.87}\right)$ & $0.61_{0.15}^{1.06}$ & $0.15_{0.15}^{0.31}$ & $4.10_{1.53}^{10.51}$ & $34.4566 / 39$ \\
\hline SMF0 & All(+ring) & 2ME2PL & - & $46.61 \begin{array}{l}51.35 \\
42.14\end{array}$ & $0.11_{0.09}^{0.12}\left(0.71_{0.67}^{0.75}\right)$ & $1.288_{1.06}^{1.51}$ & $0.35_{0.29}^{0.41}$ & $12.43_{7.55}^{20.51}$ & $262.36 / 205$ \\
\hline \multicolumn{10}{|c|}{ ESO 417-G06 } \\
\hline Ind & $0602560201^{*}$ & MEPL & $0.77_{0.57}^{0.91}$ & $5.15_{4.41}^{6.10}$ & $0.13_{0.10}^{0.18}$ & $1.03_{0.85}^{1.25}$ & $59.98_{10.32}^{571.31}$ & $4.52_{3.22}^{6.40}$ & $129.13 / 96$ \\
\hline Ind & 0602560301 & MEPL & $0.72_{0.50}^{0.87}$ & $7.85_{6.70}^{9.19}$ & $0.19_{0.14}^{0.26}$ & $1.44_{1.20}^{1.70}$ & $16.28_{2.52}^{102.57}$ & $8.56_{5.48}^{13.80}$ & $108.13 / 85$ \\
\hline SMF1 & 0602560201 & MEPL & $0.76_{0.62}^{0.84}$ & $5.64 \begin{array}{l}6.38 \\
5.01\end{array}$ & $0.15_{0.14}^{0.19}$ & $1.21_{1.07}^{1.37}$ & $46.28_{9.33}^{110.75}$ & $5.91_{4.57}^{7.95}$ & $249.86 / 189$ \\
\hline & 0602560301 & & & $7.16_{6.42}^{8.02}$ & & & & & $2.6 e-5$ \\
\hline \multicolumn{10}{|c|}{ MARK 1066} \\
\hline Ind & 4075 & ME2PL & $0.25_{0.13}^{0.47}$ & $70.47_{18.90}^{186.87}$ & $0.65_{0.59}^{0.71}$ & $2.17_{1.85}^{2.40}$ & $0.40_{0.40}^{1.20}$ & $5.66_{0.22}^{20.92}$ & $41.68 / 24$ \\
\hline Ind & $0201770201 *$ & ME2PL & $0.09_{0.00}^{0.19}$ & $54.30_{30.42}^{106.19}$ & $0.76_{0.61}^{0.86}$ & $2.17_{1.57}^{2.68}$ & $0.45_{0.45}^{1.05}$ & $6.23_{1.74}^{19.76}$ & $27.86 / 35$ \\
\hline SMF0 & All & ME2PL & $0.12_{0.03}^{0.24}$ & $82.29_{45.83}^{175.04}$ & $0.68_{0.62}^{0.79}$ & $2.02_{1.71}^{2.36 \dagger}$ & $0.53_{0.40}^{0.71}$ & $7.11_{2.23}^{25.58}$ & $96.11 / 69$ \\
\hline \multicolumn{10}{|c|}{$3 \mathrm{C} 98.0$} \\
\hline Ind & $0064600101 *$ & MEPL & $0.67_{0.57}^{0.76}$ & $7.08_{6.03}^{8.28}$ & $0.15_{0.12}^{0.17}$ & $1.04_{0.81}^{1.27}$ & $91.66_{37.02}^{337.94}$ & $3.27_{2.21}^{4.95}$ & $117.19 / 102$ \\
\hline Ind & 0064600301 & MEPL & $0.69_{0.52}^{1.00}$ & $7.15_{4.61}^{10.52}$ & $0.17_{0.11}^{0.21}$ & $0.99_{0.48}^{1.64}$ & $40.52_{9.40}^{138.52}$ & $1.75_{0.70}^{5.60}$ & $7.30 / 12$ \\
\hline Ind & 10234 & MEPL & $1.34_{0.00}^{2.02}$ & $7.07_{5.56}^{9.09}$ & $0.21_{0.10}^{0.25}$ & $1.04_{0.66}^{1.48}$ & $13.04_{0.25}^{662.07}$ & $1.73_{0.90}^{4.18}$ & $64.69 / 53$ \\
\hline \multirow[t]{3}{*}{ SMF1 } & 0064600101 & MEPL & $0.69_{0.61}^{0.77}$ & $7.08_{6.11}^{8.20}$ & $0.14_{0.13}^{0.17}$ & $1.02_{0.83}^{1.23}$ & $92.50_{36.51}^{231.65}$ & $3.22_{2.24}^{4.75}$ & $127.89 / 122$ \\
\hline & 0064600301 & & & & & & & $1.85_{1.27}^{2.74}$ & 6.1e-19 \\
\hline & \multicolumn{9}{|c|}{ MARK 3} \\
\hline Ind & $0111220201^{*}$ & 2ME2PL & - & $44.07_{41.14}^{47.24}$ & $0.16_{0.14}^{0.17}\left(0.69_{0.67}^{0.71}\right)$ & $1.25_{1.17}^{1.33}$ & $1.54_{1.54}^{1.74}$ & $15.89_{13.17}^{19.10}$ & $934.21 / 789$ \\
\hline Ind & 0009220601 & 2ME2PL & - & $43.05_{34.88}^{53.76}$ & $0.12_{0.09}^{0.18}\left(0.67_{0.63}^{0.73}\right)$ & $1.37_{1.12}^{1.62}$ & $1.38_{1.38}^{2.01}$ & $14.55_{8.03}^{25.44}$ & $162.96 / 134$ \\
\hline Ind & 0009220701 & 2ME2PL & - & $38.53_{28.53}^{53.89}$ & $0.11_{0.06}^{0.18}\left(0.79_{0.73}^{0.86}\right)$ & $1.54_{1.21}^{1.84}$ & $1.43_{1.42}^{2.30}$ & $18.14_{8.68}^{34.58}$ & $93.31 / 93$ \\
\hline Ind & 0009220901 & 2ME2PL & - & $24.25_{10.54}^{92.09}$ & $0.12_{0.07}^{0.19}\left(0.59_{0.46}^{0.69}\right)$ & $0.97_{0.31}^{1.61}$ & $0.78_{0.78}^{2.03}$ & $3.48_{0.31}^{14.84}$ & $10.50 / 21$ \\
\hline
\end{tabular}

Notes. Column 1: kind of analysis performed, where Ind refers to the individual fitting of the observation, SMF0 is the simultaneous fit without varying parametes, SMF1 the simultaneous fit varying one parameter, and SMF2 the simultaneous fit varying two parameters; Col. 2: obsID, where the $\left(^{*}\right)$ represents the data that are used as a reference model for the simultaneous fit; Col. 3: best-fit model; Col. 4-9: parameters in the model, where $N_{\mathrm{H}}$ are in units of $10^{22} \mathrm{~cm}^{-2}$, and Col. 10: $\chi^{2} /$ d.o.f., and in SMFx (where $x=1,2$ ), the result of the F-test is presented in the second line. (†) The spectral index at hard energies is reported in Table A.7 for Compton-thick candidates. 
Table A.2. continued.

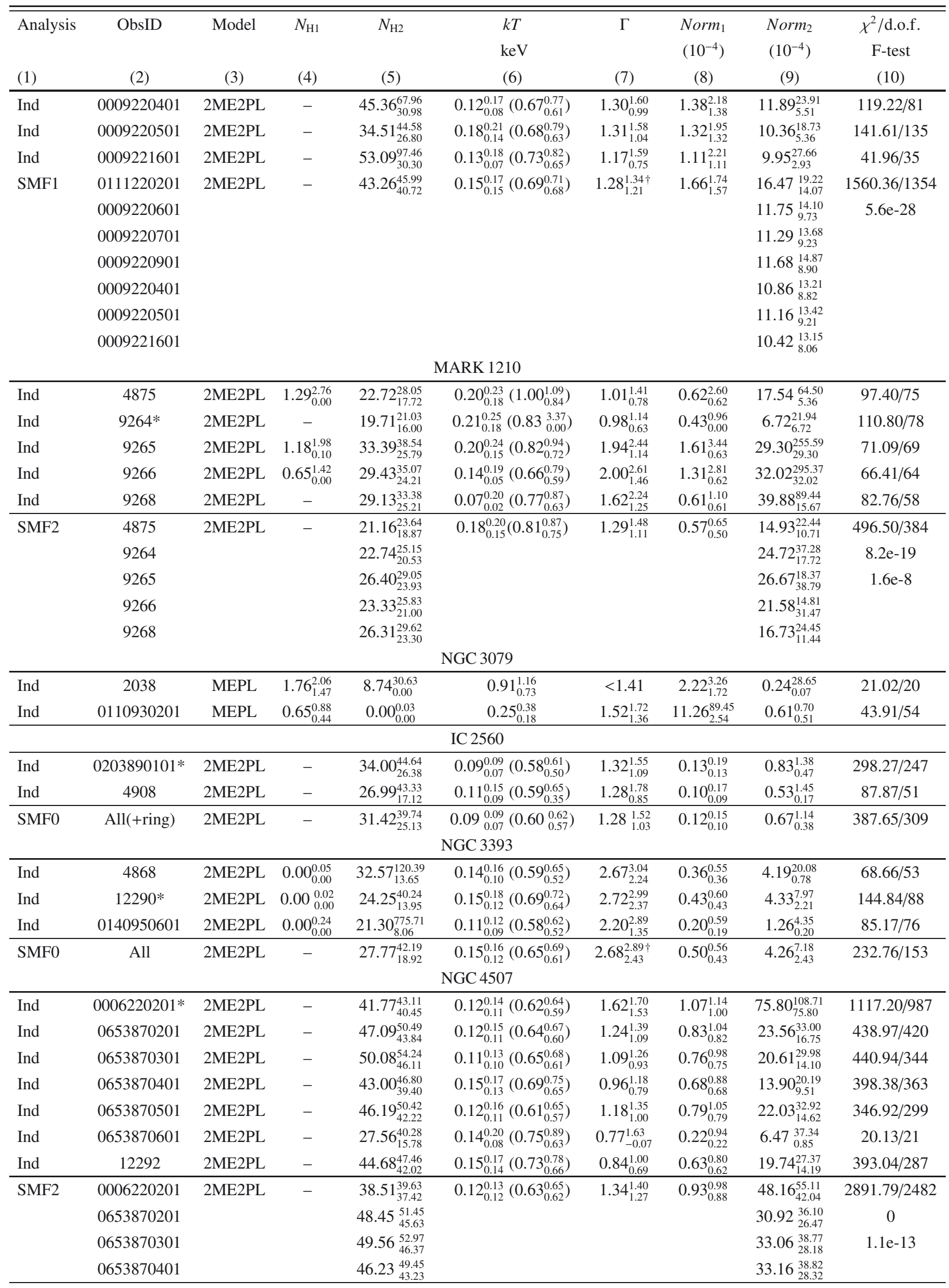


Table A.2. continued.

\begin{tabular}{|c|c|c|c|c|c|c|c|c|c|}
\hline Analysis & ObsID & Model & (4) & $N_{\mathrm{H} 2}$ & $\begin{array}{l}k T \\
\mathrm{keV} \\
(6) \\
\end{array}$ & (7) & $\begin{array}{c}\text { Norm }_{1} \\
\left(10^{-4}\right) \\
(8) \\
\end{array}$ & $\begin{array}{c}\text { Norm }_{2} \\
\left(10^{-4}\right) \\
(9)\end{array}$ & $\begin{array}{c}\chi^{2} / \text { d.o.f. } \\
\text { F-test } \\
(10)\end{array}$ \\
\hline \multirow{3}{*}{ (1) } & 0653870501 & & & $46.52 \underset{43.26}{50.03}$ & & & & $30.99 \underset{26.38}{36.41}$ & \\
\hline & 0653870601 & & & 37.0147 .70 & & & & $23.42 \begin{array}{l}31.84 \\
17.47\end{array}$ & \\
\hline & & & & & NGC 4698 & & & & \\
\hline \multirow[t]{2}{*}{ Ind } & 0112551101 & PL & $0.00_{0.00}^{0.03}$ & - & - & $2.16_{1.98}^{2.41}$ & - & - & $16.12 / 11$ \\
\hline & & $2 \mathrm{PL}$ & - & $7.45_{2.20}^{17.35}$ & - & $2.44_{2.21}^{2.80}$ & $0.20_{0.17}^{0.23}$ & $0.75_{0.21}^{1.25}$ & $8.01 / 9$ \\
\hline Ind & $0651360401 *$ & $2 \mathrm{PL}$ & - & $11.11_{6.99}^{16.21}$ & - & $2.13_{2.00}^{2.27}$ & $0.22_{0.21}^{0.23}$ & $0.74_{0.48}^{1.02}$ & $75.70 / 53$ \\
\hline SMF0 & All & $2 \mathrm{PL}$ & - & $9.84_{6.69}^{14.24}$ & & $2.19_{2.08}^{2.33}$ & $0.22_{0.21}^{0.23}$ & $0.70_{0.50}^{0.97}$ & $92.73 / 70$ \\
\hline \multicolumn{10}{|c|}{ NGC 5194} \\
\hline Ind & 1622 & ME2PL & $0.01_{0.00}^{0.26}$ & $10.42_{0.00}^{100.05}$ & $0.64_{0.57}^{0.70}$ & $2.68_{2.23}^{3.01}$ & $0.00_{0.00}^{0.23}$ & $0.22_{0.00}^{493.63}$ & $10.38 / 8$ \\
\hline Ind & 3932 & ME2PL & $0.00_{0.00}^{0.10}$ & $36.13_{24.54}^{152.06}$ & $0.66_{0.61}^{0.70}$ & $2.32_{2.12}^{2.96}$ & $0.08_{0.08}^{0.12}$ & $0.97_{0.22}^{3.67}$ & $35.93 / 27$ \\
\hline Ind & 13813 & ME2PL & - & $60.36_{27.81}^{98.94}$ & $0.64_{0.61}^{0.66}$ & $1.92 \begin{array}{l}2.10 \\
1.72\end{array}$ & $0.06_{0.06}^{0.08}$ & $0.90_{0.37}^{2.12}$ & $62.16 / 70$ \\
\hline Ind & 13812 & ME2PL & $0.14_{0.00}^{0.28}$ & $24.53_{15.73}^{123.81}$ & $0.65_{0.61}^{0.68}$ & $3.044_{2.05}^{3.87}$ & $0.08_{0.08}^{0.24}$ & $1.91_{0.53}^{6.69}$ & $83.06 / 65$ \\
\hline Ind & $13814 *$ & ME2PL & $0.06_{0.00}^{0.16}$ & $41.09_{30.76}^{56.65}$ & $0.62_{0.59}^{0.64}$ & $2.52 \begin{array}{l}3.10 \\
2.07\end{array}$ & $0.06_{0.06}^{0.13}$ & $1.91_{0.71}^{5.31}$ & $87.12 / 68$ \\
\hline Ind & 13815 & ME2PL & $0.01_{0.00}^{0.11}$ & $70.97_{35.84}^{96.19}$ & $0.67_{0.63}^{0.72}$ & $2.40_{2.23}^{3.10}$ & $0.09_{0.09}^{0.18}$ & $3.40_{0.06}^{17.48}$ & $20.00 / 31$ \\
\hline Ind & 13816 & ME2PL & $0.00_{0.00}^{0.20}$ & $152.21_{67.83}^{201.29}$ & $0.60_{0.56}^{0.64}$ & $2.06_{1.39}^{3.36}$ & $0.06_{0.06}^{0.09}$ & $11.58_{1.16}^{96.47}$ & $55.55 / 29$ \\
\hline Ind & 0112840201 & 2ME2PL & $0.00_{0.00}^{0.04}$ & $12.01_{9.82}^{14.77}$ & $0.15_{0.12}^{0.19}\left(0.60_{0.59}^{0.61}\right)$ & $2.81_{2.59}^{3.08}$ & $0.70_{0.70}^{0.94}$ & $8.69_{5.82}^{14.93}$ & $177.67 / 207$ \\
\hline SMF0 & All & ME2PL & $0.00_{0.00}^{0.05}$ & $48.69_{35.14}^{68.60}$ & $0.64_{0.63}^{0.65}$ & $2.14_{2.08}^{2.43 \dagger}$ & $0.08_{0.07}^{0.09}$ & $1.03_{0.70}^{1.81}$ & $448.67 / 358$ \\
\hline \multicolumn{10}{|c|}{ MARK 268} \\
\hline \multirow[t]{2}{*}{ Ind } & 0554500701 & $2 \mathrm{PL}$ & $0.10_{0.00}^{0.24}$ & $35.25_{28.41}^{43.55}$ & - & $2.49_{1.83}^{3.26}$ & $0.47_{0.31}^{0.74}$ & $91.33_{26.18}^{409.21}$ & $12.07 / 17$ \\
\hline & & ME2PL & $0.13_{0.00}^{1.76}$ & $31.86_{24.47}^{45.21}$ & $0.57_{0.32}^{0.88}$ & $2.11_{1.35}^{3.07}$ & $0.15_{0.15}^{1.70}$ & $42.96_{10.25}^{311.67}$ & $6.52 / 15$ \\
\hline Ind & 0554501101* & ME2PL & $0.01_{0.00}^{0.09}$ & $34.27_{30.47}^{39.16}$ & $0.81_{0.69}^{0.89}$ & $1.70_{1.48}^{2.05}$ & $0.22_{0.18}^{0.30}$ & $11.84_{11.83}^{39.96}$ & $104.44 / 99$ \\
\hline SMF0 & All & ME2PL & $0.02_{0.00}^{0.09}$ & $33.65_{30.26}^{37.93}$ & $0.78_{0.64}^{0.86}$ & $1.75_{1.51}^{2.06}$ & $0.24_{0.19}^{0.31}$ & $20.82_{12.52}^{40.42}$ & $124.87 / 124$ \\
\hline \multicolumn{10}{|c|}{ MARK 273} \\
\hline \multirow[t]{2}{*}{ Ind } & $0101640401^{*}$ & ME2PL & - & $58.46_{43.97}^{75.12}$ & $0.67_{0.61}^{0.74}$ & $1.91_{1.74}^{2.07}$ & $0.27_{0.27}^{0.34}$ & $6.19_{3.73}^{9.71}$ & $81.61 / 64$ \\
\hline & & 2ME2PL & - & $59.94_{43.57}^{78.44}$ & $0.26_{0.16}^{0.40}\left(0.74_{0.64}^{0.88}\right)$ & $1.66_{1.32}^{1.93}$ & $0.19_{0.19}^{0.31}$ & $3.78_{1.63}^{7.18}$ & $77.53 / 62$ \\
\hline Ind & 809 & 2ME2PL & $0.42_{0.17}^{1.10}$ & $45.11_{39.00}^{52.33}$ & $0.04_{0.02}^{0.16}\left(0.81_{0.73}^{0.94}\right)$ & $2.30_{1.76}^{3.07}$ & $0.26_{0.26}^{0.70}$ & $20.70_{8.06}^{107.25}$ & $56.07 / 58$ \\
\hline \multirow[t]{3}{*}{ SMF1 } & 0101640401 & 2ME2PL & - & $78.85_{67.72}^{90.96}$ & $0.01_{0.01}^{0.15}\left(0.88_{0.82}^{0.99}\right)$ & $1.33_{1.17}^{1.46}$ & $0.17_{0.15}^{0.19}$ & $2.86_{2.09}^{3.85}$ & $193.34 / 131$ \\
\hline & 809 & & & $38.43_{33.42}^{43.94}$ & & & & & \\
\hline & & & & & Circinus & & & & \\
\hline Ind & 365 & 2ME2PL & - & $60.04_{33.78}^{81.16}$ & $1.05_{0}^{0}\left(0.44_{0}^{0}\right)$ & $0.98_{0.75}^{1.23}$ & $2.45_{2.45}^{3.51}$ & $14.15_{5.09}^{25.59}$ & $130.43 / 58$ \\
\hline Ind & $9140 *$ & 2ME2PL & - & $41.33_{36.55}^{46.73}$ & $0.07_{0.03}^{0.34}\left(0.71_{0.64}^{0.77}\right)$ & $1.00_{0.91}^{1.10}$ & $2.76_{2.76}^{3.20}$ & $13.34_{10.80}^{16.57}$ & $965.62 / 397$ \\
\hline Ind & 10937 & 2ME2PL & - & $54.02_{43.53}^{67.25}$ & $0.07_{0}^{0}\left(0.75_{0.63}^{0.94}\right)$ & $0.88_{0.70}^{1.01}$ & $2.29_{2.29}^{2.93}$ & $15.15_{10.62}^{21.23}$ & $476.34 / 198$ \\
\hline Ind & $0111240101 *$ & 2ME2PL & - & $39.33_{35.06}^{44.20}$ & $0.11_{0.10}^{0.13}\left(0.59_{0.57}^{0.61}\right)$ & $1.35_{1.33}^{1.38}$ & $14.69_{14.69}^{15.40}$ & $14.52_{12.72}^{16.47}$ & $2661.99 / 1584$ \\
\hline Ind & 0656580601 & 2ME2PL & - & $46.28_{44.78}^{49.26}$ & $0.02_{0.01}^{0.40}\left(0.61_{0.59}^{0.63}\right)$ & $1.31_{1.30}^{1.33}$ & $9.43_{9.43}^{9.69}$ & $40.08_{34.17}^{46.79}$ & $2193.02 / 1090$ \\
\hline SMF0 & All (Chandra) & 2ME2PL & - & $39.70_{34.50}^{46.17}$ & $0.14_{0.04}^{0.227}\left(0.72_{0.65}^{0.84}\right)$ & $0.633_{0.53}^{0.73 \dagger}$ & $1.86_{1.69}^{2.03}$ & $5.70_{4.42}^{7.20}$ & $1114.28 / 673$ \\
\hline \multirow[t]{4}{*}{ SMF2 } & 0111240101 & 2ME2PL & - & $42.56_{39.17}^{46.27}$ & $0.11_{0.08}^{0.12}\left(0.62_{0.60}^{0.63}\right)$ & $1.34_{1.31}^{1.37}$ & $14.35_{14.03}^{14.68}$ & $15.79_{14.06}^{17.63}$ & $4410.83 / 2682$ \\
\hline & 0656580601 & & & & & & 9.489 .75 & $22.93 \underset{20.90}{25.11}$ & $1.2 \mathrm{e}-255$ \\
\hline & & & & & & & & & $1.8 \mathrm{e}-13$ \\
\hline & & & & & NGC 5643 & & & & \\
\hline Ind & 0140950101 & 2ME2PL & - & $87.04_{37.68}^{221.48}$ & $0.16_{0.14}^{0.19}\left(0.68_{0.62}^{0.77}\right)$ & $0.99_{0.60}^{1.42}$ & $0.20_{0.20}^{0.41}$ & $1.90_{0.37}^{11.03}$ & $35.98 / 46$ \\
\hline Ind & $0601420101 *$ & 2ME2PL & - & $35.23_{25.63}^{51.46}$ & $0.09_{0.08}^{0.11}\left(0.59_{0.51}^{0.63}\right)$ & $1.51_{1.21}^{1.82}$ & $0.41_{0.41}^{0.63}$ & $2.81_{1.40}^{5.35}$ & $184.91 / 139$ \\
\hline \multirow[t]{2}{*}{ SMF0 } & All & 2ME2PL & - & $44.65_{30.74}^{68.03}$ & $0.11_{0.09}^{0.14}\left(0.61_{0.58}^{0.64}\right)$ & $1.32_{1.08}^{1.59 \dagger}$ & $0.43_{0.36}^{0.52}$ & $2.26_{1.21}^{4.01}$ & $245.69 / 197$ \\
\hline & & & & & MARK 477 & & & & \\
\hline Ind & $0651100301 *$ & 2ME2PL & - & $25.58_{20.04}^{31.53}$ & $0.15_{0.11}^{0.19}\left(0.59_{0.36}^{0.74}\right)$ & $1.10_{0.71}^{1.48}$ & $0.21_{0.21}^{0.36}$ & $2.89_{1.17}^{6.60}$ & $55.96 / 63$ \\
\hline Ind & 0651100401 & 2ME2PL & - & $29.72_{24.41}^{35.63}$ & $0.11_{0.07}^{0.17}\left(0.50_{0.30}^{0.69}\right)$ & $1.54_{1.12}^{1.94}$ & $0.28_{0.28}^{0.49}$ & $8.42_{3.64}^{18.78}$ & $50.90 / 60$ \\
\hline
\end{tabular}


Table A.2. continued.

\begin{tabular}{|c|c|c|c|c|c|c|c|c|c|}
\hline Analysis & ObsID & Model & $N_{\mathrm{H} 1}$ & $N_{\mathrm{H} 2}$ & $\begin{array}{l}k T \\
\mathrm{keV} \\
(6)\end{array}$ & (7) & $\begin{array}{c}\text { Norm }_{1} \\
\left(10^{-4}\right) \\
(8)\end{array}$ & $\begin{array}{c}\mathrm{Norm}_{2} \\
\left(10^{-4}\right) \\
(9)\end{array}$ & $\begin{array}{c}\chi^{2} / \text { d.o.f. } \\
\text { F-test } \\
(10)\end{array}$ \\
\hline SMF0 & All & 2ME2PL & - & $27.76_{23.92}^{31.89}$ & $\begin{array}{l}0.15_{0.09}^{0.17}\left(0.60_{0.33}^{0.69}\right) \\
\text { IC } 4518 \mathrm{~A}\end{array}$ & $1.30_{1.02}^{1.58 \dagger}$ & $0.32_{0.26}^{0.38}$ & $4.89_{2.63}^{8.84}$ & $122.47 / 135$ \\
\hline Ind & 0401790901 & $\begin{array}{l}\text { ME2PL } \\
\text { 2ME2PL }\end{array}$ & $\begin{array}{c}- \\
0.58_{0.01}^{1.42}\end{array}$ & $\begin{array}{l}22.85_{19.99}^{25.65} \\
21.92_{19.79}^{26.44}\end{array}$ & $\begin{array}{c}0.68_{0.61}^{0.75} \\
0.18_{0.12}^{0.22}\left(0.71_{0.65}^{0.79}\right)\end{array}$ & $\begin{array}{l}1.94_{1.66}^{2.19} \\
1.72_{1.29}^{2.21}\end{array}$ & $\begin{array}{l}0.28_{0.28}^{0.42} \\
0.23_{0.23}^{0.76}\end{array}$ & $\begin{array}{l}23.18_{13.11}^{38.48} \\
15.05_{6.64}^{36.19}\end{array}$ & $\begin{array}{l}84.82 / 84 \\
69.71 / 82\end{array}$ \\
\hline Ind & 0406410101* & 2ME2PL & - & $24.65_{21.58}^{27.85}$ & $0.09_{0.05}^{0.21}\left(0.65_{0.60}^{0.69}\right)$ & $1.47_{1.21}^{1.72}$ & $0.22_{0.22}^{0.33}$ & $6.05_{3.46}^{10.42}$ & $169.18 / 161$ \\
\hline \multirow[t]{2}{*}{ SMF1 } & $\begin{array}{l}0401790901 \\
0406410101\end{array}$ & 2ME2PL & - & $22.22_{19.98}^{24.52}$ & $0.16_{0.09}^{0.22}\left(0.68_{0.64}^{0.76}\right)$ & $1.50_{1.29}^{1.70}$ & $0.27_{0.22}^{0.31}$ & $\begin{array}{c}10.29_{6.70}^{15.68} \\
5.92_{3.83}^{9.08}\end{array}$ & $\begin{array}{c}258.87 / 254 \\
5.2 \mathrm{e}-26\end{array}$ \\
\hline & \multicolumn{9}{|c|}{ ESO 138-G01 } \\
\hline Ind & $0405380201 *$ & ME2PL & - & $31.43_{27.61}^{35.98}$ & $0.71_{0.65}^{0.78}$ & $2.31_{2.21}^{2.40}$ & $1.70_{1.70}^{1.94}$ & $38.74_{30.65}^{48.57}$ & $287.95 / 174$ \\
\hline Ind & 0690580101 & ME2PL & - & $25.88_{22.91}^{30.83}$ & $0.71_{0.66}^{0.84}$ & $2.60_{2.49}^{2.71}$ & $1.76_{1.76}^{2.04}$ & $49.99_{39.89}^{66.24}$ & $211.76 / 126$ \\
\hline SMF0 & All & ME2PL & - & $29.82_{26.29}^{32.26}$ & $0.71_{0.68}^{0.77}$ & $2.42_{2.35}^{2.50 \dagger}$ & $1.85_{1.75}^{1.94}$ & $43.38_{36.05}^{51.19}$ & $521.97 / 310$ \\
\hline \multicolumn{10}{|c|}{ NGC 6300} \\
\hline Ind & $10292 *$ & $2 \mathrm{PL}$ & $0.00_{0.00}^{0.42}$ & $14.06_{12.14}^{15.37}$ & - & $0.77_{0.42}^{0.99}$ & $0.12_{0.08}^{0.16}$ & $17.51_{10.86}^{29.15}$ & $145.61 / 131$ \\
\hline Ind & 10293 & $2 \mathrm{PL}$ & - & $19.83_{17.11}^{21.17}$ & - & $1.48_{1.07}^{1.69}$ & $0.13_{0.10}^{0.17}$ & $76.69_{35.41}^{113.25}$ & $130.28 / 121$ \\
\hline Ind & 0059770101 & $2 \mathrm{PL}$ & - & $25.41_{21.70}^{29.69}$ & - & $2.19_{1.78}^{2.65}$ & $0.02_{0.01}^{0.02}$ & $5.64_{2.52}^{13.89}$ & $29.26 / 35$ \\
\hline SMF0 & All & $2 \mathrm{PL}$ & - & $16.46_{15.32}^{17.85}$ & - & $1.02_{0.86}^{1.26}$ & $0.12_{0.10}^{0.15}$ & $32.70_{24.19}^{51.16}$ & $304.19 / 260$ \\
\hline \multicolumn{10}{|c|}{ NGC 7172} \\
\hline Ind & 0147920601 & ME2PL & $0.00_{0.00}^{0.05}$ & $8.45_{8.12}^{8.81}$ & $0.35_{0.27}^{0.57}$ & $1.52_{1.44}^{1.58}$ & $0.23_{0.23}^{0.33}$ & $61.18_{53.78}^{69.78}$ & $655.26 / 682$ \\
\hline Ind & 0202860101 & ME2PL & - & $8.75_{8.48}^{9.02}$ & $0.20_{0.17}^{0.25}$ & $1.56_{1.50}^{1.62}$ & $0.30_{0.27}^{0.34}$ & $57.43_{57.43}^{70.75}$ & $943.47 / 1046$ \\
\hline Ind & $0414580101^{*}$ & ME2PL & - & $8.34_{8.19}^{8.50}$ & $0.28_{0.19}^{0.51}$ & $1.65_{1.61}^{1.68}$ & $0.31_{0.31}^{0.39}$ & $152.90_{143.87}^{162.22}$ & $1482.81 / 1454$ \\
\hline \multirow[t]{4}{*}{ SMF1 } & 0147920601 & ME2PL & - & $8.43_{8.31}^{8.56}$ & $0.26_{0.24}^{0.31}$ & $1.61_{1.58}^{1.63}$ & $0.30_{0.28}^{0.32}$ & $70.73_{67.35}^{74.30}$ & $3198.23 / 3200$ \\
\hline & 0202860101 & & & & & & & $66.95_{63.78}^{70.30}$ & 0 \\
\hline & 0414580101 & & & & & & & $145.45_{138.64}^{152.64}$ & \\
\hline & \multicolumn{9}{|c|}{ NGC 7212} \\
\hline Ind & $0200430201^{*}$ & 2ME2PL & - & $118.96_{54.65}^{264.77}$ & $0.16_{0.11}^{0.20}\left(0.66_{0.58}^{0.78}\right)$ & $0.64_{0.39}^{0.91}$ & $0.15_{0.15}^{0.25}$ & $1.42_{0.28}^{8.63}$ & $57.77 / 46$ \\
\hline Ind & 4078 & 2ME2PL & - & $34.50_{28.60}^{46.59}$ & $0.01_{0.00}^{0.83}\left(0.49_{0.26}^{0.65}\right)$ & $1.22_{1.14}^{1.36}$ & $0.19_{0.19}^{0.24}$ & $2.66_{0.94}^{7.05}$ & $45.19 / 19$ \\
\hline SMF0 & All(+ring) & 2ME2PL & - & $81.67_{51.53}^{124.96}$ & $\begin{array}{l}0.12_{0.08}^{0.17}\left(0.59_{0.52}^{0.66}\right) \\
\text { NGC } 7319\end{array}$ & $0.80_{0.57}^{1.04 \dagger}$ & $0.17_{0.14}^{0.21}$ & $1.43_{0.62}^{2.92}$ & $106.34 / 77$ \\
\hline Ind & 789 & ME2PL & - & $39.11_{33.65}^{48.43}$ & $0.85_{0.73}^{0.99}$ & $1.29_{0.79}^{2.20}$ & $0.08_{0.05}^{0.14}$ & $4.42_{4.42}^{52.91}$ & $47.441 / 31$ \\
\hline Ind & $7924^{*}$ & ME2PL & - & $46.06_{43.15}^{49.01}$ & $0.65_{0.57}^{0.75}$ & $2.03_{1.79}^{2.25}$ & $0.19_{0.16}^{0.22}$ & $40.65_{40.65}^{10.32}$ & $187.45 / 141$ \\
\hline Ind & 0021140201 & 2ME2PL & - & $51.88_{46.20}^{57.69}$ & $0.18_{0.08}^{0.24}\left(0.63_{0.60}^{0.67}\right)$ & $1.35_{1.07}^{1.63}$ & $0.12_{0.11}^{0.18}$ & $7.17_{3.79}^{13.38}$ & $263.86 / 213$ \\
\hline \multirow[t]{2}{*}{ SMF2 } & 789 & ME2PL & $0.65_{0.36}^{0.99}$ & $46.87_{44.36}^{49.70}$ & $0.67_{0.60}^{0.81}$ & $2.03_{1.88}^{2.29}$ & $0.19_{0.17}^{0.22}$ & $41.72_{29.64}^{70.42}$ & $240.07 / 193$ \\
\hline & 7924 & & - & & & & & $67.87_{49.42}^{113.69}$ & $\begin{array}{c}1.5 \mathrm{e}-18 \\
1.3 \mathrm{e}-9\end{array}$ \\
\hline
\end{tabular}


Table A.3. X-ray luminosities.

\begin{tabular}{|c|c|c|c|c|c|c|}
\hline \multirow{3}{*}{$\begin{array}{c}\text { Name } \\
\text { (1) }\end{array}$} & \multirow{3}{*}{$\begin{array}{c}\text { Satellite } \\
\text { (2) }\end{array}$} & \multirow{3}{*}{$\begin{array}{l}\text { ObsID } \\
\text { (3) }\end{array}$} & \multicolumn{2}{|c|}{ Individual } & \multicolumn{2}{|c|}{ Simultaneous } \\
\hline & & & $\log (L(0.5-2 \mathrm{keV}))$ & $\log (L(2-10 \mathrm{keV}))$ & $\log (L(0.5-2 \mathrm{keV}))$ & $\log (L(2-10 \mathrm{keV}))$ \\
\hline & & & (4) & $(5)$ & (6) & (7) \\
\hline \multirow[t]{2}{*}{ MARK 348} & XMM-Newton & 0067540201 & $43.01_{42.98}^{43.03}$ & $43.41_{43.40}^{43.41}$ & $43.01_{42.99}^{43.02}$ & $43.40_{43.40}^{43.41}$ \\
\hline & XMM-Newton & 0701180101 & $42.47_{42.44}^{42.50}$ & $42.89_{42.88}^{42.91}$ & $42.50_{42.49}^{42.52}$ & $42.90_{42.89}^{42.91}$ \\
\hline \multirow[t]{4}{*}{ NGC 424} & XMM-Newton & 0002942301 & $41.70_{41.68}^{41.72}$ & $42.01_{41.93}^{42.07}$ & $41.74_{41.72}^{41.76}$ & $41.87_{41.81}^{41.92}$ \\
\hline & XMM-Newton & 0550950101 & $41.95_{41.94}^{41.95}$ & $42.00_{41.99}^{42.01}$ & & \\
\hline & Chandra $\left(2^{\prime \prime}\right)$ & 3146 & $42.02_{41.97}^{42.06}$ & $41.91_{41.83}^{41.97}$ & $41.74_{41.72}^{41.76}$ & $41.84_{41.79}^{41.89}$ \\
\hline & Chandra $\left(20^{\prime \prime}\right)$ & 3146 & $42.00_{41.95}^{42.04}$ & $41.94_{41.86}^{42.01}$ & & \\
\hline \multirow[t]{4}{*}{ MARK 573} & Chandra $\left(2^{\prime \prime}\right)$ & 7745 & $42.02_{41.98}^{42.06}$ & $41.69_{41.59}^{41.77}$ & $41.65_{41.56}^{41.72}$ & $41.54_{41.48}^{41.59}$ \\
\hline & Chandra $\left(20^{\prime \prime}\right)$ & 7745 & $42.19_{42.17}^{42.21}$ & $41.87_{41.76}^{41.96}$ & & \\
\hline & Chandra & 13124 & $41.94_{41.89}^{41.98}$ & $41.73_{41.66}^{41.80}$ & $41.64_{41.55}^{41.71}$ & $41.54_{41.49}^{41.59}$ \\
\hline & XMM-Newton & 0200430701 & $42.23_{42.21}^{42.25}$ & $41.61_{41.55}^{41.66}$ & & \\
\hline \multirow[t]{3}{*}{ NGC 788} & XMM-Newton & 0601740201 & $42.23_{42.21}^{42.25}$ & $42.67_{42.64}^{42.70}$ & $42.11_{42.04}^{42.17}$ & $42.63_{42.60}^{42.66}$ \\
\hline & Chandra $\left(3^{\prime \prime}\right)$ & 11680 & $41.70_{41.66}^{41.74}$ & $42.59_{42.42}^{42.71}$ & $42.11_{42.04}^{42.17}$ & $42.59 \begin{array}{l}42.62 \\
42.56\end{array}$ \\
\hline & Chandra $\left(20^{\prime \prime}\right)$ & 11680 & $41.81_{41.76}^{41.85}$ & $42.70_{42.55}^{42.82}$ & & \\
\hline \multirow[t]{2}{*}{ ESO 417-G06 } & XMM-Newton & 0602560201 & $42.59_{42.51}^{42.66}$ & $42.50_{42.48}^{42.52}$ & $42.46_{42.26}^{42.59}$ & $42.50_{42.48}^{42.52}$ \\
\hline & XMM-Newton & 0602560301 & $42.39_{42.33}^{42.44}$ & $42.51_{42.49}^{42.53}$ & $42.46_{42.21}^{42.62}$ & $42.50_{42.48}^{42.52}$ \\
\hline \multirow[t]{3}{*}{ MARK 1066} & XMM-Newton & 0201770201 & $41.79_{41.77}^{41.82}$ & $41.75_{41.70}^{41.80}$ & $41.40_{41.38}^{41.42}$ & $41.44_{41.33}^{41.53}$ \\
\hline & Chandra $\left(3^{\prime \prime}\right)$ & 4075 & $41.68_{41.60}^{41.75}$ & $41.55_{41.21}^{41.73}$ & $41.39_{41.37}^{41.41}$ & $41.42_{41.32}^{41.49}$ \\
\hline & Chandra $\left(20^{\prime \prime}\right)$ & 4075 & $41.90_{41.81}^{41.97}$ & $41.02_{40.72}^{41.19}$ & & \\
\hline \multirow[t]{4}{*}{ 3C 98.0} & XMM-Newton & 0064600101 & $43.28_{43.15}^{43.39}$ & $42.91_{42.89}^{42.92}$ & $43.14_{42.87}^{43.30}$ & $42.92_{42.89}^{42.95}$ \\
\hline & XMM-Newton & 0064600301 & $43.15_{42.96}^{43.29}$ & $42.68_{42.61}^{42.74}$ & $43.12_{42.83}^{43.29}$ & $42.68_{42.63}^{42.72}$ \\
\hline & Chandra $\left(2^{\prime \prime}\right)$ & 10234 & $42.67_{42.47}^{42.80}$ & $42.61_{42.48}^{42.70}$ & & \\
\hline & Chandra $\left(20^{\prime \prime}\right)$ & 10234 & $42.81_{42.56}^{42.97}$ & $42.64_{42.50}^{42.74}$ & & \\
\hline \multirow[t]{7}{*}{ MARK 3} & XMM-Newton & 0111220201 & $42.29_{42.29}^{42.30}$ & $42.86_{42.86}^{42.87}$ & $42.35_{42.35}^{42.36}$ & $42.87_{42.86}^{42.88}$ \\
\hline & XMM-Newton & 0009220601 & $42.16_{42.14}^{42.17}$ & $42.73_{42.70}^{42.77}$ & $42.24_{42.23}^{42.24}$ & $42.74_{42.72}^{42.76}$ \\
\hline & $X M M-N e w t o n$ & 0009220701 & $42.03_{42.01}^{42.05}$ & $42.66_{42.61}^{42.71}$ & $42.22_{42.22}^{42.23}$ & $42.73_{42.70}^{42.75}$ \\
\hline & XMM-Newton & 0009220901 & $41.76_{41.72}^{41.79}$ & $42.52_{42.43}^{42.59}$ & $42.23_{42.23}^{42.24}$ & $42.74_{42.69}^{42.79}$ \\
\hline & XMM-Newton & 0009220401 & $42.09_{42.07}^{42.11}$ & $42.72_{42.67}^{42.76}$ & $42.21_{42.21}^{42.22}$ & $42.71_{42.68}^{42.74}$ \\
\hline & XMM-Newton & 0009220501 & $42.11_{42.09}^{42.12}$ & $42.65_{42.62}^{42.68}$ & $42.22_{42.21}^{42.22}$ & $42.72_{42.70}^{42.75}$ \\
\hline & XMM-Newton & 0009221601 & $42.04_{42.01}^{42.07}$ & $42.72_{42.63}^{42.79}$ & $\begin{array}{r}42.20_{42.19}^{42.20} \\
\end{array}$ & $42.70_{42.65}^{42.74}$ \\
\hline \multirow[t]{5}{*}{ MARK 1210} & Chandra & 4875 & $42.25_{42.21}^{42.29}$ & $42.69_{42.42}^{42.85}$ & $42.19_{42.18}^{42.20}$ & $\begin{array}{l}42.67_{42.64}^{42.70} \\
\text {. }\end{array}$ \\
\hline & Chandra & 9264 & $42.36_{42.24}^{42.45}$ & $42.87_{42.73}^{42.99}$ & $42.40_{42.38}^{42.41}$ & $42.88_{42.85}^{42.91}$ \\
\hline & Chandra & 9265 & $42.89_{42.84}^{42.94}$ & $43.02_{42.74}^{43.19}$ & $42.41_{42.40}^{42.43}$ & $42.90_{42.86}^{42.93}$ \\
\hline & Chandra & 9266 & $41.43_{41.33}^{41.52}$ & $42.60_{42.58}^{42.62}$ & $42.33_{42.31}^{42.34}$ & $42.81_{42.77}^{42.84}$ \\
\hline & Chandra & 9268 & $42.57_{42.49}^{42.64}$ & $42.77_{42.53}^{42.93}$ & $42.22_{42.21}^{42.24}$ & $42.70_{42.66}^{42.74}$ \\
\hline \multirow[t]{3}{*}{ NGC 3079} & Chandra $\left(4^{\prime \prime}\right)$ & 2038 & $39.58_{39.46}^{39.67}$ & $39.82_{39.72}^{39.90}$ & & \\
\hline & Chandra $\left(25^{\prime \prime}\right)$ & 2038 & $40.02_{39.93}^{40.10}$ & $40.07_{39.99}^{40.14}$ & & \\
\hline & XMM-Newton & 0110930201 & $39.88_{39.86}^{39.90}$ & $40.08_{40.05}^{40.10}$ & & \\
\hline \multirow[t]{3}{*}{ IC 2560} & XMM-Newton & 0203890101 & $40.72_{40.71}^{40.73}$ & $41.12_{41.09}^{41.14}$ & $40.57_{40.56}^{40.58}$ & $41.05_{41.03}^{41.08}$ \\
\hline & Chandra $\left(3^{\prime \prime}\right)$ & 4908 & $40.48_{40.44}^{40.53}$ & $40.95_{40.87}^{41.02}$ & $40.56_{40.55}^{40.57}$ & $41.01_{40.99}^{41.04}$ \\
\hline & Chandra $\left(20^{\prime \prime}\right)$ & 4908 & $40.61_{40.57}^{40.64}$ & $41.01_{40.93}^{41.08}$ & & \\
\hline
\end{tabular}

Notes. Columns 4 and 5: soft and hard intrinsic luminosities for individual fits; Cols. 6 and 7: soft and hard intrinsic luminosities for simultaneous fitting. Blanks mean observations that are not used for the simultaneous fittings. 
Table A.3. continued.

\begin{tabular}{|c|c|c|c|c|c|c|}
\hline \multirow{3}{*}{$\begin{array}{c}\text { Name } \\
(1)\end{array}$} & \multirow{3}{*}{$\begin{array}{c}\text { Satellite } \\
\text { (2) }\end{array}$} & \multirow{3}{*}{$\begin{array}{l}\text { ObsID } \\
(3)\end{array}$} & \multicolumn{2}{|c|}{ Individual } & \multicolumn{2}{|c|}{ Simultaneous } \\
\hline & & & $\log (L(0.5-2 \mathrm{keV}))$ & $\log (L(2-10 \mathrm{keV}))$ & $\log (L(0.5-2 \mathrm{keV}))$ & $\log (L(2-10 \mathrm{keV}))$ \\
\hline & & & (4) & $(5)$ & (6) & (7) \\
\hline \multirow[t]{4}{*}{ NGC 3393} & Chandra $\left(5^{\prime \prime}\right)$ & 4868 & $41.63_{41.61}^{41.65}$ & $41.29_{41.14}^{41.40}$ & $41.64_{41.63}^{41.65}$ & $41.29_{41.25}^{41.32}$ \\
\hline & Chandra $\left(20^{\prime \prime}\right)$ & 4868 & $41.59_{41.57}^{41.61}$ & $41.28_{41.17}^{41.36}$ & & \\
\hline & Chandra & 12290 & $41.65_{41.64}^{41.67}$ & $41.29_{41.24}^{41.33}$ & $41.64_{41.63}^{41.65}$ & $41.29_{41.25}^{41.32}$ \\
\hline & XMM-Newton & 0140950601 & $41.38_{41.36}^{41.39}$ & $41.21_{40.97}^{41.37}$ & & \\
\hline \multirow[t]{8}{*}{ NGC 4507} & XMM-Newton & 0006220201 & $42.79_{42.78}^{42.79}$ & $43.12_{43.12}^{43.13}$ & $42.55_{42.55}^{42.56}$ & $43.05_{43.05}^{43.06}$ \\
\hline & $X M M-N e w t o n$ & 0653870201 & $42.16_{42.15}^{42.17}$ & $42.82_{42.81}^{42.83}$ & $41.19_{41.19}^{41.20}$ & $42.34_{42.33}^{42.35}$ \\
\hline & XMM-Newton & 0653870301 & $42.20_{42.19}^{42.21}$ & $42.87_{42.85}^{42.88}$ & $42.40_{42.39}^{42.40}$ & $42.90_{42.88}^{42.91}$ \\
\hline & XMM-Newton & 0653870401 & $42.04_{42.03}^{42.05}$ & $42.81_{42.80}^{42.82}$ & $41.06_{41.05}^{41.06}$ & $42.39_{42.37}^{42.40}$ \\
\hline & XMM-Newton & 0653870501 & $42.20_{42.18}^{42.21}$ & $42.84_{42.82}^{42.85}$ & $41.19_{41.19}^{41.20}$ & $42.35_{42.34}^{42.37}$ \\
\hline & XMM-Newton & 0653870601 & $41.65_{41.61}^{41.69}$ & $42.61_{42.45}^{42.72}$ & $41.19_{41.19}^{41.20}$ & $42.33_{42.28}^{42.37}$ \\
\hline & Chandra $\left(2^{\prime \prime}\right)$ & 12292 & $42.21_{42.20}^{42.23}$ & $42.99 \begin{array}{l}43.01 \\
42.97\end{array}$ & & \\
\hline & Chandra $\left(30^{\prime \prime}\right)$ & 12292 & $42.24_{42.22}^{42.25}$ & $43.07 \begin{array}{r}43.09 \\
43.05\end{array}$ & & \\
\hline \multirow[t]{2}{*}{ NGC 4698} & XMM-Newton & 0112551101 & $40.00_{39.95}^{40.05}$ & $39.97_{39.78}^{42.91}$ & $40.14_{40.12}^{40.17}$ & $40.08_{40.04}^{40.12}$ \\
\hline & XMM-Newton & 0651360401 & $40.22_{40.19}^{40.24}$ & $40.16_{39.73}^{41.22}$ & $40.14_{40.12}^{40.17}$ & $40.08_{40.04}^{40.12}$ \\
\hline \multirow[t]{9}{*}{ NGC 5194} & Chandra & 1622 & $39.25_{39.15}^{39.34}$ & $38.88_{38.33}^{39.12}$ & $39.53_{39.52}^{39.54}$ & $39.51_{39.48}^{39.54}$ \\
\hline & Chandra $\left(2^{\prime \prime}\right)$ & 3932 & $39.30_{39.25}^{39.35}$ & $39.21_{39.07}^{39.32}$ & $39.53_{39.52}^{39.54}$ & $39.51_{39.48}^{39.54}$ \\
\hline & Chandra $\left(25^{\prime \prime}\right)$ & 3932 & $40.26_{40.24}^{40.28}$ & $39.64_{39.50}^{39.74}$ & & \\
\hline & Chandra & 13813 & $39.26_{39.24}^{39.27}$ & $39.34_{39.26}^{39.41}$ & $39.53_{39.52}^{39.54}$ & $39.51_{39.48}^{39.54}$ \\
\hline & Chandra & 13812 & $40.33_{40.29}^{40.36}$ & $40.39_{40.23}^{40.51}$ & $39.53_{39.52}^{39.54}$ & $39.51_{39.48}^{39.54}$ \\
\hline & Chandra & 13814 & $39.57_{39.55}^{39.60}$ & $39.46_{39.34}^{39.56}$ & $39.53_{39.52}^{39.54}$ & $39.51_{39.48}^{39.54}$ \\
\hline & Chandra & 13815 & $39.79_{39.75}^{39.84}$ & $39.58_{39.41}^{39.70}$ & $39.53_{39.52}^{39.54}$ & $39.51_{39.48}^{39.54}$ \\
\hline & Chandra & 13816 & $40.32_{40.30}^{40.35}$ & $40.41_{40.21}^{40.55}$ & $39.53_{39.52}^{39.54}$ & $39.51_{39.48}^{39.54}$ \\
\hline & XMM-Newton & 0112840201 & $39.46_{39.45}^{39.47}$ & $39.66_{39.65}^{39.67}$ & & \\
\hline \multirow[t]{2}{*}{ MARK 268} & XMM-Newton & 0554500701 & $41.29_{41.22}^{41.34}$ & $43.54_{43.48}^{43.60}$ & $41.45_{41.41}^{41.48}$ & $42.92_{42.84}^{42.98}$ \\
\hline & XMM-Newton & 0554501101 & $41.39_{41.37}^{41.42}$ & $43.42_{43.40}^{43.44}$ & $41.27_{41.24}^{41.30}$ & $42.92_{42.84}^{42.98}$ \\
\hline \multirow[t]{3}{*}{ MARK 273} & XMM-Newton & 0101640401 & $42.71_{42.68}^{42.73}$ & $42.83_{42.80}^{42.87}$ & $41.40_{41.39}^{41.41}$ & $42.20_{42.16}^{42.24}$ \\
\hline & Chandra $\left(4^{\prime \prime}\right)$ & 809 & $43.20_{43.16}^{43.24}$ & $43.06_{42.83}^{43.21}$ & $41.28_{41.26}^{41.30}$ & $42.37_{42.33}^{42.41}$ \\
\hline & Chandra $\left(20^{\prime \prime}\right)$ & 809 & $42.97_{42.95}^{43.00}$ & $\begin{array}{r}43.03 \quad \begin{array}{r}43.12 \\
42.93\end{array} \\
\end{array}$ & & \\
\hline \multirow[t]{6}{*}{ Circinus } & Chandra & 365 & $39.98_{39.88}^{40.06}$ & $40.76_{40.70}^{40.81}$ & $39.80_{39.78}^{39.82}$ & $40.60_{40.59}^{40.61}$ \\
\hline & Chandra & 9140 & $40.03_{40.02}^{40.05}$ & $40.66_{40.65}^{40.67}$ & $39.80_{39.78}^{39.82}$ & $40.60_{40.59}^{40.61}$ \\
\hline & Chandra (2") & 10937 & $39.98_{39.96}^{40.00}$ & $40.76_{40.74}^{40.78}$ & $39.80_{39.78}^{39.82}$ & $40.60_{40.59}^{40.61}$ \\
\hline & Chandra $\left(15^{\prime \prime}\right)$ & 10937 & $40.39_{40.38}^{40.40}$ & $40.96_{40.94}^{40.98}$ & & \\
\hline & XMM-Newton & 0111240101 & $40.49_{40.48}^{40.49}$ & $40.71_{40.71}^{40.72}$ & $40.50_{40.50}^{40.50}$ & $40.74_{40.73}^{40.74}$ \\
\hline & XMM-Newton & 0656580601 & $40.66_{40.65}^{40.66}$ & $40.83_{40.83}^{40.84}$ & $40.51_{40.51}^{40.52}$ & $40.76_{40.76}^{40.77}$ \\
\hline \multirow[t]{2}{*}{ NGC 5643} & XMM-Newton & 0601420101 & $40.51_{40.49}^{40.53}$ & $40.84_{40.80}^{40.88}$ & $40.44_{40.42}^{40.47}$ & $40.87_{40.84}^{40.90}$ \\
\hline & XMM-Newton & 0140950101 & $40.38_{40.36}^{40.41}$ & $40.98_{40.91}^{41.04}$ & $40.44_{40.42}^{40.47}$ & $40.87_{40.84}^{40.90}$ \\
\hline \multirow[t]{2}{*}{ MARK 477} & XMM-Newton & 0651100301 & $42.54_{42.51}^{42.56}$ & $43.06_{43.01}^{43.11}$ & $42.60_{42.58}^{42.62}$ & $43.11_{43.06}^{43.16}$ \\
\hline & XMM-Newton & 0651100401 & $42.94_{42.91}^{42.97}$ & $43.21_{43.16}^{43.2}$ & $42.60_{42.58}^{42.62}$ & $43.11_{43.06}^{43.16}$ \\
\hline \multirow[t]{2}{*}{ IC 4518A } & XMM-Newton & 0401790901 & $42.33_{42.30}^{42.35}$ & $42.57_{42.54}^{72.59}$ & $42.17_{42.14}^{42.19}$ & $42.56_{42.52}^{42.59}$ \\
\hline & XMM-Newton & 0406410101 & $41.92_{41.89}^{41.95}$ & $42.36_{42.33}^{42.38}$ & $41.95_{41.92}^{41.97}$ & $42.33_{42.30}^{42.36}$ \\
\hline \multirow[t]{2}{*}{ ESO 138-G01 } & XMM-Newton & 0405380201 & $42.23_{42.22}^{42.24}$ & $42.12_{42.10}^{42.14}$ & $42.23_{42.22}^{42.25}$ & $42.11_{42.09}^{42.13}$ \\
\hline & XMM-Newton & 0690580101 & $42.29_{42.27}^{42.30}$ & $42.05_{42.02}^{42.07}$ & $42.23 \begin{array}{r}42.25 \\
42.22\end{array}$ & $42.11_{42.09}^{42.13}$ \\
\hline \multirow[t]{3}{*}{ NGC 6300} & Chandra $\left(2^{\prime \prime}\right)$ & 10292 & $41.05_{40.91}^{41.15}$ & $41.92_{41.88}^{41.96}$ & $41.32_{41.23}^{41.40}$ & $41.95_{41.93}^{41.98}$ \\
\hline & Chandra $\left(20^{\prime \prime}\right)$ & 10292 & $41.17_{40.98}^{41.30}$ & $41.96_{41.91}^{42.01}$ & & \\
\hline & Chandra & 10293 & $41.64_{41.48}^{41.75}$ & $42.01_{41.95}^{42.06}$ & $41.32_{41.23}^{41.40}$ & $41.95_{41.93}^{41.98}$ \\
\hline
\end{tabular}


Table A.3. continued.

\begin{tabular}{|c|c|c|c|c|c|c|}
\hline \multirow{3}{*}{$\begin{array}{c}\text { Name } \\
\text { (1) }\end{array}$} & \multirow{3}{*}{$\begin{array}{c}\text { Satellite } \\
\text { (2) }\end{array}$} & \multirow{3}{*}{$\begin{array}{l}\text { ObsID } \\
(3)\end{array}$} & \multicolumn{2}{|c|}{ Individual } & \multicolumn{2}{|c|}{ Simultaneous } \\
\hline & & & $\log (L(0.5-2 \mathrm{keV}))$ & $\log (L(2-10 \mathrm{keV}))$ & $\log (L(0.5-2 \mathrm{keV}))$ & $\log (L(2-10 \mathrm{keV}))$ \\
\hline & & & (4) & (5) & (6) & (7) \\
\hline & XMM-Newton & 0059770101 & $40.44_{40.35}^{40.52}$ & $40.45_{40.41}^{40.48}$ & & \\
\hline \multirow[t]{3}{*}{ NGC 7172} & XMM-Newton & 0147920601 & $42.30_{42.28}^{42.32}$ & $42.67_{42.67}^{42.68}$ & $42.35_{42.33}^{42.36}$ & $42.67_{42.67}^{42.68}$ \\
\hline & XMM-Newton & 0202860101 & $42.30_{42.28}^{42.32}$ & $42.66_{42.65}^{42.66}$ & $42.32_{42.31}^{42.33}$ & $42.65_{42.64}^{42.65}$ \\
\hline & $X M M-N e w t o n$ & 0414580101 & $42.68_{42.67}^{42.69}$ & $42.98_{42.98}^{42.98}$ & $42.66_{42.65}^{42.67}$ & $42.98_{42.98}^{42.98}$ \\
\hline \multirow[t]{3}{*}{ NGC 7212} & XMM-Newton & 0200430201 & $41.65_{41.62}^{41.68}$ & $42.58_{42.54}^{42.62}$ & $41.81_{41.76}^{41.84}$ & $42.633_{42.56}^{42.69}$ \\
\hline & Chandra $\left(3^{\prime \prime}\right)$ & 4078 & $42.04_{42.00}^{42.08}$ & $42.48_{42.45}^{43.38}$ & $41.80_{41.74}^{41.84}$ & $42.58_{42.52}^{42.64}$ \\
\hline & Chandra $\left(20^{\prime \prime}\right)$ & 4078 & $42.06_{42.03}^{42.09}$ & $42.48_{42.35}^{42.57}$ & & \\
\hline \multirow[t]{4}{*}{ NGC 7319} & Chandra & 789 & $42.68_{42.62}^{42.74}$ & $42.82_{42.61}^{42.96}$ & $42.88_{42.81}^{42.94}$ & $42.87_{42.83}^{42.90}$ \\
\hline & Chandra (3") & 7924 & $43.03_{42.99}^{43.07}$ & $43.06_{42.99}^{43.12}$ & $43.09_{43.06}^{43.12}$ & $43.08_{43.05}^{43.10}$ \\
\hline & Chandra $\left(20^{\prime \prime}\right)$ & 7924 & $43.31_{43.28}^{43.33}$ & $43.15_{43.12}^{43.18}$ & & \\
\hline & XMM-Newton & 0021140201 & $42.10_{42.09}^{42.12}$ & $42.58_{42.56}^{42.60}$ & & \\
\hline
\end{tabular}


L. Hernández-García et al.: X-ray variability of Seyfert 2s

Table A.4. Results for the best fit of the annular region (ring) in Chandra data, and the best fit obtained for the nucleus of XMM-Newton data when the contribution from the annular region was removed.

\begin{tabular}{|c|c|c|c|c|c|c|c|c|c|c|}
\hline Name (obsID) & Region & Model & $N_{\mathrm{H} 1}^{a}$ & $N_{\mathrm{H} 2}^{a}$ & $\begin{array}{c}k T \\
(\mathrm{keV})\end{array}$ & $\Gamma$ & $\chi_{\mathrm{r}}^{2}$ & $\begin{array}{c}\log \left(L_{\text {soft }}\right) \\
(0.5-2 \mathrm{keV})\end{array}$ & $\begin{array}{c}\log \left(L_{\text {hard }}\right) \\
(2-10 \mathrm{keV})\end{array}$ & $\begin{array}{c}\text { Cont. } \\
\%\end{array}$ \\
\hline (1) & (2) & (3) & (4) & (5) & $(6)$ & (7) & $(8)$ & (9) & $(10)$ & $(11)$ \\
\hline MARK 573 (7745) & Ring* & ME2PL & - & $75.90_{26.64}^{160.34}$ & $0.67_{0.57}^{0.75}$ & $3.86_{3.64}^{-}$ & 1.97 & 43.12 & 42.01 & 24 \\
\hline MARK 573 (0200430701) & Nucleus** & 2ME2PL & - & $9.82_{5.83}^{18.25}$ & $0.15_{0.14}^{0.18}\left(0.76_{0.68}^{0.86}\right)$ & $3.01_{2.61}^{3.37}$ & 0.86 & 41.88 & 41.34 & - \\
\hline MARK 1066 (4075) & Ring* & PL & $0.21_{0.00}^{0.35}$ & - & - & $3.99_{2.68}^{4.00}$ & 1.40 & 39.65 & 39.26 & 8 \\
\hline MARK 1066 (0201770201) & Nucleus** & ME2PL & $0.08_{0.00}^{0.16}$ & $53.37_{28.95}^{110.46}$ & $0.76_{0.61}^{0.87}$ & $2.00_{1.50}^{2.56}$ & 0.78 & 41.61 & 41.67 & - \\
\hline 3C $98.0(10234)$ & Ring* & $\mathrm{ME}$ & $15.78_{8.21}^{27.17}$ & - & $3.99_{1.24}^{0}$ & - & 4.30 & 41.58 & 41.65 & 8 \\
\hline 3C $98.0(0064600301)$ & Nucleus $^{* *}$ & MEPL & $0.72_{0.52}^{0.94}$ & $6.98_{4.45}^{9.76}$ & $0.17_{0.11}^{0.21}$ & $0.99_{0.43}^{1.61}$ & 0.72 & 42.93 & 42.67 & - \\
\hline NGC 3079 (2038) & Ring* & MEPL & $0.01_{0.00}^{0.24}$ & $0.07_{0.00}^{0.16}$ & $0.65_{0.61}^{0.69}$ & $2.16_{1.76}^{2.75}$ & 0.91 & 39.92 & 39.63 & 78 \\
\hline NGC 3079 (0110930201) & Nucleus** & MEPL & $0.59_{0.00}^{1.82}$ & - & $0.23_{0.20}^{0.28}$ & $1.24_{0.57}^{4.96}$ & 1.04 & 40.36 & 41.92 & - \\
\hline IC 2560 (4908) & Ring* & MEPL & - & $0.00_{0.00}^{2.18}$ & $0.29_{0.21}^{0.41}$ & $0.82_{0.28}^{2.92}$ & 1.56 & 39.22 & 39.62 & 11 \\
\hline IC 2560 (0203890101) & Nucleus** & 2ME2PL & - & $33.94_{26.02}^{44.73}$ & $0.09_{0.07}^{0.09}\left(0.60_{0.57}^{0.63}\right)$ & $1.32_{1.03}^{1.60}$ & 1.14 & 40.61 & 41.08 & - \\
\hline NGC 3393 (4868) & Ring* & MEPL & $0.15_{0.00}^{0.54}$ & $0.03_{0.00}^{0.39}$ & $0.19_{0.08}^{0.31}$ & $2.92_{2.32}^{3.70}$ & 1.35 & 41.02 & 39.85 & 17 \\
\hline NGC 3393 (0140950601) & Nucleus $^{* *}$ & 2ME2PL & - & $21.98_{8.83}^{64.98}$ & $0.09_{0.08}^{0.11}\left(0.59_{0.54}^{0.62}\right)$ & $1.19_{0.72}^{1.69}$ & 1.08 & 41.10 & 41.11 & - \\
\hline NGC 4507 (12292) & Ring* & ME2PL & - & $66.35_{51.97}^{82.45}$ & $0.77_{0.64}^{0.87}$ & $2.40_{1.97}^{2.77}$ & 1.40 & 40.48 & 41.44 & 13 \\
\hline NGC 4507 (0653870401) & Nucleus** & 2ME2PL & - & $37.50_{32.87}^{42.42}$ & $0.15_{0.14}^{0.17}\left(0.69_{0.64}^{0.77}\right)$ & $0.58_{0.36}^{0.78}$ & 1.06 & 41.71 & 42.66 & - \\
\hline NGC 5194 (3932) & Ring* & ME2PL & $0.11_{0.031}^{0.15}$ & $11.18_{9.18}^{13.61}$ & $0.57_{0.54}^{0.59}$ & $3.78_{3.24}^{4.00}$ & 1.34 & 40.74 & 39.69 & 91 \\
\hline NGC 5194 (0112840201) & Nucleus $^{* *}$ & 2ME2PL & $3.43_{0.06}^{10.37}$ & $106.33_{53.17}^{190.36}$ & $0.15_{0.12}^{0.19}\left(0.69_{0.63}^{0.76}\right)$ & $3.44_{3.37}^{3.51}$ & 0.92 & 41.70 & 40.75 & - \\
\hline MARK 273 (809) & Ring* & MEPL & $0.00_{0.00}^{0.11}$ & $0.00_{0.00}^{0.11}$ & $0.63_{0.54}^{0.69}$ & $2.65_{2.40}^{3.44}$ & 1.27 & 41.05 & 40.78 & 31 \\
\hline MARK 273 (0101640401) & Nucleus $^{* *}$ & 2ME2PL & - & $55.97_{40.39}^{78.04}$ & $0.26_{0.00}^{0.53}\left(0.80_{0.65}^{1.00}\right)$ & $1.39_{0.98}^{1.74}$ & 1.25 & 42.23 & 42.68 & - \\
\hline Circinus (10937) & Ring* & ME2PL & - & $123.38_{48.72}^{150.24}$ & $0.75_{0.64}^{0.83}$ & $1.81_{1.74}^{2.08}$ & 1.05 & 40.18 & 40.30 & 28 \\
\hline Circinus (0656580601) & Nucleus** & 2ME2PL & - & $39.57_{35.25}^{44.55}$ & $0.03_{0.02}^{0.03}\left(0.58_{0.50}^{0.61}\right)$ & $1.41_{1.34}^{1.49}$ & 1.68 & 40.49 & 40.72 & - \\
\hline NGC 6300 (10292) & Ring* & $\mathrm{ME}$ & $56.21_{42.23}^{83.99}$ & - & $0.70_{0.59}^{1.09}$ & - & 2.00 & 40.00 & 40.74 & 5 \\
\hline NGC 6300 (0059770101) & Nucleus** & $2 \mathrm{PL}$ & - & $32.45_{27.67}^{37.86}$ & - & $2.52_{2.07}^{2.98}$ & 0.85 & 40.84 & 40.58 & - \\
\hline NGC 7212 (4078) & Ring* & PL & $0.00_{0.00}^{0.12}$ & - & - & $2.11_{1.75}^{2.88}$ & 3.08 & 40.57 & 40.91 & 16 \\
\hline NGC 7212 (0200430201) & Nucleus $^{* *}$ & 2ME2PL & - & $121.46_{49.26}^{391.31}$ & $0.17_{0.11}^{0.22}\left(0.67_{0.58}^{0.80}\right)$ & $0.37_{0.10}^{0.66}$ & 1.25 & 41.66 & 42.72 & - \\
\hline NGC $7319(7924)$ & Ring* & ME2PL & - & $53.32_{30.39}^{93.89}$ & $0.61_{0.52}^{0.69}$ & $3.34_{2.94}^{3.78}$ & 0.68 & 42.72 & 41.93 & 17 \\
\hline NGC 7319 (0021140201) & Nucleus** & 2ME2PL & - & $49.09_{56.28}^{42.28}$ & $0.33_{0.41}^{0.16}\left(0.73_{0.84}^{0.62}\right)$ & $0.93_{1.23}^{0.61}$ & 1.23 & 41.75 & 42.78 & - \\
\hline
\end{tabular}

Notes. Column 1: name and obsID in parenthesis; Col. 2: extracted region; Col. 3: best-fit model; Cols. 4-8: parameters of the best-fit model ( ${ }^{(a)} n$ units of $10^{22} \mathrm{~cm}^{-2}$ ); Cols. 9 and 10: soft and hard intrinsic luminosities; and Col. 11: the percentage of the number counts contribution from the ring to the $r_{e x t}$ aperture Chandra data in the $0.5-10.0 \mathrm{keV}$ band. ${ }^{(*)}$ Spectral parameters of the annular region in Chandra data. ${ }^{(*)}$ Spectral parameters of the nuclear region in XMM-Newton data when the spectral parameters of the ring from Chandra data are included in the fit. 
A\&A 579, A90 (2015)

Table A.5. Simultaneous fittings taking the contribution from the annular region given in Table A.4 into account.

\begin{tabular}{|c|c|c|c|c|c|c|c|c|c|}
\hline ObsID & $\begin{array}{c}N_{\mathrm{H} 1} \\
\left(10^{22} \mathrm{~cm}^{-2}\right)\end{array}$ & $\begin{array}{c}N_{\mathrm{H} 2} \\
\left(10^{22} \mathrm{~cm}^{-2}\right)\end{array}$ & $\begin{array}{c}k T \\
(\mathrm{keV})\end{array}$ & $\Gamma$ & $\begin{array}{l}\text { Norm }_{1} \\
\left(10^{-4}\right)\end{array}$ & $\begin{array}{l}\mathrm{Norm}_{2} \\
\left(10^{-4}\right)\end{array}$ & $\chi^{2} /$ d.o.f. & $\begin{array}{c}\log \left(L_{\text {soft }}\right) \\
(0.5-2 \mathrm{keV})\end{array}$ & $\begin{array}{r}\log \left(L_{\mathrm{hard}}\right) \\
(2-10 \mathrm{keV})\end{array}$ \\
\hline (1) & (2) & (3) & (4) & (5) & (6) & (7) & (8) & (9) & (10) \\
\hline \multicolumn{10}{|c|}{ NGC 424} \\
\hline 0002942301 & - & $24.49_{18.65}^{31.94}$ & $0.09_{0.07}^{0.10}\left(0.67_{0.61}^{0.73}\right)$ & $1.82_{1.46}^{2.15}$ & $0.72_{0.58}^{0.88}$ & $6.70_{3.11}^{13.11}$ & $138.97 / 103$ & $41.74_{41.72}^{41.76}$ & $41.87_{41.81}^{41.92}$ \\
\hline 3146 & & & & & & & & $41.74_{41.72}^{41.76}$ & $41.84_{41.79}^{41.89}$ \\
\hline \multicolumn{10}{|c|}{ NGC 788} \\
\hline 0601740201 & - & $46.61 \begin{array}{r}51.35 \\
42.14\end{array}$ & $0.11_{0.09}^{0.12}\left(0.71_{0.67}^{0.75}\right)$ & $1.28_{1.06}^{1.51}$ & $0.35_{0.29}^{0.41}$ & $12.43_{7.55}^{20.51}$ & $262.36 / 205$ & $42.11_{42.04}^{42.17}$ & $42.63_{42.60}^{42.66}$ \\
\hline 11680 & & & & & & & & $42.11_{42.04}^{42.17}$ & $42.59 \underset{42.56}{42.62}$ \\
\hline \multicolumn{10}{|c|}{ MARK 573} \\
\hline 0200430701 & - & $11.40_{0.00}^{53.26}$ & $0.12_{0.11}^{0.15}\left(0.71_{0.67}^{0.76}\right)$ & $2.67_{2.32}^{2.91}$ & $0.02_{0.01}^{0.02}$ & $0.51_{0.43}^{0.59}$ & $198.75 / 171$ & $41.75_{41.74}^{41.76}$ & $41.41_{41.31}^{41.48}$ \\
\hline 7745 & & & & & & & & $41.71_{41.70}^{41.73}$ & $41.40_{41.30}^{41.49}$ \\
\hline \multicolumn{10}{|c|}{ MARK 1066} \\
\hline 0201770201 & $0.12_{0.03}^{0.24}$ & $82.29_{45.83}^{175.04}$ & $0.68_{0.62}^{0.79}$ & $2.02_{1.71}^{2.36}$ & $0.53_{0.40}^{0.71}$ & $7.11_{2.23}^{25.58}$ & $96.11 / 69$ & $41.40_{41.38}^{41.42}$ & $41.44_{41.33}^{41.53}$ \\
\hline 4075 & & & & & & & & $41.39_{41.37}^{41.41}$ & $41.42_{41.32}^{41.49}$ \\
\hline \multicolumn{10}{|c|}{ 3C 98.0} \\
\hline 0064600301 & $0.45_{0.09}^{0.61}$ & $6.14_{4.99}^{7.46}$ & $0.17_{0.12}^{0.28}$ & $0.85_{0.59}^{1.16}$ & $9.85_{0.51}^{13.56}$ & $1.25_{0.76}^{2.02}$ & $94.67 / 73$ & $42.41_{42.11}^{42.58}$ & $42.64_{42.58}^{42.69}$ \\
\hline 10234 & & & & & & & & $42.40_{42.10}^{42.58}$ & $42.59_{42.54}^{42.64}$ \\
\hline \multicolumn{10}{|c|}{ IC 2560} \\
\hline 0203890101 & - & $31.42_{25.13}^{39.74}$ & $0.09 \underset{0.07}{0.09}\left(0.60_{0.57}^{0.62}\right)$ & $1.28_{1.03}^{1.52}$ & $0.12_{0.10}^{0.15}$ & $0.67_{0.38}^{1.14}$ & $387.65 / 309$ & $40.57_{40.56}^{40.58}$ & $41.05_{41.03}^{41.08}$ \\
\hline 4908 & & & & & & & & $40.56_{40.55}^{40.57}$ & $41.01_{40.99}^{41.04}$ \\
\hline \multicolumn{10}{|c|}{ NGC 3393} \\
\hline 0140950601 & - & $32.83 \begin{array}{l}96.98 \\
18.88\end{array}$ & $0.10_{0.09}^{0.12}\left(0.58_{0.54}^{0.61}\right)$ & $2.29 \begin{array}{l}2.72 \\
1.86\end{array}$ & $0.35_{0.26}^{0.45}$ & $2.34_{1.04}^{5.61}$ & $167.03 / 141$ & $41.44 \begin{array}{l}41.48 \\
41.39\end{array}$ & $41.26_{41.19}^{41.32}$ \\
\hline 4868 & & & & & & & & $41.533_{41.47}^{41.57}$ & $42.76_{42.69}^{42.82}$ \\
\hline \multicolumn{10}{|c|}{ NGC 4507} \\
\hline 0653870401 & - & $41.19_{38.85}^{43.59}$ & $0.15_{0.14}^{0.17}\left(0.70_{0.67}^{0.76}\right)$ & $0.66_{0.53}^{0.79}$ & $0.56_{0.50}^{0.62}$ & $6.25_{4.58}^{8.45}$ & $784.58 / 659$ & $41.81_{41.80}^{41.82}$ & $42.73_{42.71}^{42.74}$ \\
\hline 12292 & & & & & & $13.35_{10.07}^{17.61}$ & & $42.07_{42.06}^{42.08}$ & $42.94_{42.92}^{42.96}$ \\
\hline \multicolumn{10}{|c|}{ NGC 5194} \\
\hline 0112840201 & $0.10_{0.04}^{0.17}$ & $197.20_{81.98}^{255.61}$ & $0.02_{0.01}^{0.02}\left(0.65_{0.62}^{0.68}\right)$ & $3.46_{3.26}^{3.60}$ & $0.16_{0.14}^{0.20}$ & $41.71_{30.51}^{114.02}$ & $322.54 / 202$ & $40.03_{40.02}^{40.03}$ & 39.593939 .52 \\
\hline 3932 & & & & & & & & $39.84_{39.92}^{39.75}$ & $39.18_{39.11}^{39.24}$ \\
\hline \multicolumn{10}{|c|}{ MARK 273} \\
\hline 0101640401 & - & $78.85_{67.72}^{90.96}$ & $0.01_{0.01}^{0.15}\left(0.88_{0.82}^{0.99}\right)$ & $1.33_{1.17}^{1.46}$ & $0.17_{0.15}^{0.19}$ & $2.86_{2.09}^{3.85}$ & $193.34 / 131$ & $41.40_{41.39}^{41.41}$ & $42.20_{42.16}^{42.24}$ \\
\hline 809 & & $38.43_{33.42}^{43.94}$ & & & & & & $41.28_{41.26}^{41.30}$ & $42.37_{42.33}^{42.41}$ \\
\hline \multicolumn{10}{|c|}{ NGC 6300} \\
\hline 0059770101 & - & $16.56_{14.86}^{18.30}$ & & $1.07_{0.82}^{1.33}$ & $0.01_{0.01}^{0.01}$ & $0.57_{0.34}^{0.94}$ & $221.55 / 172$ & $39.55_{39.41}^{39.66}$ & $40.23_{40.19}^{40.27}$ \\
\hline 10292 & & & & & $0.15_{0.11}^{0.19}$ & $37.75_{23.34}^{61.02}$ & & $41.35_{41.23}^{41.44}$ & $41.97_{41.93}^{42.00}$ \\
\hline \multicolumn{10}{|c|}{ NGC 7212} \\
\hline 0200430201 & - & $81.67_{51.53}^{124.96}$ & $0.12_{0.08}^{0.17}\left(0.59_{0.52}^{0.66}\right)$ & $0.80_{0.57}^{1.04}$ & $0.17_{0.14}^{0.21}$ & $1.43 \begin{array}{l}2.92 \\
0.62\end{array}$ & $106.34 / 77$ & $41.81_{41.76}^{41.84}$ & $42.63_{42.56}^{42.69}$ \\
\hline 4078 & & & & & & & & $41.80_{41.74}^{41.84}$ & $42.58_{42.52}^{42.64}$ \\
\hline \multicolumn{10}{|c|}{ NGC 7319} \\
\hline 0021140201 & & $45.98_{43.31}^{48.72}$ & $0.62_{0.58}^{0.65}$ & $1.68_{1.49}^{1.85}$ & $0.15_{0.13}^{0.17}$ & $10.15_{6.83}^{14.84}$ & $613.13 / 364$ & $42.23_{42.22}^{42.25}$ & $42.51_{42.49}^{42.53}$ \\
\hline 7924 & & & & & & $35.67_{24.35}^{51.54}$ & & $42.77_{42.74}^{42.80}$ & $43.02_{42.99}^{43.04}$ \\
\hline
\end{tabular}

Notes. Column 1: name and obsID in parenthesis; Cols. 2-7 parameters of the best-fit model; Col. 8: $\chi^{2} /$ d.o.f.; and Cols. 9 and 10: soft and hard intrinsic luminosities. 
L. Hernández-García et al.: X-ray variability of Seyfert 2s

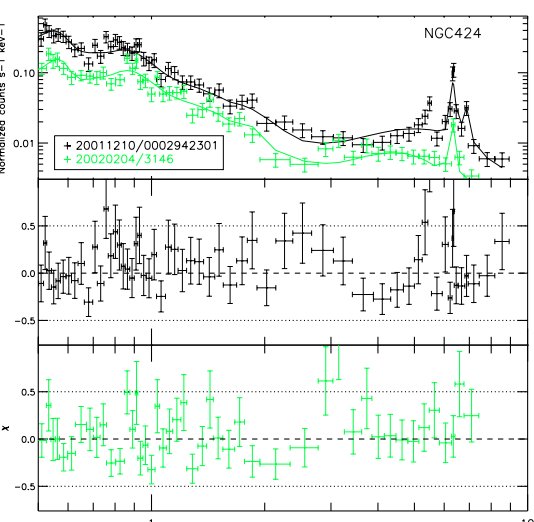

Energy (keV)

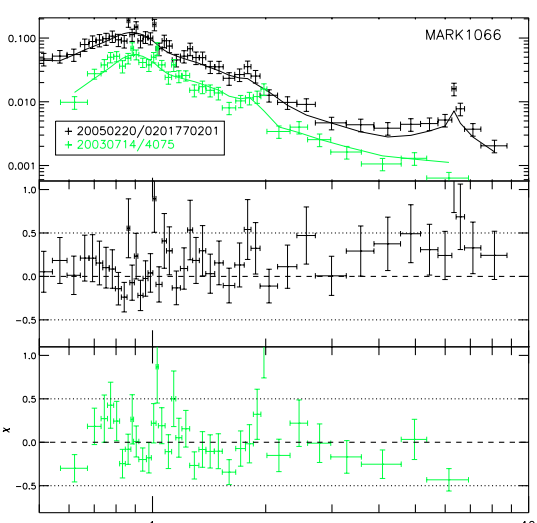

Energy (keV)
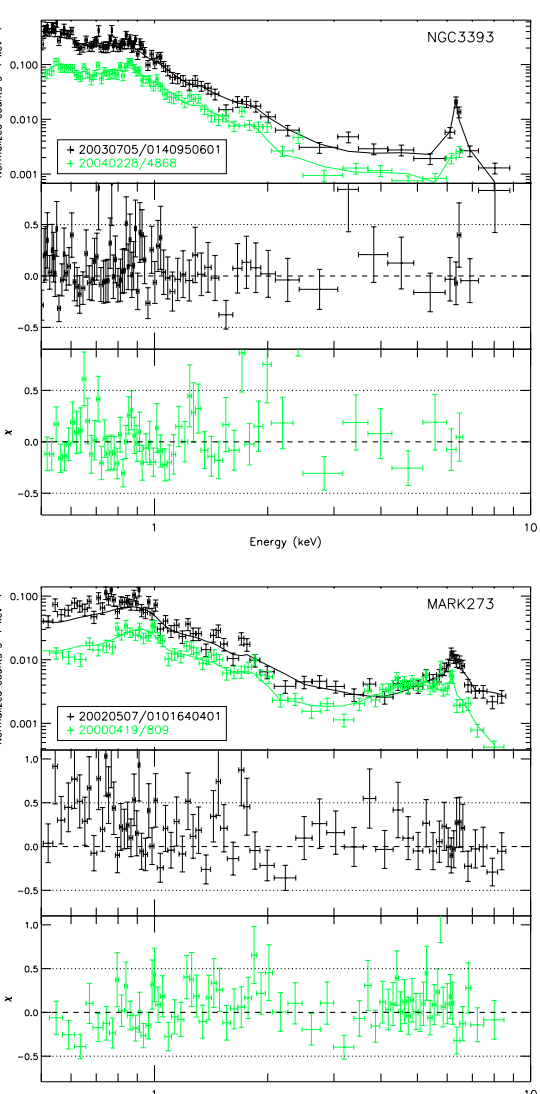

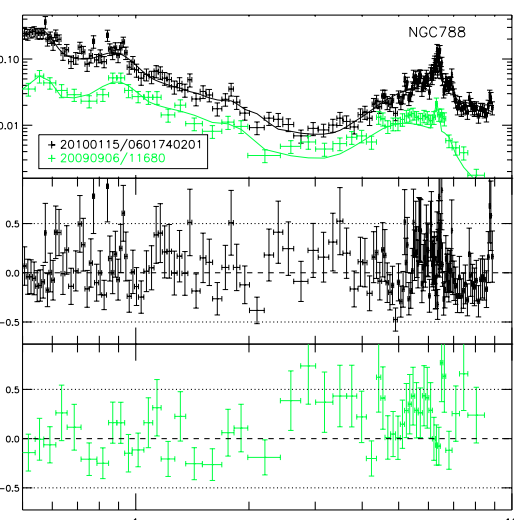

Energy (keV)

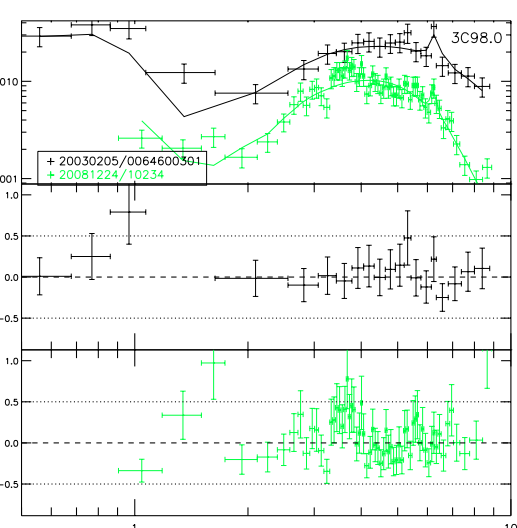

Energy (kev)
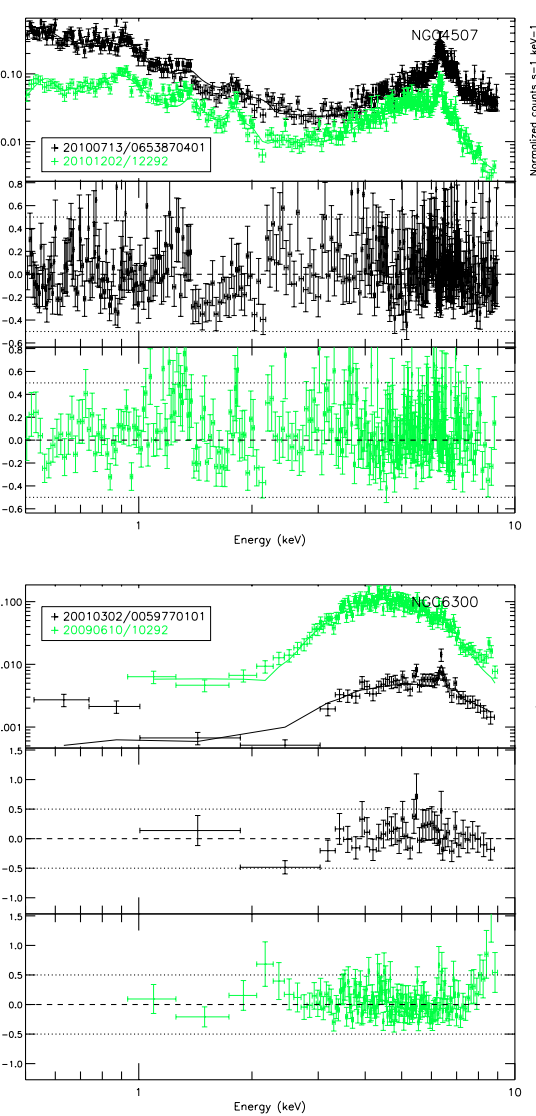
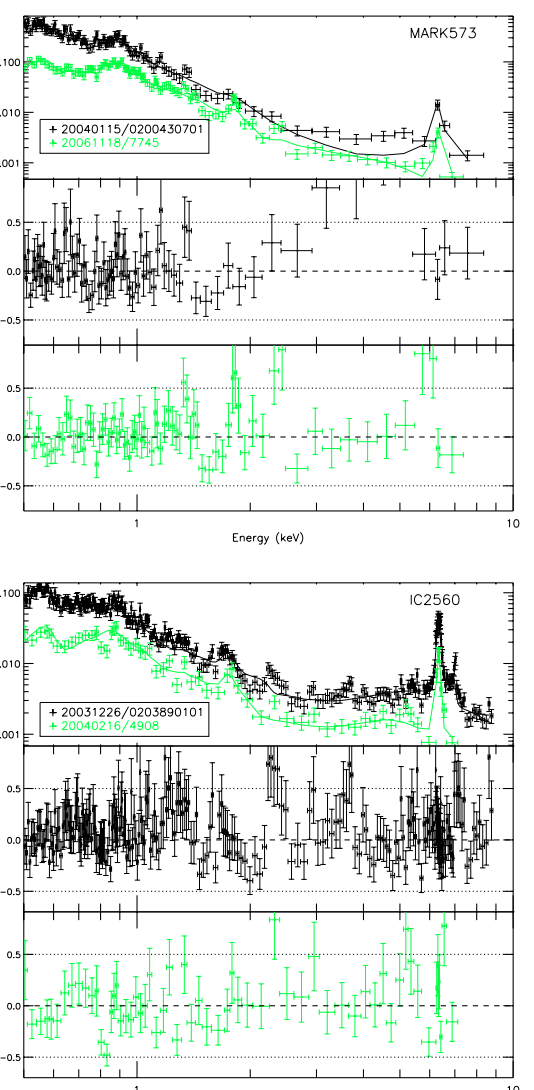

Energy (kev)

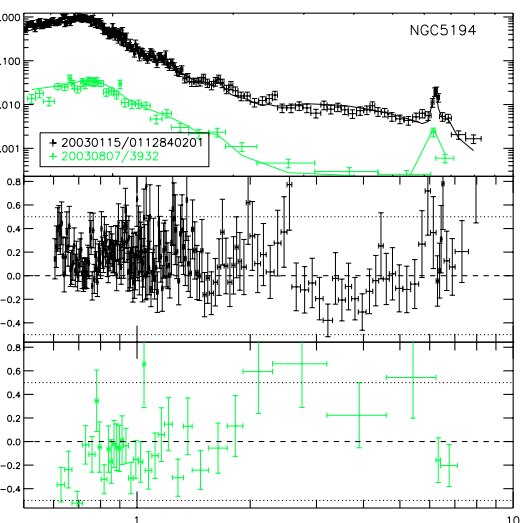

Energy (ke

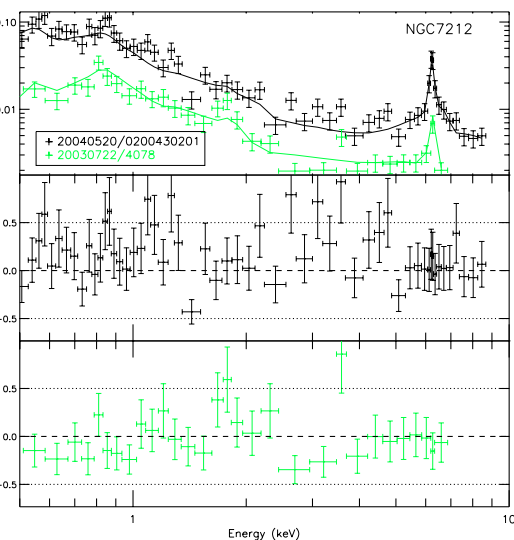

Fig. A.1. For each object, top: simultaneous fit comparing Chandra and XMM-Newton spectra; from second row on: residuals in units of $\sigma$. The legends contain the date (in the format yyyymmdd) and the obsID. The observations used for comparisons are marked with $c$ in Table A.1. 


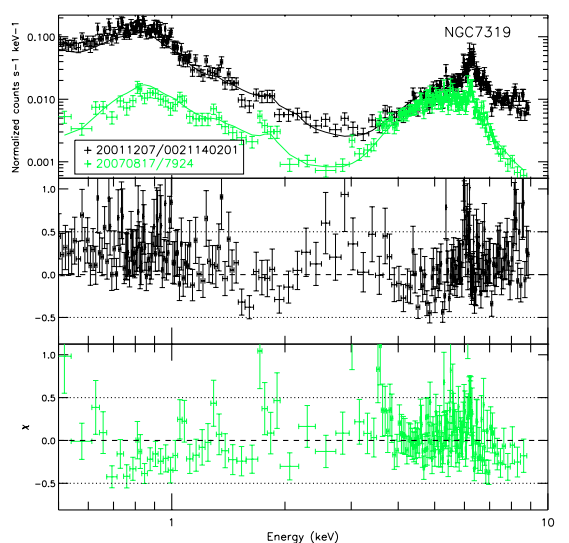

Fig. A.1. continued.
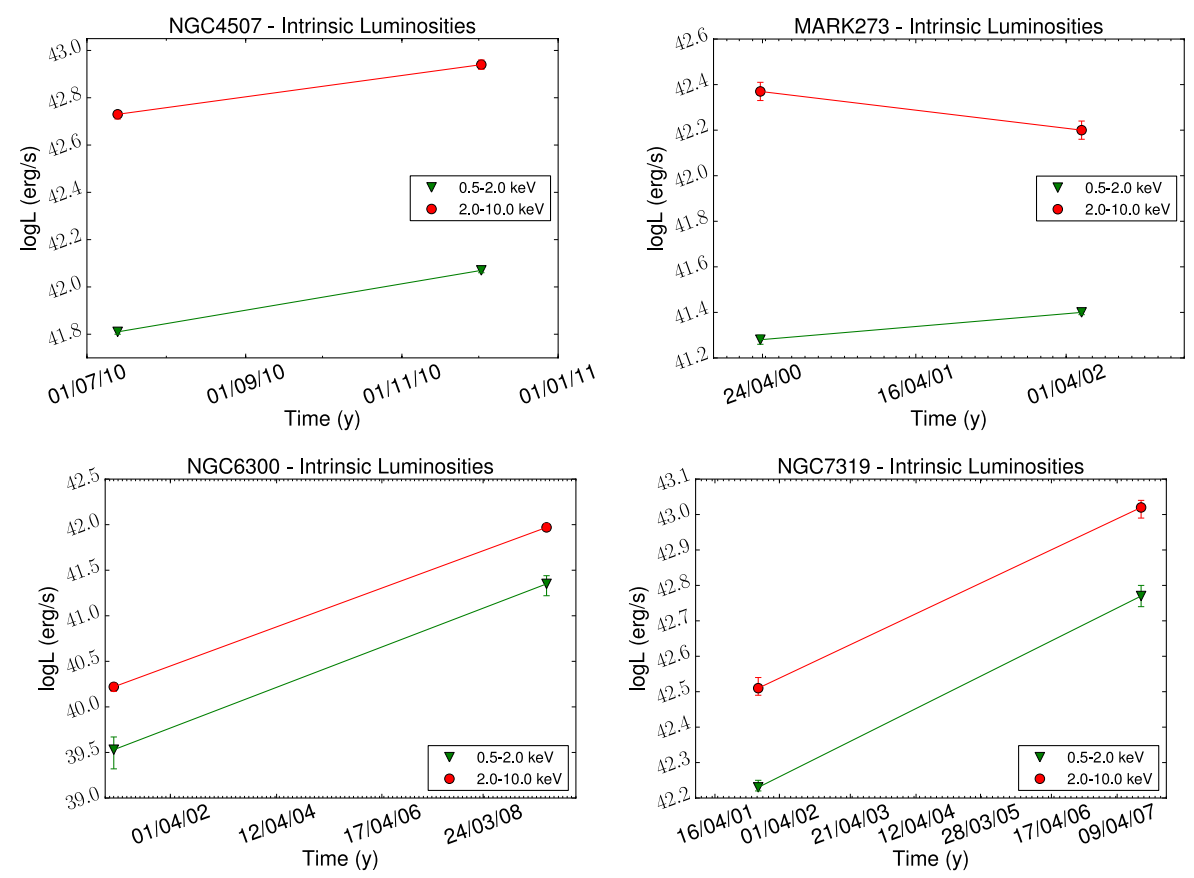

Fig. A.2. X-ray intrinsic luminosities calculated for the soft $(0.5-2.0 \mathrm{keV}$, green triangles) and hard (2.0-10.0 keV, red circles) energies in the simultaneous fitting, only for the variable objects, when Chandra and XMM-Newton data are compared.

Table A.6. Statistics of the light curves.

\begin{tabular}{lcccccc}
\hline \hline $\begin{array}{l}\text { Name } \\
(1)\end{array}$ & ObsID & Energy & $\chi^{2} /$ d.o.f. & Prob.(\%) & $\sigma_{\text {NXs }}^{2}$ & $\left\langle\sigma_{\text {NXs }}^{2}\right\rangle$ \\
\hline NGC 424 & 0550950101 & $0.5-10(1)$ & $48.7 / 40$ & 84 & $<0.0026$ & $<0.0020$ \\
& & $0.5-10(2)$ & $13.0 / 30$ & 1 & $<0.0031$ & \\
& & $0.5-2(1)$ & $38.8 / 40$ & 48 & $<0.0034$ & $<0.0027$ \\
& & $0.5-2(2)$ & $17.3 / 30$ & 3 & $<0.0041$ & \\
& & $2-10(1)$ & $33.0 / 40$ & 22 & $<0.0102$ & $<0.0077$ \\
& & $2-10(2)$ & $31.8 / 30$ & 62 & $<0.0116$ & \\
\hline
\end{tabular}

Notes. Column 1: name; Col. 2: obsID; Col. 3: energy band in keV; Cols. 4 and 5: $\chi^{2} /$ d.o.f. and the probability of being variable in the $0.5-10.0 \mathrm{keV}$ energy band of the total light curve; Col. 6: normalized excess variance, $\sigma_{\mathrm{NXS}}^{2}$; and Col. 8: the mean value of the normalized excess variance, $\left\langle\sigma_{\mathrm{NXS}}^{2}\right\rangle$, for each light curve and energy band. 
Table A.6. continued.

\begin{tabular}{|c|c|c|c|c|c|c|}
\hline $\begin{array}{l}\text { Name } \\
(1) \\
\end{array}$ & $\begin{array}{l}\text { ObsID } \\
(2) \\
\end{array}$ & $\begin{array}{c}\text { Energy } \\
(3) \\
\end{array}$ & $\begin{array}{c}\chi^{2} / \text { d.o.f. } \\
(4)\end{array}$ & $\begin{array}{c}\text { Prob. }(\%) \\
(5)\end{array}$ & $\begin{array}{c}\sigma_{\mathrm{NXS}}^{2} \\
(6)\end{array}$ & $\begin{array}{c}\left\langle\sigma_{\mathrm{NXS}}^{2}\right\rangle \\
(7) \\
\end{array}$ \\
\hline \multirow[t]{6}{*}{ MARK 573} & 7745 & $0.5-10$ & $44.4 / 38$ & 88 & $0.0041_{-}^{+} 0.0037$ & \\
\hline & & $0.5-2$ & $34.9 / 38$ & 39 & $<0.0096$ & \\
\hline & & $2-10$ & $76.0 / 38$ & 100 & $<0.0710$ & \\
\hline & 13124 & $0.5-10$ & $56.0 / 40$ & 95 & $<0.0108$ & \\
\hline & & $0.5-2$ & $46.9 / 40$ & 79 & $<0.0122$ & \\
\hline & & $2-10$ & $50.7 / 40$ & 88 & $<0.0900$ & \\
\hline \multirow[t]{3}{*}{ 3С 98.0} & 10234 & $0.5-10$ & $17.2 / 31$ & 2 & $<0.0157$ & \\
\hline & & $0.5-2$ & $32.3 / 31$ & 60 & $<0.2035$ & \\
\hline & & $2-10$ & $17.9 / 31$ & 3 & $<0.0169$ & \\
\hline \multirow[t]{6}{*}{ IC 2560} & 0203890101 & $0.5-10$ & $62.0 / 40$ & 99 & $0.0108+0.0043$ & \\
\hline & & $0.5-2$ & $44.5 / 40$ & 71 & $<0.0156$ & \\
\hline & & $2-10$ & $49.1 / 40$ & 85 & $<0.0268$ & \\
\hline & 4908 & $0.5-10$ & $48.1 / 40$ & 82 & $<0.0204$ & \\
\hline & & $0.5-2$ & $60.7 / 40$ & 99 & $0.0172_{-}^{+} 0.0140$ & \\
\hline & & $2-10$ & $29.3 / 40$ & 11 & $<0.0581$ & \\
\hline \multirow[t]{3}{*}{ NGC 3393} & 12290 & $0.5-10$ & $30.2 / 40$ & 13 & $<0.0109$ & \\
\hline & & $0.5-2$ & $31.3 / 40$ & 16 & $<0.0127$ & \\
\hline & & $2-10$ & $42.2 / 40$ & 62 & $<0.0724$ & \\
\hline \multirow[t]{6}{*}{ NGC 4507} & 0006220201 & $0.5-10$ & $35.4 / 30$ & 77 & $<0.0007$ & \\
\hline & & $0.5-2$ & $25.7 / 30$ & 31 & $<0.0031$ & \\
\hline & & $2-10$ & $36.4 / 30$ & 81 & $<0.0009$ & \\
\hline & 12292 & $0.5-10$ & $39.2 / 39$ & 54 & $<0.0026$ & \\
\hline & & $0.5-2$ & $28.9 / 39$ & 12 & $<0.0079$ & \\
\hline & & $2-10$ & $47.6 / 39$ & 84 & $<0.0039$ & \\
\hline \multirow[t]{22}{*}{ NGC 5194} & 3932 & $0.5-10$ & $50.7 / 40$ & 88 & $<0.0311$ & \\
\hline & & $0.5-2$ & $50.0 / 40$ & 87 & $<0.0364$ & \\
\hline & & $2-10$ & $42.0 / 40$ & 62 & $<0.2008$ & \\
\hline & 13813 & $0.5-10(1)$ & $58.7 / 40$ & 97 & $<0.0568$ & $0.0209_{-}^{+} 0.0190$ \\
\hline & & $0.5-10(2)$ & $36.2 / 40$ & 46 & $<0.0379$ & \\
\hline & & $0.5-10(3)$ & $32.8 / 40$ & 22 & $<0.0366$ & \\
\hline & & $0.5-10(4)$ & $58.8 / 40$ & 97 & $0.0335_{-}^{+} 0.0185$ & \\
\hline & & $0.5-2(1)$ & $84.8 / 40$ & 100 & $0.0572_{-}^{+} 0.0330$ & $0.0373_{-}^{+} 0.0289$ \\
\hline & & $0.5-2(2)$ & $36.5 / 40$ & 37 & $<0.0454$ & \\
\hline & & $0.5-2(3)$ & $31.8 / 40$ & 18 & $<0.0435$ & \\
\hline & & $0.5-2(4)$ & $60.0 / 40$ & 98 & $0.0236_{-}^{+} 0.0217$ & \\
\hline & & $2-10(1)$ & $28.1 / 40$ & 18 & $<0.2318$ & $<0.1218$ \\
\hline & & $2-10(2)$ & $33.8 / 40$ & 26 & $<0.2203$ & \\
\hline & & $2-10(3)$ & $30.6 / 40$ & 14 & $<0.2473$ & \\
\hline & & $2-10(4)$ & $28.9 / 40$ & 10 & $<0.2716$ & \\
\hline & 13812 & $0.5-10(1)$ & $48.9 / 40$ & 84 & $<0.0401$ & $<0.0227$ \\
\hline & & $0.5-10(2)$ & $37.9 / 40$ & 43 & $<0.0382$ & \\
\hline & & $0.5-10(3)$ & $44.6 / 40$ & 72 & $<0.0398$ & \\
\hline & & $0.5-2(1)$ & $44.8 / 40$ & 72 & $<0.0485$ & $<0.0273$ \\
\hline & & $0.5-2(2)$ & $40.9 / 40$ & 57 & $<0.0459$ & \\
\hline & & $0.5-2(3)$ & $40.5 / 40$ & 55 & $<0.0474$ & \\
\hline & & $2-10(1)$ & $38.1 / 40$ & 45 & $<0.2280$ & $<0.1423$ \\
\hline
\end{tabular}


Table A.6. continued.

\begin{tabular}{|c|c|c|c|c|c|c|}
\hline $\begin{array}{l}\text { Name } \\
(1)\end{array}$ & $\begin{array}{c}\text { ObsID } \\
(2) \\
\end{array}$ & $\begin{array}{c}\text { Energy } \\
(3) \\
\end{array}$ & $\begin{array}{c}\chi^{2} / \text { d.o.f. } \\
(4)\end{array}$ & $\begin{array}{c}\text { Prob. }(\%) \\
(5)\end{array}$ & $\begin{array}{c}\sigma_{\mathrm{NXS}}^{2} \\
(6) \\
\end{array}$ & $\begin{array}{c}\left\langle\sigma_{\mathrm{NXS}}^{2}\right\rangle \\
(7)\end{array}$ \\
\hline & & $2-10(2)$ & $26.9 / 40$ & 6 & $<0.2355$ & \\
\hline & & $2-10(3)$ & $24.1 / 40$ & 2 & $<0.2737$ & \\
\hline & 13814 & $0.5-10(1)$ & $54.5 / 40$ & 94 & $<0.0440$ & $<0.0208$ \\
\hline & & $0.5-10(2)$ & $36.9 / 40$ & 39 & $<0.0400$ & \\
\hline & & $0.5-10(3)$ & $31.6 / 40$ & 17 & $<0.0403$ & \\
\hline & & $0.5-10(4)$ & $60.1 / 40$ & 98 & $<0.0422$ & \\
\hline & & $0.5-2(1)$ & $44.5 / 40$ & 71 & $<0.0525$ & $0.0196_{-}^{+} 0.0170$ \\
\hline & & $0.5-2(2)$ & $49.0 / 40$ & 84 & $<0.0497$ & \\
\hline & & $0.5-2(3)$ & $32.7 / 40$ & 21 & $<0.0491$ & \\
\hline & & $0.5-2(4)$ & $70.3 / 40$ & 100 & $0.0310_{-}^{+} 0.0222$ & \\
\hline & & $2-10(1)$ & $23.9 / 40$ & 2 & $<0.3524$ & $<0.1471$ \\
\hline & & $2-10(2)$ & $22.7 / 40$ & 1 & $<0.2235$ & \\
\hline & & $2-10(3)$ & $28.4 / 40$ & 8 & $<0.2553$ & \\
\hline & & $2-10(4)$ & $24.8 / 40$ & 3 & $<0.3271$ & \\
\hline & 13815 & $0.5-10$ & $27.9 / 40$ & 7 & $<0.0351$ & \\
\hline & & $0.5-2$ & $25.1 / 40$ & 3 & $<0.0418$ & \\
\hline & & $2-10$ & $19.4 / 40$ & 1 & $<0.2777$ & \\
\hline & 13816 & $0.5-10$ & $40.1 / 40$ & 53 & $<0.0391$ & \\
\hline & & $0.5-2$ & $40.5 / 40$ & 55 & $<0.0463$ & \\
\hline & & $2-10$ & $28.9 / 40$ & 10 & $<0.2729$ & \\
\hline \multirow[t]{3}{*}{ MARK 273} & 809 & $0.5-10$ & $71.8 / 40$ & 100 & $<0.0155$ & \\
\hline & & $0.5-2$ & $49.1 / 40$ & 85 & $<0.0287$ & \\
\hline & & $2-10$ & $60.2 / 40$ & 98 & $<0.0337$ & \\
\hline \multirow[t]{3}{*}{ Circinus } & 9140 & $0.5-10$ & $48.0 / 40$ & 82 & $<0.0019$ & \\
\hline & & $0.5-2$ & $32.6 / 40$ & 21 & $<0.0075$ & \\
\hline & & $2-10$ & $45.5 / 40$ & 74 & $<0.0025$ & \\
\hline \multirow[t]{9}{*}{ NGC 7319} & 7924 & $0.5-10(1)$ & $30.3 / 40$ & 13 & $<0.0135$ & $<0.0093$ \\
\hline & & $0.5-10(2)$ & $23.9 / 40$ & 2 & $<0.0127$ & \\
\hline & & $0.5-2(1)$ & $48.7 / 40$ & 84 & $<0.0644$ & $<0.0451$ \\
\hline & & $0.5-2(2)$ & $69.6 / 40$ & 99 & $<0.0632$ & \\
\hline & & $2-10$ (1) & $37.2 / 40$ & 40 & $<0.0170$ & $<0.0116$ \\
\hline & & $2-10(2)$ & $29.5 / 40$ & 11 & $<0.0158$ & \\
\hline & 0021140201 & $0.5-10$ & $22.8 / 31$ & 16 & $<0.0051$ & \\
\hline & & $0.5-2$ & $24.1 / 31$ & 19 & $<0.0121$ & \\
\hline & & $2-10$ & $17.3 / 31$ & 2 & $<0.0089$ & \\
\hline
\end{tabular}


Table A.7. Classification of Compton-thick objects.

\begin{tabular}{|c|c|c|c|c|c|c|c|c|}
\hline Name & ObsID & (3) & $\begin{array}{c}E W \\
(\mathrm{keV}) \\
(4)\end{array}$ & $F_{\mathrm{x}} / F_{[\mathrm{OIII}]}$ & $\begin{array}{l}\text { Ref. }^{1} \\
\text { [O III] } \\
(6)\end{array}$ & CT? & Classification & $\Gamma_{\text {hard }}$ \\
\hline \multirow[t]{2}{*}{ MARK 348} & 0067540201 & $1.71_{1.64}^{1.80}$ & $0.06_{0.05}^{0.07}$ & 30.00 & 1 & $x$ & Compton-thin & \\
\hline & 0701180101 & 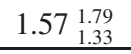 & $0.19_{0.14}^{0.25}$ & 9.06 & & $x$ & & \\
\hline \multirow[t]{3}{*}{ NGC 424} & 0002942301 & $1.03_{0.14}^{1.90}$ & $0.99_{0.74}^{1.24}$ & 1.84 & 2 & $\checkmark$ & Compton-thick & $0.54_{0.09}^{1.65}$ \\
\hline & 0550950101 & $0.16_{0.00}^{0.37}$ & $0.87_{0.82}^{0.93}$ & 1.81 & & $\checkmark$ & & \\
\hline & 3146 & $0.00_{0.00}^{1.84}$ & $0.55_{0.32}^{0.77}$ & 1.46 & & $\checkmark$ & & \\
\hline \multirow[t]{2}{*}{ MARK 573} & 7745 & $0.18_{0.00}^{2.71}$ & $2.17_{1.52}^{2.81}$ & 0.49 & 3 & $\checkmark$ & Compton-thick & $0.50_{0.00}^{3.23}$ \\
\hline & 13124 & $0.88_{0.66}^{2.08}$ & $2.05_{1.49}^{2.55}$ & 0.41 & & $\checkmark$ & & \\
\hline \multirow[t]{2}{*}{ NGC 788} & 0601740201 & $1.59_{0.97}^{2.01}$ & $0.43_{0.35}^{0.49}$ & 341.75 & 2 & $x$ & Compton-thin & \\
\hline & 11680 & $1.07_{0.17}^{2.61}$ & $0.15_{0.07}^{0.22}$ & 284.26 & & $x$ & & \\
\hline \multirow[t]{2}{*}{ ESO 417-G06 } & 0602560201 & $1.66_{1.27}^{2.00}$ & $0.18_{0.06}^{0.30}$ & 268.01 & 4 & $x$ & Compton-thin & \\
\hline & 0602560301 & $1.73_{1.31}^{2.17}$ & $0.37_{0.22}^{0.53}$ & 268.01 & & $x$ & & \\
\hline MARK 1066 & 0201770201 & $0.46_{0.00}^{1.97}$ & $0.60_{0.30}^{0.89}$ & 0.37 & 3 & $\checkmark$ & Compton-thick & $0.31_{0.00}^{0.76}$ \\
\hline \multirow[t]{3}{*}{ 3C98.0 } & 0064600101 & $1.31_{0.97}^{1.67}$ & $<0.07$ & 10.0 & 5 & $x$ & Compton-thin & \\
\hline & 0064600301 & $1.41_{0.22}^{2.13}$ & $<0.38$ & 5.89 & & $x$ & & \\
\hline & 10234 & $\begin{array}{l}0.67_{0.09}^{1.30} \\
\end{array}$ & $0.16_{0.06}^{0.27}$ & 5.01 & & $x$ & & \\
\hline \multirow[t]{7}{*}{ MARK3 } & 0111220201 & $0.05_{0.00}^{0.19}$ & $0.55_{0.52}^{0.58}$ & 0.33 & 1 & $\checkmark$ & Compton-thick & $0.42_{0.23}^{0.62}$ \\
\hline & 0009220601 & $0.36_{0.00}^{0.87}$ & $0.67_{0.56}^{0.79}$ & 0.24 & & $\checkmark$ & & \\
\hline & 0009220701 & $0.03_{0.00}^{0.53}$ & $0.60_{0.48}^{0.73}$ & 0.21 & & $\checkmark$ & & \\
\hline & 0009220901 & $0.02 \begin{array}{l}1.05 \\
0.00\end{array}$ & $0.49_{0.27}^{0.70}$ & 0.15 & & $\checkmark$ & & \\
\hline & 0009220401 & $0.01_{0.00}^{0.43}$ & $0.79_{0.63}^{0.96}$ & 0.24 & & $\checkmark$ & & \\
\hline & 0009220501 & $0.03_{0.00}^{0.48}$ & $0.63_{0.52}^{0.74}$ & 0.20 & & $\checkmark$ & & \\
\hline & 0009221601 & $0.01_{0.00}^{0.98}$ & $1.16_{0.85}^{1.46}$ & 0.24 & & $\checkmark$ & & \\
\hline \multirow[t]{5}{*}{ MARK 1210} & 4875 & $1.31_{0.74}^{1.95}$ & $0.13_{0.05}^{0.20}$ & 2.97 & 1 & $x$ & Compton-thin & \\
\hline & 9264 & $0.89_{0.40}^{1.42}$ & $0.06_{0.01}^{0.12}$ & 4.49 & & $x$ & & \\
\hline & 9265 & $1.41_{0.82}^{2.06}$ & $0.12_{0.05}^{0.19}$ & 6.34 & & $x$ & & \\
\hline & 9266 & $2.03_{1.46}^{2.86}$ & $0.10_{0.03}^{0.18}$ & 2.41 & & $x$ & & \\
\hline & 9268 & $1.24_{0.51}^{2.07}$ & $0.16_{0.061}^{0.25}$ & 3.57 & & $x$ & & \\
\hline NGC 3079 & 0110930201 & $1.58_{0.07}^{2.47}$ & $<0.26$ & 0.31 & 1 & $x$ & Compton-thin & \\
\hline \multirow[t]{2}{*}{ IC 2560} & 0203890101 & $0.22_{0.00}^{0.60}$ & $1.95_{1.79}^{2.09}$ & 7.30 & 2 & $\checkmark$ & Compton-thick & $0.69_{0.30}^{1.04}$ \\
\hline & 4908 & $<0.77$ & $1.27_{1.04}^{1.49}$ & 4.94 & & $\checkmark$ & & \\
\hline \multirow[t]{2}{*}{ NGC 3393} & 12290 & $0.55_{0.00}^{3.35}$ & $1.85_{1.45}^{2.29}$ & 0.22 & 1 & $\checkmark$ & Compton-thick & $0.42_{1.76}^{0.00}$ \\
\hline & 0140950601 & $0.95_{0.14}^{1.69}$ & $1.41_{1.00}^{1.78}$ & 0.18 & & $\checkmark$ & & \\
\hline \multirow[t]{7}{*}{ NGC 4507} & 0006220201 & $1.73_{1.59}^{1.86}$ & $0.20_{0.19}^{0.22}$ & 33.08 & 1 & $x$ & Compton-thin & \\
\hline & 0653870201 & $1.44_{1.13}^{1.72}$ & $0.44_{0.39}^{0.47}$ & 16.58 & & $x$ & & \\
\hline & 0653870301 & $1.34_{1.00}^{1.65}$ & $0.38_{0.34}^{0.42}$ & 18.60 & & $x$ & & \\
\hline & 0653870401 & $0.91_{0.44}^{1.14}$ & $0.46_{0.42}^{0.50}$ & 16.20 & & $x$ & & \\
\hline & 0653870501 & $1.01_{0.64}^{1.34}$ & $0.46_{0.41}^{0.50}$ & 17.36 & & $x$ & & \\
\hline & 0653870601 & $0.91_{0.00}^{2.16}$ & $0.43_{0.28}^{0.59}$ & 10.22 & & $x$ & & \\
\hline & 12292 & $0.87_{0.54}^{1.21}$ & $0.36_{0.32}^{0.40}$ & 24.52 & & $x$ & & \\
\hline
\end{tabular}

Notes. Column 1: name; Col. 2: obsID; Cols. 3 and 4: index of the power law and the equivalent width of the FeK $\alpha$ line from the spectral fit (PL model) in the 3-10 keV energy band; Col. 5: ratio between the individual hard X-ray luminosity (from Table A.3) and the extinction-corrected [O III] fluxes; Col. 6: references for the measure of $F_{[\mathrm{OIII}]}$; Col. 7: classification from the individual observation; Col. 8: classification of the object; and Col. 9: slope of the power law at hard energies for Compton-thick candidates from the simultaneous analysis (see Sect. 4.5).

References. (1) Bassani et al. (1999); (2) Gu et al. (2006); (3) Bian \& Gu (2007); (4) Kraemer et al. (2011); (5) Noguchi et al. (2009); (6) Panessa \& Bassani (2002); and (7) Koski (1978). 
Table A.7. continued.

\begin{tabular}{|c|c|c|c|c|c|c|c|c|}
\hline Name & ObsID & (3) & $\begin{array}{c}E W \\
(\mathrm{keV}) \\
(4)\end{array}$ & $F_{\mathrm{x}} / F_{[\mathrm{OIII}]}$ & $\begin{array}{c}\text { Ref. }^{1} \\
\text { [O III] } \\
(6)\end{array}$ & CT? & Classification & $\begin{array}{c}\Gamma_{\text {hard }} \\
\text { (9) }\end{array}$ \\
\hline NGC 4698 & 0651360401 & $0.91_{0.49}^{1.50}$ & $<0.46$ & 9.23 & 6 & $x$ & Compton-thin & \\
\hline \multirow[t]{4}{*}{ NGC 5194} & 13812 & $0.04_{0.00}^{2.21}$ & $2.75_{2.27}^{3.26}$ & 1.47 & 1 & $\checkmark$ & Compton-thick & $0.57_{0.00}^{1.74}$ \\
\hline & 13813 & $0.02_{0.00}^{2.41}$ & $4.16_{3.43}^{4.88}$ & 0.13 & & $\checkmark$ & & \\
\hline & 13814 & $0.12_{0.00}^{3.25}$ & $4.41_{3.73}^{5.14}$ & 0.17 & & $\checkmark$ & & \\
\hline & 0112840201 & $2.16_{1.29}^{3.19}$ & $0.99_{0.75}^{1.23}$ & 0.27 & & $\checkmark$ & & \\
\hline \multirow[t]{2}{*}{ MARK 268} & 0554500701 & $1.80_{1.11}^{3.43}$ & $<0.17$ & 462.73 & 7 & $x$ & Compton-thin & \\
\hline & 0554501101 & $1.71_{1.32}^{2.18}$ & $0.26_{0.18}^{0.33}$ & 351.01 & & $x$ & & \\
\hline \multirow[t]{2}{*}{ MARK 273} & 0101640401 & $0.01_{0.00}^{0.95}$ & $0.87_{0.65}^{1.12}$ & 2.75 & 1 & $\checkmark$ & Changing-look? & \\
\hline & 809 & $1.69_{0.78}^{2.77}$ & $0.21_{0.10}^{0.32}$ & 4.67 & & $x$ & & \\
\hline \multirow[t]{5}{*}{ Circinus } & 365 & $0.00_{0.00}^{0.28}$ & $2.38_{2.11}^{2.65}$ & 0.39 & 1 & $\checkmark$ & Compton-thick & $0.07_{0.00}^{0.17}$ \\
\hline & 9140 & $0.12_{0.00}^{0.33}$ & $1.90_{1.83}^{1.97}$ & 0.31 & & $\checkmark$ & & \\
\hline & 10937 & $0.00_{0.00}^{0.10}$ & $1.73_{1.63}^{1.84}$ & 0.39 & & $\checkmark$ & & \\
\hline & 0111240101 & $1.07 \begin{array}{l}1.13 \\
1.01\end{array}$ & $1.54_{1.51}^{1.56}$ & 0.35 & & $\checkmark$ & & \\
\hline & 0656580601 & $0.49_{0.41}^{0.60}$ & $1.50_{1.47}^{1.54}$ & 0.46 & & $\checkmark$ & & \\
\hline \multirow[t]{2}{*}{ NGC5 643} & 0601420101 & $0.04_{0.00}^{0.61}$ & $1.37_{1.18}^{1.56}$ & 0.29 & 1 & $\checkmark$ & Compton-thick & $0.84_{0.09}^{1.48}$ \\
\hline & 0140950101 & $0.01_{0.00}^{0.71}$ & $1.37_{1.04}^{1.69}$ & 0.37 & & $\checkmark$ & & \\
\hline \multirow[t]{2}{*}{ MARK 477} & 0651100301 & $0.93_{0.36}^{1.53}$ & $0.32_{0.22}^{0.43}$ & 0.32 & 1 & $\checkmark$ & Compton-thick & $1.02_{0.59}^{1.66}$ \\
\hline & 0651100401 & $0.88_{0.30}^{1.48}$ & $0.13_{0.05}^{0.21}$ & 0.45 & & $\checkmark$ & & \\
\hline \multirow[t]{2}{*}{ IC 4518A } & 0401790901 & $1.71_{1.29}^{2.16}$ & $0.33_{0.25}^{0.42}$ & - & - & $x$ & Compton-thin & \\
\hline & 0406410101 & $1.27 \begin{array}{c}1.60 \\
0.94\end{array}$ & $0.45_{0.38}^{0.53}$ & & & $x$ & & \\
\hline \multirow[t]{2}{*}{ ESO 138-G01 } & 0405380201 & $0.92 \begin{array}{l}1.33 \\
0.52\end{array}$ & $0.90_{0.78}^{1.01}$ & 23.10 & 2 & $\checkmark$ & Compton-thick & $1.04_{0.73}^{1.38}$ \\
\hline & 0690580101 & $0.97 \begin{array}{c}1.64 \\
0.58\end{array}$ & $1.31_{1.10}^{1.48}$ & 19.67 & & $\checkmark$ & & \\
\hline \multirow[t]{3}{*}{ NGC 6300} & 10292 & $0.57_{0.23}^{0.95}$ & $<0.08$ & 361.27 & 2 & $x$ & Compton-thin & \\
\hline & 10293 & $1.17_{0.90}^{1.69}$ & $<0.08$ & 444.46 & & $x$ & & \\
\hline & 0059770101 & $1.55_{1.03}^{2.11}$ & $0.23_{0.13}^{0.34}$ & 12.24 & & $x$ & & \\
\hline \multirow[t]{3}{*}{ NGC 7172} & 0147920601 & $1.61_{1.50}^{1.73}$ & $0.12_{0.09}^{0.14}$ & 853.54 & 1 & $x$ & Compton-thin & \\
\hline & 0202860101 & $1.58 \begin{array}{l}1.67 \\
1.49\end{array}$ & $0.09_{0.07}^{0.11}$ & 834.12 & & $x$ & & \\
\hline & 0414580101 & $\begin{array}{ll}1.71 & 1.76 \\
1.66 \\
\end{array}$ & $0.08_{0.07}^{0.09}$ & 1742.72 & & $x$ & & \\
\hline \multirow[t]{2}{*}{ NGC 7212} & 0200430201 & $0.00_{0.00}^{0.26}$ & $0.79_{0.59}^{0.99}$ & 3.83 & 2 & $\checkmark$ & Compton-thick & $0.38_{0.00}^{2.19}$ \\
\hline & 4078 & $0.00_{0.00}^{2.62}$ & $1.00_{0.61}^{1.39}$ & 3.04 & & $\checkmark$ & & \\
\hline \multirow[t]{3}{*}{ NGC 7319} & 789 & $1.43_{0.84}^{2.67}$ & $0.23_{0.12}^{0.34}$ & 38.69 & 1 & $x$ & Changing-look? & \\
\hline & 7924 & $1.89_{1.52}^{2.39}$ & $0.23_{0.18}^{0.29}$ & 82.73 & & $x$ & & \\
\hline & 0021140201 & $0.23_{0.00}^{0.65}$ & $0.83_{0.73}^{0.93}$ & 22.26 & & $\checkmark$ & & \\
\hline
\end{tabular}




\section{Appendix B: Notes and comparisons with previous results for individual objects}

In this appendix we discuss the general characteristics of the galaxies in our sample at different wavelengths, as well as comparisons with previous variability studies. We recall that longterm UV variability and short-term X-ray variations were studied only for some sources (six and ten sources, see Tables 1 and A.6, respectively), so comparisons are only made in those cases. For the remaining objects, results from other authors are mentioned, when available.

\section{B.1. MARK 348}

MARK 348, also called NGC 262, is an interacting galaxy (with NGC 266, Pogge \& Eskridge 1993). It was optically classified as a Seyfert 2 (Koski 1978), while it shows broad lines in polarized light (Miller \& Goodrich 1990). It shows a spiral nuclear structure (see HST image in Appendix C.1). VLBI observations showed a compact radio core and jets structure at radio frequencies and revealed variations on timescales from months to years at 6 and $21 \mathrm{~cm}$ (Neff \& de Bruyn 1983). The XMM-Newton image shows that the soft X-ray emission is very weak in this object (see Appendix C.1), which was classified as a Compton-thin object (e.g., Awaki et al. 2006).

This galaxy was observed twice with XMM-Newton in 2002 and 2013 and once with Chandra in 2010. Recently, Marchese et al. (2014) have compared XMM-Newton and Suzaku data from 2002 and 2008. They fitted the data with a power law component transmitted through three absorbers (one neutral and two ionized), obtaining intrinsic luminosities of $\log (L(2-10 \mathrm{keV}))=43.50$ and 43.51 , respectively. They reported variations attributed to changes in the column density of the neutral and one of the ionized absorbers, together with a variation in the ionization level of the same absorber, on timescales of months. They did not report variations in $\Gamma$ and/or the continuum of the power law. Variations in the absorbing material on timescales of weeks/months were also reported by Smith et al. (2001) using RXTE data from 1996-97, but accompanied with continuum variations on timescales of $\sim 1$ day. They obtained luminosities in the range $\log (L(2-10 \mathrm{keV}))=[42.90-43.53]$. These results agreed with those later reported by Akylas et al. (2002), who analyzed the same observations plus 25 RXTE observations. Our analysis shows that variations between the two $X M M$-Newton observations are due to changes in the nuclear continuum, but variations in the absorbing material are not required. These differences may be related to the different instruments involved in the analyses.

Awaki et al. (2006) did not find short-term variations from the analysis of the XMM-Newton data from 2002.

In the 14-195 keV energy band, Soldi et al. (2014) estimated a variability amplitude of 25[22-28]\% using data from the Swift/BAT 58-month survey.

\section{B.2. NGC 424}

NGC 424 was optically classified as a Seyfert 2 galaxy (Smith 1975), and broad lines have been detected in polarized light (Moran et al. 2000). At radio frequencies, it was observed with VLA at 6 and $20 \mathrm{~cm}$, showing an extended structure (Ulvestad \& Wilson 1989). A possible mid-IR variability was reported by Hönig et al. (2012) between 2007 and 2009, but it could also be due to an "observational inaccuracy". In X-rays, it is a Comptonthick source (Baloković et al. 2014).
It was observed twice with XMM-Newton in 2008 and 2011, and once with Chandra in 2002. Matt et al. (2003) studied XMM-Newton and Chandra data from 2001 and 2002. Both spectra were fitted with a model consisting on two power laws, a cold reflection component (PEXRAV), and narrow Gaussian lines. They report the same luminosity for the two spectra, $\log (L(2-10 \mathrm{keV}))=41.68$, indicating no variations. LaMassa et al. (2011) studied the same data set. They found no differences between the spectra and therefore fitted the data simultaneously with a simpler model, the 2PL. They estimated an intrinsic luminosity of $\log (L(2-10 \mathrm{keV}))=41.56[41.39-41.75]$. With the same data set, we did not find variations and obtained similar hard X-ray luminosities (41.85[41.79-41.92]).

We did not find short-term variations from the XMM-Newton light curve from 2008.

\section{B.3. MARK 573}

MARK 573 (also called UCG 1214) is a double-barred galaxy that shows dust lanes (Martini et al. 2001, see also Appendix C.1). It was optically classified as a Seyfert 2 galaxy (Osterbrock \& Martel 1993). Observations at $6 \mathrm{~cm}$ with VLA showed a triple radio source (Ulvestad \& Wilson 1984). A pointlike source is observed in hard X-rays, while extended emission can be observed in soft X-rays, aligned with the bars (see Appendix C.1). It was classified as a Compton-thick candidate (Guainazzi et al. 2005b; Bianchi et al. 2010; Severgnini et al. 2012).

This galaxy was observed four times with Chandra between 2006 and 2010, and once with XMM-Newton in 2004. Bianchi et al. (2010) analyzed the Chandra data from 2006 and did not report flux variations when they compared their results with the analysis of Guainazzi et al. (2005b) of the XMM-Newton spectrum from 2004. Paggi et al. (2012) studied the four Chandra observations, and fitted the nuclear spectrum with a combination of a two-phased photoionized plasma plus a Compton reflection component (PEXRAV), reporting soft X-ray flux variations at $4 \sigma$ of confidence level that they attributed to intrinsic variations of the source. We did not detect variations for this source, the difference most probably because we did not use two of these observations since they are affected by a pileup fraction greater than $10 \%$.

Ramos Almeida et al. (2008) analyzed the XMM-Newton light curve and found variations of $\sim 300 \mathrm{~s}$. They argue that this is an obscured narrow-line Seyfert 1 galaxy instead of a Seyfert 2, based on near-IR data. We analyzed two Chandra light curves but variations were not found.

\section{B.4. NGC 788}

This galaxy was optically classified as a Seyfert 2 by Huchra et al. (1982). A radio counterpart was detected with VLA data (Nagar et al. 1999). In X-rays, it was classified as a Comptonthin candidate using ASCA data (de Rosa et al. 2012), and it shows a point-like source in the $4.5-8 \mathrm{keV}$ energy band (see Appendix C.1).

It was observed once with Chandra in 2009 and once with XMM-Newton in 2010. Long-term variability analyses of this source were not found in the literature. We did not find variations between the observations.

Variations in this source in the 14-195 keV energy band were studied by Soldi et al. (2014) using data from the Swift/BAT 
58-month survey. They report an amplitude of the intrinsic variability of $15[11-19] \%$.

\section{B.5. ESO 417-G06}

This galaxy was optically classified as a Seyfert 2 galaxy (Maia et al. 2003). A radio counterpart was observed with VLA data (Nagar et al. 1999). It was classified as a Compton-thin candidate (Trippe et al. 2011).

This galaxy was observed twice with XMM-Newton in 2009. Long-term variability studies were not found in the literature. We found spectral variations due to changes in the absorber at hard X-ray energies.

Trippe et al. (2011) report short-term variations of a factor of about two in the count rate in the light curves from Swift/BAT during the 22-month survey.

\section{B.6. MARK 1066}

MARK 1066 is an early-type spiral galaxy (Afanas'ev et al. 1981) showing a double nucleus (Gimeno et al. 2004). It was optically classified as a Seyfert 2 by Goodrich \& Osterbrock (1983), and broad lines were not detected in polarized light $(\mathrm{Gu}$ $\&$ Huang 2002). A radio counterpart showing a jet was found by Ulvestad \& Wilson (1989). At X-rays, extended soft emission can be observed, aligned with a nuclear spiral structure observed at optical frequencies, also aligned with the IR emission (see Appendix C.1). Levenson et al. (2001) found this to be a heavily obscured AGN, with $N_{\mathrm{H}}>10^{24} \mathrm{~cm}^{-2}$ and an equivalent width of the Fe line $\sim 3 \mathrm{keV}$ using ROSAT and ASCA data; i.e., it was classified as a Compton-thick candidate.

The galaxy was observed once with Chandra in 2003 and once with XMM-Newton in 2005. Variability studies of this object were not found in the literature. We did not find any X-ray variations either.

\section{B.7. $3 C 98.0$}

Using the optical line measurements in Costero \& Osterbrock (1977), 3C 98.0 can be optically classified as a Seyfert 2 (see an optical spectrum in Appendix C.1). A nuclear core plus jet structure was observed at radio frequencies with VLA (Leahy et al. 1997).

It was observed twice with XMM-Newton in 2002 and 2003 and once with Chandra in 2008. Isobe et al. (2005) studied the two XMM-Newton data, and fitted its spectra with a thermal plus a power-law model, reporting X-ray luminosities of $\log (L(2-10 \mathrm{keV}))=42.90$ [42.88-42.93] and 42.66[42.60-42.71], respectively, indicating flux variability. These measurements agree well with ours, where variations due to the nuclear continuum were found.

Awaki et al. (2006) studied short-term variations of the XMM-Newton observation from 2003 and calculated a normalized excess variance of $\sigma_{\text {NXS }}^{2}=36[1-62] \times 10^{-3}$. We did not find short-term variations from one Chandra light curve, where upper limits of the $\sigma_{\mathrm{NXS}}^{2}$ were calculated. filter.

We did not find any long-term UV variations in the UVW1

\section{B.8. MARK 3}

It was optically classified as a Seyfert 2 galaxy (Khachikian \& Weedman 1974, see an optical spectrum in Appendix C.1).
Broad lines have been found in polarized light (Miller \& Goodrich 1990). A high resolution image at $2 \mathrm{~cm}$ with VLA data shows a double nucleus at radio frequencies (Ulvestad \& Wilson 1984). This galaxy shows extended soft X-ray emission perpendicular to the IR emission and a point-like source at hard X-rays (see Appendix C.1). It is also a Compton-thick source (Bassani et al. 1999; Goulding et al. 2012), with a column density of $1.1 \times 10^{24} \mathrm{~cm}^{-2}$ measured with BeppoSAX (Cappi et al. 1999).

It was observed 11 times with XMM-Newton between 2000 and 2012, and once with Chandra in 2012. Bianchi et al. (2005b) report variations of the normalization of the absorbed power law when comparing the XMM-Newton from 2001 with Chandra and BeppoSAX data. Guainazzi et al. (2012) studied the X-ray variability of this nucleus during 12 years of observations with Chandra, XMM-Newton, Suzaku, and Swift satellites. Their analysis was performed in the $4-10 \mathrm{keV}$ energy band. To estimate the luminosities, they fit a pure reflection model plus Gaussian lines to the spectra individually, and report a variability dynamical range greater than $70 \%$. They also used alternative models to fit the data, variations found independently of the model used. They estimated the shortest variability timescale to be $\sim 64$ days from the measurement between two statistically inconsistent measures. From our analysis, variations due to the nuclear continuum were found, with an upper limit of the variability timescale of about five months, thus in agreement with the results presented by Guainazzi et al. (2012).

Short-term variations from XMM-Newton data were found neither by González-Martín \& Vaughan (2012) nor by Cappi et al. (2006) from light curves from 2000 and 2001, respectively.

Soldi et al. (2014) reported an amplitude of the intrinic variability of 35[26-46]\% in the 14-195 keV energy band using data from the Swift/BAT 58-month survey.

\section{B.9. MARK 1210}

This galaxy, also called the Phoenix galaxy or UGC 4203, was optically classified as a Seyfert 2 by Dessauges-Zavadsky et al. (2000). Broad lines have been observed in polarized light using spectropolarimetric data (Tran et al. 1992; Tran 1995). The HST image shows a nuclear spiral structure (see Appendix C.1). A very compact radio counterpart was found with VLA at $3.5 \mathrm{~cm}$, with no evidence of a jet structure (Falcke et al. 1998). At $\mathrm{X}$-rays, a point like source is observed in the $4.5-8.0 \mathrm{keV}$ energy band (see Appendix C.1). It was classified as a Compton-thick candidate by Bassani et al. (1999). Furthermore, Guainazzi et al. (2002) classified this galaxy as a changing-look AGN because transitions from Compton-thick (ASCA data) to Compton-thin (XMM-Newton data) were found.

MARK 1210 was observed with Chandra six times between 2004 and 2008, and once with XMM-Newton in 2001. Matt et al. (2009) used Suzaku data from 2007 to study this source (caught in the Compton-thin state), and compared with previous observations from ASCA and XMM-Newton. They fit the spectra with a power law, a Compton reflection, and a thermal (MEKAL) component, and found a change in the absorber, which was about a factor of 2 higher in Suzaku data. They obtained intrinsic X-ray luminosities of $\log (L(2-10 \mathrm{keV}))=42.87$ and 43.04 for Suzaku and XMM-Newton data. Risaliti et al. (2010) simultaneously fit the five Chandra observations from 2008 using a model consisting on a doubled temperature plus power law to account for the soft energies, an absorbed power law, and a constant cold reflection component (PEXRAV). They concluded that variations are found in both the intrinsic flux and in the absorbing column density. They reported a variability time scale of $\sim 15$ days, whereby 
they estimated the physical parameters of the absorbing material, concluding that they are typical of the broad line region (BLR). Their result agrees well with ours.

Awaki et al. (2006) studied short-term variations from the $X M M-N e w t o n$ data and found $\sigma_{\mathrm{NXS}}^{2}=5.5[0.0-11.0] \times 10^{-3}$.

Soldi et al. (2014) used data from the Swift/BAT 58month survey to account for the variability amplitude $\left(S_{v}=\right.$ $24[15-32] \%$ ) in the 14-195 keV energy band.

\section{B.10. NGC 3079}

This galaxy was optically classified as a Seyfert 2 (Ho et al. 1997, based on the spectra presented in Appendix C.1). Broad lines were not detected in polarized light (Gu \& Huang 2002). The HST image shows dust lanes (Appendix C.1). A water maser and parsec-scale jets were observed at radio frequencies with VLBI (Trotter et al. 1998). The X-ray image in the 0.6-0.9 keV energy band shows strong diffuse emission, while a point-like source is detected in the $4.5-8.0 \mathrm{keV}$ energy band (see Appendix C.1). It has been classified as a Compton-thick object with BeppoSAX data $\left(N_{\mathrm{H}}=10^{25} \mathrm{~cm}^{-2}\right.$, Comastri 2004) and evidence was also found at lower energies (Cappi et al. 2006; Akylas \& Georgantopoulos 2009; Brightman \& Nandra 2011a).

It was observed once with Chandra and once with XMM-Newton, both in 2001. We did not find variability studies of this source in the literature. We did not study its variability because the extranuclear emission in Chandra data was too high to properly compare XMM-Newton and Chandra observations.

It is worth noting that NGC 3079 is classified as a Comptonthin candidate in this work but it has been classified as a Compton-thick candidate by Cappi et al. (2006) using the same $X M M-N e w t o n$ observation. Since these data have the lowest signal-to-noise ratio, this mismatch is most probably due to a problem related to the sensitivity of the data, because we used only data from the pn detector, while they combined pn, MOS1, and MOS2 data in their study; i.e., Cappi et al. (2006) data have a higher signal-to noise. We notice that cross-calibration uncertainties between pn and MOS cameras may add systematic to statistical uncertainties that can be misinterpreted as possible intrinsic variability due to large error bars (Kirsch et al. 2004; Ishida et al. 2011; Tsujimoto et al. 2011), thus preventing us from doing a variability analysis.

\section{B.11. IC 2560}

This galaxy was optically classified as a Seyfert 2 (Fairall 1986, see an optical spectrum in Appendix C.1). In hard X-rays it shows a point-like source (see Appendix C.1). It was classified as a Compton-thick object (Baloković et al. 2014).

IC 2560 was observed once with XMM-Newton in 2003 and once with Chandra in 2004. Variability studies were not found in the literature. We do not report X-ray variations for this source, either at short or long term.

\section{B.12. NGC 3393}

NGC 3393 was optically classified as a Seyfert 2 (Diaz et al. 1988, see an optical spectrum in Appendix C.1). A radio counterpart was found using VLA data, the galaxy showing a double structure (Morganti et al. 1999). The HST image shows a nuclear spiral structure aligned with the soft X-ray emission, where the spiral structure can also be appreciated; this emission is perpendicular to the disk emission, observed at optical wavelengths and aligned with the IR emission (see Appendix C.1). A pointlike source is observed at hard X-rays (see Appendix C.1). It is a Compton-thick object observed by BeppoSAX $\left(N_{\mathrm{H}}>\right.$ $10^{25} \mathrm{~cm}^{-2}$, Comastri 2004).

This galaxy was observed once with XMM-Newton in 2003 and six times with Chandra between 2004 and 2012. Variability studies were not found in the literature. We did not find X-ray variations, whether on the short or the long term.

\section{B.13. NGC 4507}

The nucleus of this galaxy was optically classified as a Seyfert 2 (Corbett et al. 2002, see an optical spectrum in Appendix C.1). Broad lines have been detected in polarized light (Moran et al. 2000). A radio counterpart was observed with VLA data (Morganti et al. 1999). In X-rays, it shows a point-like source in the hard energy band (see Appendix C.1), and it is a Comptonthin source (Bassani et al. 1999; Braito et al. 2013).

NGC 4507 was observed six times with XMM-Newton between 2001 and 2010, and once with Chandra in 2010. Matt et al. (2004) studied Chandra and XMM-Newton data from 2001. They fit the XMM-Newton spectrum with a composite of two power laws, a Compton reflection component (PEXRAV), plus ten Gaussian lines, and the Chandra spectrum with a power law plus a Gaussian line (only in the $4-8 \mathrm{keV}$ spectral range). They found that the luminosity of the Chandra data was about twice that of XMM-Newton. Marinucci et al. (2013) studied five observations from XMM-Newton in 2010. They fit the spectra with two photoionized phases using Cloudy, a thermal component, an absorbed power law, and a reflection component. They report variations of the absorber on timescales between 1.5-4 months. Braito et al. (2013) studied XMM-Newton, Suzaku, and BeppoSAX data spanning around ten years to study the X-ray variability of the nucleus. They fit the spectra with the model that best represents the Suzaku data, composed of two power laws, a PEXRAV component, and eight Gaussian lines, and found that variations are mainly due to absorption but also due to the intensity of the continuum level. They also fit the spectra with the MYTORUS mode ${ }^{11}$ and obtained similar results, although the continuum varied less. We found variations in the absorber and the normalization of the power law, in agreement with the results by Braito et al. (2013).

We did not find short-term variations from the analysis of one XMM-Newton and another Chandra light curve.

Soldi et al. (2014) report an amplitude of the intrinsic variability of 20[16-24]\% in the 14-195 keV energy band using data from the Swift/BAT 58-month survey.

\section{B.14. NGC 4698}

This galaxy was optically classified as a Seyfert 2 (Ho et al. 1997, see their spectra in Appendix C.1). González-Martín et al. (2009b) classified it as a LINER, but Bianchi et al. (2012) reconfirmed the Seyfert 2 classification using optical observations with the NOT/ALFOSC/Gr7. A radio counterpart was found by Ho \& Ulvestad (2001) at $6 \mathrm{~cm}$ with VLA data. Georgantopoulos \& Zezas (2003) state that this is an atypical Seyfert 2 galaxy because it showed no absoption and lacks the broad line region. The Chandra image revealed point-like sources around the nucleus, which can be ultraluminous X-ray sources (ULX), the closest located at $\sim 30^{\prime \prime}$ from the nucleus. In X-rays, González-Martín et al. (2009b) classified it as an AGN

11 wWW . mytorus . com 
candidate, and Bianchi et al. (2012), based on the $\log \left(L_{\mathrm{X}} / L_{[\mathrm{O} \mathrm{III]}]}\right)$ ratio, classified it as a Compton-thick candidate.

This galaxy was observed twice with XMM-Newton in 2001 and 2010, and once with Chandra in 2010. Bianchi et al. (2012) compared the XMM-Newton spectra and did not find spectral variations, in agreement with the results reported by us.

We did not find any UV variations in the UVM2 filter.

\section{B.15. NGC 5194}

NGC 5194, also known as M 51, is interacting with NGC 5195. Optical and radio observations show extended emissions to the north and south of the nucleus, resulting from outflows generated by the nuclear activity (Ford et al. 1985). The extended emission can be observed in soft X-ray energies (top-left image in Appendix C.1). Moreover, the HST image shows a dusty nuclear spiral structure that can also be observed at IR frequencies (see Appendix C.1). This galaxy was optically classified as a Seyfert 2 (Ho et al. 1997, see their optical spectra in Appendix C.1). Broad lines were not detected in polarized light (Gu \& Huang 2002). A point-like source is detected at hard $\mathrm{X}$-ray energies (see Appendix C.1). Around the nucleus, it shows at least seven ultraluminous X-ray sources (ULX), the nearest one located at $\sim 28^{\prime \prime}$ from the nucleus (Dewangan et al. 2005). It was classified as a Compton-thick source using BeppoSAX data, with $N_{\mathrm{H}}=5.6 \times 10^{24} \mathrm{~cm}^{-2}$ (Comastri 2004; see also Terashima \& Wilson 2001; Dewangan et al. 2005; Cappi et al. 2006]).

This galaxy was observed ten times with Chandra between 2000 and 2012 and six times with XMM-Newton between 2003 and 2011. LaMassa et al. (2011) studied three Chandra observations between 2000 and 2003. They simultaneously fit these spectra with the ME2PL model, with spectral values in very good agreement with our SMF0 fitting and estimated a luminosity of $\log (L(2-10 \mathrm{keV}))=38.95[38.42,39.45]$. They did not report variability between the observations. This result agrees with ours.

Fukazawa et al. (2001) did not find any short-term variability from BeppoSAX data. We studied six Chandra light curves and did not find short-term variations either.

UV variations were not detected from the UVW2 and UVM2 filters, but variations were found in the UVW1 filter. However, since this is a Compton-thick source, variations are not expected, so it is most probable that the UV emission does not come from the nucleus. Therefore the variations might be related with, for example, circumnuclear star formation.

\section{B.16. MARK 268}

This galaxy was optically classified as a Seyfert 2 by Komossa $\&$ Schulz (1997). A radio counterpart was detected with VLA data at $6 \mathrm{~cm}$ with a weaker component $1.1 \mathrm{kpc}$ away from the nucleus (Ulvestad \& Wilson 1984). XMM-Newton data show a compact source at hard X-rays (see Appendix C.1) .

It was observed twice with XMM-Newton in 2008. Variability studies were not found in the literature. We did not find variations, but we notice that observations were obtained separated by only two days.

UV variations are not found from the UVM2 and the UVW1 filters.

\section{B.17. MARK 273}

Also called UGC 8696, this galaxy is an ultraluminous infrared galaxy with a double nucleus that was optically classified as a LINER (Veilleux et al. 1995), but later reclassified as a Seyfert 2 from better S/N data (Kim et al. 1998). Optical spectra are presented in Appendix C.1, together with an HST image which shows dust lanes. VLBA observations showed a radio counterpart (e.g., Carilli \& Taylor 2000). Extended emission to the south is observed in soft X-rays, while it shows a point-like source at hard energies (Appendix C.1). It was classified as a Comptonthick candidate (Teng et al. 2009).

It was observed once with Chandra in 2000, and five times with XMM-Newton between 2002 and 2013. Balestra et al. (2005) fit the Chandra and XMM-Newton spectra with a composite of three thermal plus an absorbed PL components and found similar spectral parameters, except in the value of the column densities (41[35-47] and 69[50-85] $\times 10^{22} \mathrm{~cm}^{-2}$, respectively). This result is compatible with ours, with $N_{\mathrm{H} 2}$ being responsible for the observed variations. In the same sense, Teng et al. (2009) studied Suzaku data from 2006 and found spectral variations when comparing with Chandra and XMM-Newton data. They attributed the changes to the covering fraction of the absorber.

We found no short-term variations from the Chandra light curve or UV variations from the UVW1 filter.

\section{B.18. Circinus}

It was optically classified as a Seyfert 2 galaxy (Oliva et al. 1994), and it shows broad lines in polarized light (Oliva et al. 1998). The HST image shows dust lanes (Appendix C.1). ATCA observations show a radio counterpart, a water maser, and large radio lobes (Elmouttie et al. 1998). Circinus is a Compton-thick source (Bassani et al. 1999), which in fact was observed by BeppoSAX $\left(N_{\mathrm{H}}=4.3 \times 10^{24} \mathrm{~cm}^{-2}\right.$, Matt et al. 1999).

This galaxy was observed eight times with Chandra between 2000 and 2010, and twice with XMM-Newton in 2001 and 2014. The most comprehensive analysis of this source has recently been done by Arévalo et al. (2014), who analyzed 26 observations from NuSTAR, Chandra, XMM-Newton, Swift, Suzaku, and BeppoSAX satellites spanning 15 years and the energy range $2-79 \mathrm{keV}$. They used different models to fit the data, based on PEXMON, MyTorus, and Torus models (in XSPEC). Since different appertures were used for the analysis, they decontaminated the extranuclear emission. They conclude that the nucleus did not show variations, in agreement with our result when comparing Chandra data. Moreover, Arévalo et al. (2014) find that extranuclear sources included in the larger apertures showed variations (an ultraluminous X-ray source and a supernova remnant), also in agreement with our results when comparing XMM-Newton data, where the extranuclear sources were included, and we found variations in both the normalizations at soft and hard energies.

We analysed one Chandra light curves, but variations were not detected.

The analysis of light curves from the Swift/BAT 58-month survey by Soldi et al. (2014) showed a small variability amplitude of $11[10-12] \%$ in the $14-195 \mathrm{keV}$ energy band.

\section{B.19. NGC 5643}

This galaxy was optically classified as a Seyfert 2 (Phillips et al. 1983, see an optical spectrum in Appendix C.1), and broad lines were not detected in polarized light ( $\mathrm{Gu} \&$ Huang 2002). The 
HST image shows a nuclear spiral structure (see Appendix C.1). VLA data show a nuclear counterpart alongside fainter features extending to the east and west at radio frequencies (Morris et al. 1985). The XMM-Newton image shows a compact source at hard $\mathrm{X}$-ray energies. This is a Compton-thick object observed with BeppoSAX $\left(N_{\mathrm{H}}>10^{25} \mathrm{~cm}^{-2}\right.$, Comastri 2004).

It was observed twice with XMM-Newton in 2003 and 2009, and once with Chandra in 2004. Matt et al. (2013) analyzed the two observations from XMM-Newton, who found that the spectra are reproduced well by reflection from warm and cold matter. The spectral parameters were consistent with the same values for the two observations. Thus, variations are not observed. These results agree well with ours, where variations are not found.

\section{B.20. MARK 477}

This object was classified as a Seyfert 2 (Veron et al. 1997), and broad lines have been detected in polarized light (Tran et al. 1992; Tran 1995). The HST image reveals a structure around the nucleus, which could be a spiral or a circumnuclear ring (see Appendix C.1). A nuclear counterpart was found at $6 \mathrm{~cm}$ using VLA data (Ulvestad \& Wilson 1984). It was classified as a Compton-thick candidate (Bassani et al. 1999).

The source was observed twice with ASCA in December 1995; variations were not found when fitting a scattered power law plus a narrow line (Levenson et al. 2001).

It was observed twice with XMM-Newton in 2010. We did not find variations between these observations.

Kinney et al. (1991) studied UV variability of this source with HST, but variations were not found. We did not find UV flux variations from the UVW1 filter.

\section{B.21. IC $4518 A$}

This galaxy was optically classified as a Seyfert 2 galaxy (Zaw et al. 2009). The 2MASS image shows two interacting galaxies (see Appendix C.1). It is a Compton-thin source (Bassani et al. 1999; de Rosa et al. 2008).

It was observed twice with XMM-Newton in 2006. Variability analyses were not found in the literature. However, comparing the luminosities obtained by de Rosa et al. (2012) and Pereira-Santaella et al. $(2011)$ of $\log (L(2-10 \mathrm{keV}))=42.60$ and 42.34 for the different spectra, their results are suggestive of flux variability. In fact, these luminosities agreed with our estimates. Our analysis shows that this variability is related with the nuclear continuum.

\section{B.22. ESO 138-G01}

Alloin et al. (1992) optically classified this galaxy as a Seyfert 2. It shows a jet-like morphology at radio frequencies (Morganti et al. 1999). The XMM-Newton image shows a compact source at hard X-ray energies (see Appendix C.1). It was classified as a Compton-thick candidate (Collinge \& Brandt 2000).

This galaxy was observed three times with XMM-Newton in 2007 and 2013. Variability analyses were not found in the literature. We did not find any X-ray variations.

\section{B.23. NGC 6300}

NGC 6300 is a barred spiral galaxy, whose Seyfert 2 classification at optical frequencies was derived from the data reported in Phillips et al. (1983). The HST image shows dust lanes (see Appendix C.1). A nuclear counterpart was found at radio frequencies, without any jet structure (Ryder et al. 1996). NGC 6300 was classified as a changing-look AGN, observed in the Compton-thick state with RXTE in 1997 and in the Compton-thin state with BeppoSAX in 1999 (Guainazzi 2002).

The galaxy was observed once with XMM-Newton in 2001 and five times with Chandra during 2009. Guainazzi (2002) found variations due to a difference in the normalization of the power law when comparing BeppoSAX and RXTE data. All the observations analyzed in this work caught the object in the thin state. Variations in the normalizations at soft and hard energies were found when comparing Chandra and XMM-Newton data.

Matsumoto et al. (2004) and Awaki et al. (2005, 2006) studied the light curve from XMM-Newton data and found rapid variations at hard energies.

Variations in the 14-195 keV energy band were analyzed by Soldi et al. (2014) using data from the Swift/BAT 58-month survey, who estimated an intrinsic variability amplitude of $17[14-20] \%$.

\section{B.24. NGC 7172}

NGC 7172 is an early type galaxy located in the HCG 90 group, which shows dust lanes (Sharples et al. 1984, see also Appendix C.1). Optically classified as a Seyfert 2 (see an optical spectrum in Appendix C.1), no broad lines have been observed in polarized light (Lumsden et al. 2001). A radio core was detected with VLA data (Unger et al. 1987). At IR frequencies, Sharples et al. (1984) found variations on timescales of about three months. The nucleus of this galaxy is not detected at UV frequencies with the OM (see Table A.1). Even if Chandra data are available for this source, they suffer from strong pileup. The XMM-Newton image shows a compact source (see Appendix C.1).

Guainazzi et al. (1998) first reported X-ray flux variations in this source using ASCA data. They found short-term variations (hours) from the analysis of a light curve from 1996 and longterm variations when comparing the flux of these data with previous data from 1995, when it was about three times brighter. Risaliti (2002) studied two BeppoSAX observations taken in October 1996 and November 1997 and fit the data with an absorbed power law, a thermal component, a cold reflection, a warm reflection, and a narrow Gaussian line. They reported very similar spectral parameters for the two spectra.

This galaxy was observed once with Chandra in 2000 and three times with XMM-Newton between 2002 and 2007. LaMassa et al. (2011) analyzed the XMM-Newton spectra by fitting the data with the ME2PL model and needed to fit the normalization of the power law independently. They report luminosities of $\log (L(2-10 \mathrm{keV}))=42.96_{-}^{+} 0.03$ (for the spectrum from 2007) and $42.61_{-}^{+} 0.03$ (for the other two spectra). These results agree well with our SMF1.

Awaki et al. (2006) analyzed the XMM-Newton light curve from 2002. They did not find significant variability when computing the normalized excess variance.

At higher energies, Beckmann et al. (2007) reported an intrinsic variability of $S_{V c}=12_{-}^{+9} \%$ within 20 days using Swift/BAT data, and using data from the Swift/BAT 58-month survey, Soldi et al. (2014) report a variability amplitude of $28[25-31] \%$, both in the $14-195 \mathrm{keV}$ energy band. 


\section{B.25. NGC 7212}

This galaxy is interacting with a companion (see the 2MASS image in Appendix C.1). It was optically classified as a Seyfert 2 galaxy (Veilleux \& Osterbrock 1987, see an optical spectrum in Appendix C.1). Broad lines were detected in polarized light (Tran et al. 1992). At radio wavelengths, a nuclear counterpart was found with the interacting galaxy (Falcke et al. 1998). A point-like source is detected at hard X-rays (see Appendix C.1). It was classified as a Compton-thick candidate (Severgnini et al. 2012).

It was observed once with Chandra in 2003 and once with XMM-Newton in 2004. Bianchi et al. (2006) report the same fluxes for the two spectra, also in agreement with our results.

\section{B.26. NGC 7319}

NGC 7319 is a spiral galaxy located in the Stephan's Quintet, a group composed by six galaxies including a core of three galaxies (Trinchieri et al. 2003). These three galaxies were also observed at radio wavelengths with VLA (Aoki et al. 1999) and later with MERLIN (Xanthopoulos et al. 2004), revealing a jet structure in NGC 7319. It has been optically confirmed as a Seyfert 2 (Rodríguez-Baras et al. 2014, see an optical spectrum in Appendix C.1). The nucleus of this galaxy is not detected at UV frequencies with the OM (see Table A.1). In X-rays, a pointlike source is observed in the $4.5-8.0 \mathrm{keV}$ energy band, and it shows extended emission at soft X-ray energies (Appendix C.1).

It was observed twice with Chandra in 2000 and 2007, and once with XMM-Newton in 2001. We did not find any variability studies in the literature. We found variations in the nuclear power of the nucleus, accompained by absorber variations at soft energies.
One Chandra and the XMM-Newton light curves were analyzed, but short-term variations were not detected.

\section{Appendix C: Images}

\section{C.1. Optical spectra, and X-ray, 2MASS and optical HST images}

In this appendix we present images at different wavelengths for each energy and the optical spectrum when available from NED. In X-rays we extracted Chandra data in four energy bands: 0.6-0.9 keV (top left), 1.6-2.0 keV (top middle), 4.5-8.0 keV (top right), and $0.5-10.0 \mathrm{keV}$ (bottom left). The CSMOOTH task included in CIAO was used to adaptatively smooth the three images in the top panels (i.e., the images in the $0.5-10.0 \mathrm{keV}$ energy band are not smoothed), using a fast Fourier transform algorithm and a minimum and maximum significance level of the signal-to-noise of 3 and 4, respectively. When data from Chandra was not available, XMM-Newton images were extracted in the same energy bands, and the ASMOOTH task was used to adaptatively smooth the images. At infrared frequencies, we retrieved an image from $2 \mathrm{MASS}$ in the $K_{\mathrm{s}}$ filter ${ }^{12}$. At optical frequencies we used images from $\mathrm{HST}^{13}$, preferably in the $F 814 W$ filter, but when it was not available we retrieved an image in the F606W filter. HST data have been processed following the sharp dividing method to show the internal structure of the galaxies (Marquez \& Moles 1996). The red squares in the bottom images represent the area covered by the HST image (presented in the bottom right panel when available). In all images the gray levels extend from twice the value of the background dispersion to the maximum value at the center of each galaxy. We used IRAF ${ }^{14}$ to estimate these values.

\footnotetext{
${ }^{12}$ http://irsa.ipac.caltech.edu/applications/2MASS/IM/ interactive.html

13 http://hla.stsci.edu/

${ }^{14}$ http://iraf.noao.edu/
} 
L. Hernández-García et al.: X-ray variability of Seyfert 2s

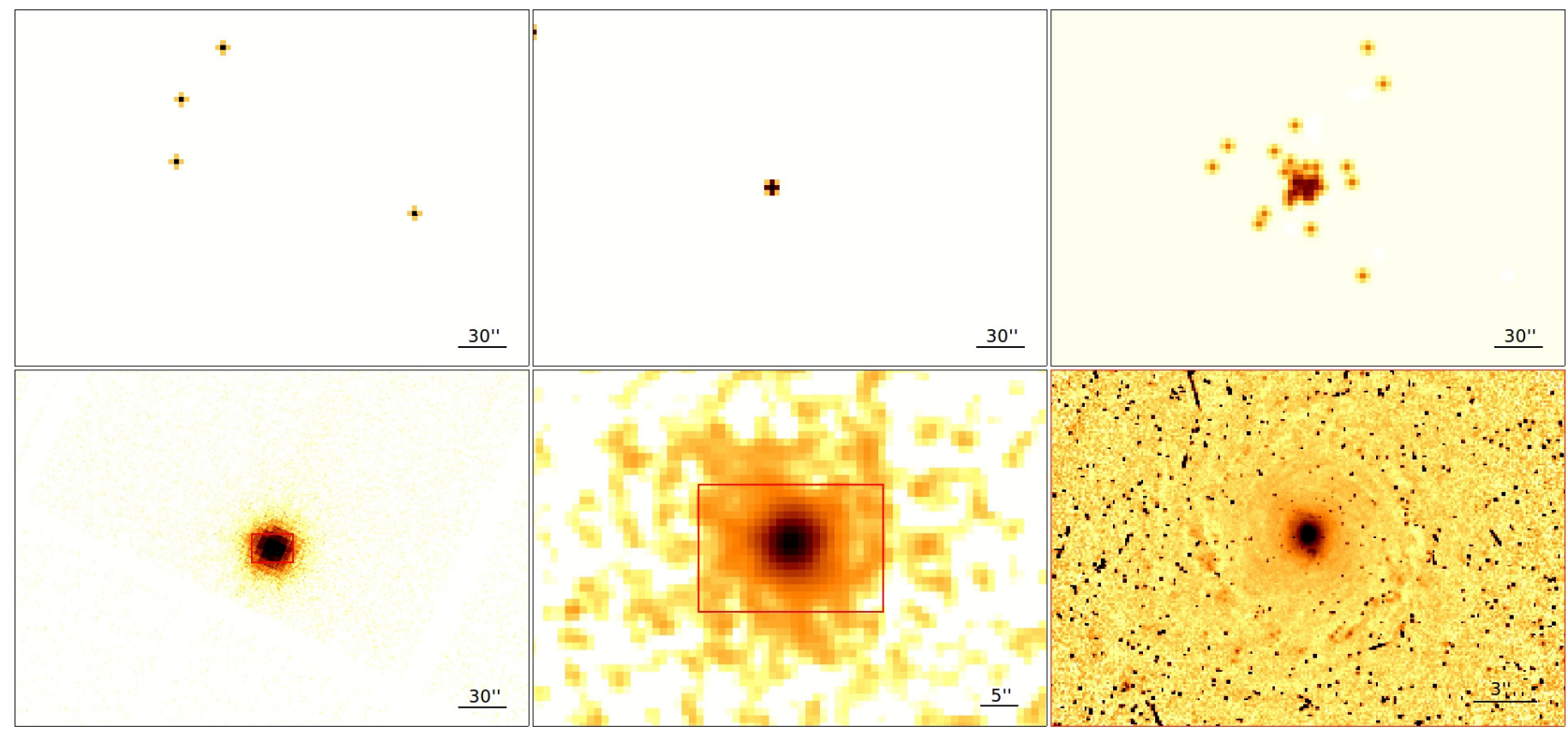

Fig. C.1. MARK 348 (XMM-Newton data). Top left: smoothed X-ray 0.6-0.9 keV energy band; top center: smoothed X-ray 1.6-2.0 keV energy band; top right: smoothed X-ray $4.5-8.0 \mathrm{keV}$ energy band; bottom left: X-ray $0.5-10.0 \mathrm{keV}$ energy band without smoothing; bottom center: 2MASS image in the $K_{\mathrm{s}}$ band; bottom right: Hubble image in the $F 606 \mathrm{~W}$ filter.

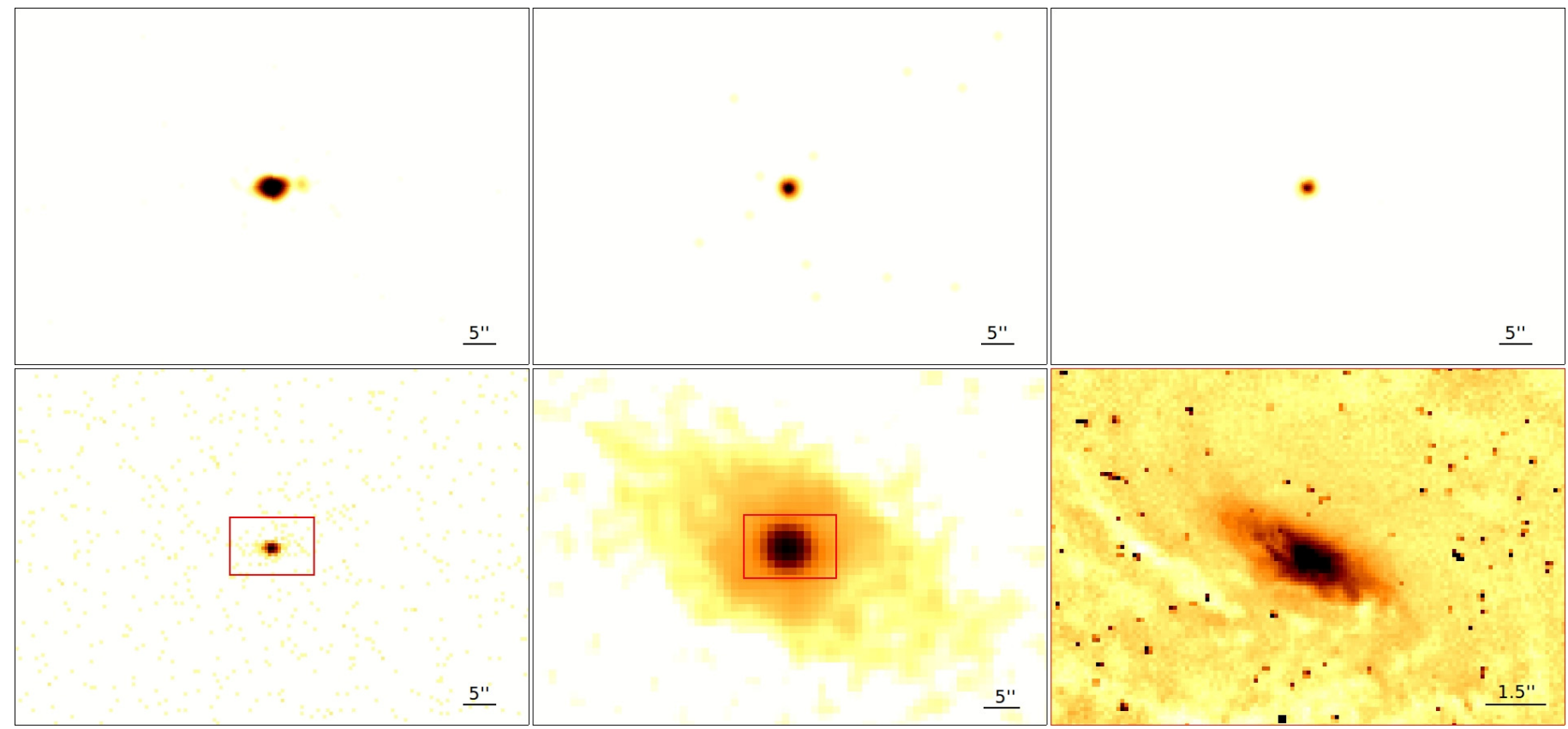

Fig. C.2. NGC 424. Top left: smoothed X-ray 0.6-0.9 keV energy band; top center: smoothed X-ray $1.6-2.0 \mathrm{keV}$ energy band; top right: smoothed X-ray $4.5-8.0 \mathrm{keV}$ energy band; bottom left: X-ray $0.5-10.0 \mathrm{keV}$ energy band without smoothing; bottom center: 2MASS image in the $K_{\mathrm{s}}$ band; bottom right: Hubble image in the $F 606 \mathrm{~W}$ filter. 


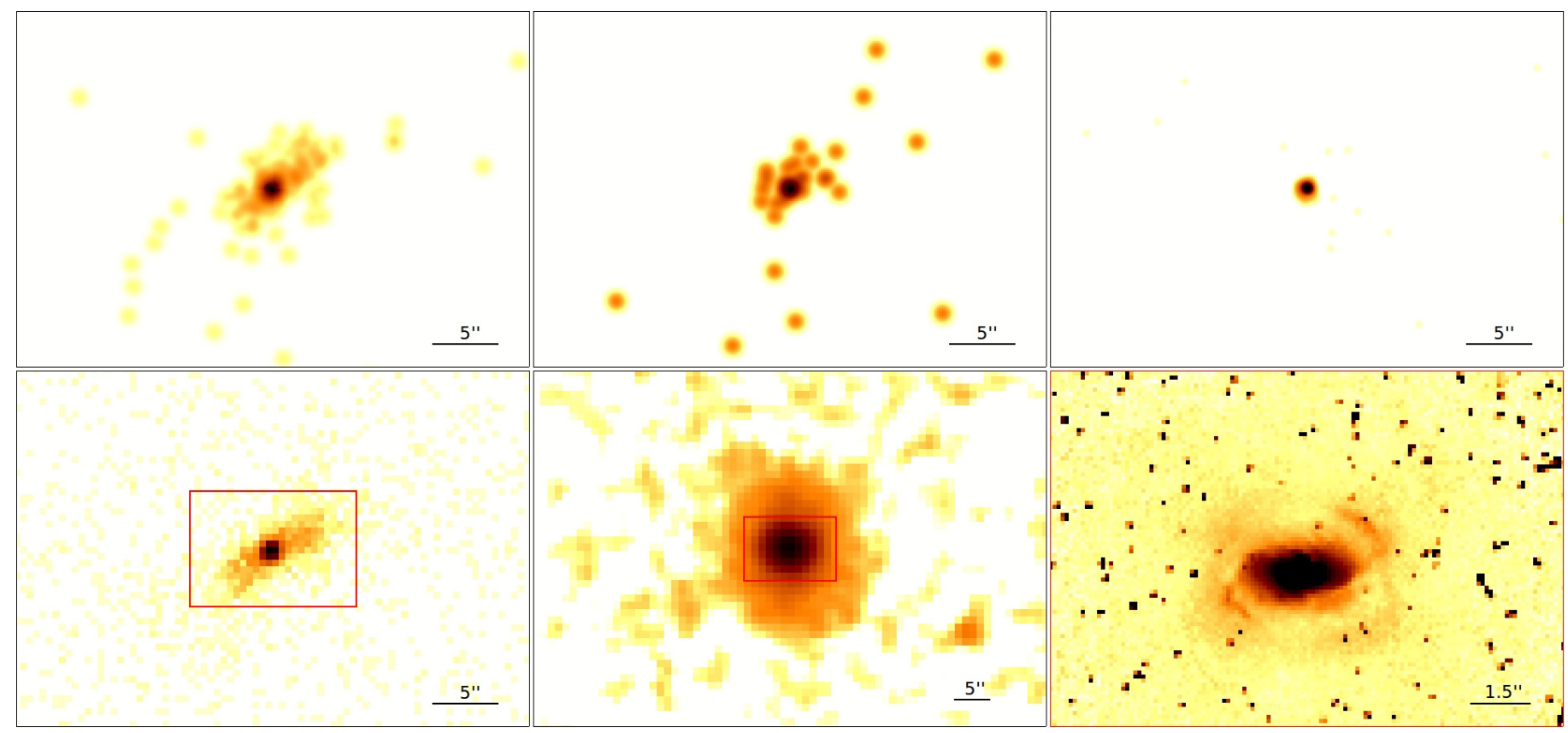

Fig. C.3. MARK 573. Top left: smoothed X-ray 0.6-0.9 keV energy band; top center: smoothed X-ray 1.6-2.0 keV energy band; top right: smoothed X-ray 4.5-8.0 keV energy band; bottom left: X-ray $0.5-10.0 \mathrm{keV}$ energy band without smoothing; bottom center: $2 \mathrm{MASS}$ image in the $K_{\mathrm{s}}$ band; bottom right: Hubble image in the $F 814 \mathrm{~W}$ filter.

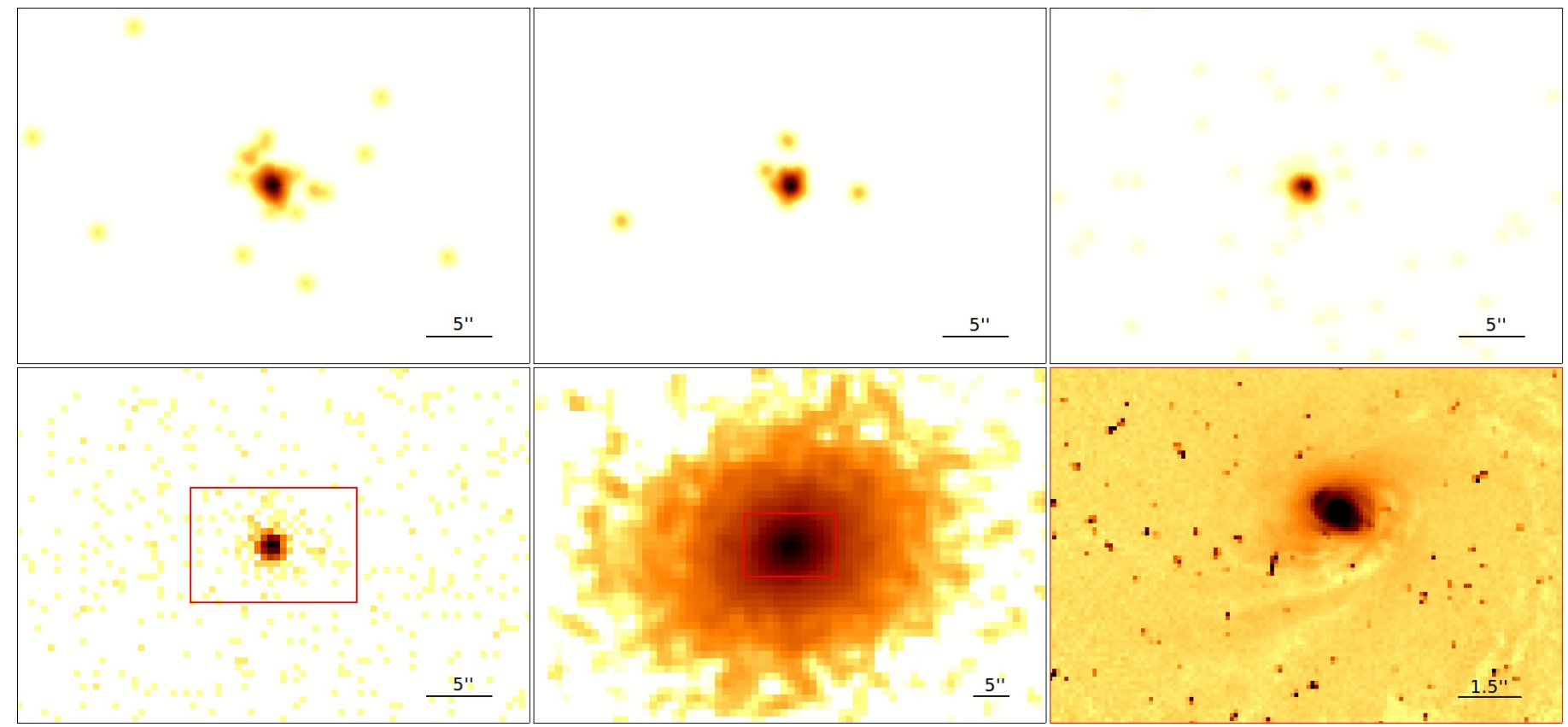

Fig. C.4. NGC 788. Top left: smoothed X-ray 0.6-0.9 keV energy band; top center: smoothed X-ray 1.6-2.0 keV energy band; top right: smoothed X-ray $4.5-8.0 \mathrm{keV}$ energy band; bottom left: X-ray $0.5-10.0 \mathrm{keV}$ energy band without smoothing; bottom center: 2MASS image in the $K_{\mathrm{s}}$ band; bottom right: Hubble image in the $F 606 \mathrm{~W}$ filter. 
L. Hernández-García et al.: X-ray variability of Seyfert 2s

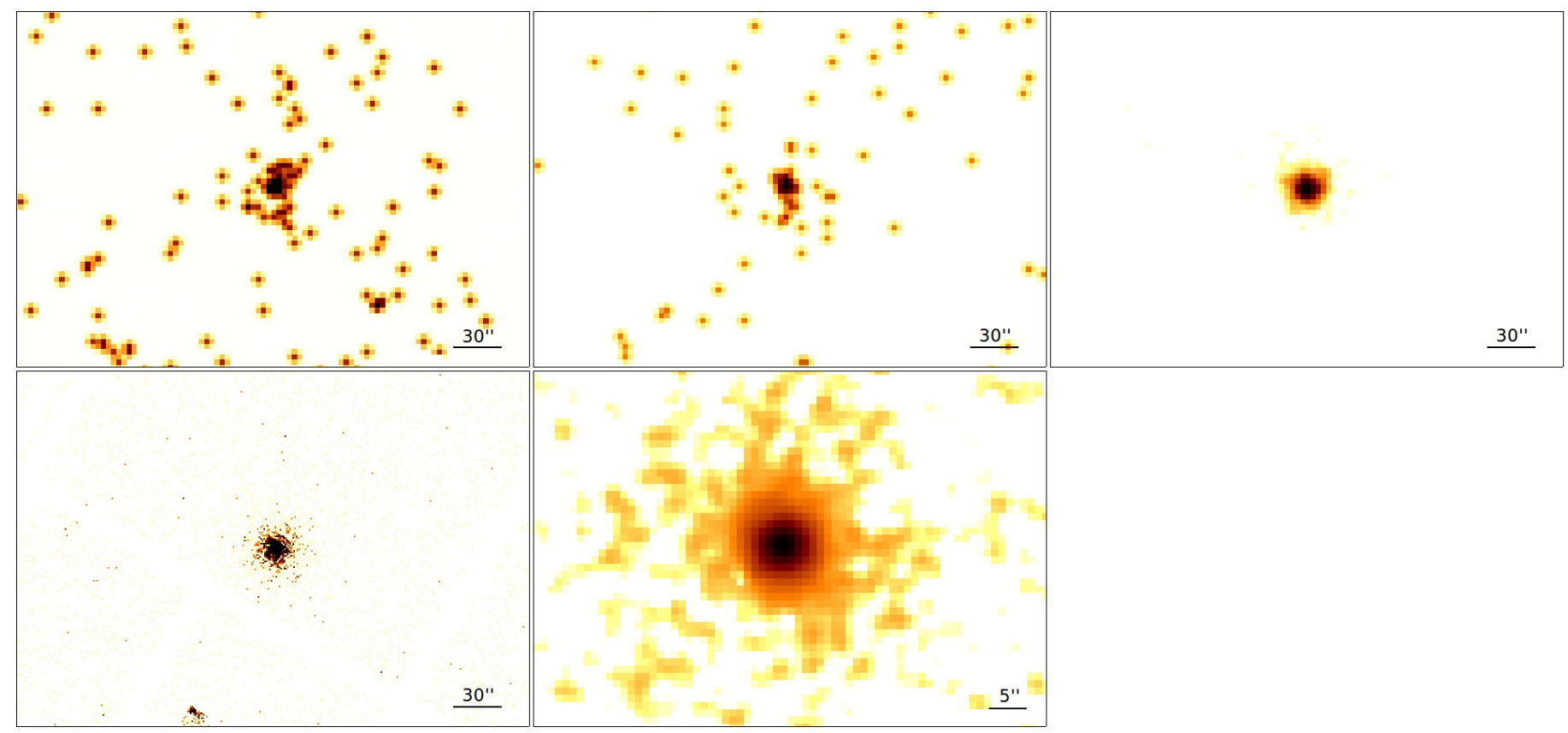

Fig. C.5. ESO 417-G06 (XMM-Newton data). Top left: smoothed X-ray 0.6-0.9 keV energy band; top center: smoothed X-ray 1.6-2.0 keV energy band; top right: smoothed X-ray 4.5-8.0 keV energy band; bottom left: X-ray $0.5-10.0 \mathrm{keV}$ energy band without smoothing; bottom center: 2MASS image in the $K_{\mathrm{s}}$ band.

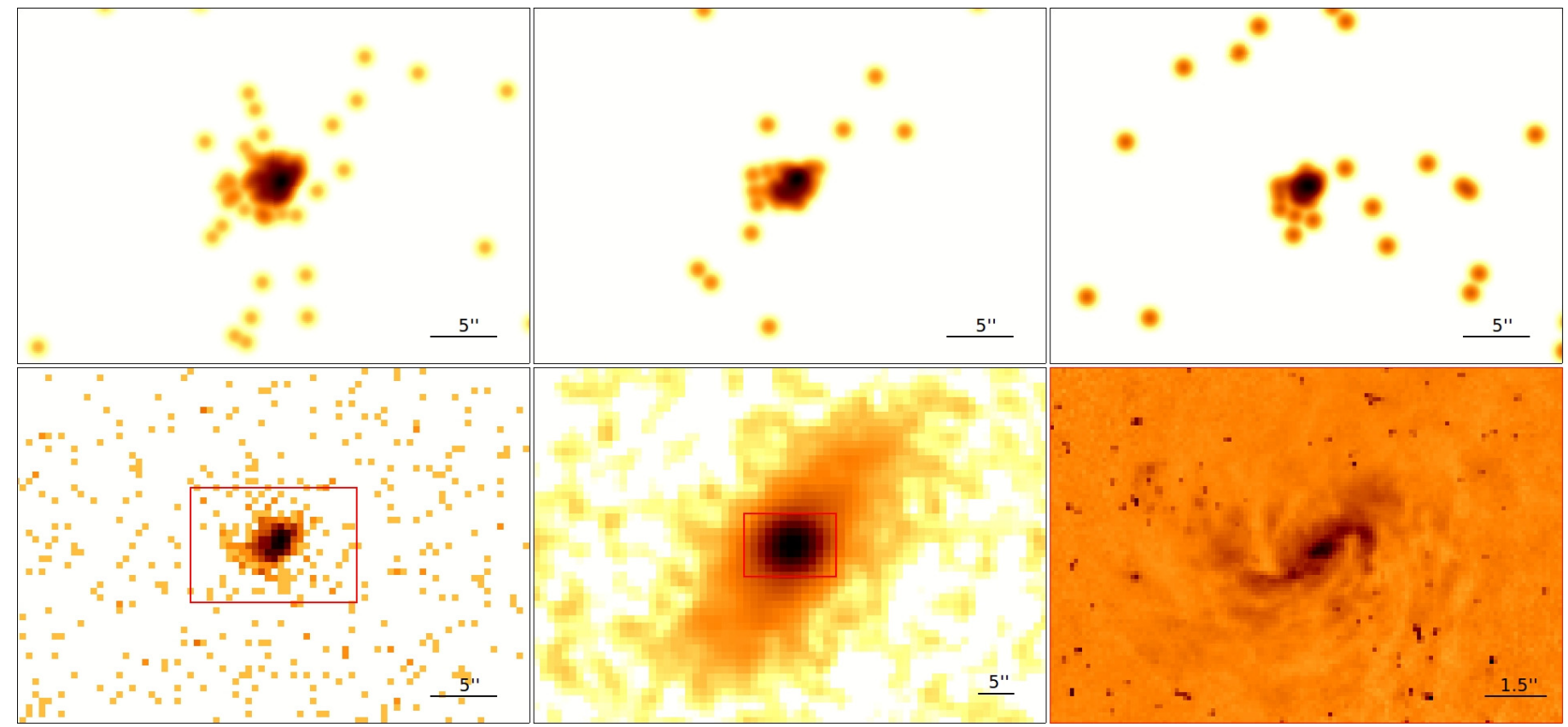

Fig. C.6. MARK 1066. Top left: smoothed X-ray 0.6-0.9 keV energy band; top center: smoothed X-ray 1.6-2.0 keV energy band; top right: smoothed X-ray 4.5-8.0 keV energy band; bottom left: X-ray 0.5-10.0 keV energy band without smoothing; bottom center: 2MASS image in the $K_{\mathrm{s}}$ band; bottom right: Hubble image in the $F 606 \mathrm{~W}$ filter. 
A\&A 579, A90 (2015)
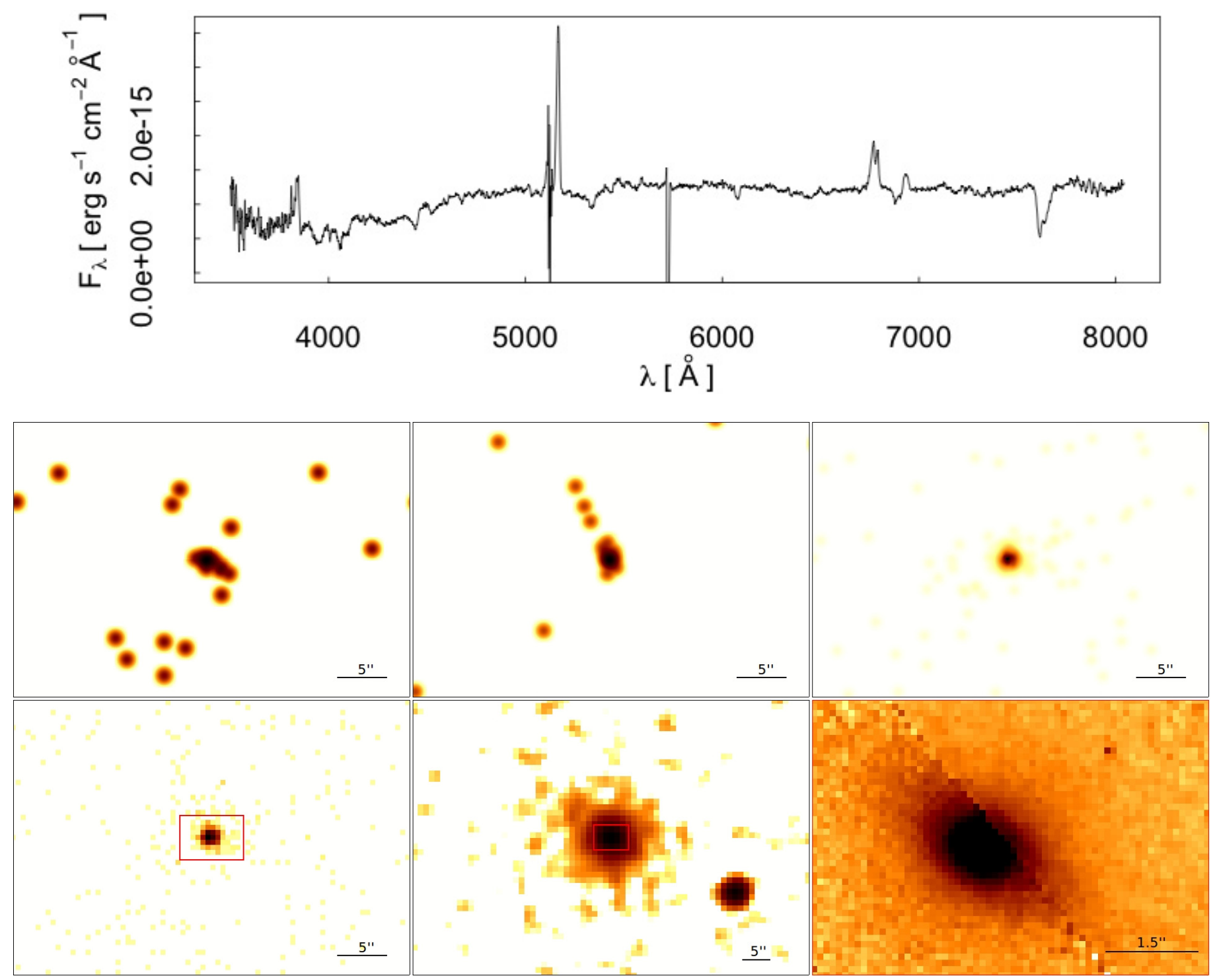

Fig. C.7. Up: optical spectrum (from NED); bottom: images of 3C 98.0. Top left: smoothed X-ray 0.6-0.9 keV energy band; top center: smoothed X-ray 1.6-2.0 keV energy band; top right: smoothed X-ray 4.5-8.0 keV energy band; bottom left: X-ray $0.5-10.0 \mathrm{keV}$ energy band without smoothing; bottom center: 2 MASS image in the $K_{\mathrm{s}}$ band; bottom right: Hubble image in the $F 606 \mathrm{~N}$ filter. 
L. Hernández-García et al.: X-ray variability of Seyfert $2 \mathrm{~s}$
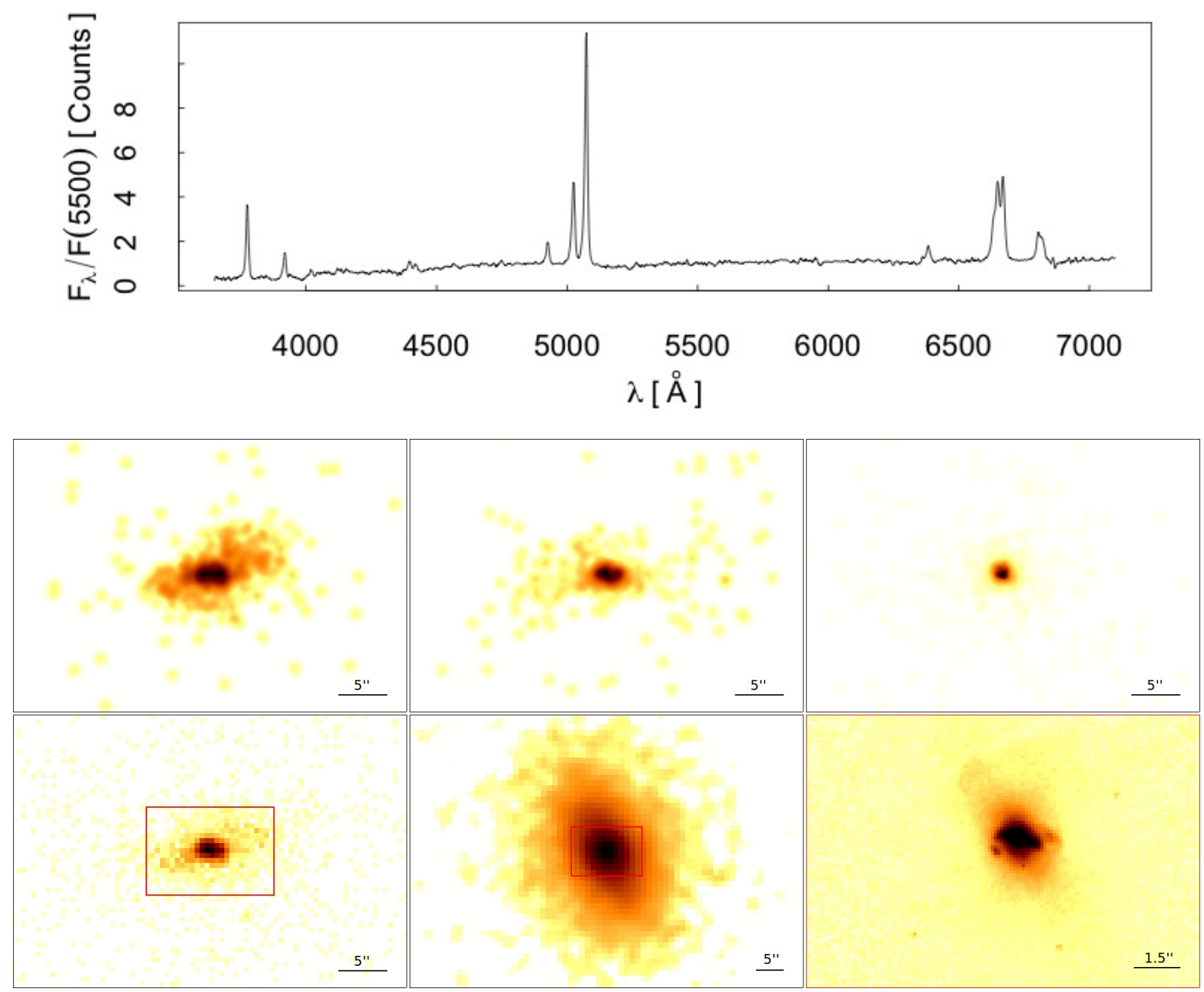

Fig. C.8. Up: optical spectrum (from NED); bottom: images of MARK 3. Top left: smoothed X-ray 0.6-0.9 keV energy band; top center: smoothed X-ray 1.6-2.0 keV energy band; top right: smoothed X-ray 4.5-8.0 keV energy band; bottom left: X-ray $0.5-10.0 \mathrm{keV}$ energy band without smoothing; bottom center: $2 \mathrm{MASS}$ image in the $K_{\mathrm{s}}$ band; bottom right: Hubble image in the $F 814 \mathrm{~W}$ filter. 
A\&A 579, A90 (2015)

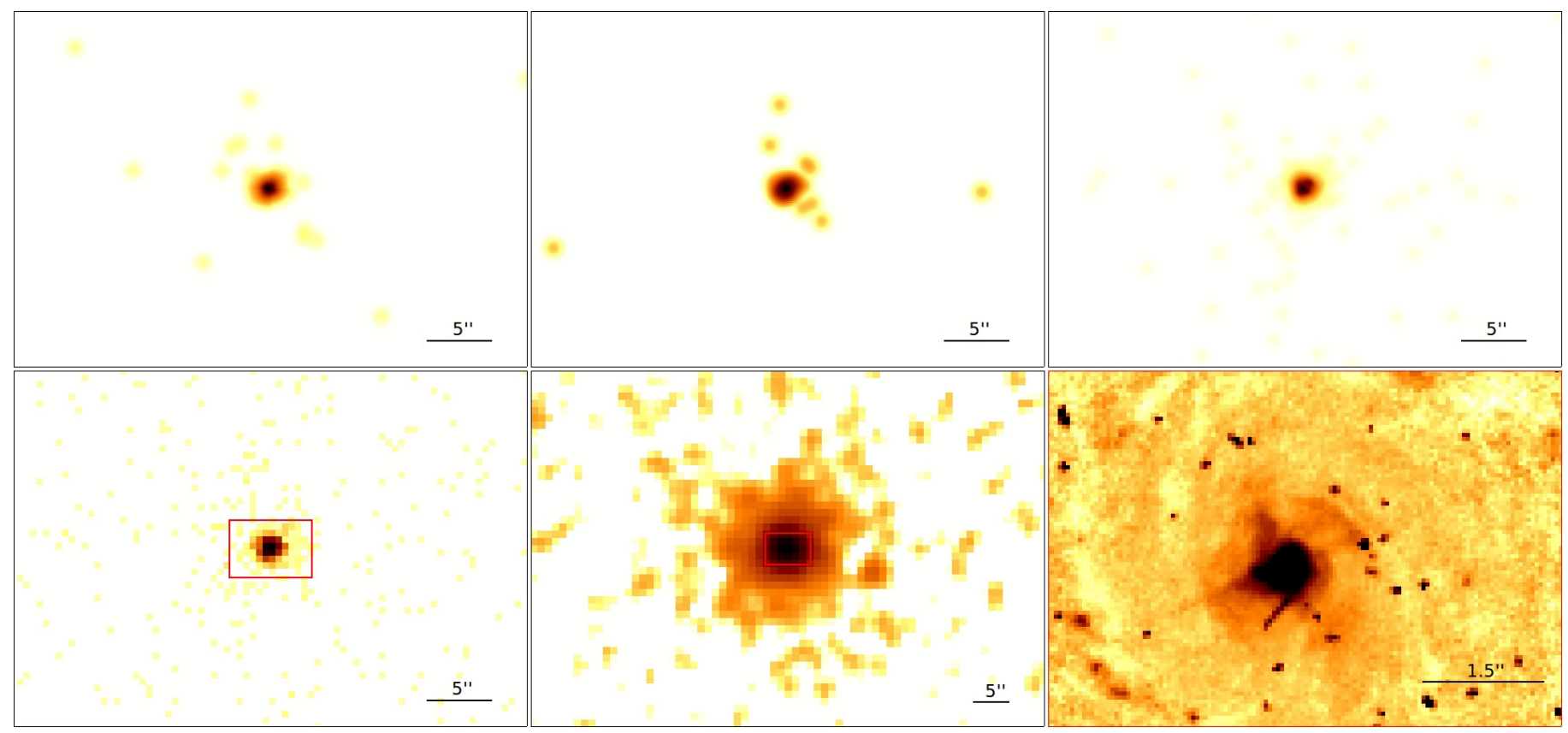

Fig. C.9. MARK 1210. Top left: smoothed X-ray 0.6-0.9 keV energy band; top center: smoothed X-ray 1.6-2.0 keV energy band; top right: smoothed X-ray $4.5-8.0 \mathrm{keV}$ energy band; bottom left: X-ray $0.5-10.0 \mathrm{keV}$ energy band without smoothing; bottom center: $2 \mathrm{MASS}$ image in the $K_{\mathrm{s}}$ band; bottom right: Hubble image in the $F 606 \mathrm{~W}$ filter. 
L. Hernández-García et al.: X-ray variability of Seyfert 2s
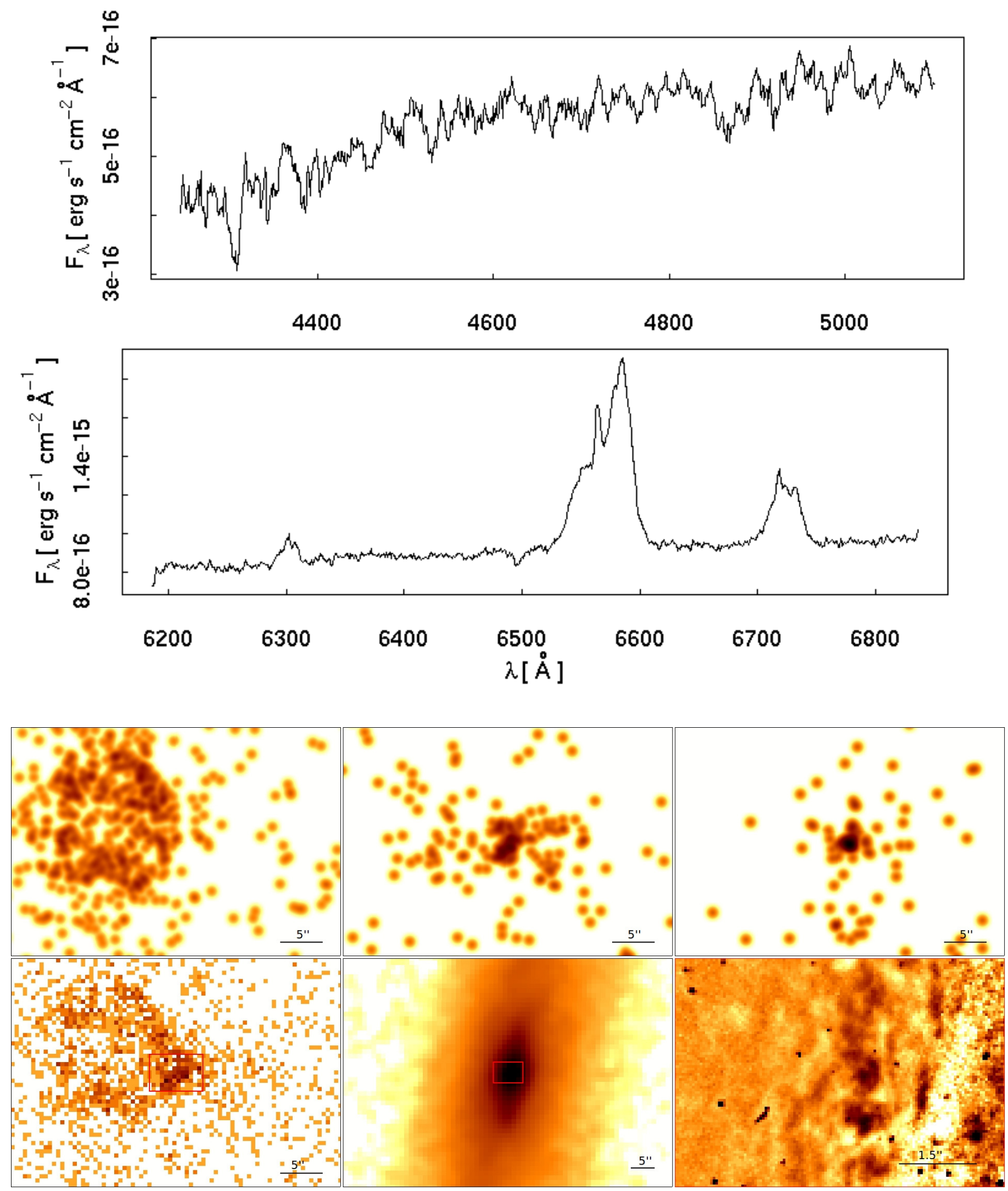

Fig. C.10. Up: optical spectra (from NED); bottom: images of NGC 3079. Top left: smoothed X-ray 0.6-0.9 keV energy band; top center: smoothed X-ray 1.6-2.0 keV energy band; top right: smoothed X-ray 4.5-8.0 keV energy band; bottom left: X-ray $0.5-10.0 \mathrm{keV}$ energy band without smoothing; bottom center: $2 \mathrm{MASS}$ image in the $K_{\mathrm{s}}$ band; bottom right: Hubble image in the $F 814 \mathrm{~W}$ filter. 

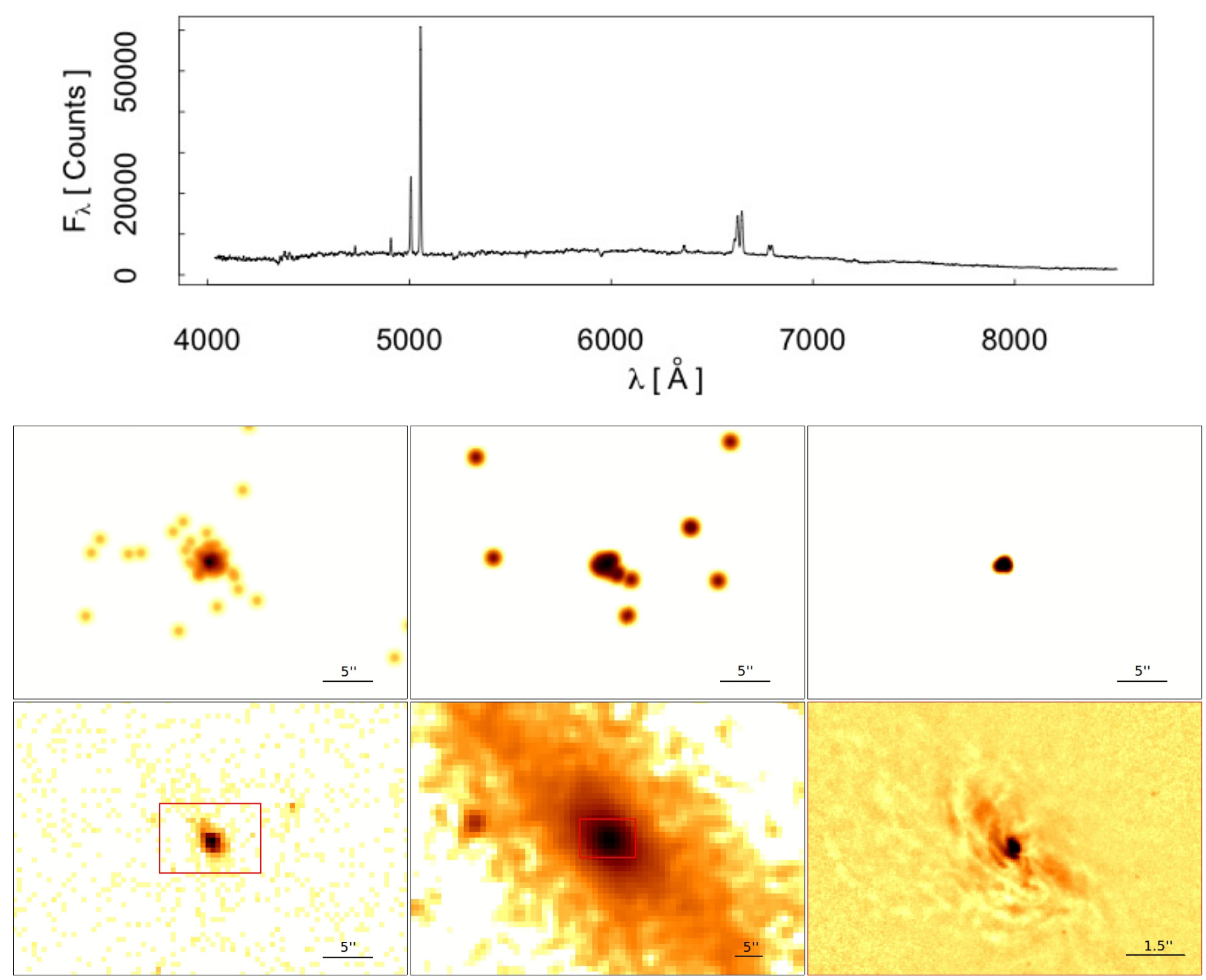

Fig. C.11. Up: optical spectrum (from NED); bottom: images of IC 2560. Top left: smoothed X-ray 0.6-0.9 keV energy band; top center: smoothed X-ray 1.6-2.0 keV energy band; top right: smoothed X-ray 4.5-8.0 keV energy band; bottom left: X-ray $0.5-10.0 \mathrm{keV}$ energy band without smoothing; bottom center: 2MASS image in the $K_{\mathrm{s}}$ band; bottom right: Hubble image in the $F 814 \mathrm{~W}$ filter. 
L. Hernández-García et al.: X-ray variability of Seyfert 2s
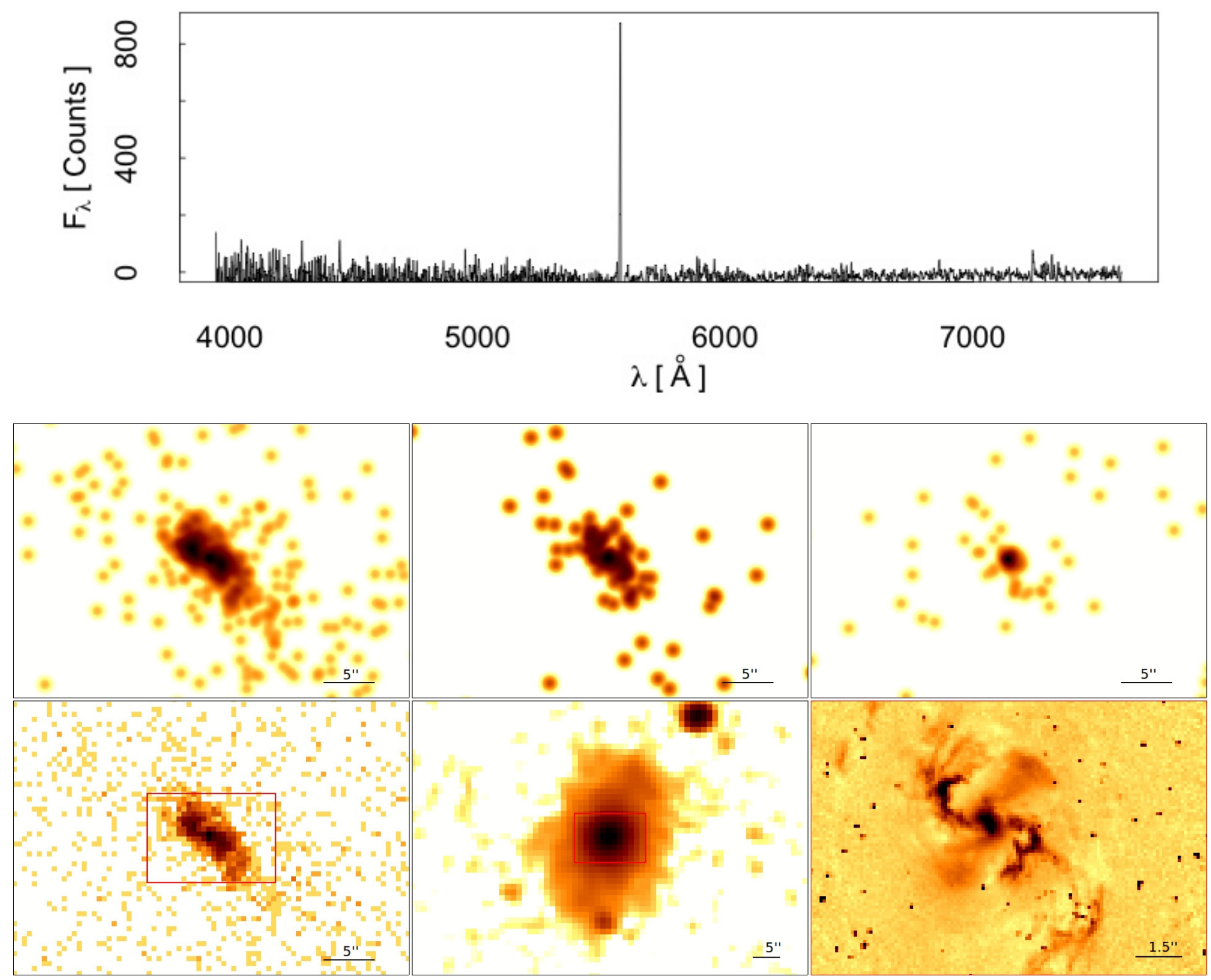

Fig. C.12. Up: optical spectrum (from NED); bottom: images of NGC 3393. Top left: smoothed X-ray 0.6-0.9 keV energy band; top center: smoothed X-ray 1.6-2.0 keV energy band; top right: smoothed X-ray 4.5-8.0 keV energy band; bottom left: X-ray 0.5-10.0 keV energy band without smoothing; bottom center: $2 \mathrm{MASS}$ image in the $K_{\mathrm{s}}$ band; bottom right: Hubble image in the $F 606 \mathrm{~W}$ filter. 
A\&A 579, A90 (2015)
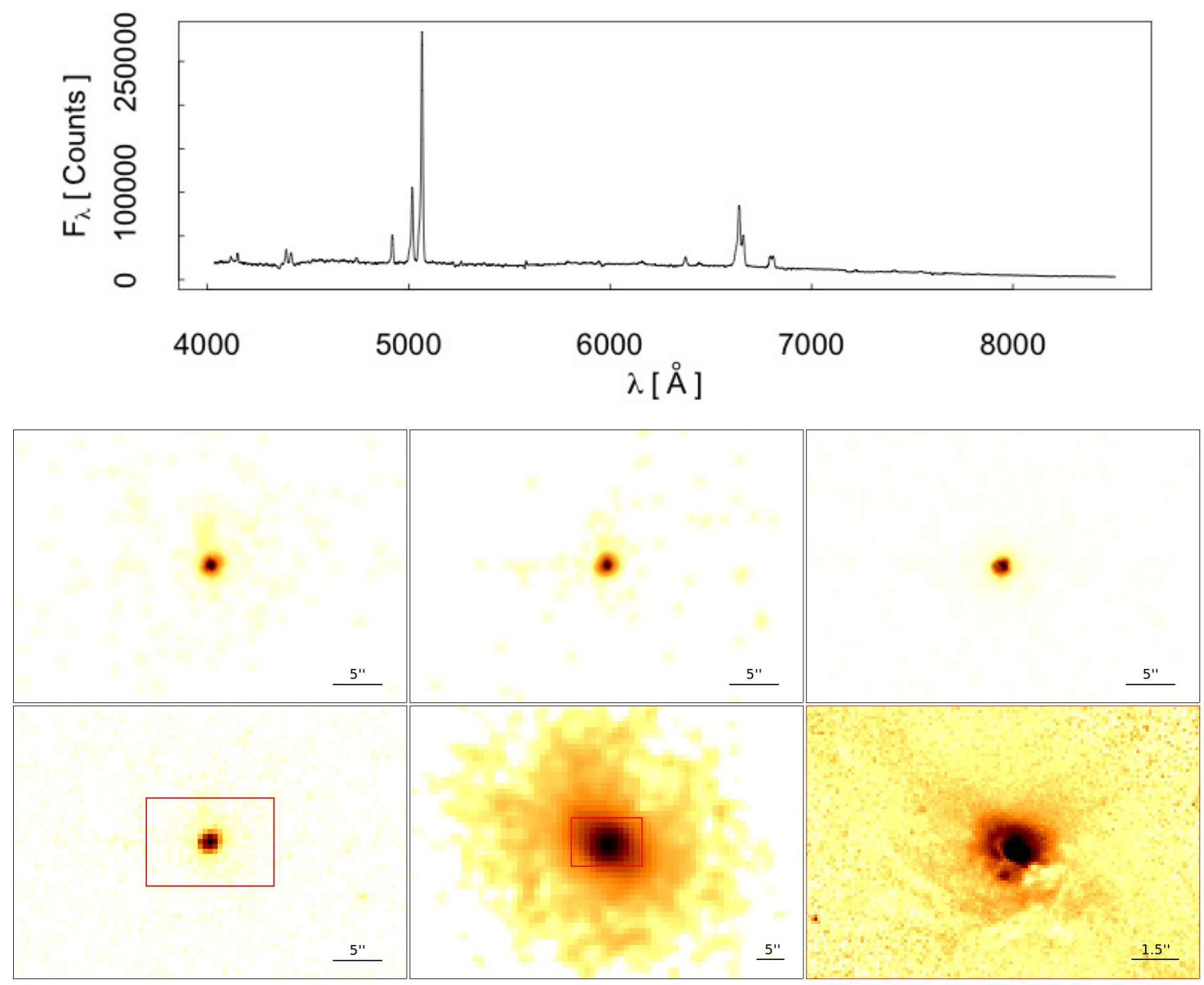

Fig. C.13. Up: optical spectrum (from NED); bottom: images of NGC 4507. Top left: smoothed X-ray 0.6-0.9 keV energy band; top center: smoothed X-ray 1.6-2.0 keV energy band; top right: smoothed X-ray 4.5-8.0 keV energy band; bottom left: X-ray $0.5-10.0 \mathrm{keV}$ energy band without smoothing; bottom center: $2 \mathrm{MASS}$ image in the $K_{\mathrm{s}}$ band; bottom right: Hubble image in the $F 814 W$ filter. 
L. Hernández-García et al.: X-ray variability of Seyfert 2s
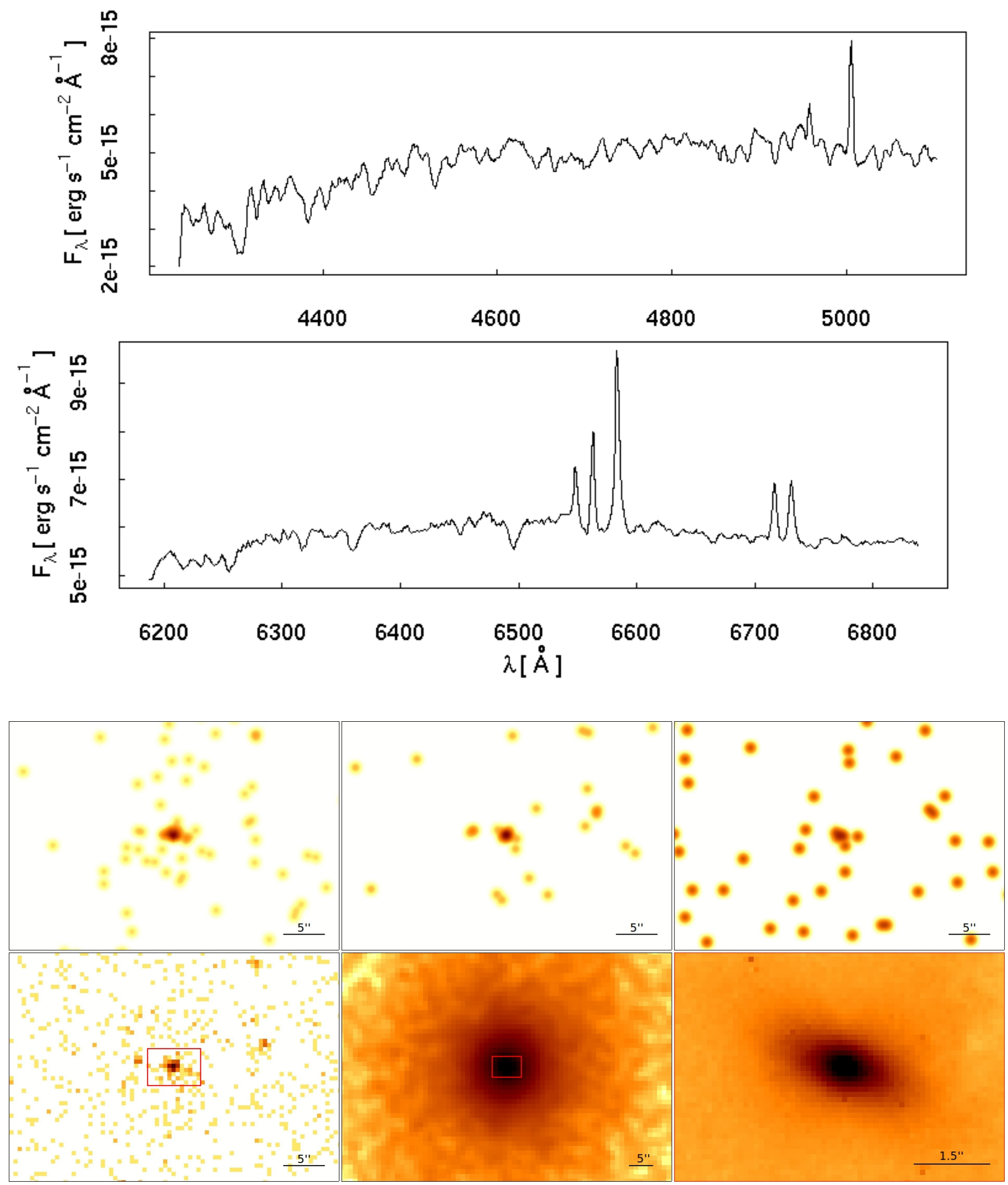

Fig. C.14. Up: optical spectra (from NED); bottom: images of NGC 4698. Top left: smoothed X-ray 0.6-0.9 keV energy band; top center: smoothed X-ray 1.6-2.0 keV energy band; top right: smoothed X-ray 4.5-8.0 keV energy band; bottom left: X-ray $0.5-10.0 \mathrm{keV}$ energy band without smoothing; bottom center: $2 \mathrm{MASS}$ image in the $K_{\mathrm{s}}$ band; bottom right: Hubble image in the $F 814 \mathrm{~W}$ filter. 

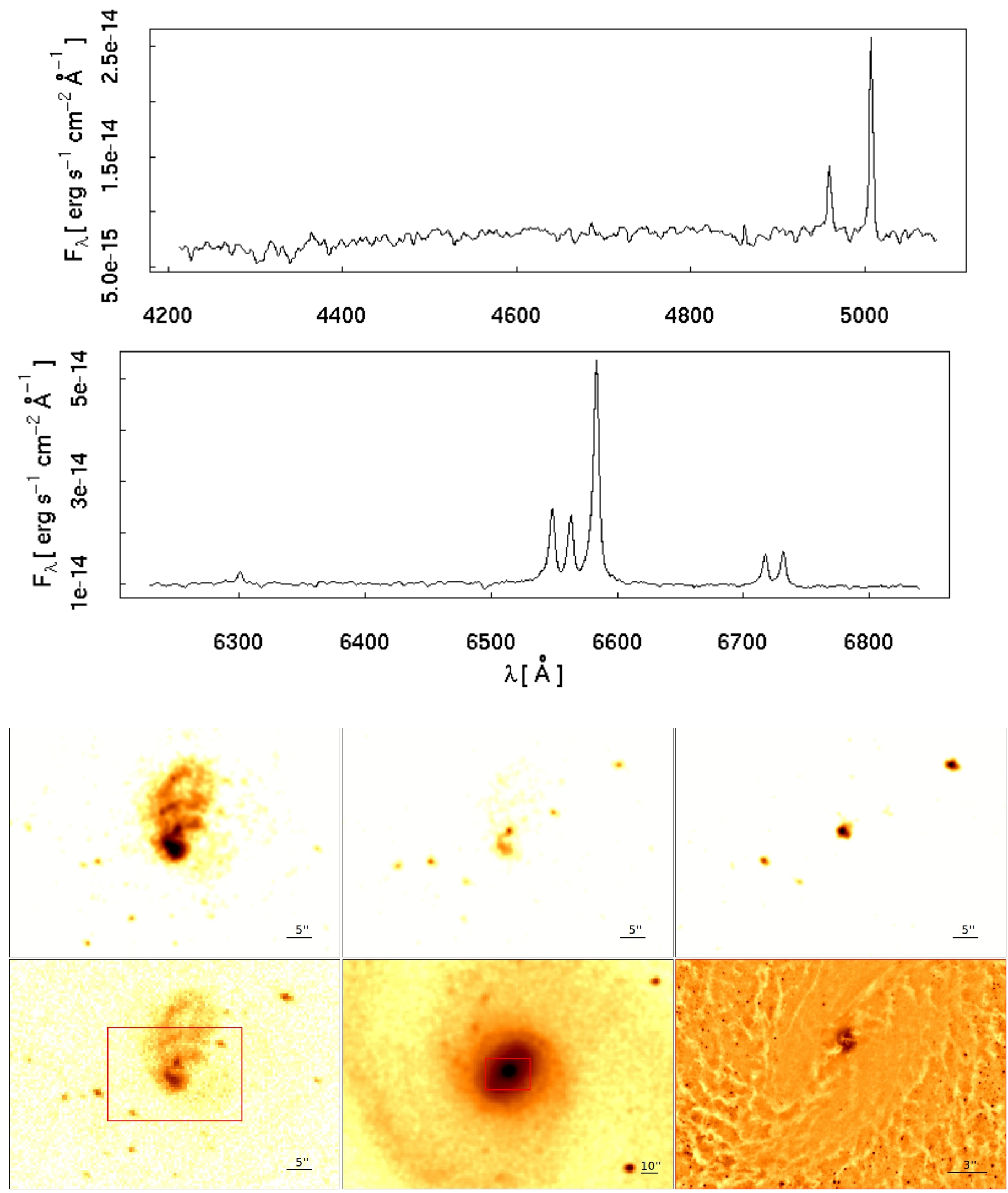

Fig. C.15. Up: optical spectra (from NED); bottom: images of NGC 5194. Top left: smoothed X-ray 0.6-0.9 keV energy band; top center: smoothed X-ray 1.6-2.0 keV energy band; top right: smoothed X-ray 4.5-8.0 keV energy band; bottom left: X-ray $0.5-10.0 \mathrm{keV}$ energy band without smoothing; bottom center: 2 MASS image in the $K_{\mathrm{s}}$ band; bottom right: Hubble image in the $F 814 \mathrm{~W}$ filter. 
L. Hernández-García et al.: X-ray variability of Seyfert 2s

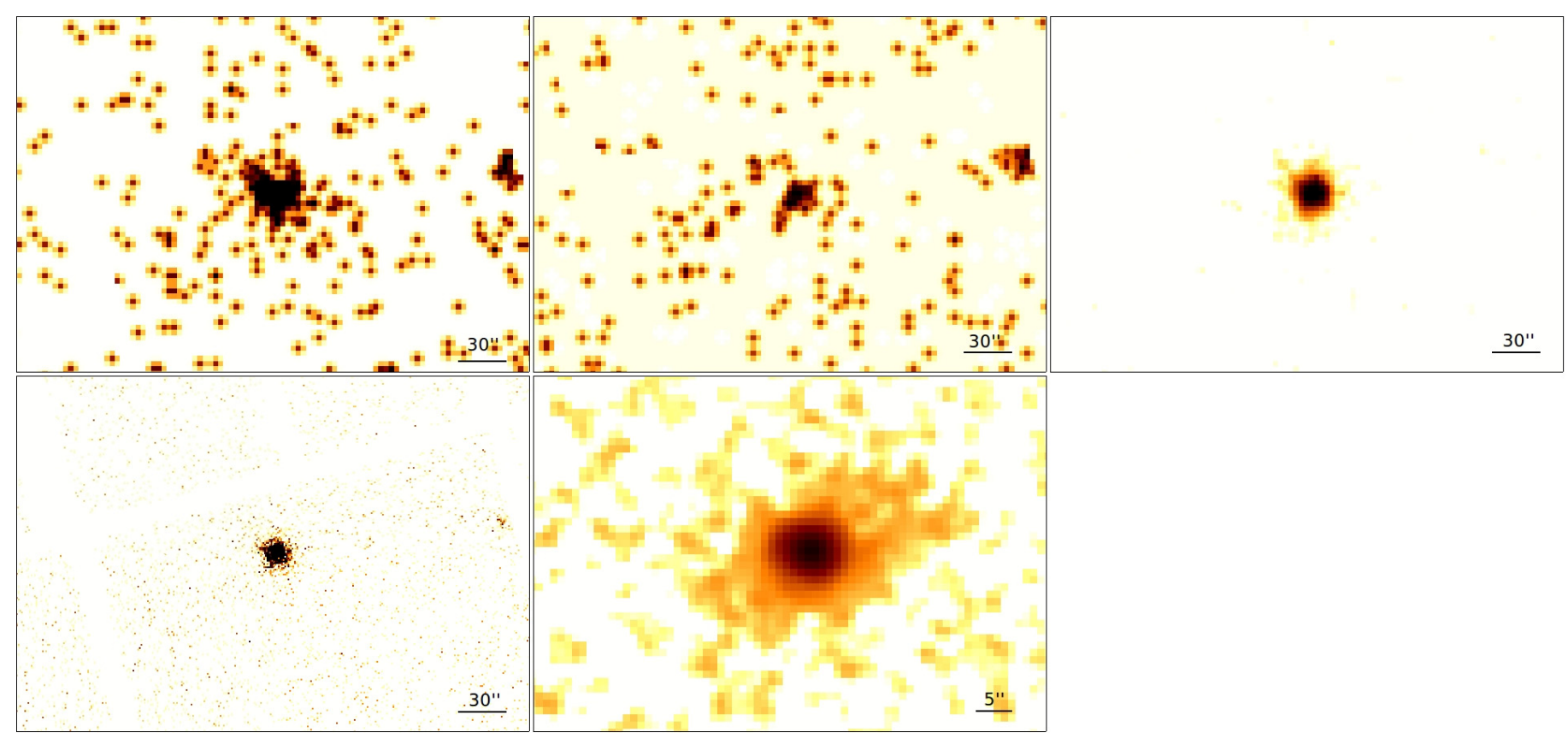

Fig. C.16. MARK 268 (XMM-Newton data). Top left: smoothed X-ray 0.6-0.9 keV energy band; top center: smoothed X-ray 1.6-2.0 keV energy band; top right: smoothed X-ray 4.5-8.0 keV energy band; bottom left: X-ray $0.5-10.0 \mathrm{keV}$ energy band without smoothing; bottom center: 2MASS image in the $K_{\mathrm{s}}$ band. 

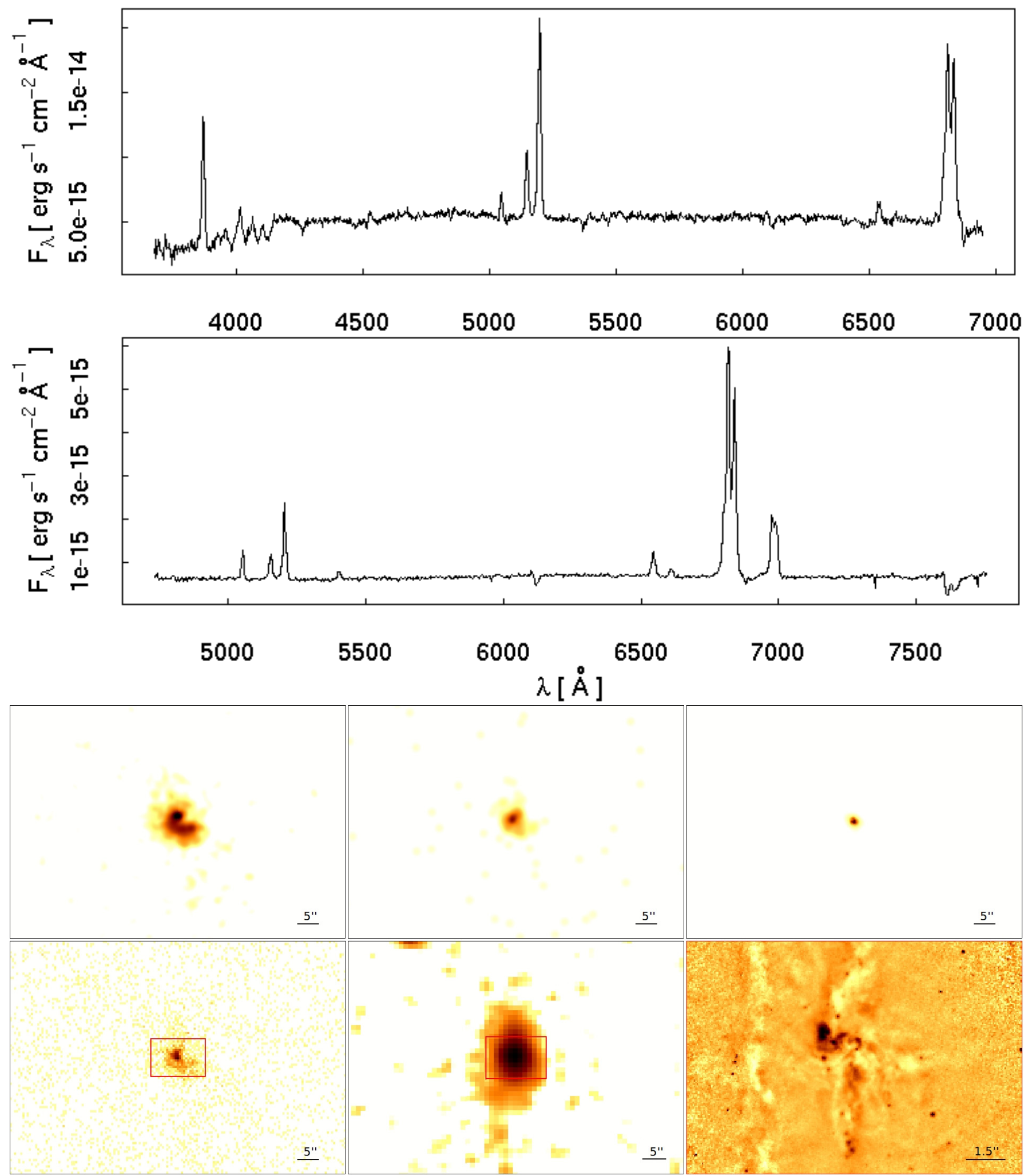

Fig. C.17. Up: optical spectra (from NED); bottom: images of MARK 273. Top left: smoothed X-ray 0.6-0.9 keV energy band; top center: smoothed X-ray 1.6-2.0 keV energy band; top right: smoothed X-ray 4.5-8.0 keV energy band; bottom left: X-ray $0.5-10.0 \mathrm{keV}$ energy band without smoothing; bottom center: $2 \mathrm{MASS}$ image in the $K_{\mathrm{s}}$ band; bottom right: Hubble image in the $F 814 \mathrm{~W}$ filter. 
L. Hernández-García et al.: X-ray variability of Seyfert 2s

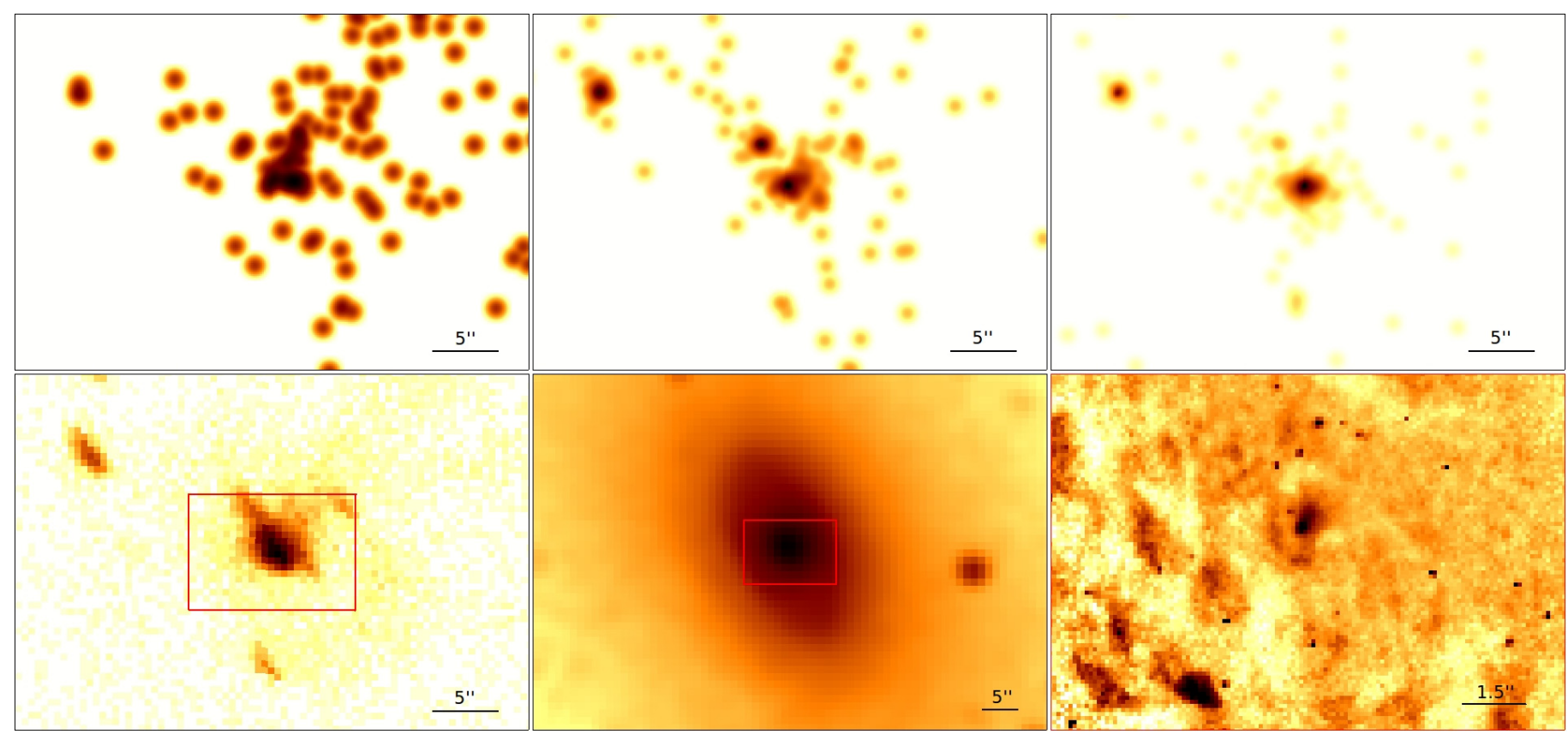

Fig. C.18. Circinus. Top left: smoothed X-ray 0.6-0.9 keV energy band; top center: smoothed X-ray $1.6-2.0 \mathrm{keV}$ energy band; top right: smoothed X-ray $4.5-8.0 \mathrm{keV}$ energy band; bottom left: X-ray $0.5-10.0 \mathrm{keV}$ energy band without smoothing; bottom center: $2 \mathrm{MASS}$ image in the $K_{\mathrm{s}}$ band; bottom right: Hubble image in the $F 814 W$ filter. 

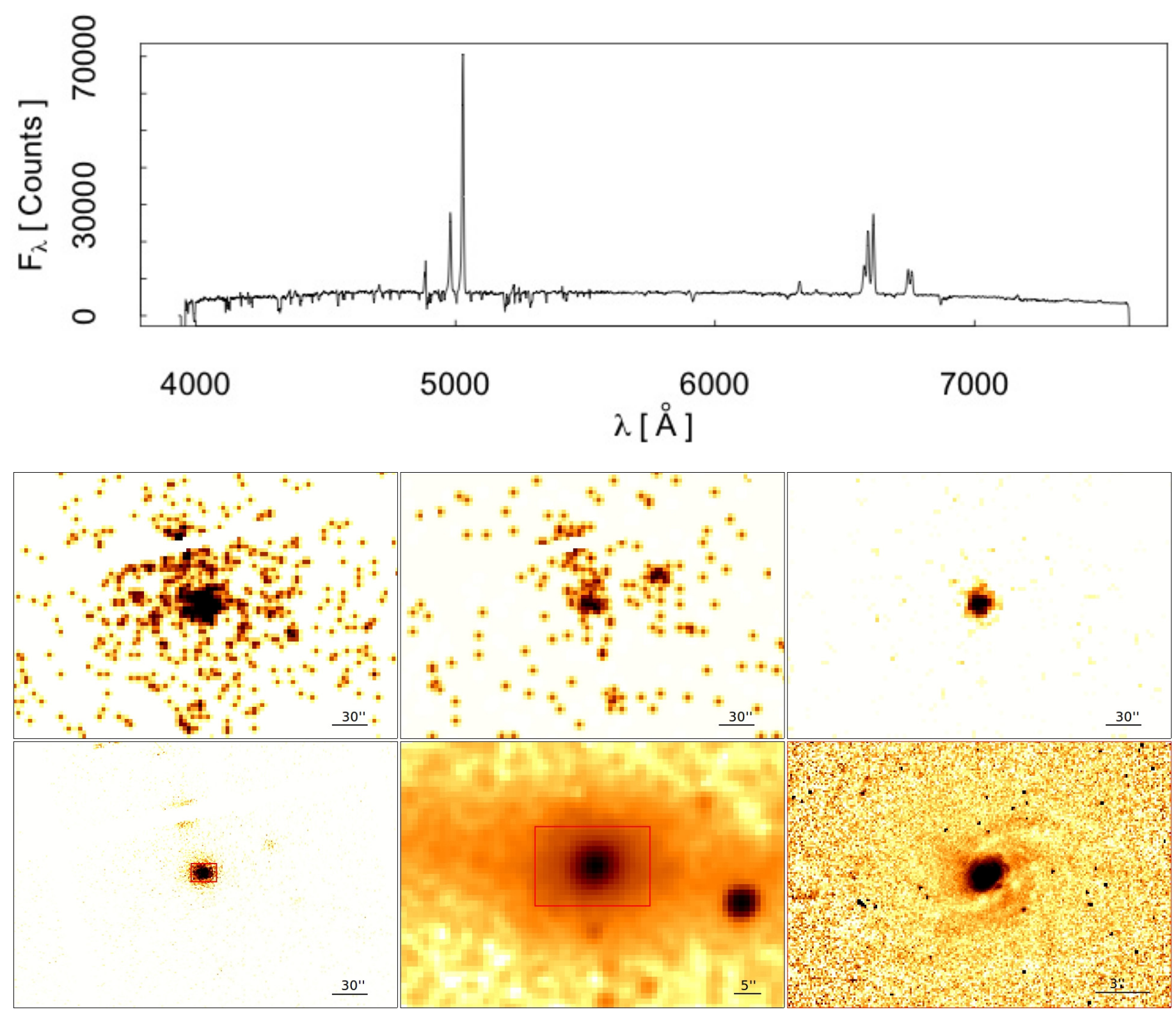

Fig. C.19. Up: optical spectrum (from NED), and bottom: images of NGC 5643 (XMM-Newton data). Top left: smoothed X-ray 0.6-0.9 keV energy band; top center: smoothed X-ray 1.6-2.0 keV energy band; top right: smoothed X-ray 4.5-8.0 keV energy band; bottom left: X-ray $0.5-10.0 \mathrm{keV}$ energy band without smoothing; bottom center: 2MASS image in the $K_{\mathrm{s}}$ band; bottom right: Hubble image in the $F 814 \mathrm{~W}$ filter. 
L. Hernández-García et al.: X-ray variability of Seyfert $2 \mathrm{~s}$

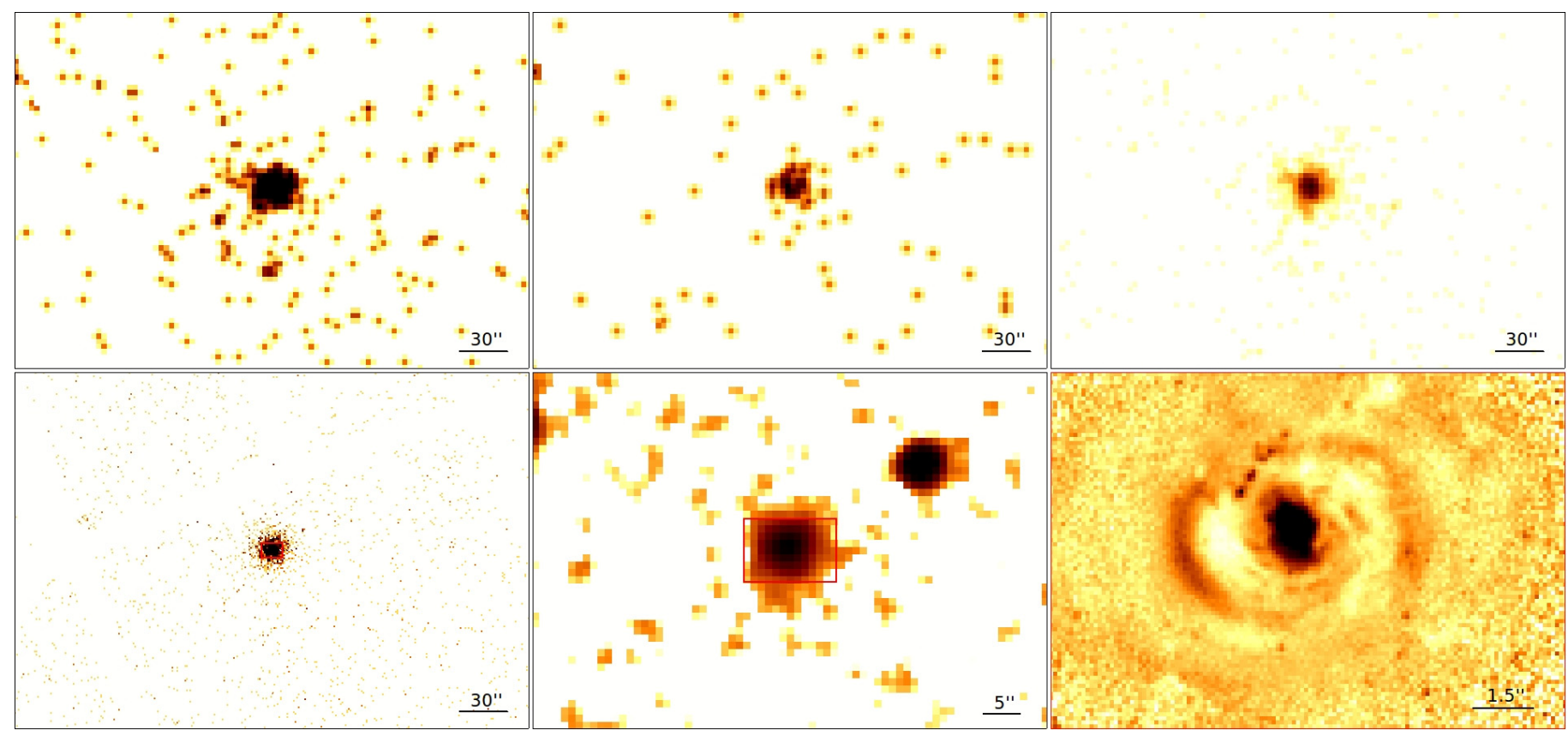

Fig. C.20. MARK 477 (XMM-Newton data). Top left: smoothed X-ray 0.6-0.9 keV energy band; top center: smoothed X-ray 1.6-2.0 keV energy band; top right: smoothed X-ray $4.5-8.0 \mathrm{keV}$ energy band; bottom left: X-ray $0.5-10.0 \mathrm{keV}$ energy band without smoothing; bottom center: 2MASS image in the $K_{\mathrm{s}}$ band; bottom right: Hubble image in the $F 606 \mathrm{~W}$ filter.

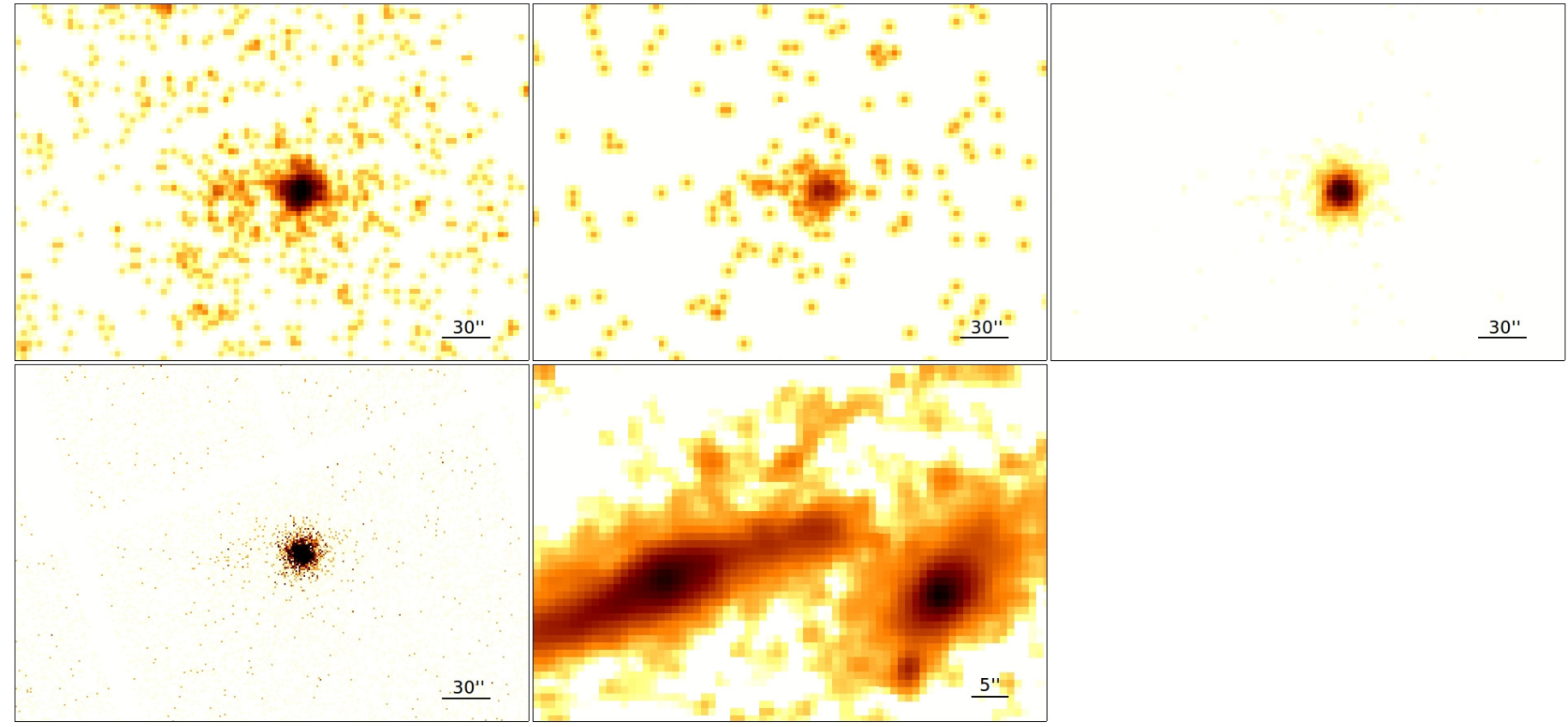

Fig. C.21. IC 4518A (XMM-Newton data).Top left: smoothed X-ray 0.6-0.9 keV energy band; top center: smoothed X-ray 1.6-2.0 keV energy band; top right: smoothed X-ray 4.5-8.0 keV energy band; bottom left: X-ray 0.5-10.0 keV energy band without smoothing; bottom center: 2MASS image in the $K_{\mathrm{s}}$ band. 


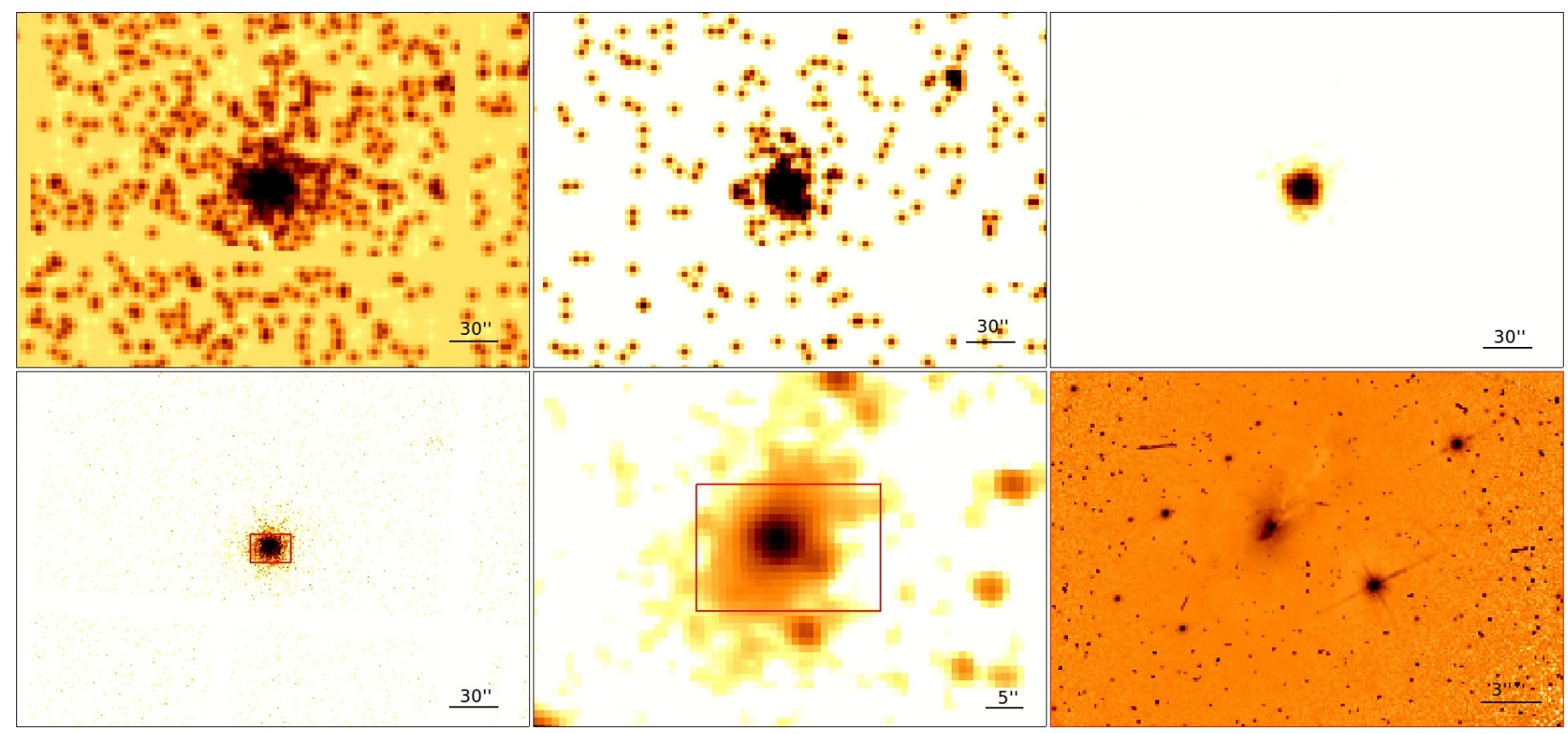

Fig. C.22. ESO 138-G01 (XMM-Newton data). Top left: smoothed X-ray 0.6-0.9 keV energy band; top center: smoothed X-ray 1.6-2.0 keV energy band; top right: smoothed X-ray $4.5-8.0 \mathrm{keV}$ energy band; bottom left: X-ray $0.5-10.0 \mathrm{keV}$ energy band without smoothing; bottom center: 2MASS image in the $K_{\mathrm{s}}$ band; bottom right: Hubble image in the $F 606 \mathrm{~W}$ filter.

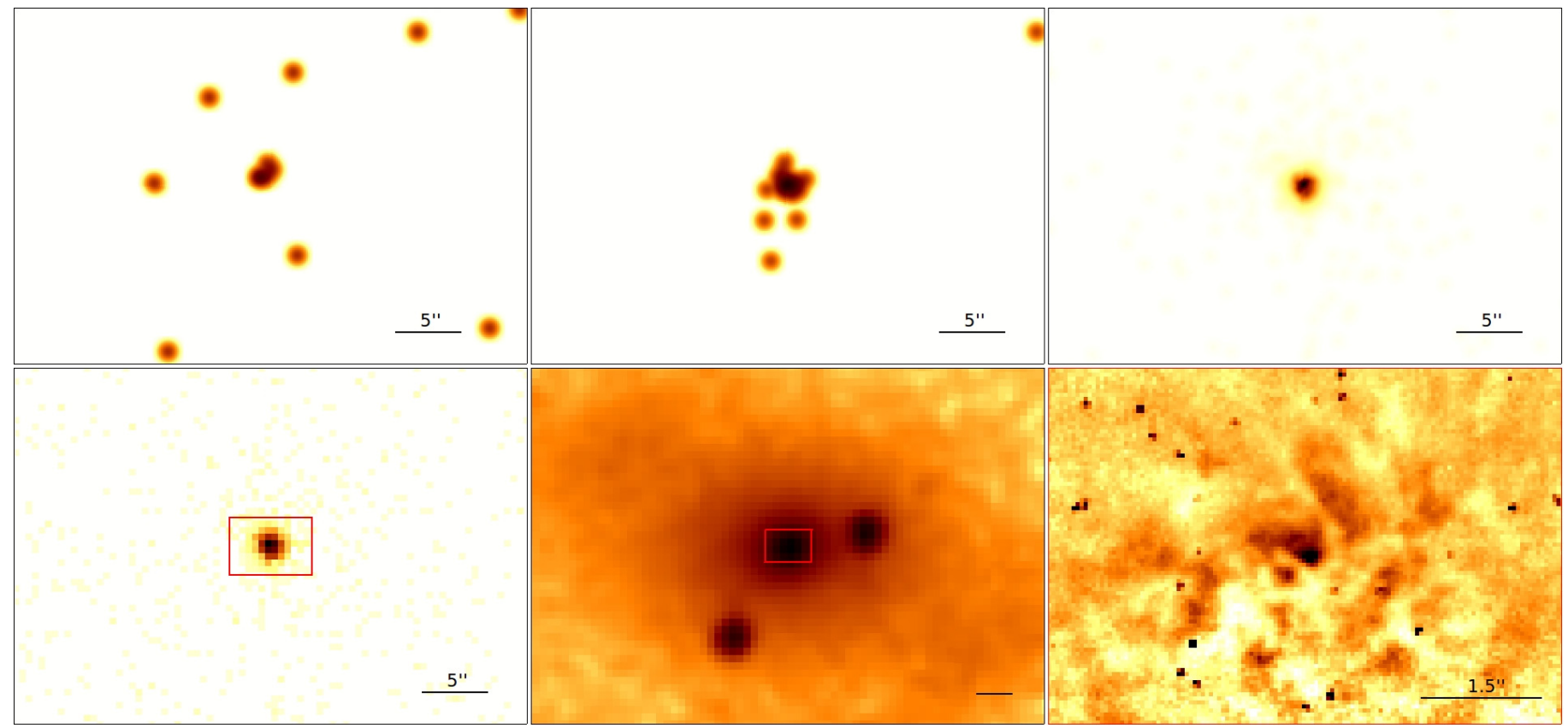

Fig. C.23. NGC 6300. Top left: smoothed X-ray $0.6-0.9 \mathrm{keV}$ energy band; top center: smoothed X-ray $1.6-2.0 \mathrm{keV}$ energy band; top right: smoothed X-ray 4.5-8.0 keV energy band; bottom left: X-ray 0.5-10.0 keV energy band without smoothing; bottom center: 2MASS image in the $K_{\mathrm{s}}$ band; bottom right: Hubble image in the $F 606 \mathrm{~W}$ filter. 
L. Hernández-García et al.: X-ray variability of Seyfert 2s
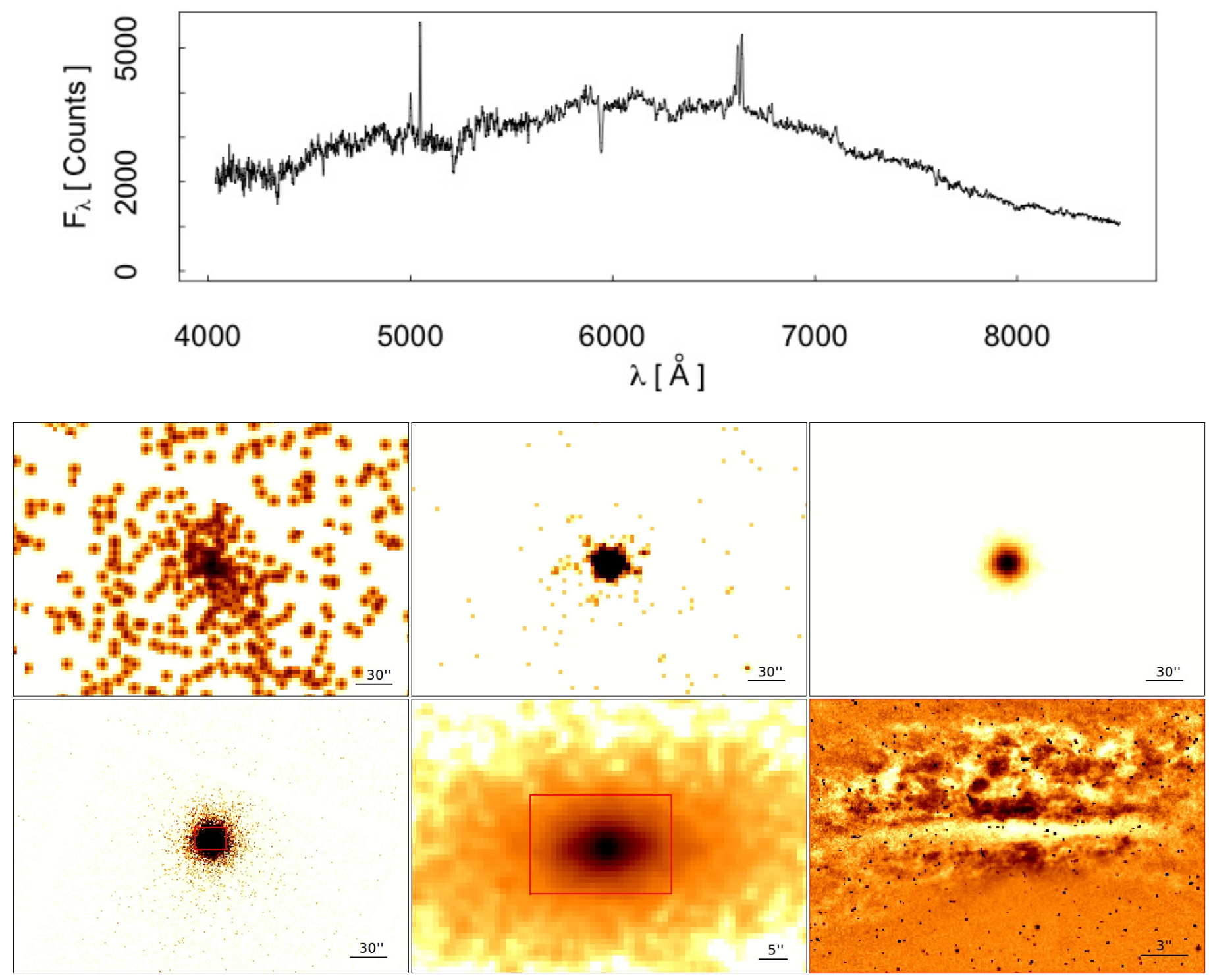

Fig. C.24. Up: optical spectrum (from NED), and bottom: images of NGC 7172 (XMM-Newton data). Top left: smoothed X-ray 0.6-0.9 keV energy band; top center: smoothed X-ray 1.6-2.0 keV energy band; top right: smoothed X-ray $4.5-8.0 \mathrm{keV}$ energy band; bottom left: X-ray $0.5-10.0 \mathrm{keV}$ energy band without smoothing; bottom center: 2 MASS image in the $K_{\mathrm{s}}$ band; bottom right: Hubble image in the $F 606 \mathrm{~W}$ filter. 

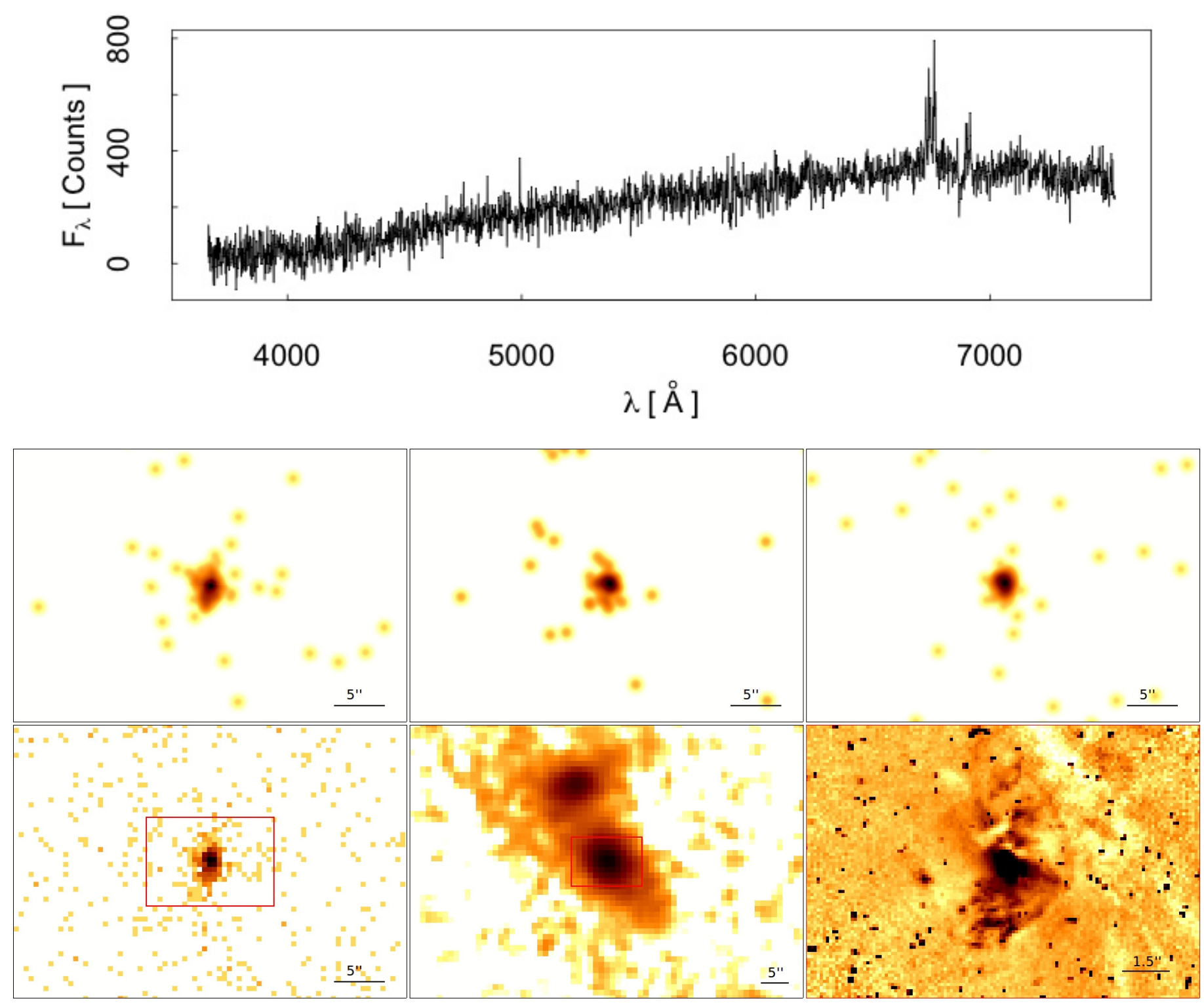

Fig. C.25. Up: optical spectrum (from NED), and bottom: images of NGC 7212. Top left: smoothed X-ray 0.6-0.9 keV energy band; top center: smoothed X-ray 1.6-2.0 keV energy band; top right: smoothed X-ray 4.5-8.0 keV energy band; bottom left: X-ray $0.5-10.0 \mathrm{keV}$ energy band without smoothing; bottom center: 2 MASS image in the $K_{\mathrm{s}}$ band; bottom right: Hubble image in the $F 606 W$ filter. 

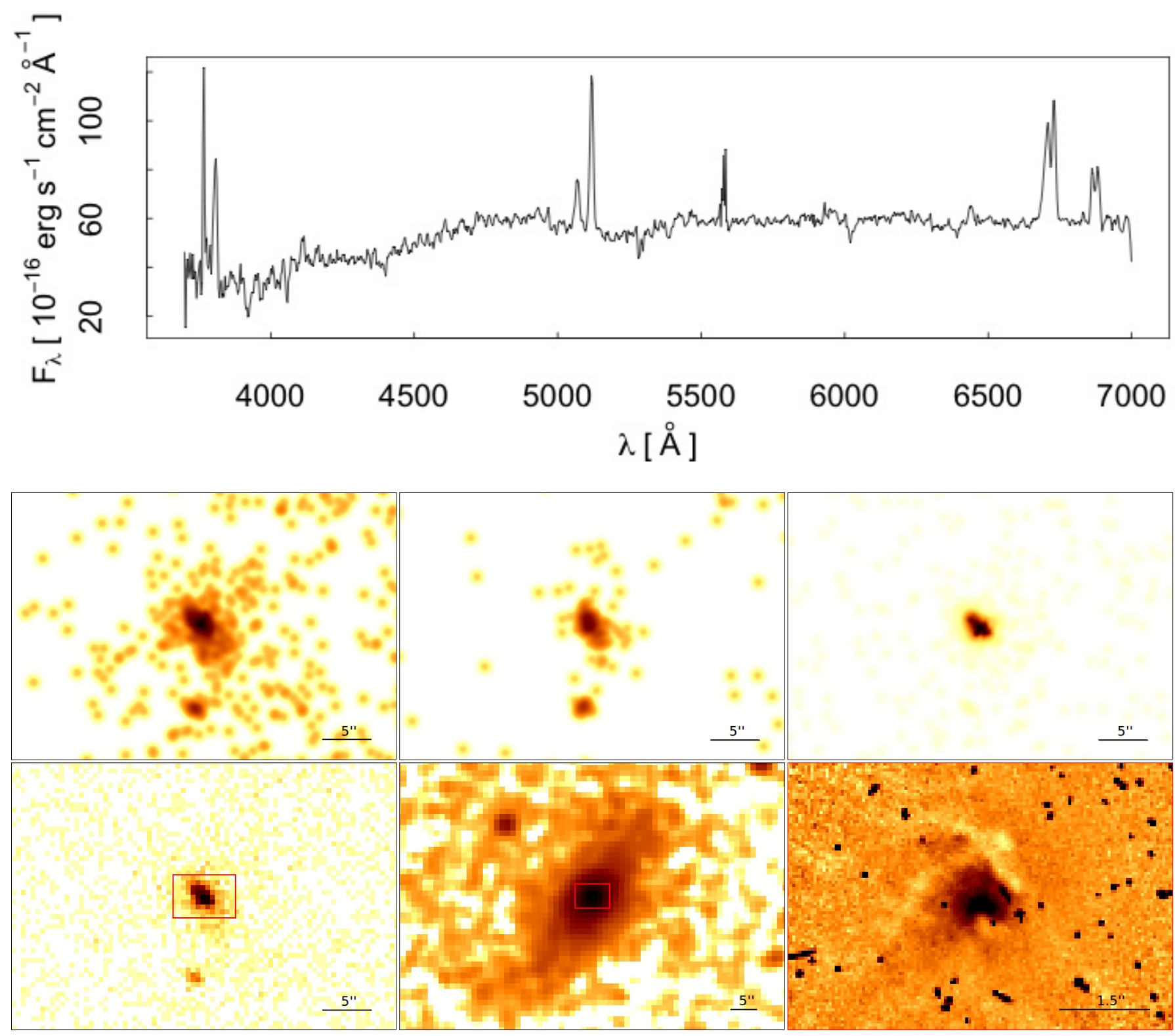

Fig. C.26. Up: optical spectrum (from NED), and bottom: images of NGC 7319. Top left: smoothed X-ray 0.6-0.9 keV energy band; top center: smoothed X-ray 1.6-2.0 keV energy band; top right: smoothed X-ray 4.5-8.0 keV energy band; bottom left: X-ray $0.5-10.0 \mathrm{keV}$ energy band without smoothing; bottom center: $2 \mathrm{MASS}$ image in the $K_{\mathrm{s}}$ band; bottom right: Hubble image in the $F 814 \mathrm{~W}$ filter.

\section{C.2. Chandra and XMM-Newton images}

In this appendix we present the images from Chandra (left) and XMM-Newton (right) that were used to compare the spectra from these two instruments in the $0.5-10 \mathrm{keV}$ band. In all cases, the gray scales extend from twice the value of the background dispersion to the maximum value at the center of each galaxy. 


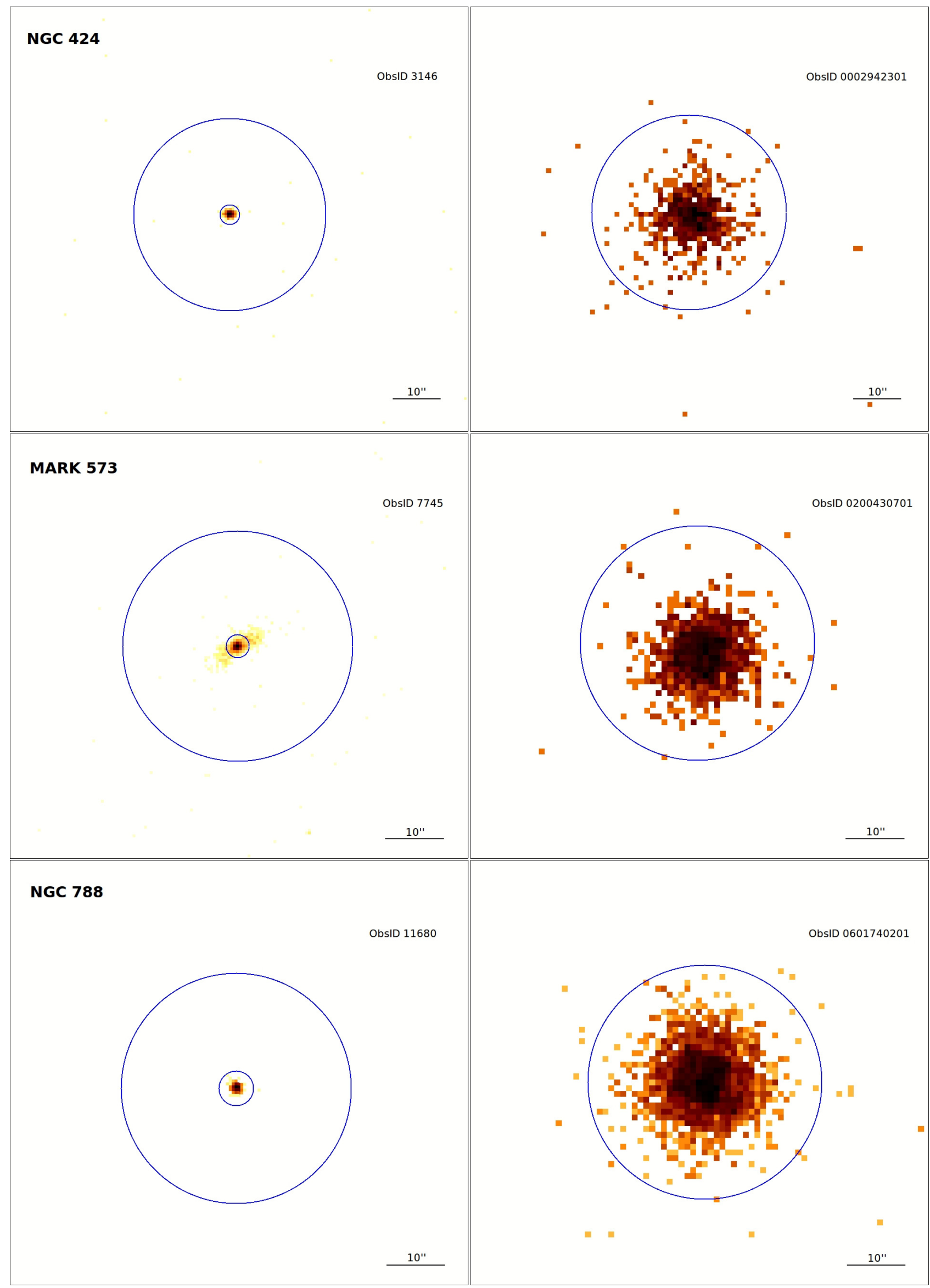

Fig. C.27. Chandra data left and XMM-Newton data right for the sources in the $0.5-10 \mathrm{keV}$ band. Big circles represent XMM-Newton data apertures. Small circles in the figures to the left represent the nuclear extraction aperture used with Chandra observations (see Table A.1). 
L. Hernández-García et al.: X-ray variability of Seyfert 2s

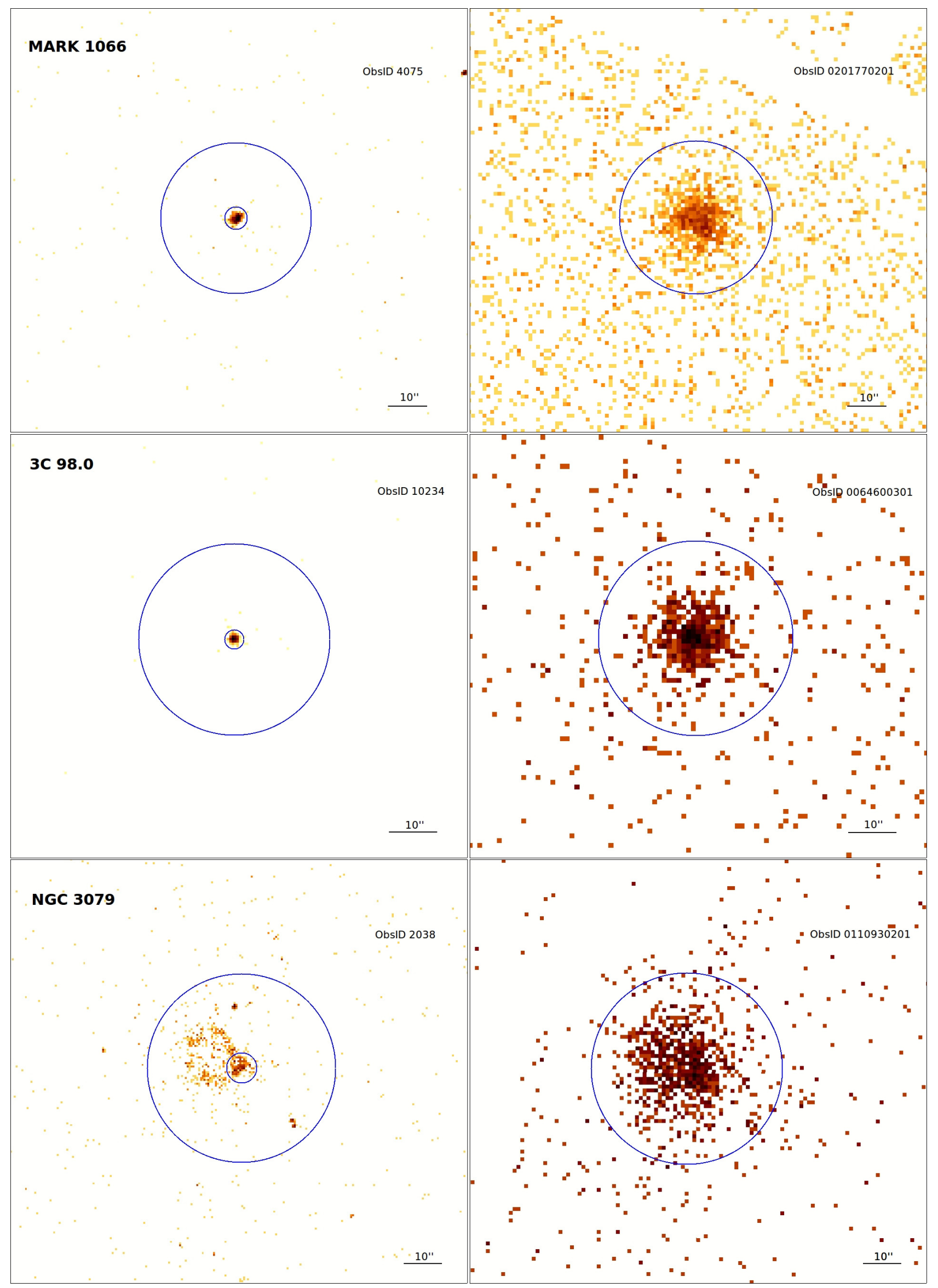

Fig. C.27. continued. 
A\&A 579, A90 (2015)

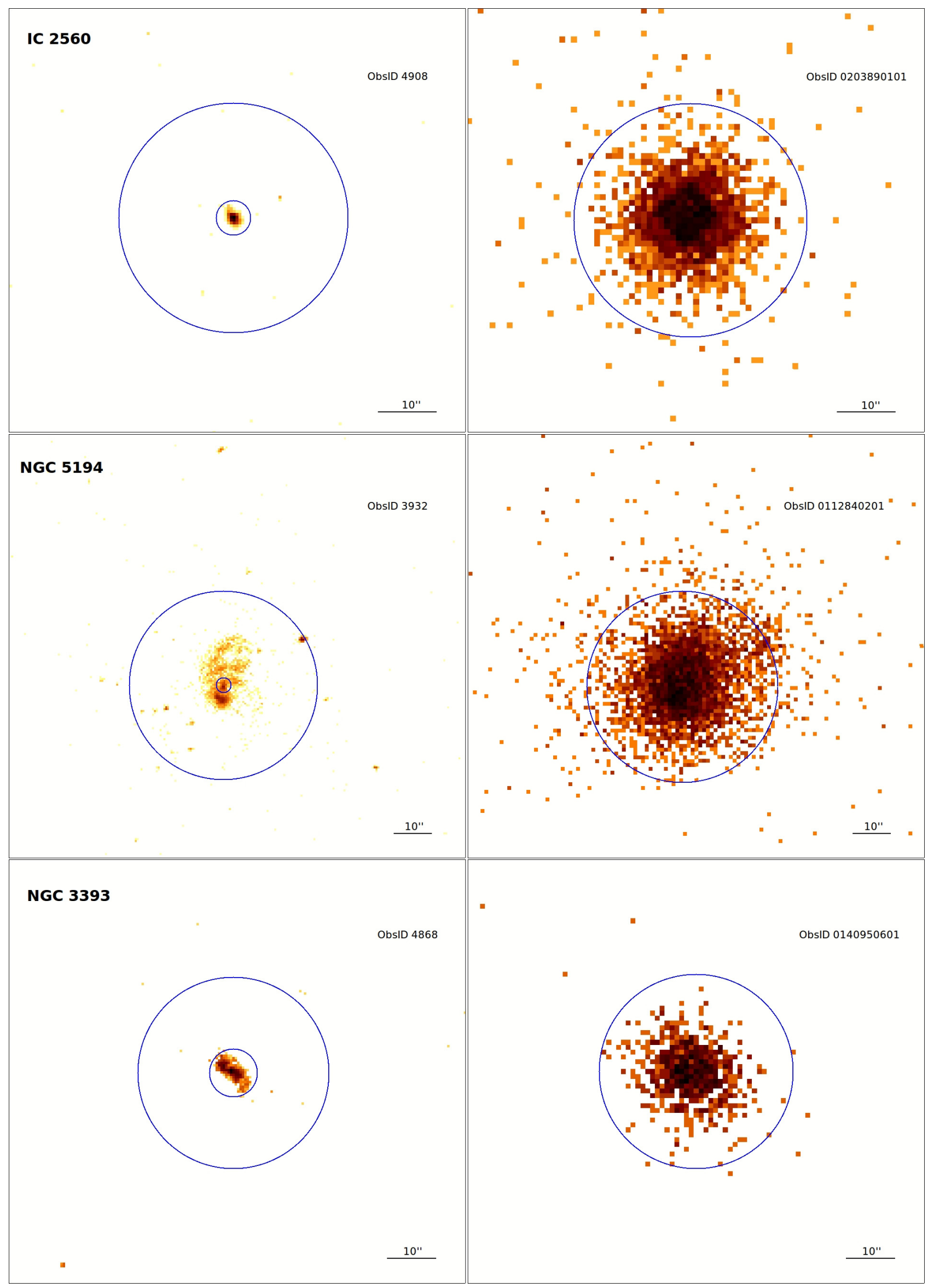

Fig. C.27. continued. 
L. Hernández-García et al.: X-ray variability of Seyfert 2s

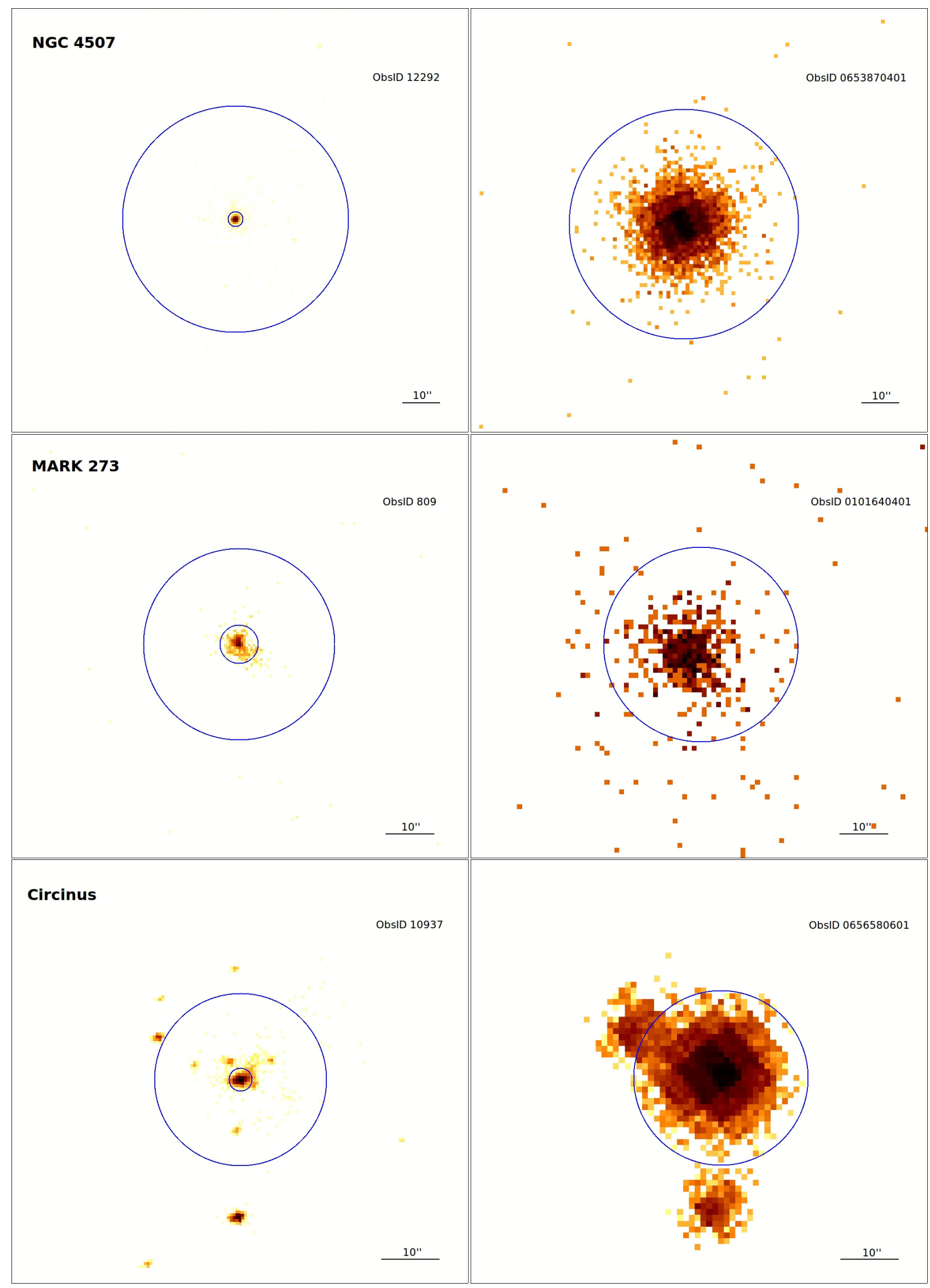

Fig. C.27. continued. 
A\&A 579, A90 (2015)

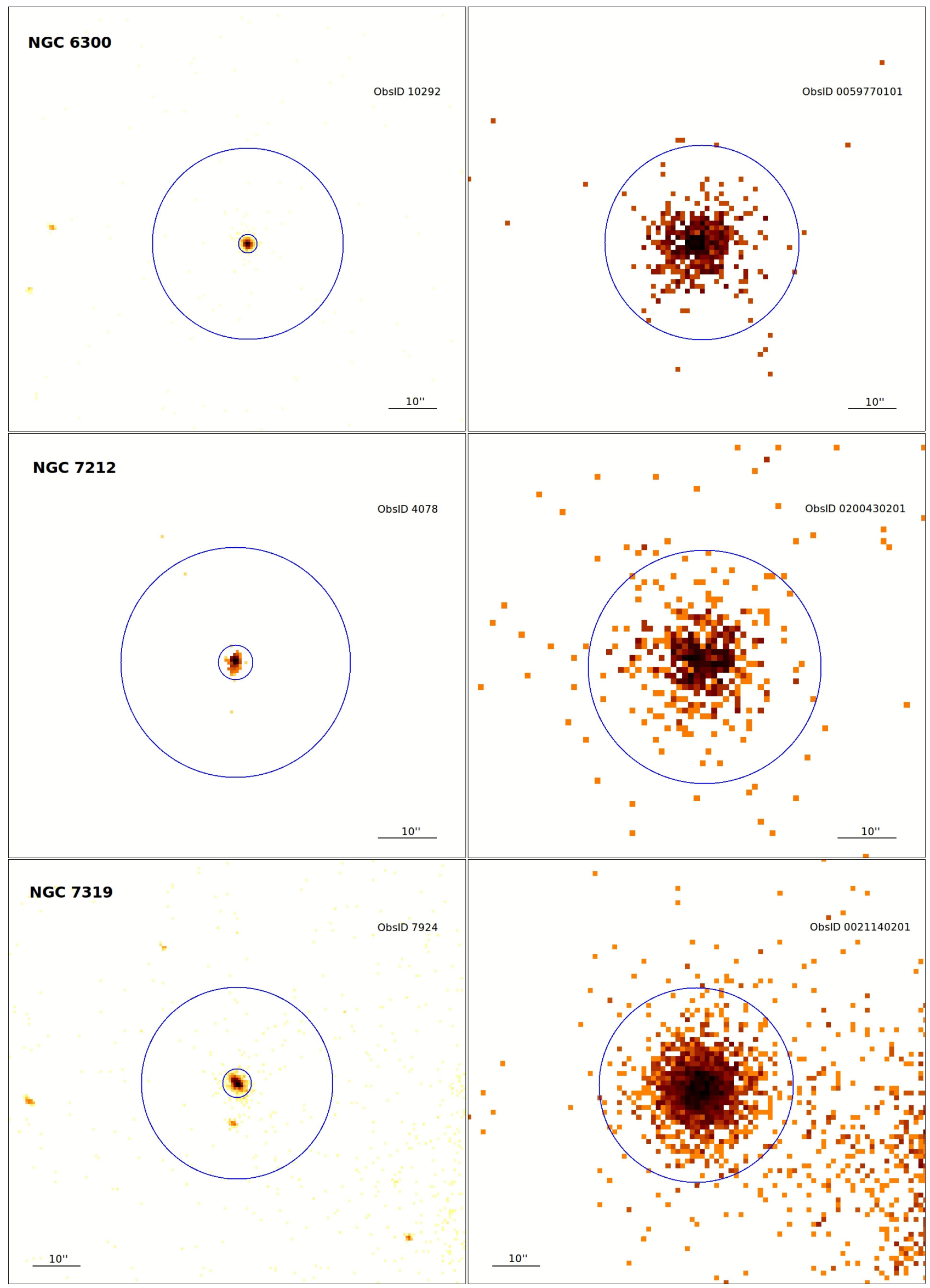

Fig. C.27. continued.

A90, page 68 of 77 


\section{Appendix D: Light curves}

This appendix provides the plots corresponding to the light curves. Three plots per observation are presented, corresponding to soft (left), hard (middle), and total (right) energy bands. Each light curve has a minimum of $30 \mathrm{ks}$ (i.e., 8 h) exposure time, while long light curves are divided into segments of $40 \mathrm{ks}$ (i.e., $11 \mathrm{~h}$ ). Each segment is enumerated in the title of the light curve. Count rates versus time continua are represented. The solid line represents the mean value, dashed lines the ${ }_{-}^{+} 1 \sigma$ from the average.
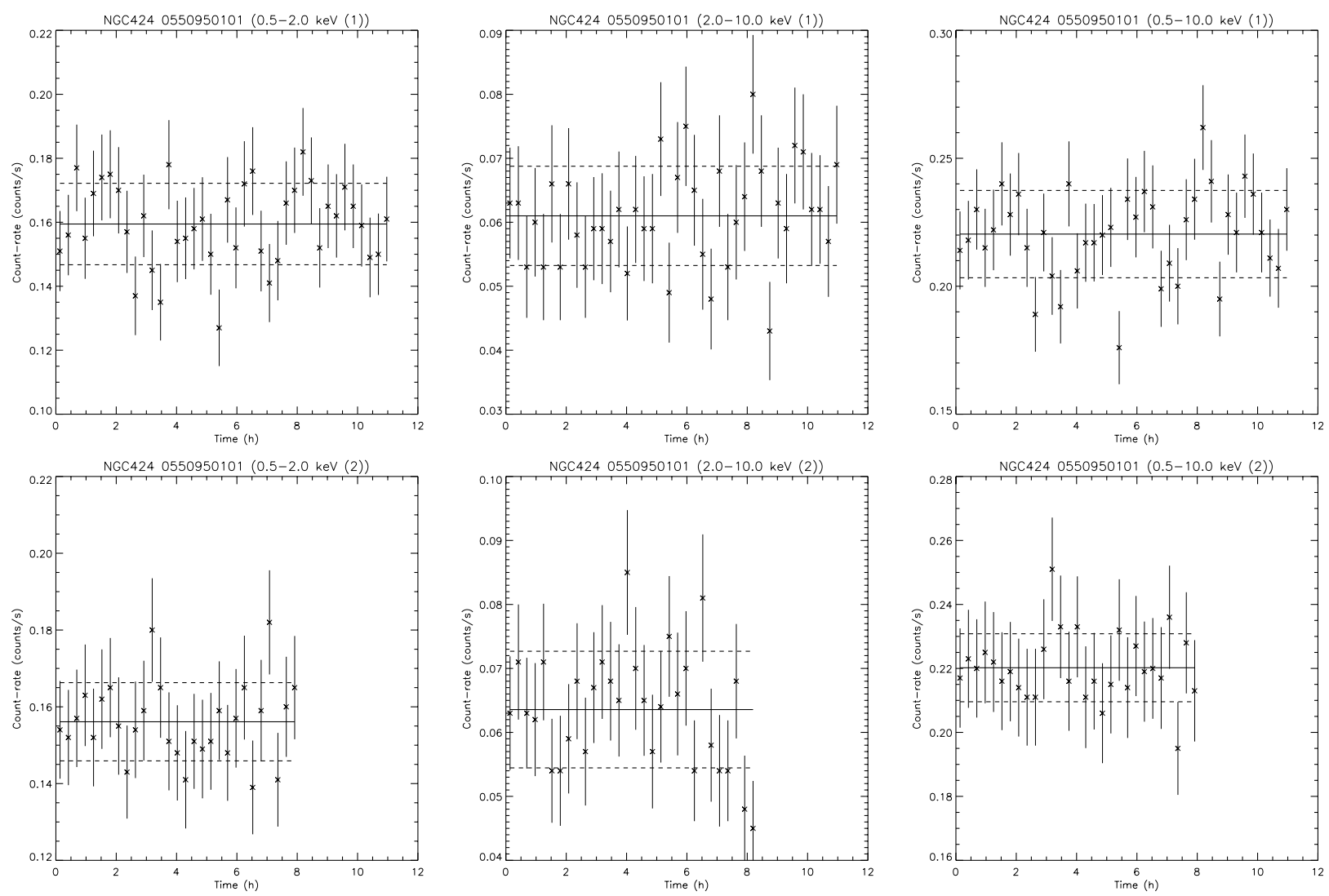

Fig. D.1. Light curves of NGC 424 from XMM-Newton data. 
A\&A 579, A90 (2015)
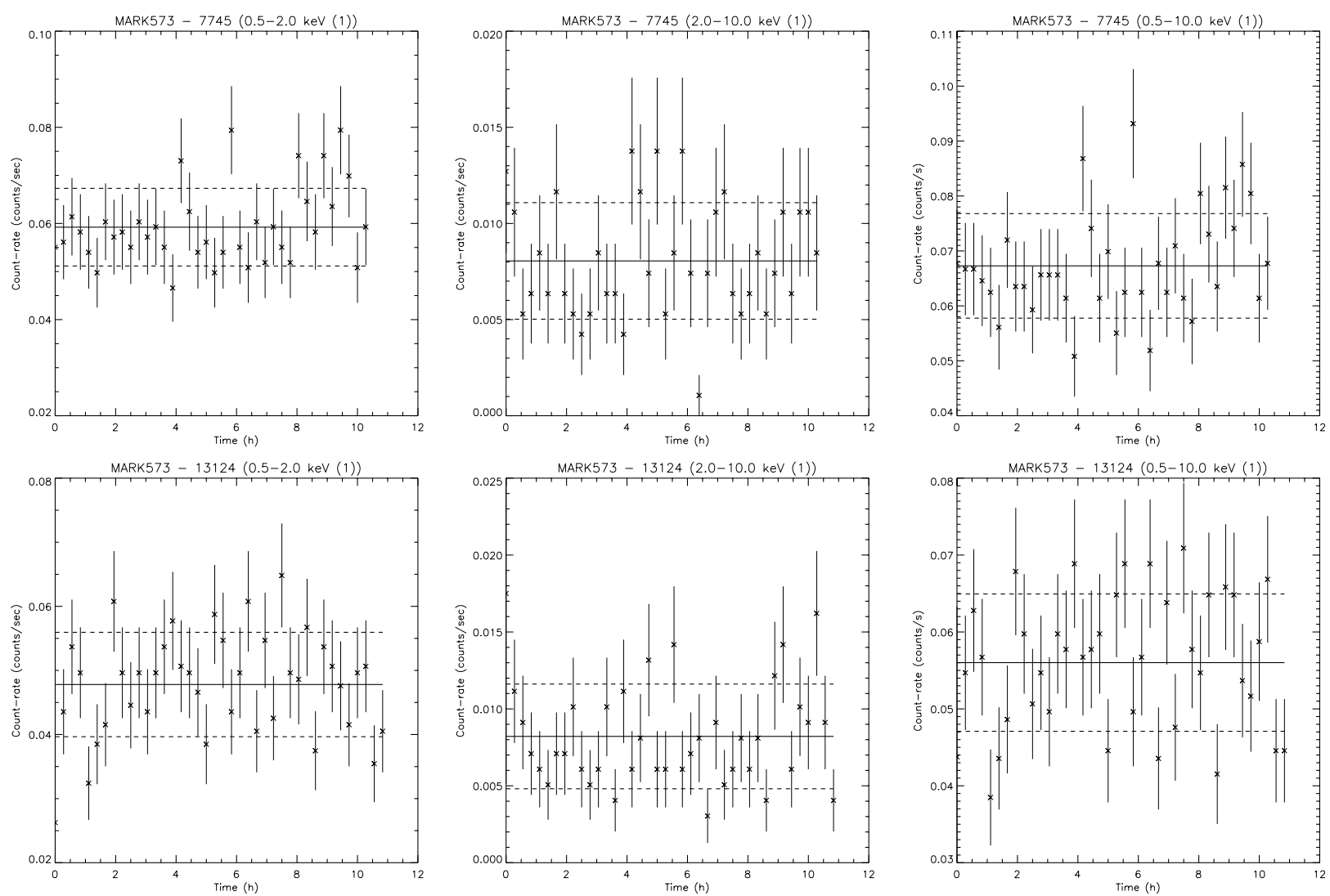

Fig. D.2. Light curves of MARK 573 from Chandra data.
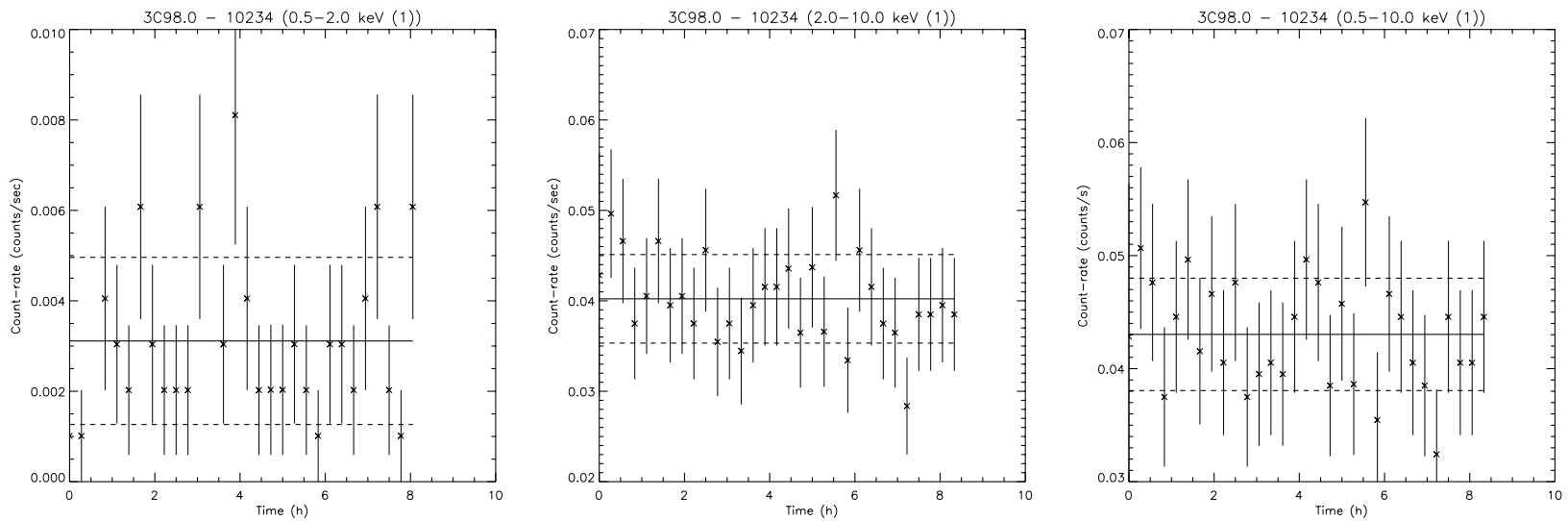

Fig. D.3. Light curves of 3C 98.0 from Chandra data. 
L. Hernández-García et al.: X-ray variability of Seyfert $2 \mathrm{~s}$
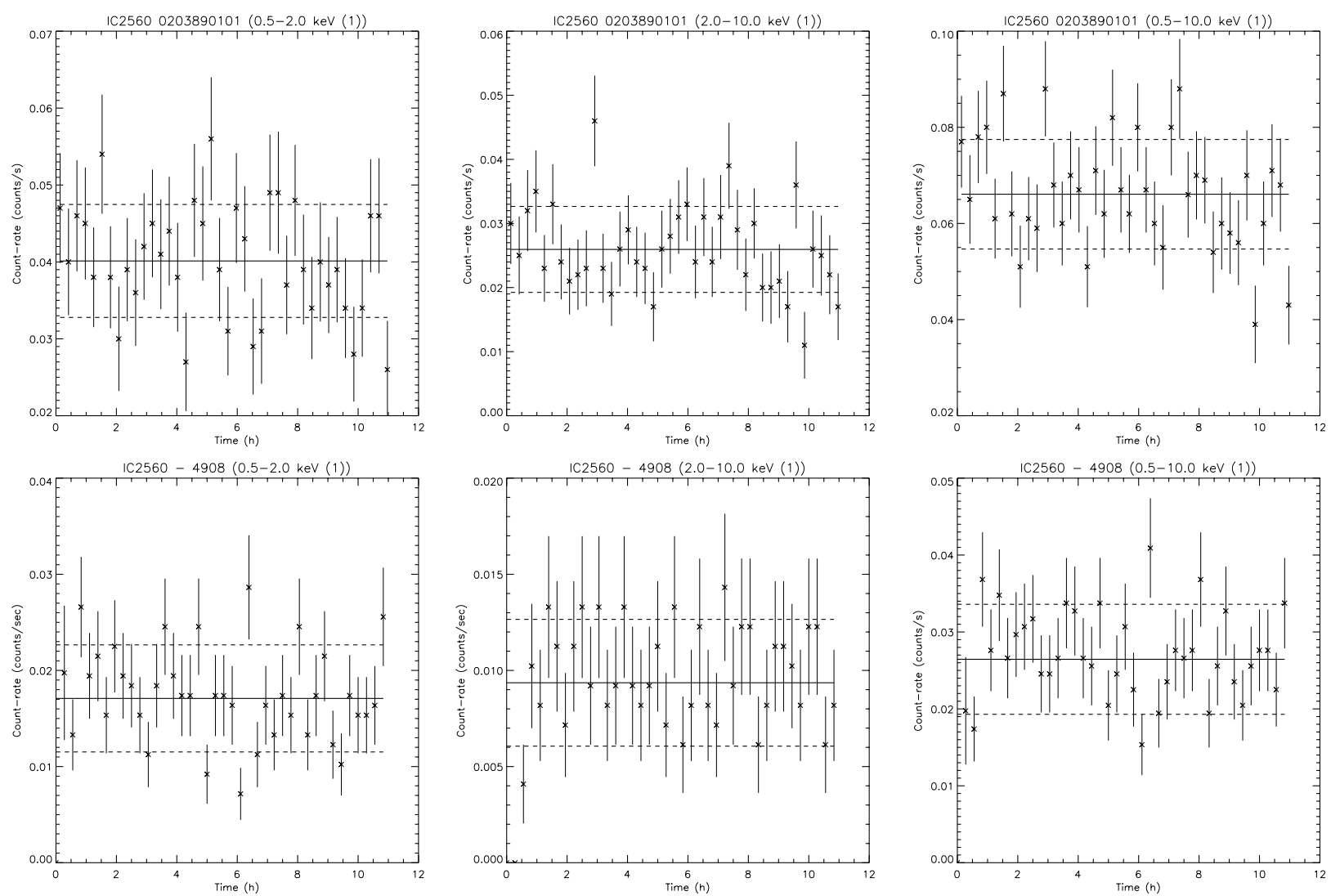

Fig. D.4. Light curves of IC 2560 from XMM-Newton and Chandra data.
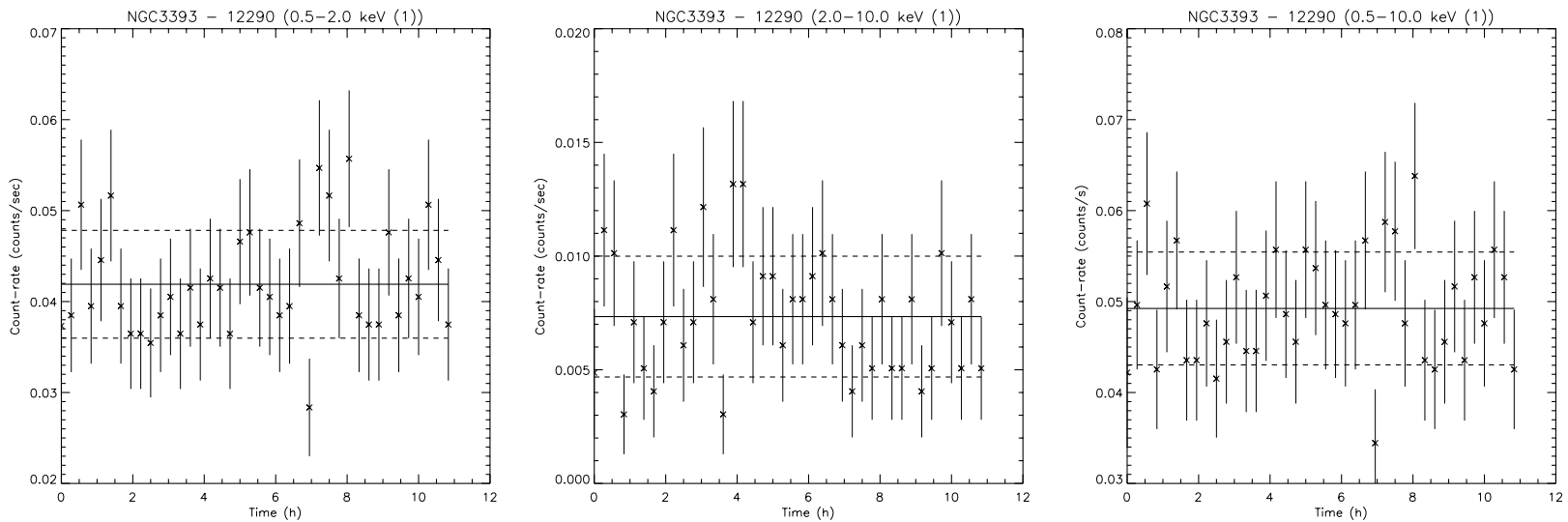

Fig. D.5. Light curves of NGC 3393 from Chandra data. 
A\&A 579, A90 (2015)
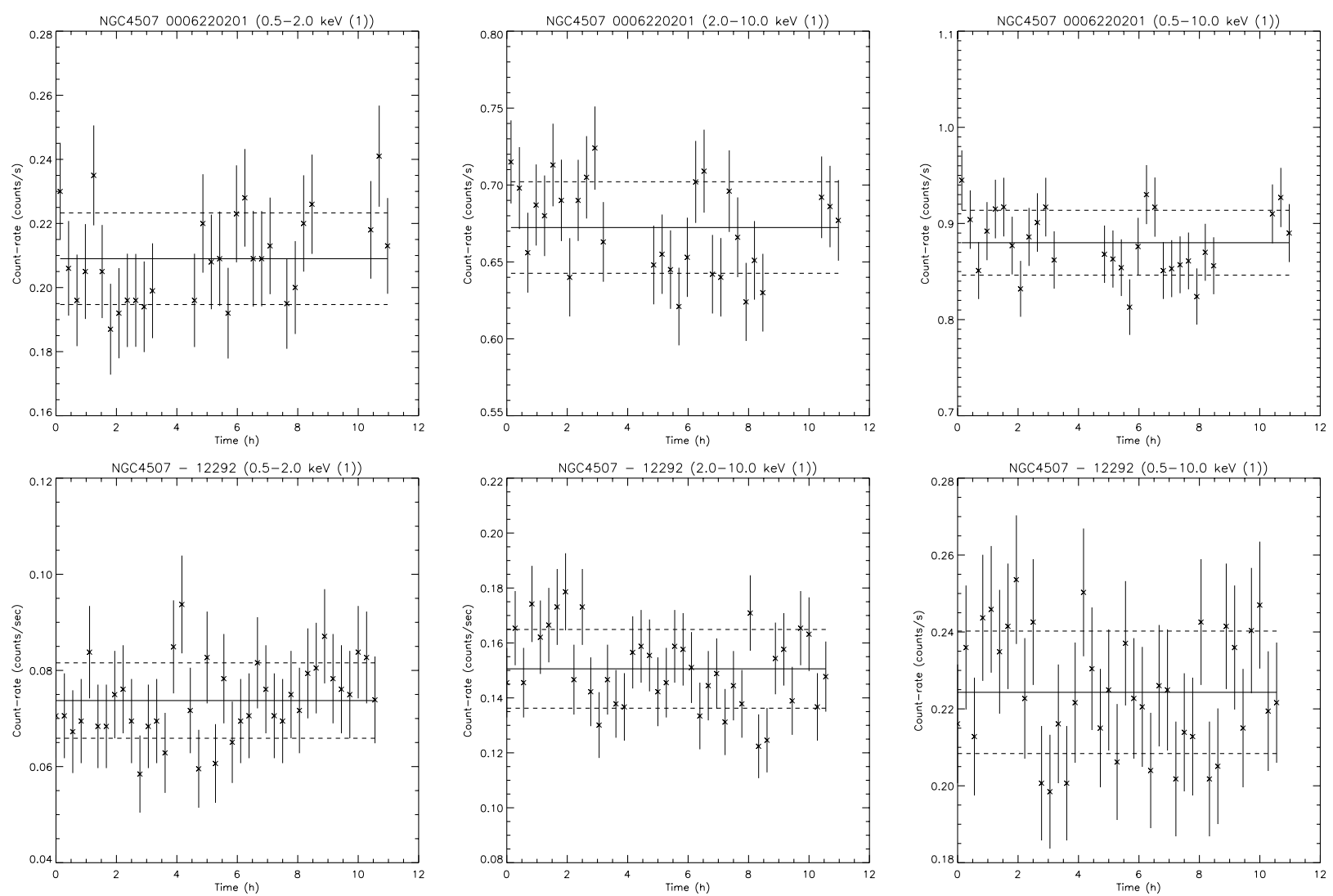

Fig. D.6. Light curves of NGC 4507 from XMM-Newton and Chandra data. 
L. Hernández-García et al.: X-ray variability of Seyfert 2s
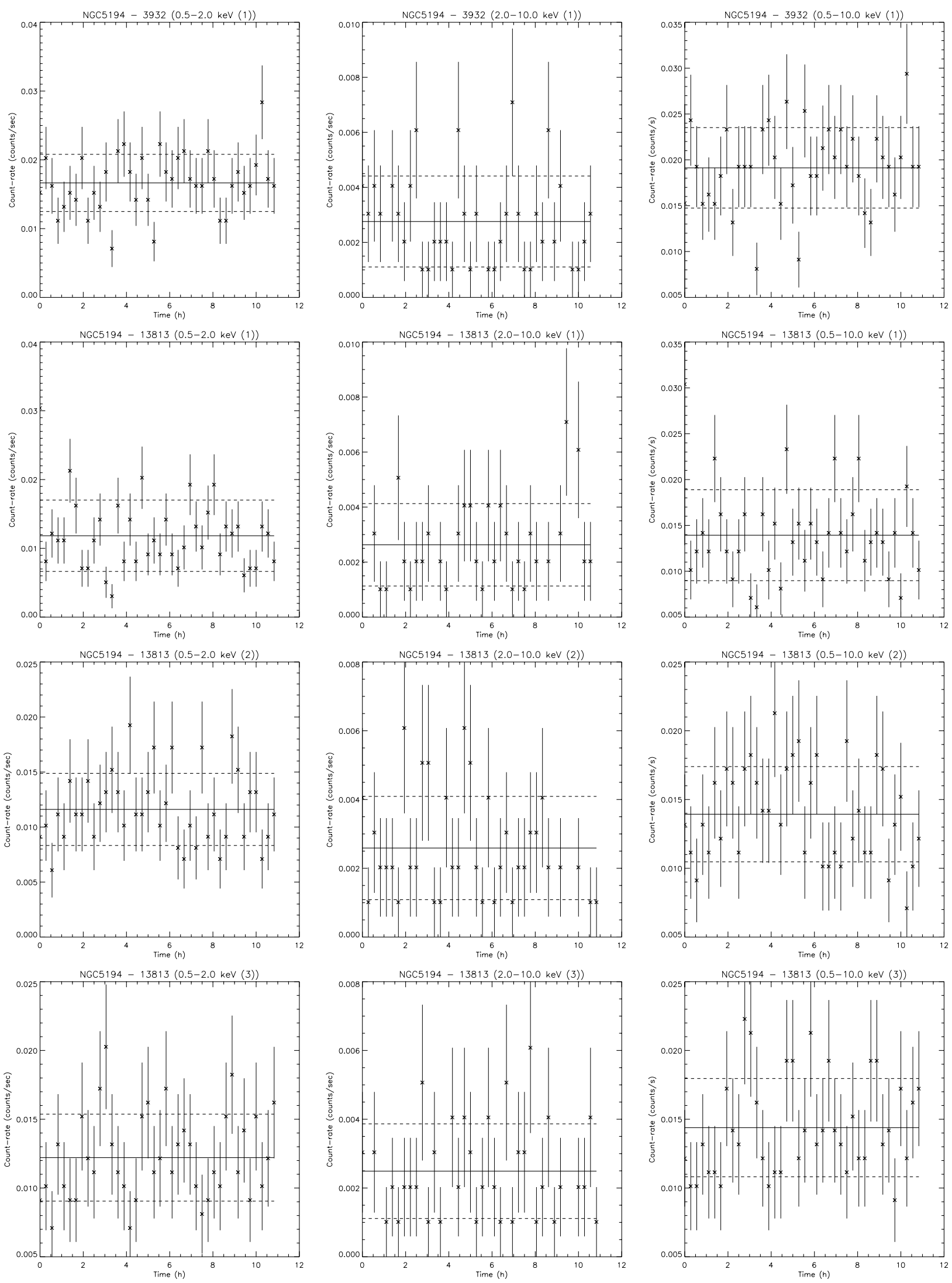

Fig. D.7. Light curves of NGC 5194 from Chandra data. 
A\&A 579, A90 (2015)
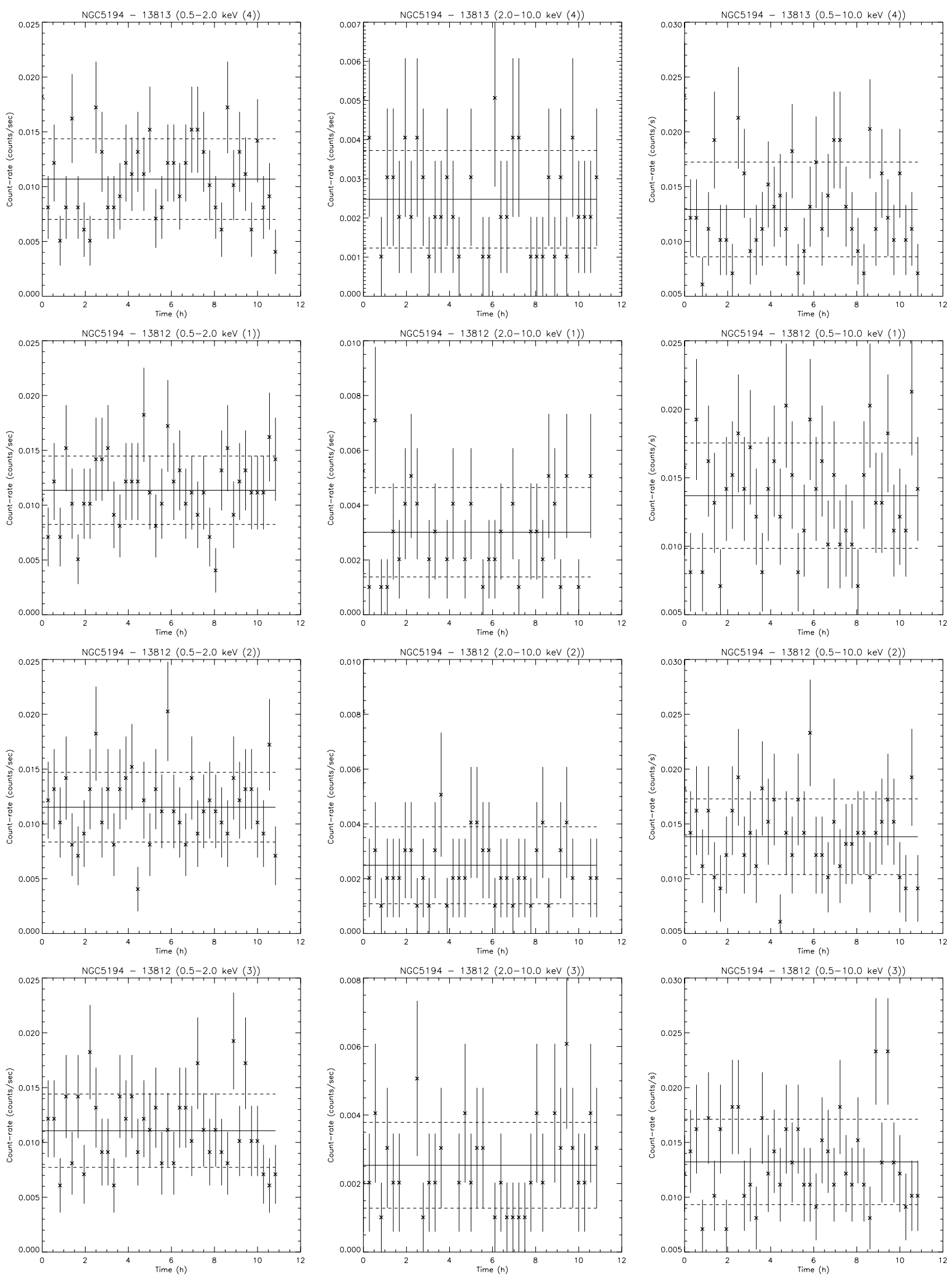

Fig. D.7. continued. 
L. Hernández-García et al.: X-ray variability of Seyfert 2s
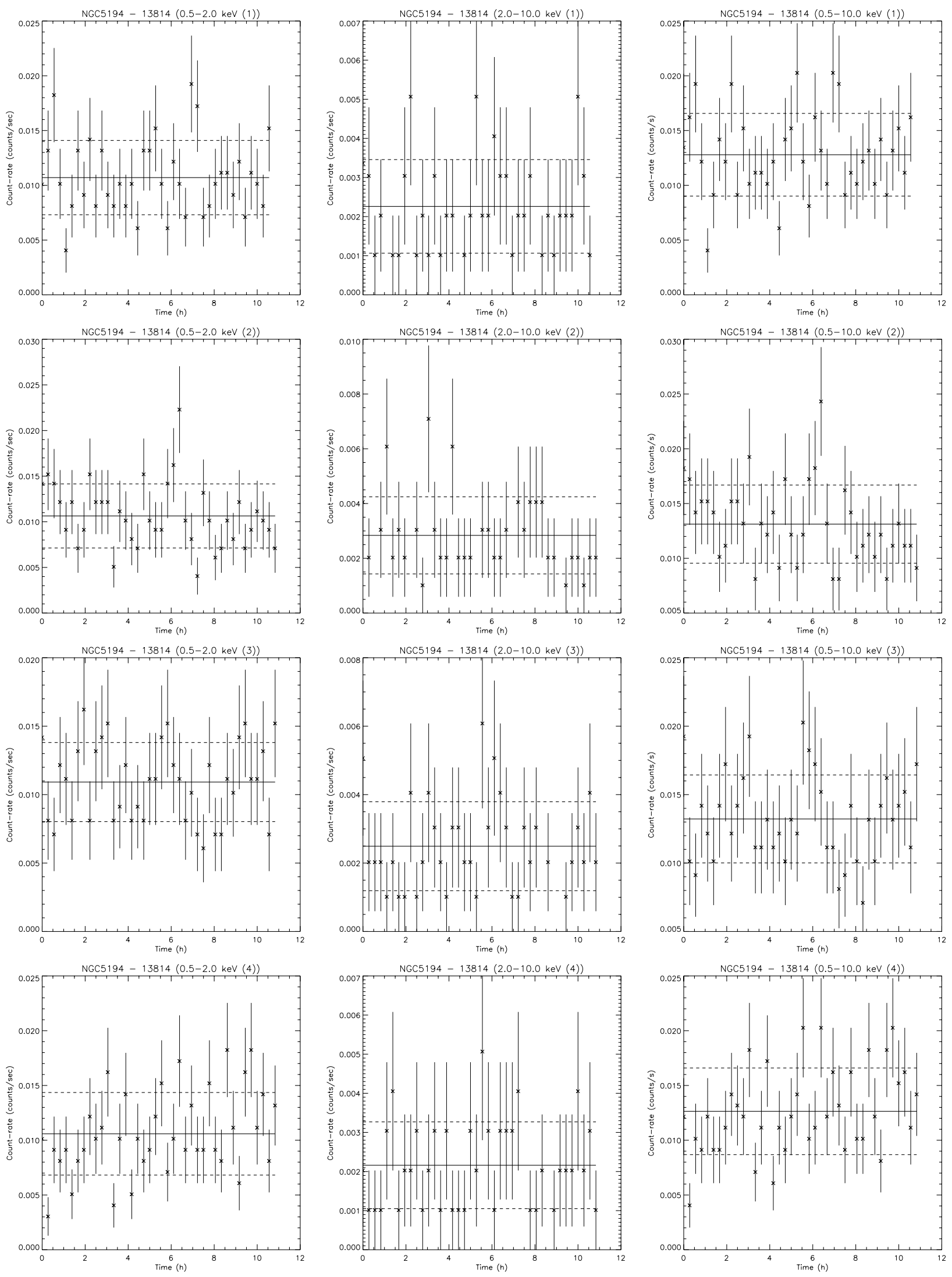

Fig. D.7. continued. 
A\&A 579, A90 (2015)
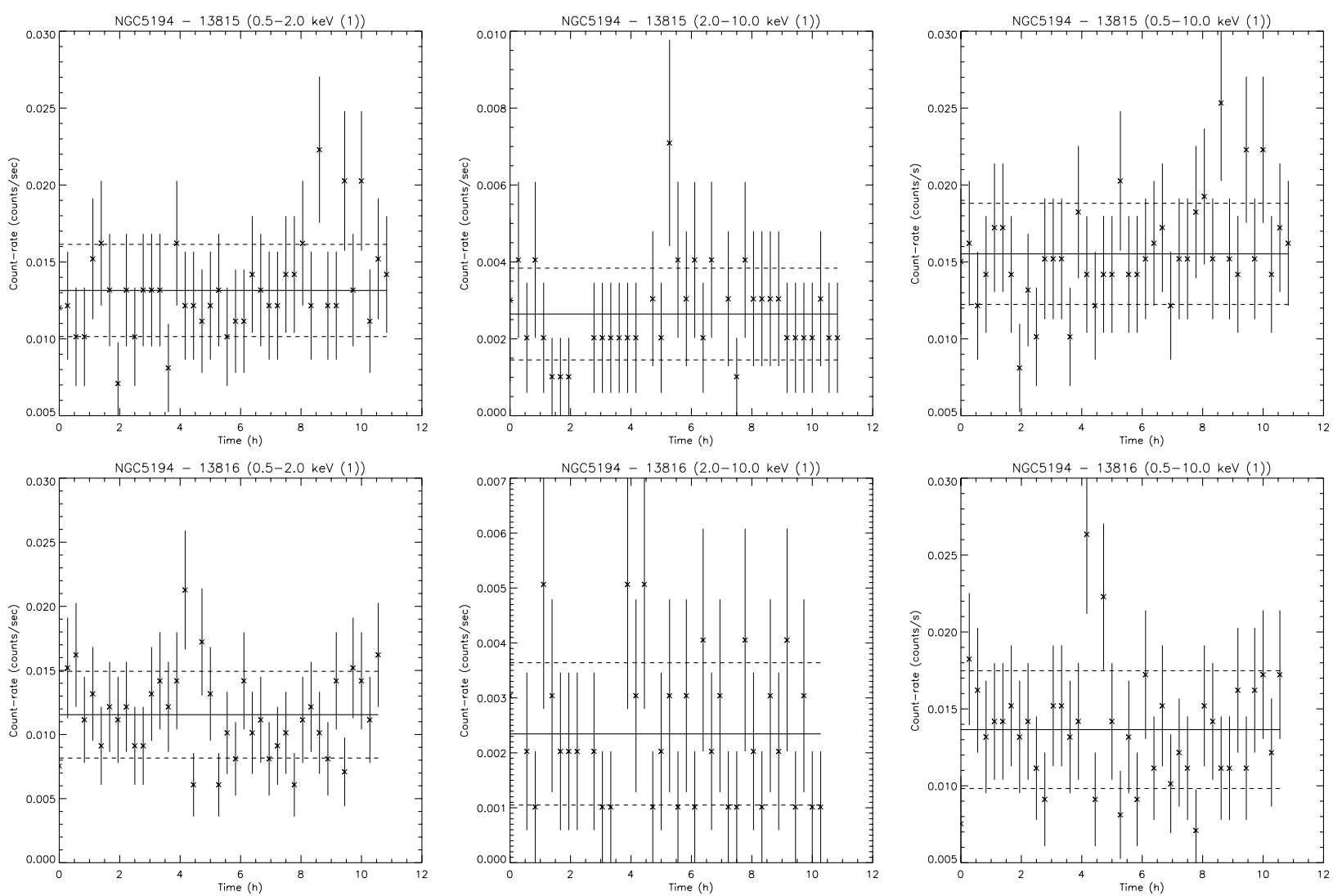

Fig. D.7. continued.
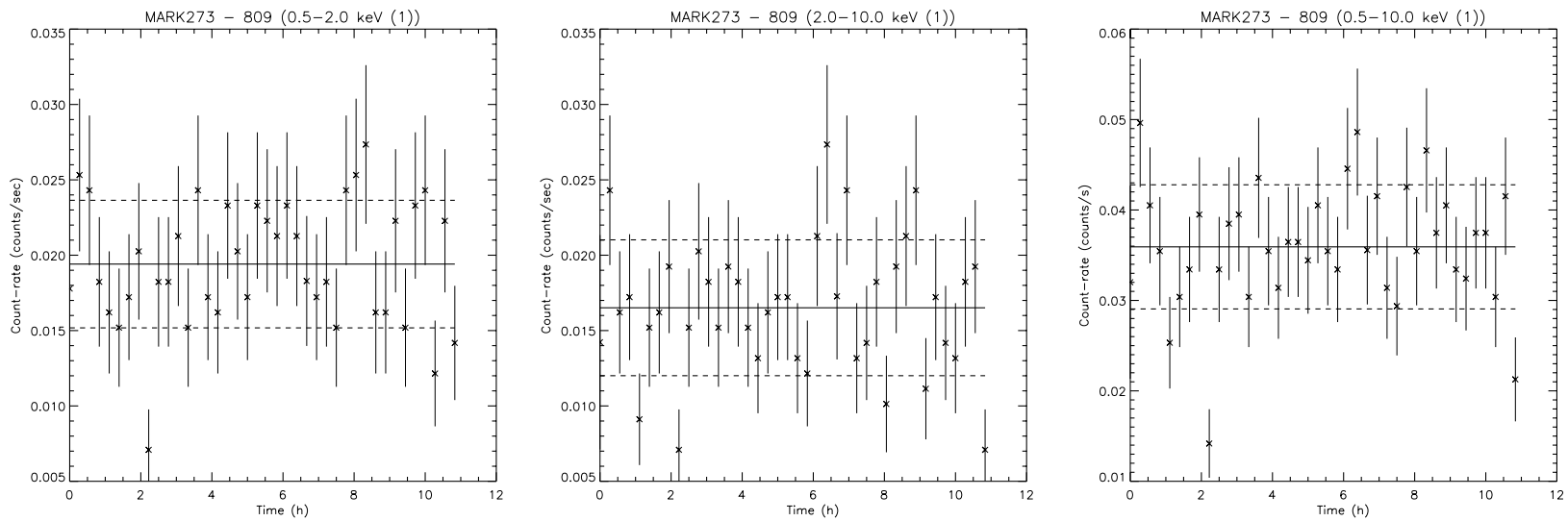

Fig. D.8. Light curves of MARK 273 from Chandra data.
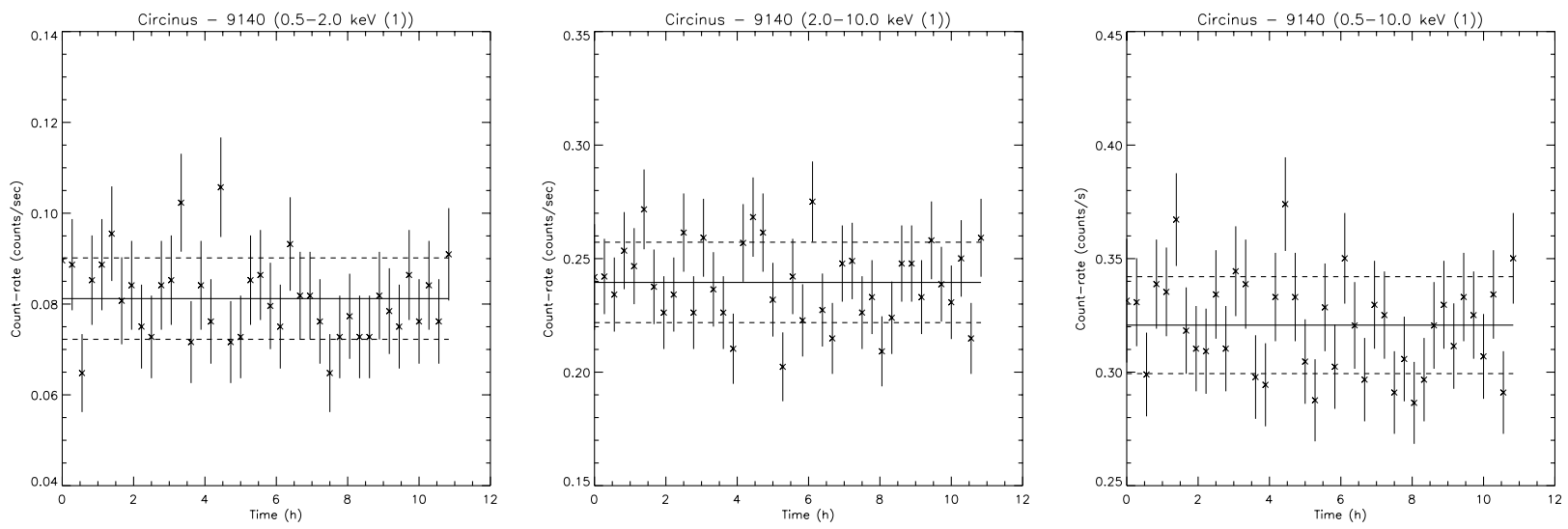

Fig. D.9. Light curves of Circinus from Chandra data. 
L. Hernández-García et al.: X-ray variability of Seyfert 2s
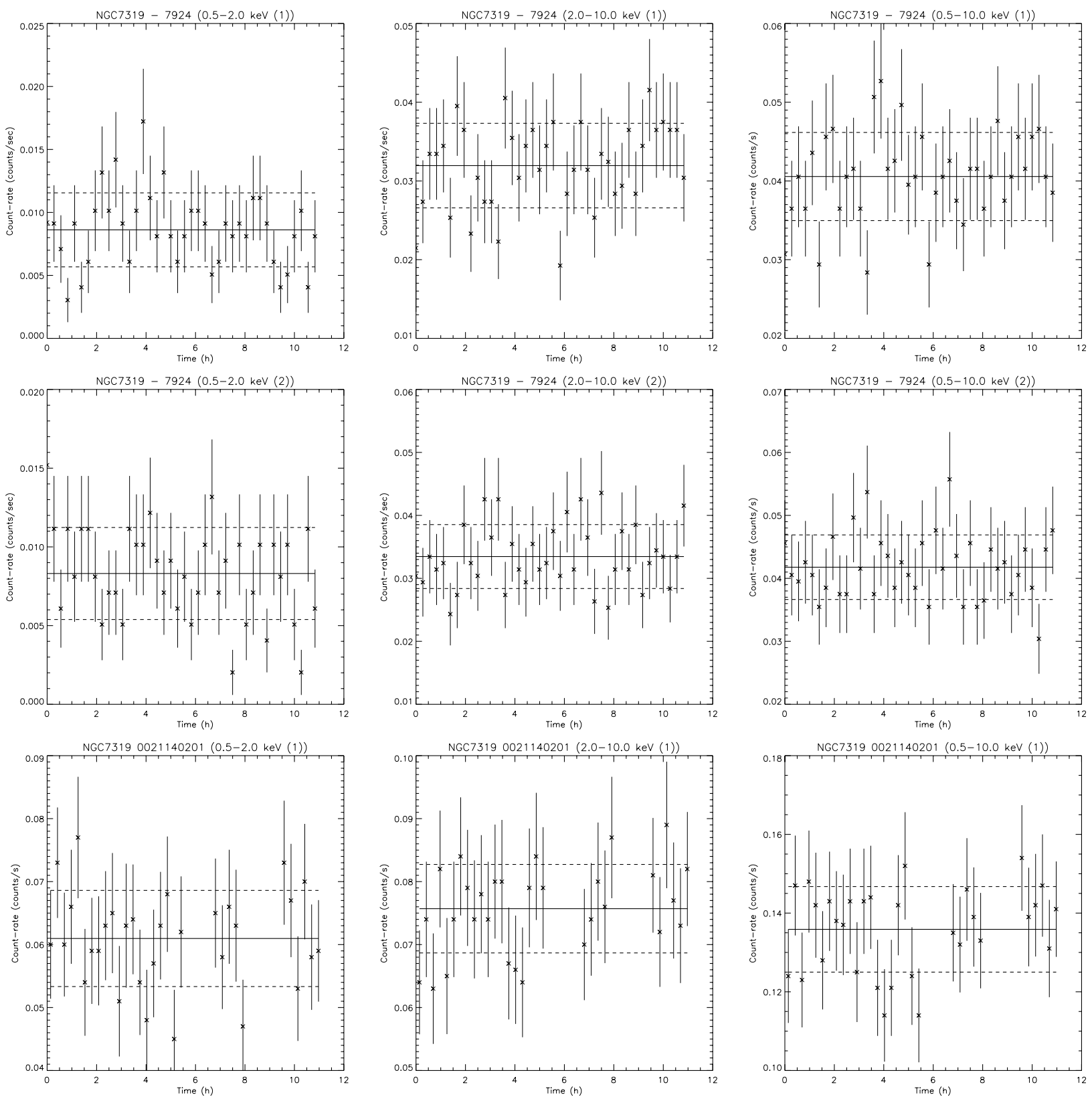

Fig. D.10. Light curves of NGC 7319 from Chandra and XMM-Newton data. 